University of Louisville

ThinkIR: The University of Louisville's Institutional Repository

Electronic Theses and Dissertations

$12-2017$

\title{
Metabolic regulation of myocardial adaptation to exercise.
}

Andrew Alan Gibb

University of Louisville

Follow this and additional works at: https://ir.library.louisville.edu/etd

Part of the Circulatory and Respiratory Physiology Commons

\section{Recommended Citation}

Gibb, Andrew Alan, "Metabolic regulation of myocardial adaptation to exercise." (2017). Electronic Theses and Dissertations. Paper 2878.

https://doi.org/10.18297/etd/2878

This Doctoral Dissertation is brought to you for free and open access by ThinkIR: The University of Louisville's Institutional Repository. It has been accepted for inclusion in Electronic Theses and Dissertations by an authorized administrator of ThinkIR: The University of Louisville's Institutional Repository. This title appears here courtesy of the author, who has retained all other copyrights. For more information, please contact thinkir@louisville.edu. 


\title{
METABOLIC REGULATION OF MYOCARDIAL ADAPTATION TO EXERCISE
}

\author{
By \\ Andrew Alan Gibb \\ B.S., Ohio University, 2009 \\ M.S., University of Louisville, 2011 \\ M.S., University of Louisville, 2015
}

\begin{abstract}
A Dissertation
Submitted to the Faculty of the University of Louisville School of Medicine in Partial Fulfillment of the Requirements for the Degree of
\end{abstract}

\author{
Doctor of Philosophy \\ In Physiology and Biophysics \\ Department of Physiology \\ University of Louisville, \\ Louisville, Kentucky
}

December 2017 



\title{
METABOLIC REGULATION OF MYOCARDIAL ADAPTATION TO EXERCISE
}

\author{
By \\ Andrew Alan Gibb \\ B.S., Ohio University, 2009 \\ M.S., University of Louisville, 2011 \\ M.S., University of Louisville, 2015
}

A Dissertation Approved on

September 1, 2017

By the following Dissertation Committee

Aruni Bhatnagar, Ph.D.

Bradford G. Hill, Ph.D.

Irving Joshua, Ph.D.

Dale Schuschke, Ph.D.

Claudio Maldonado, Ph.D. 


\section{ACKNOWLEDGEMENTS}

The completion of my graduate studies and the achievements thus far would not have been possible without the contributions of the many. Most importantly, I want to thank my wife, Jessica, for her unwavering love, encouragement, and understanding throughout this journey. With her, I know that all things are possible. I want to thank my parents, Kristen and Eric, for always inspiring me to pursue my dreams and providing me the opportunities to do so. To my younger siblings, Amanda and Cody, your continual support is something I deeply cherish. I extend my deepest thanks to my mentors, Drs. Bradford Hill and Aruni Bhatnagar, for believing in me, even when I did not. The curiosity, creativity, and integrity that define their scientific careers will serve as the foundation to develop mine. In addition to my mentors, I would like to acknowledge my committee members. Their input and guidance towards my education has been greatly appreciated. I would also like to thank Dr. Steve Jones, for teaching me the principles of cardiac physiology and importance rigor. To the members of the lab, both past and present, your contributions to my work have been invaluable and appreciated. It has been an amazing experience to learn from and work with you

all. I look forward to our continued friendships and collaborations in the years to come. Lastly, I would like to thank the American Heart Association, for providing me the fellowship to carry out my work. 


\section{ABSTRACT \\ METABOLIC REGULATION OF MYOCARDIAL \\ ADAPTATION TO EXERCISE}

Andrew Alan Gibb

September 1, 2017

While the benefits of exercise affect several organs, a significant adaptive response occurs within the heart. Exercise promotes cardiac growth, increases angiogenesis, and enhances cardiac function and these adaptations are associated with a cardioprotective phenotype. Additionally, extensive evidence shows that exercise dynamically regulates myocardial metabolism. This can be attributed to both changes in hormonal stimulation, increases in bioenergetic demand, and the bioavailability of circulating substrates. However, it is unclear whether these changes in metabolism contribute to physiologic cardiac growth. We reason that exercise-induced changes in metabolism are required to balance the catabolic and anabolic reactions needed for growth. Specifically, we hypothesize that exercise-induced changes in myocardial glycolytic activity are essential for activation of the physiological growth program and the synthesis of metabolites required for growth. Prior to investigating the effects of exercise on cardiac metabolism, we established a model of cardiac adaptation to exercise training in mice (Chapter II). Once established, as discussed in Chapter III, we found that the exercise-adapted heart demonstrates enhanced glycolytic activity; however, 
during exercise glycolytic rate in the heart is diminished. To determine whether high or low glycolytic rates may be a stimulus for cardiac growth, we assessed the effect of cardiac-specific overexpression of phosphofructokinase isoforms that decrease or increase myocardial glycolytic rate. We found that constitutive reduction in glycolytic rate was sufficient to structurally and functionally phenocopy the exercise-adapted heart, replete with activation of the physiologic growth program; however, metabolic inflexibility invoked by constitutive changes in glycolysis results in mild mitochondrial dysfunction. To assess how glycolytic activity affects anabolic metabolism, we utilized stable isotope labeling in an in vitro model to interrogate flux of carbon into collateral biosynthetic pathways of glucose metabolism. Results from these studies, discussed in Chapter IV, show that PFK1 and glycolytic activity coordinate the allocation of glucose into pathways that would be important for cardiac growth. Collectively, our results indicate that physiological growth of the heart is intricately regulated by exercise-induced changes in intermediary metabolism, and that preservation of metabolic flexibility is essential for mitochondrial health. Importantly, these findings suggest a causal role of metabolism in myocardial responses to stress. 
GRAPHICAL ABSTRACT
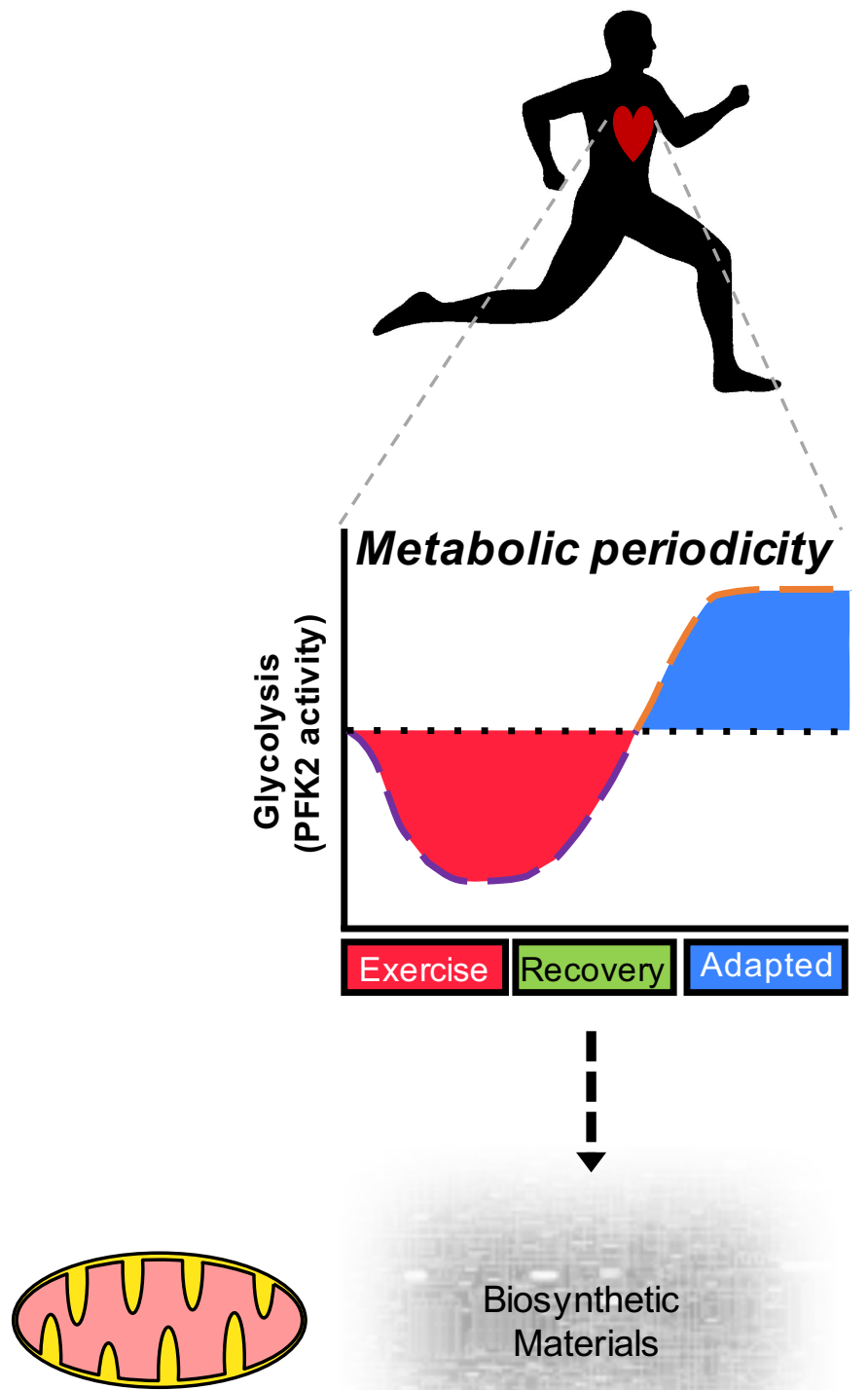

Biosynthetic

Materials

Adaptive Gene

Program

Healthy Mitochondria

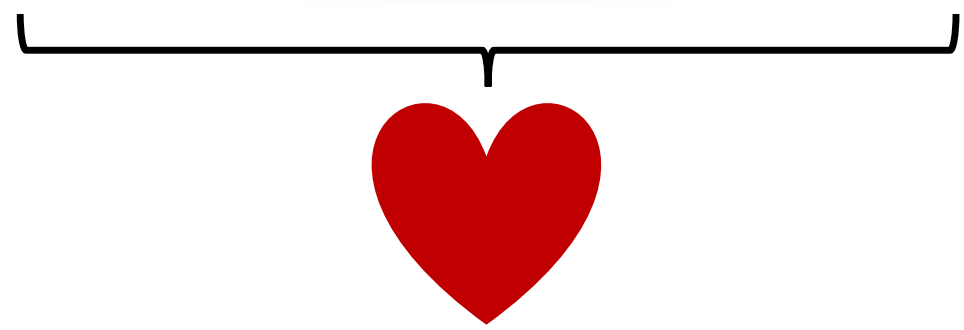

Physiologic

Cardiac Growth 


\section{TABLE OF CONTENTS}

PAGE

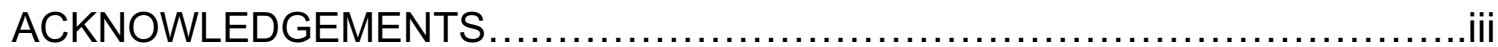

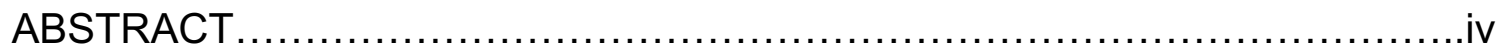

GRAPHICAL ABSTRACT ........................................................

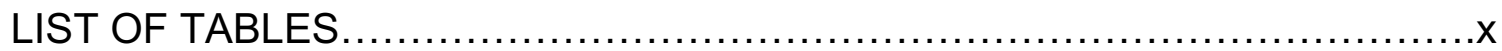

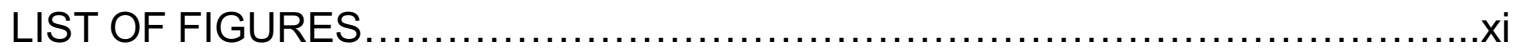

CHAPTER I

GENERAL INTRODUCTION

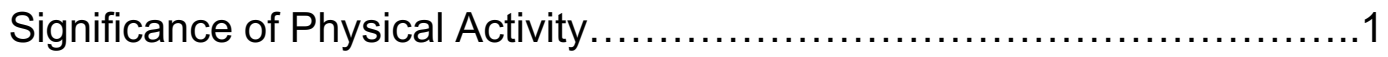

Exercise and the Cardiovascular System.................................

Cardiac Metabolism in Exercise.........................................18

Metabolism as a Contributing Factor to Growth..........................29

CHAPTER II

FVB/NJ MICE ARE A USEFUL MODEL FOR EXAMINING CARDIAC ADAPTATIONS TO TREADMILL TRAINING 
Introduction.

Experimental Procedure................................................. 35

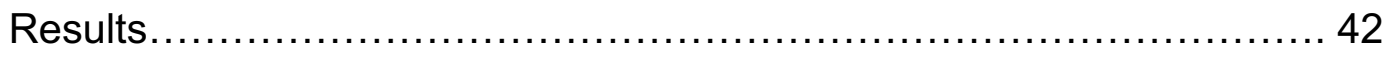

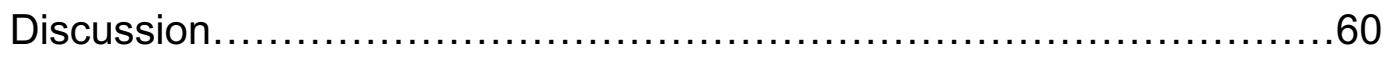

\section{CHAPTER III}

EXERCISE-INDUCED CHANGES IN GLUCOSE METABOLISM PROMOTE PHYSIOLOGIC CARDIAC GROWTH

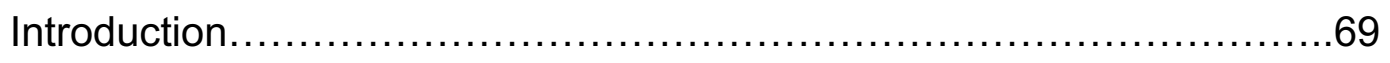

Experimental Procedure .................................................. 71

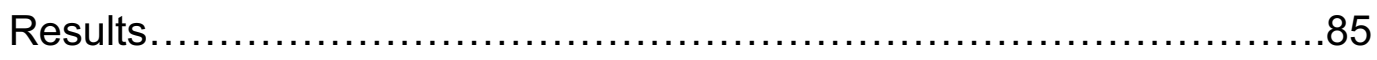

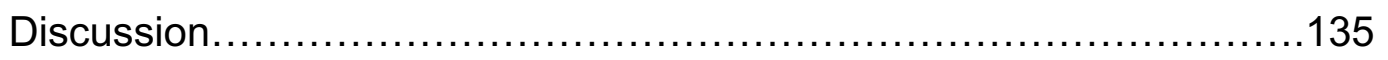

\section{CHAPTER IV}

INTEGRATION OF FLUX MEASUREMENTS TO RESOLVE CHANGES IN ANABOLIC AND CATABOLIC METABOLISM IN CARDIAC MYOCYTES

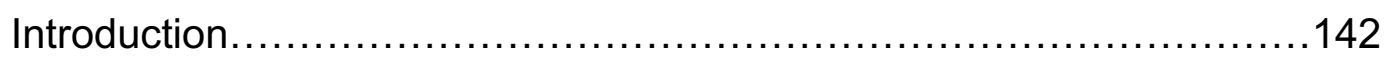

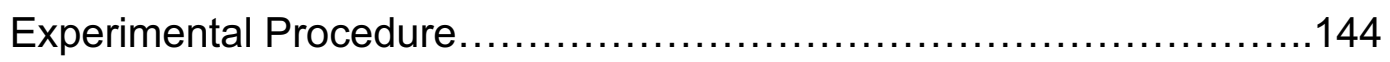

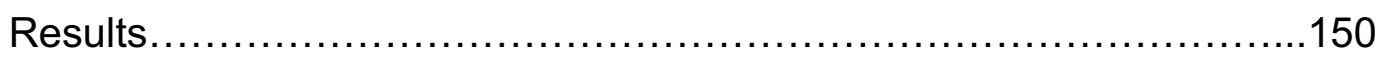

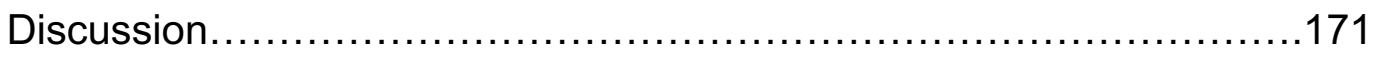


CHAPTER V

CONCLUDING DISCUSSION..........................................179

Metabolic Regulation of Cardiac Growth............................... 182

Future Directions .....................................................

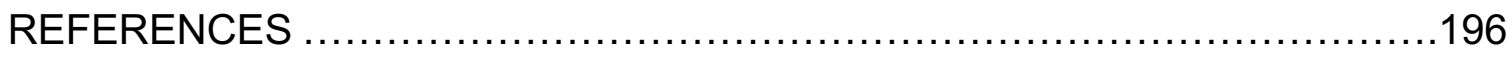

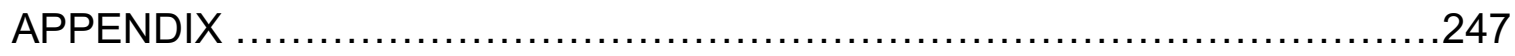

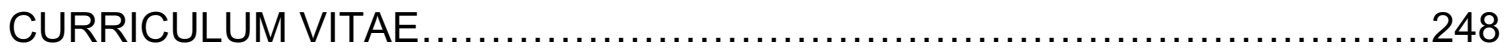




\section{LIST OF TABLES}

TABLE

PAGE

1. Exercise compliance chart................................................. 52

2. Measurements of exercise-induced cardiac growth......................... 58

3. Echocardiographic data from sedentary and exercised WT mice..............90

4. Significantly different metabolites in hearts from exercise-adapted mice.......95

5. Significantly different metabolites in hearts from WT and Glyco ${ }^{\mathrm{LO}}$ mice $\ldots . .110$

6. Significantly different metabolites in hearts from WT and Glyco ${ }^{\mathrm{Hi}}$ mice $\ldots \ldots . .114$

7. Echocardiographic parameters from Glyco ${ }^{\mathrm{Lo}}$ and $\mathrm{Glyco}^{\mathrm{Hi}}$ mice compared with

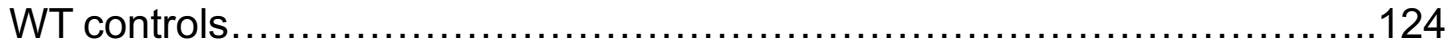




\section{LIST OF FIGURES}

FIGURE

PAGE

1. Summary of the cardiac benefits of exercise ................................... 4

2. Exercise-mediated cardiac adaptive signaling pathways.....................12

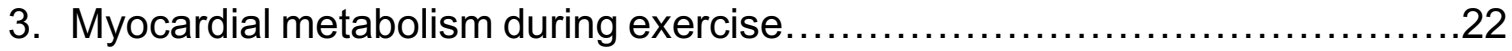

4. Exercise testing and training design .........................................

5. Chronobiological characteristics of C57BL/6J and FVB/NJ mice...............44

6. FVB/NJ mice display a higher initial exercise capacity than C57BL/6J mice ........................................................................ 47

7. Compliance of FVB/NJ and C57BL/6J to the treadmill training................50

8. Treadmill training-induced improvements in exercise capacity in

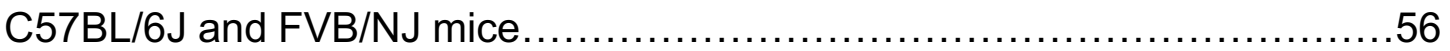

9. FVB/NJ mice display robust cardiometabolic adaptations to treadmill exercise

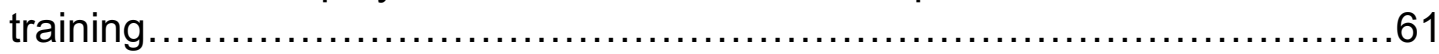

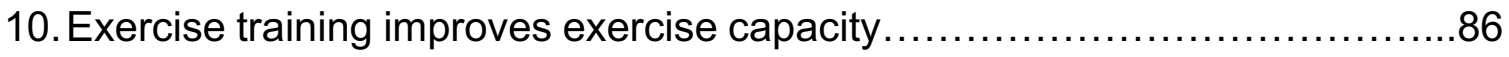

11. Exercise training promotes physiologic cardiac growth......................88

12. Metabolomic changes in the exercise-adapted mouse heart..................93

13. Exercise dynamically regulates PFK2 and glycolysis in the heart..............99

14. Exercise training does not alter the abundance of key glucose-metabolizing enzymes.

15. Cardiac F-2,6- $\mathrm{P}_{2}$ regulates myocardial glucose and lipid metabolism........106

16. Metabolomic changes in the $\mathrm{Glyco}^{\mathrm{Lo}}$ and $\mathrm{Glyco}^{\mathrm{Hi}}$ mouse hearts...............108 
17. Modulation of cardiac glycolytic rate regulates lipid, carbohydrate, and amino acid metabolism

18. Constitutive changes in glycolysis promote cardiac growth and

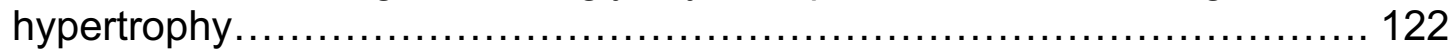

19. Metabolic inflexibility disrupts cristae structure and causes mitochondrial dysfunction 128

20. Low myocardial glycolytic rates are sufficient for maximal cardiac growth....130

21. Myocardial glycolysis coordinately regulates gene transcription in the heart.133

22. Phosphofructokinase activity regulates glucose metabolism in isolated cardiomyocytes.

23. Phosphofructokinase-mediated glucose distribution in isolated cardiomyocytes. 155

24. Phosphofructokinase coordinates pentose phosphate pathway

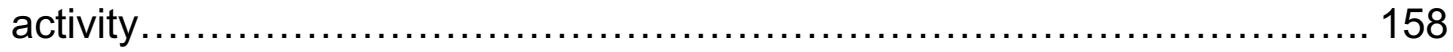

25. High phosphofructokinase activity diminishes glucose carbon incorporation into

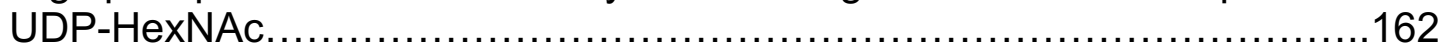

26. Phosphofructokinase regulates glycerolipid biosynthesis...................166

27. Phosphofructokinase activity regulates mitochondrial activity in isolated cardiomyocytes.

28. Exercise-induced effects on cardiac metabolism and its role in regulating cardiac growth and mitochondrial health. 183

29. Metabolic regulation of the biosynthetic pathways involved in the cardiac growth response. 187 


\section{CHAPTER I}

\section{GENERAL INTRODUCTION}

\section{Significance of Physical Activity}

Physical activity is firmly embedded in human culture. The ability to adapt to periods of increased physical activity likely played a critical role in the evolution of human physiology. For instance, our early ancestors with low "physical fitness," likely did not fare well at a time when hunting, gathering, shelter building, and evasion from predators were a necessity. This period in time likely contributed to the evolutionary physiologic advancement of the human species. Much later in ancient Greece, Hippocrates was the first to realize the importance of physical activity and stated that "eating alone will not keep a man well; he must also take exercise. For food and exercise...work together to produce health." ${ }^{1}$ Today, physical activity as a way of life is simply an afterthought. With the obvious transition from manual labor jobs to sedentary desk jobs and the technological advancements made over the last century, a sedentary lifestyle has become the new "normal" for human physiology. Unfortunately, this new "normal" could be considered evolutionarily "abnormal." While exercise has been labeled as providing "health benefits," it is in fact the lack of exercise that carries health risks. ${ }^{2}$ This deviation from "normal" and change in lifestyle led the World Health 
Organization (WHO) to classify physical inactivity as the fourth leading risk factor for global mortality. ${ }^{3}$

While physical inactivity is a health risk, it is a modifiable one. The first documented study of the beneficial effects of an active lifestyle was in 1953. This study showed that those in more physically active jobs (e.g., train conductors and postmen) in London had a lower incidence of cardiovascular events and a lower early mortality-rate than their sedentary, desk-based, civil servant counterparts. ${ }^{4}$ Along with this observation, the benefits of physical activity have only become more noticeable over the years. Regular exercise has been shown to promote cardiovascular health, ${ }^{5-7}$ augment skeletal muscle function, ${ }^{8}$ and increase both healthspan ${ }^{8-10}$ and lifespan. ${ }^{5,}$ 11-13 An individual's level of physical fitness, commonly measured by their exercise capacity, is a more powerful predictor of mortality among men than other established cardiovascular risk factors such as hypertension, blood lipids, and smoking. ${ }^{12}$ The indication that physical fitness is the most powerful predictor of mortality risk highlights the importance of an active lifestyle.

Despite irrefutable evidence that exercise imparts health benefits, or perhaps more accurately prevents sedentary health risks, the mechanisms by which exercise does so remain poorly understood. For instance, epidemiologic evidence suggests that while exercise training significantly lowers traditional cardiovascular risk factors (i.e. hypertension, inflammation, and blood lipids), only half of the reduction in event risk can be explained by these changes. ${ }^{6,7}$ These 
observations suggest that a large portion of the benefits of exercise remain unexplained and warrant further investigation.

\section{Exercise and the Cardiovascular System}

The complexity of studying exercise is rooted in the coordinated response of the body's organ systems. For example, even before beginning exercise (e.g. running), the centers in the brain prime the body for an increase in energy expenditure by increasing respiratory and heart rates (refs). At the onset of exercise, skeletal muscle contracts in response to the increased workload, requiring an increase in the utilization of fuel substrates such as glucose, glycogen, and fatty acids to meet the higher energy demand. This increase in muscle metabolism must be met by an equal increase in oxygen delivery, which is accomplished by a further increase in pulmonary respiratory rates and heart rates to increase oxygen delivery. These changes from homeostasis in the muscular, respiratory and circulatory systems are driven in part by changes in both the autonomic tone of the nervous system and the release of circulating hormones and catecholamines from the endocrine system. Interestingly, exercise profoundly affects the cardiovascular system in particular, resulting in significant adaptive responses of the heart. For instance, exercise promotes cardiac growth, increases perfusion, and enhances cardiac function and substrate utilization, all of which contribute to cardioprotection (Fig. 1). Understanding the mechanisms that underlie this response could lead to therapeutic strategies to improve cardiac health in disease. 
Figure 1: Summary of the cardiac benefits of exercise. SV, stroke volume; CO, cardiac output. 
FIGURE 1

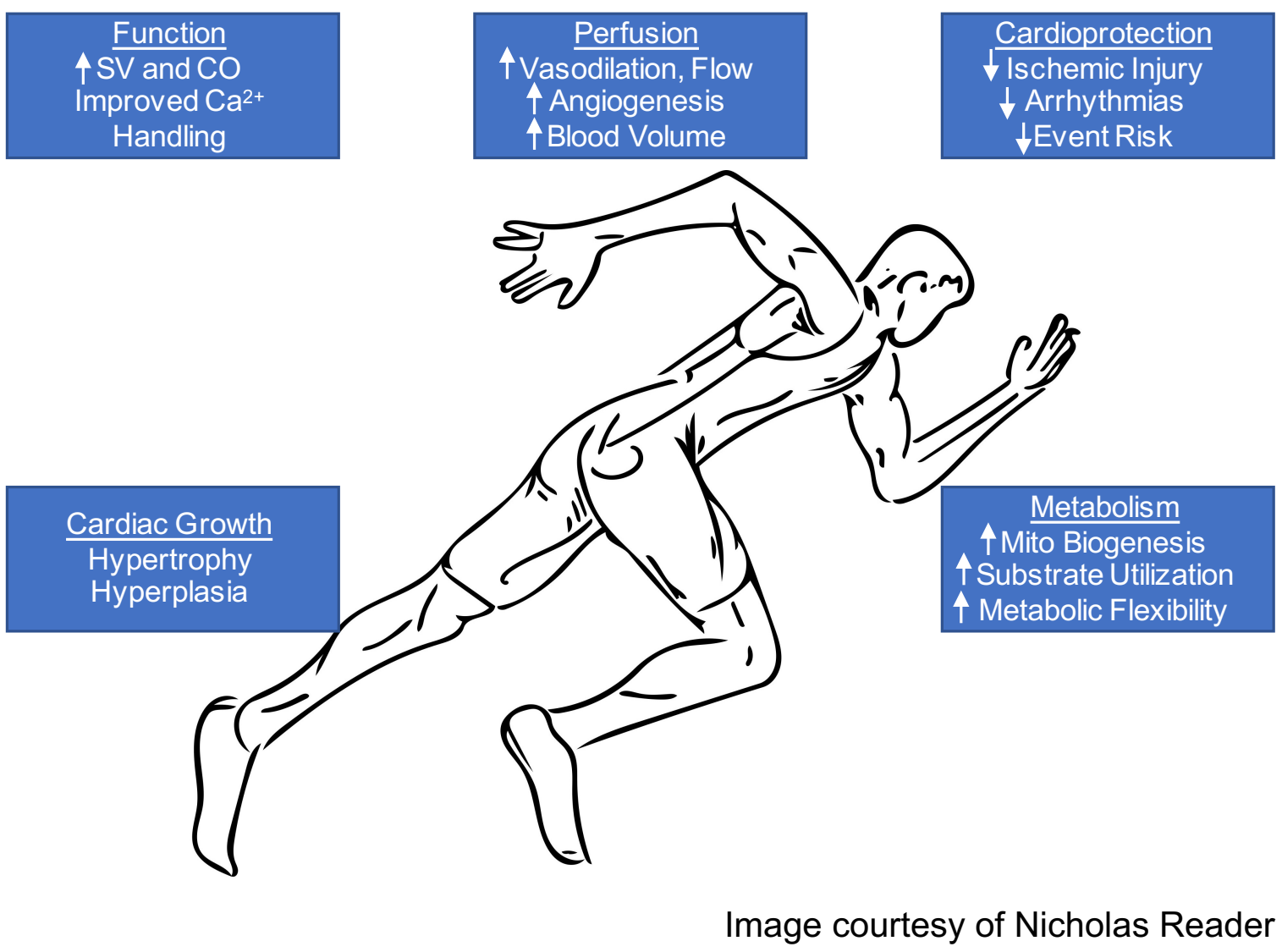


The heart is highly susceptible to changes in workload brought upon by exercise. Simply put, the primary response of the circulatory system is to increase blood flow to active areas (e.g. skeletal muscle) and to decrease it to non-working, less critical ones (e.g. GI tract). This is critical as the working muscle requires an increase in oxygen delivery to match the increase in metabolic demand required for the maintenance of contractile function. Not surprising, the heart is quite efficient at matching oxygen delivery to metabolic demand, resulting in little energetic waste. To accomplish this, the heart increases cardiac output, the product of heart rate $(\mathrm{HR})$ and stroke volume (SV). Several factors determine cardiac performance and are discussed below:

1) Heart rate: The single greatest factor determining cardiac output is heart rate. Under resting conditions, the human heart beats approximately 70 times per minute (bpm); however, during intense exercise this can increase nearly 3-fold to $200 \mathrm{bpm}$. Under parasympathetic control via the Vagus nerve, vagal tone is diminished during exercise and sympathetic stimulation predominates, leading to an increased HR. In addition to its significant contributions to cardiac output, heart rate is the most important factor determining myocardial oxygen consumption. This increase in oxygen utilization is essential to match the increase in metabolic demand. For this reason, increases in HR and cardiac output are tightly regulated to be directly proportional to the increase in oxygen requirements of not only the skeletal muscle, but the heart itself. Lastly, 
HRs are useful in estimating the myocardial metabolic cost of exercise. For example, the product of HR and systolic blood pressure is the ratepressure product (RPP), a rough index of the coronary blood flow myocardial oxygen consumption.

2) Preload: Several factors contribute to SV with preload being one of them. Preload, also known as end diastolic volume (EDV), is the volume of blood that stretches the ventricles to their greatest dimensions prior to contraction. Preload directly contributes to SV by means of the FrankStarling law. This law describes the positive relationship between SV and EDV and is driven by the length-tension relationship of the cardiomyocyte sarcomeres. As blood fills the ventricles, the myocyte myofibrils are stretched, creating more opportunities for cross-bridge interactions. An increased number of possible cross-bridges promotes greater contractile force, resulting in a larger volume of blood ejected, i.e., SV. Preload is directly related to the venous return of blood to the heart: its increase during exercise is caused by augmented skeletal and respiratory muscle pump function, higher venous tone (i.e. venoconstriction), and elevated cardiac output. Interestingly, it has been suggested that high heart rates may in fact decrease preload due to the loss of filling time; however, this remains controversial. ${ }^{14}$ 
3) Afterload: Unlike preload, afterload is referred to as a pressure and is negatively correlated to SV and cardiac output. Afterload is defined as the load which the ventricles must overcome to eject blood. The typical afterload (systolic blood pressure) in a healthy adult is $120 \mathrm{mmHg}$; however, during resistance exercise, such as weight lifting, arterial pressures can reach $480 \mathrm{mmHg}$ and is due to the arterial constriction of contracting skeletal muscle. ${ }^{15}$ These pressures, however, are not maintained as weight lifting is typically intermittent and dynamic. While this acute increase in afterload causes an immediately and relatively large decrease in SV, this leads to an increase in preload during the next cardiac cycle, increasing the effect size of Frank-Starling's law and increasing SV on the subsequent beat. While the increase observed with endurance exercise is much less severe $(\sim 100 \mathrm{mmHg})$, this increase in afterload is overcome by the heart due to the increases in preload and contractility.

4) Contractility: Contractility can be defined as the degree of force the heart muscle produces to eject blood and is positively correlated with SV. Unlike skeletal muscle, which is capable of recruiting additional fibers, the myocardium is fully engaged. Therefore, changes in contractility are completely dependent on intracellular $\mathrm{Ca}^{2+}$ availability, critical for muscle excitation-contraction coupling. When intracellular $\mathrm{Ca}^{2+}$ levels rise, $\mathrm{Ca}^{2+}$ binds to troponin- $\mathrm{C}$ causing a conformation change allowing actin- 
myosin cross-bridge interactions to occur. Utilizing ATP, the actin and myosin filaments slide past each other, shortening the sarcomeres and contracting the cell. Increased sympathetic stimulation and circulating catecholamines released during exercise increase intracellular $\mathrm{Ca}^{2+}$, resulting in enhanced myocardial contractility during exercise.

Similar to the effects on skeletal muscle, ${ }^{8}$ the plasticity of the heart allows for adaptation to repetitive bouts of exercise. This adaptive state following training elicits significant cardioprotection from cardiac insults including myocardial ischemia, ${ }^{16,17}$ pressure-overload (i.e. TAC), ${ }^{18}$ and diabetic cardiomyopathy.${ }^{19}$ Part of this cardioprotection is associated with improvements in cardiac function. The exercise-adapted heart, also known as athlete's heart, is associated with modestly enhanced diastolic function, whereas systolic function is primarily unaffected. ${ }^{20}$ This improvement in diastolic function can be linked back to those factors affecting preload. Endurance exercise training has been well documented to result in hypervolemia ${ }^{21}$ which contributes to increases in preload and cardiac output. Additionally, exercise reduces not only resting HR, but also the HR achieved at submaximal workloads, providing additional filling time for preload. ${ }^{14}$ The chronic effects of exercise on decreasing afterload in heathy individuals are not as large, as systolic blood pressures are only slightly reduced; however, in hypertensive individuals, exercise has a slightly greater effect size. ${ }^{22}$ At the cellular level, enhanced cardiac contractility contributes significantly to the improvement in cardiac performance. In exercise-trained rodents, fractional shortening improves 
$40-50 \%$ while relaxation rates improve $20-40 \% .{ }^{23}$ These improvements are, in part, explained by an increase in myofilament sensitivity to $\mathrm{Ca}^{2+} \cdot{ }^{24}$ Additionally, exercise has been shown to increase the activity of SERCA2a and phospholamban, contributing not only to enhanced $\mathrm{Ca}^{2+}$ release required for excitation-contraction coupling but also $\mathrm{Ca}^{2+}$ reuptake into the sarcoplasmic reticulum for relaxation. ${ }^{23,25}$ In addition to improving $\mathrm{Ca}^{2+}$ handling, regular exercise promotes cardiac growth, also referred to as physiological hypertrophy. This form of remodeling is characterized by a $10-20 \%$ increase in cardiac mass. Endurance exercise selectively induces an eccentric form of hypertrophy, wherein an increase in left ventricular (LV) volume is met with an equal increase in LV and septal wall thicknesses, coordinated by an increase in cardiomyocyte width and length. This is in stark contrast to a pathologic form of hypertrophy, where a much larger increase in LV volume is accompanied by a preferential myocyte lengthening, leading to wall thinning. Concentric hypertrophy, more prominent in pathology due to chronic elevations of afterload, is characterized by wall thickening as cardiomyocytes increase in width more so than length, reducing LV volume. While some strenuous exercise, such as weight training may induce a milder form of concentric hypertrophy, it is not believed to pathologic. ${ }^{26,27}$ Also, different from pathological hypertrophy is the observation that following only a few weeks of detraining in college and Olympic athletes, physiological hypertrophy regresses, indicating exercise-induced cardiac growth is a reversible phenotype. ${ }^{28,29}$

Other important factors also clearly distinguish these different forms of hypertrophy. For example, physiologic and pathologic hypertrophy differ 
significantly in their signaling mediators and transcriptional responses. In particular, while pathological hypertrophy is associated with the induction of the fetal gene program (e.g. ANP, BNP, $\beta-M H C$, smooth muscle $\alpha$-actin), physiological hypertrophy is not. ${ }^{30}$ Additionally, the molecular signaling events driving physiologic growth of the myocardium following exercise training are distinct. The most well established signaling and transcriptional events governing exerciseinduced physiologic growth are discussed below and in Fig. 2:

1. IGF1: Insulin-like growth factor 1 (IGF1), and its receptor (IGF1r) is the best described and potentially most important signaling event governing exercise-induced cardiac growth. Acute exercise increases the release of growth hormone $(\mathrm{GH})$ from the anterior pituitary gland which acts distally on the liver to increase IGF1 production. ${ }^{31}$ Additionally, exercise increases local IGF1 production in several target tissues, including the heart, thereby acting in an autocrine and/or paracrine fashion. ${ }^{32}$ Several transgenic mouse models have demonstrated the critical role of IGF1 in regulating physiologic cardiac growth. Both Igf1- and Igf1r1-null mice display a growth retardation phenotype with high perinatal mortality ${ }^{33}$ while Igf1 overexpressing mice display a physiologic form of cardiac hypertrophy. ${ }^{34}$ Furthermore, cardiomyocyte-specific deletion of $\operatorname{lgf1r} 1$ in adult mice prevents exercise-induced cardiac growth. ${ }^{35}$ These results clearly demonstrate the importance of IGF1 signaling in not only exercise-induced cardiac growth, but perinatal development as well. 
Figure 2: Exercise-mediated cardiac adaptive signaling pathways. Signaling pathways involved in the adaptation to exercise training. Physiologic growth is initiated by IGF1 through the IGF1/PI3K/AKT axis. Activation of AKT downregulates Cebpb transcription, allowing the upregulation of CITED4. This cascade leads to the upregulation of gene sets specific to promote cardiomyocyte growth. Inactivation of PTEN is also associated with the upregulation of the IGF1/PI3K/AKT signaling cascade. Nrg1, through ErbB, also activates AKT and leads to adaptive signaling events. Mitochondrial biogenesis also occurs through the IGF1/PI3K axis that may or may not involve AKT signaling. However, AKT signaling does upregulate the transcription of $P g c 1 \alpha$, resulting in the upregulation of adaptive metabolic gene sets. Catecholamine surges during exercise activate $\beta$-adrenergic receptors, leading to eNOS activation and the production of NO to increase cardiac perfusion. AMPK, an cellular sensor of the energy state of the cell, is also involved in the activation of eNOS as well as AKT, contributing to the overall adaptive response. Abbreviations: IGF1, insulin-like growth factor-1; IGF1r, insulin-like growth factor-1 receptor; Nrg1, neureglin1; ErbB, member of epidermal growth factor receptors; $\beta-A R$, beta-adrenergic receptor; $\mathrm{PI} 3 \mathrm{~K}$, phosphoinositide3 kinase; PTEN, phosphatase and tensin homolog; Akt, protein kinase B; C/EBP $\beta$, CCAAT/enhancer binding protein $\beta$; Cited4, cbp/p300-interacting transactivator

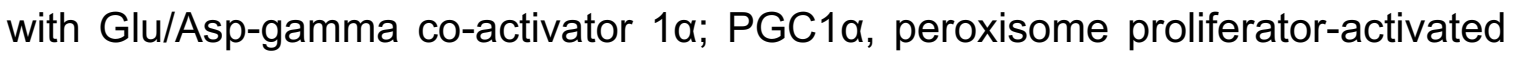
receptor gamma coactivator 1-alpha; eNOS, endothelial nitric oxide synthase; NO, nitric oxide; AMPK, AMP-activated protein kinase. 
FIGURE 2

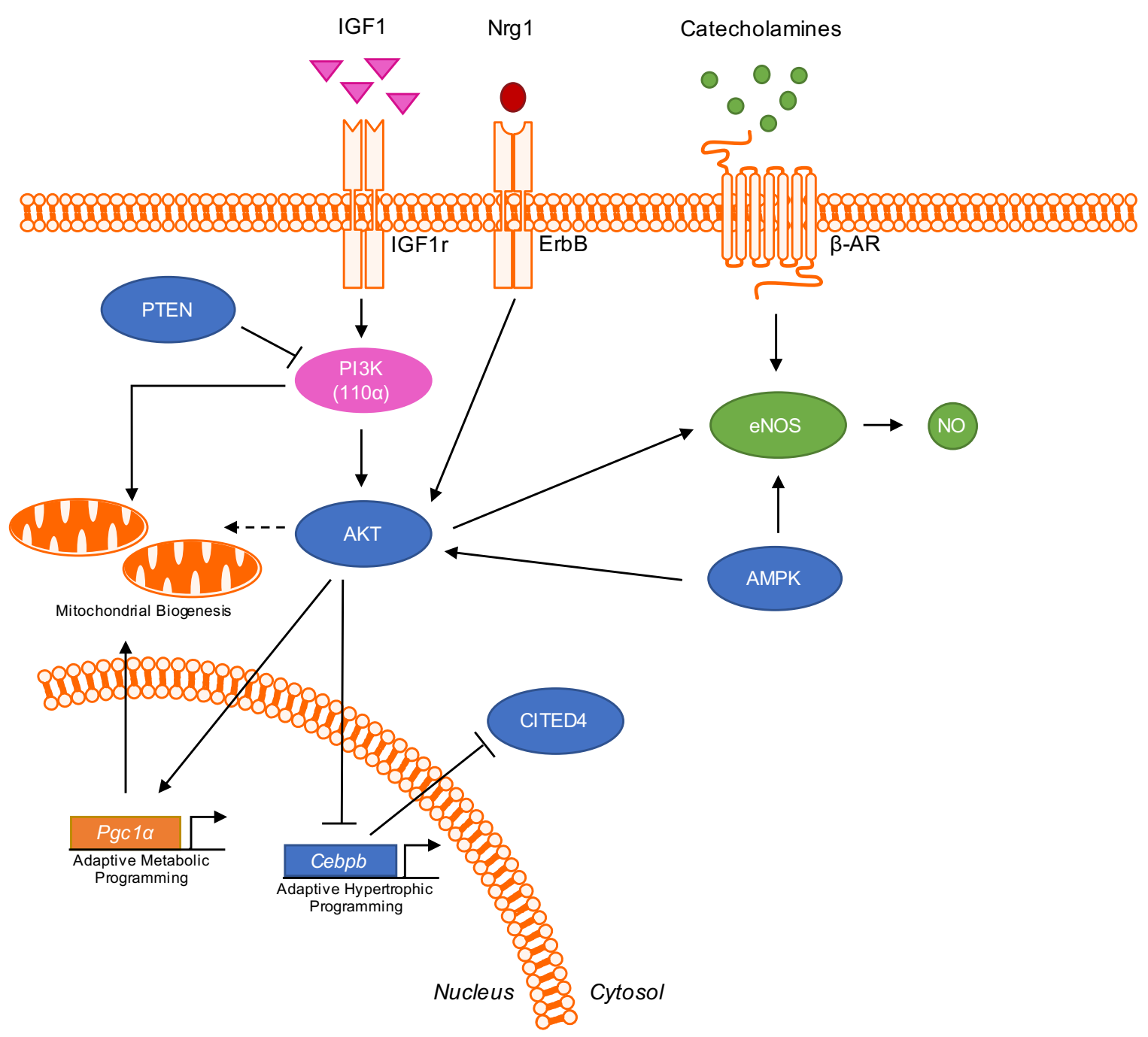


2) PI3K: IGF1r phosphorylation causes the activation of PI3-kinase, an intracellular family of signal transducing enzymes. Specifically, the activity of the PI3K (110 $\alpha)$ isoform appears to be necessary and sufficient for IGF1-mediated physiologic growth, as expression of a cardiac-specific dominant-negative PI3K (110 a) prevented exerciseinduced cardiac growth in mice. ${ }^{36}$ Conversely, constitutive activation of PI3K (110 $\alpha)$ in the heart promotes a physiologic form of hypertrophy. ${ }^{36,}$ ${ }^{37}$ In addition to the direct role of PI3Ks in regulating cardiac growth, deletion of Pten (phosphatase and tensin homolog), which dephosphorylates PI3Ks, has also been shown to mediate cardiac growth. ${ }^{38}$ Thus, the signaling events propagated by $\mathrm{PI} 3 \mathrm{~K}$, and its regulation by PTEN, are critical for the physiologic growth response.

3) AKT: The serine/threonine-specific protein kinase B, also known as AKT, also plays a critical role in physiologic hypertrophy. Functioning downstream of $\mathrm{PI} 3 \mathrm{~K}$, exercise training enhances the phosphorylation state of AKT at both the threonine 308 and 473 residues. $^{35,39-42}$ This activation of AKT is required for the hypertrophic response as mice with genetic deletion are resistant to exercise-induced cardiac growth. ${ }^{43}$ Interestingly, cardiac-specific overexpression of a constitutively active form of AKT, while initially promoting a physiologic form of hypertrophy, progressed to a pathologic form, suggesting that duration, and likely also intensity, determine the cardiac response to AKT signaling. ${ }^{44}$ 
4) C/EBPB and CITED4: Recent studies have identified transcriptional events downstream of AKT activation that are essential for exerciseinduced physiologic growth. For example, exercise training was shown to downregulate cardiomyocyte expression of the CCAAT/enhancer binding protein- $\beta$ (C/EBP $\beta)$, in an AKT-dependent manner. In that study, cardiomyocyte-specific overexpression of C/EBP $\beta$ resulted in physiological hypertrophy and resistance to the pathologic response to pressure overload. ${ }^{45}$ Additionally, the exercise-induced transcriptional downregulation of Cebpb led to the transcriptional upregulation of Cited $4,{ }^{45}$ and overexpression of Cited 4 was show to be sufficient to drive physiologic cardiac growth and promote recovery from ischemic injury. ${ }^{46}$ While C/EBP $\beta$ and CITED4 are important for mediating the response to exercise, additional mechanisms of activation and downstream targets require further investigation.

5) Additional effectors of physiologic cardiac growth: Several other factors have been shown to be involved to some degree in the response to exercise. Nueregulin 1 signaling through the ErbB family of tyrosine kinase receptors produces similar effects to that observed in the exercise-adapted heart, in part, through the activation of AKT. ${ }^{47}$ AMPactivated protein kinase (AMPK), important for its role in cellular energy homeostasis, has been suggested to play a role in the development of both physiologic and pathologic hypertrophy. ${ }^{27}$ Additionally, signaling 
though MAPK (mitogen-activated protein kinase) ${ }^{48}$ and mTOR

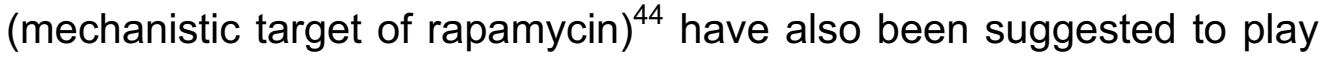
roles in governing the cardiac hypertrophic program.

Recent evidence suggests that cardiomyocytes, once thought incapable of regeneration and proliferation, may in fact do so at a low rate in the adult heart. ${ }^{49-}$ ${ }^{51}$ Increasing lines of evidence also suggest that exercise not only stimulates cardiomyocyte hypertrophy, but potential hyperplasia as well. Rodents subjected to exercise show elevated makers of proliferation such as PCNA expression, Ki67 positivity, and BrdU incorporation in the heart along with the activation of $\mathrm{c}-\mathrm{kit}^{+}$and Sca- $1^{+}$cells, believed to be cardiac progenitor cells. ${ }^{45,52-54}$ While these results suggest that exercise-activated cardiomyocyte proliferation may contribute to physiological growth, more detailed studies, such as cell fate-mapping are required before more definitive conclusions can be drawn.

As cardiomyocytes grow in response to exercise, several other adaptive responses, both intrinsic to the cardiomyocyte and within the myocardium as a whole, must occur as well. One such response is an enhancement of cardiac perfusion. This is a critical adaptive response as both increases in workload and a larger myocyte both have a higher energy and oxygen demand. This increase in cardiac perfusion occurs primarily through two modes of action, vasodilation and angiogenesis. First, exercise results in the release of catecholamines which, in addition to their effect on $\mathrm{Ca}^{2+}$ dynamics and contractility, increases the phosphorylation of endothelial nitric oxide synthase (eNOS) ${ }^{55}$ Enhanced eNOS 
activity increases nitric oxide (NO) bioavailability, a potent vasodilator. This effect is also driven by the exercise-induced activation of AKT and AMPK, both of which promote the phosphorylation of eNOS. ${ }^{56,57}$ Secondly, chronic exercise training induces an angiogenic response within the myocardium, resulting in de novo capillary formation. ${ }^{58}$ This angiogenic response is in part associated with exercisemediated increases in VEGF secretion ${ }^{59}$ and circulating angiogenic progenitor cells. $^{60}$

The most common metabolic adaptation of the heart to exercise is mitochondrial biogenesis. ${ }^{61-63}$ While various signaling events likely lead to an increase in mitochondrial mass, exercise-induced increases in peroxisome proliferator-activated receptor $\gamma$ coactivator $1 \alpha$ (PGC-1a) play a critical role. ${ }^{64}$ Interestingly, induction of PGC-1 $\alpha$ in response to exercise was prevented in IGF1r knockout mice, and it has been shown that signaling through PI3K, but not AKT, is required for this biogenic response. ${ }^{35,65}$ Accompanying the increase in mitochondrial mass, respiratory function and capacity appears to be enhanced in the physiologically hypertrophied heart. In both isolated permeabilized cardiac muscle fibers ${ }^{65}$ and the isolated perfused heart, ${ }^{40,66}$ exercise training increased the rate of mitochondrial fatty acid oxidation (FAO). This is in part due to the increase in mitochondrial mass, but also the upregulation of genes associated with $\mathrm{FAO}^{67}$

In addition to FAO rates, others have shown an increase in glucose oxidation (GO) as well. ${ }^{66}$ This enhanced metabolic capacity of the exercised heart is a hallmark characteristic, distinguishing it from pathologic forms of hypertrophy 
where it is well accepted that metabolic flexibility and energy homeostasis are compromised. Therefore, studying the metabolic phenotype of the physiologically hypertrophied heart may provide therapeutic avenues to treat or delay the onset of maladaptive pathologic remodeling. In the following section, the acute and chronic effects of exercise on cardiac metabolism will be discussed in detail.

\section{Cardiac Metabolism in Exercise}

The heart has an incredibly high energy demand, which requires continuous adenosine triphosphate (ATP) generation to sustain contractile function, ionic homeostasis, metabolic processes, and signaling. While myocardial ATP turnover is high $\left(\sim 30 \mu \mathrm{mol} \bullet \mathrm{g}\right.$ wet $\left.\mathrm{wt}^{-1} \bullet \mathrm{min}^{-1}\right)$, ATP content is relatively low $(\sim 5 \mu \mathrm{mol} \bullet \mathrm{g}$ wet $\mathrm{wt}^{-1}$ ) resulting in complete turnover approximately every $10 \mathrm{~s}^{68-70}$ The heart fuels this turnover by generating $>95 \%$ of its ATP from mitochondrial oxidative phosphorylation with the remaining $5 \%$ derived primarily from glycolysis. ${ }^{70,} 71$ Although the majority (60-70\%) of the ATP generated supports contractile function, $30-40 \%$ is necessary to maintain ionic homeostasis through ion pumps, specifically the $\mathrm{Ca}^{2+}$-ATPase in the sarcoplasmic reticulum. ${ }^{72,73}$

Basally, it is well accepted that the oxidation of free fatty acids (FFA) contributes $70-90 \%$ to oxidative phosphorylation with glucose, glycogen, lactate, ketones, and amino acids contributing the remaining $10-30 \%$. With the ability to utilize a myriad of substrates, the heart is considered a metabolic "omnivore", utilizing whichever substrates are available at a given time; however, with multiple substrates consistently available for use, competition for utilization exists. The 
competition of substrates has long been demonstrated in both in vivo arterialcoronary sinus difference studies ${ }^{74-77}$ and perfused heart preparations ex vivo. ${ }^{78}$, 79 The first observation of substrate competition was observed in the isolated perfused heart by Philip Randle in $1963^{80}$ In this study, Randle demonstrated that increasing delivery of FFAs to the heart increased FAO and decreased glucose to pyruvate conversion and oxidation. Interestingly, exercise is also associated with the competition of substrates for utilization.

Acute exercise (i.e. an acute increase in workload) has a robust effect on not only cardiac metabolism. For example, it is estimated that TCA cycle flux increases 70 - to 100 -fold in an effort to match energy production to the large increase in consumption in working muscle. ${ }^{81}$ In the heart, exercise increases LV contractile power and myocardial oxygen consumption 4- to 6-fold above resting rates, which requires a significant increase in the generation of $\mathrm{NADH}$ and $\mathrm{FADH}$, from substrate oxidation. ${ }^{82}$ Several ex vivo ${ }^{78,83}$ and in vivo ${ }^{84-87}$ studies demonstrate that an increase in myocardial workload is accompanied by significant, yet selective, increases in the metabolism of various substrates. This increase in myocardial metabolism is accomplished by increased substrate availability and delivery to the myocardium as well as through extensive intracellular signaling that regulates substrate selection and utilization, coordinating myocardial metabolism. The effects of exercise on individual substrate metabolism is discussed below and in Fig. 3: 
Fatty Acids: As already mentioned, the oxidation of FFA contributes the most to energy provision in the normal healthy heart. Because of their hydrophobic properties, FFA are bound to albumin for transport in circulation at a concentration of $0.2-0.6 \mathrm{mM} ;{ }^{88,89}$ however, during an acute bout of exercise, these levels can increase to $2.4 \mathrm{mM}^{89}$ Because the availability of circulating FFAs is a primary determinant of their uptake by the myocardium, ${ }^{74}$ the exercising heart demonstrates enhanced rates of FFA uptake. ${ }^{90}$ This increase in circulating FFAs during exercise is primarily due to elevations in catecholamines, which activate hormone sensitive lipase in adipose and skeletal muscle tissues to de-esterifies FFAs and promote their release into circulation. ${ }^{91}$ Exercise likely also facilities the translocation of fatty acid translocase (FAT)/CD36, the primary protein carriermediated transporter for FFA uptake, as it has been shown that contracting skeletal muscle acutely increased its localization to the plasma membrane; ${ }^{92,} 93$ however, this effect has yet to be confirmed in the heart.

Following uptake, FFAs are esterified to fatty acyl-coenzyme $A$ (CoA) by fatty acyl CoA synthase and either transported into the mitochondria for eventual beta-oxidation or used for the synthesis of triacylglycerides (TAGs). At rest, human isotopic labeling studies measuring ${ }^{14} \mathrm{CO}_{2}$ production in coronary venous blood indicate that $84 \%$ of the FFA taken up by the cell are immediately oxidized, leaving $\sim 26 \%$ available for entry into the TAG pool. ${ }^{94}$ This contribution to the TAG pool is not trivial, as intramyocardial TAG turnover occurs in as little as $5 \mathrm{~h}$ and contributes $10 \%$ to the total energy expenditure of the heart. ${ }^{82,}{ }^{95}$ Interestingly, myocardial TAG utilization rates were found to be increased considerably under exercise conditions 
as measured by a significant release of free glycerol, suggesting increased hydrolysis of intramyocardial stores. ${ }^{87}$ In an ex vivo heart preparation, this increase in TAG turnover was a function of increased lactate availability, suggesting that lactate, which is elevated during exercise, stimulates FAO and the turnover of TAG stores. This conclusion was made by the observation that lactate in the perfusion buffer increased intracellular fatty acid content and free glycerol release, while maintaining TAG levels in the myocardium. ${ }^{96}$ With respect to FAO rates, elevated circulating FFA levels, as occurs during exercise, enhances myocardial utilization for beta-oxidation and ATP production as indicated by isotopic labeling studies measuring ${ }^{14} \mathrm{CO}_{2}$ production. ${ }^{94}$

Lactate: While the heart produces a considerable amount of lactate at rest, ${ }^{14} \mathrm{C}$ lactate tracer studies calculating arterial-coronary sinus differences indicate the heart consumes an even greater amount of lactate, making the heart a net lactate consumer. ${ }^{77,90,97,98}$ Myocardial lactate uptake, which is positively correlated with arterial lactate concentration, is almost entirely used for oxidation. ${ }^{84}$ Under conditions of elevated arterial concentrations (e.g. exercise), lactate oxidation is significantly enhanced. ${ }^{79,85}$ This increase in lactate oxidation appears to be correlated with exercise intensity, which determines arterial lactate levels. Under lower exercise intensities, the contribution of lactate oxidation to myocardial oxidative metabolism increased from $13 \%$ to $28 \%$ and was considerably higher than glucose oxidation (8-14\%) ${ }^{84}$ However, during intense exercise lactate levels can increase 10-fold, from $1 \mathrm{mM}$ to $10 \mathrm{mM}$, and it has been suggested that the 
Figure 3: Myocardial metabolism during exercise. Schematic of cardiac metabolism in exercise. Acute exercise results in changes in the bioavailability and utilization of both external (arterial concentrations) and internal (intracellular storage depots) energy substrates for ATP production. Arrows are indicative of changes in both bioavailability and utilization by the myocardium during exercise. For diagrammatic simplicity, the mitochondrion is shown as a single membrane, with CPT1/2 shown as an aggregate of the canitinylation and reCoAcylation steps required for entry of fats into the mitochondrion for beta oxidation. Similarly, as several GLUT and MCT isoforms are present in the heart, they are diagrammatically represented as a family of transporters. Abbreviations: FFA, freefatty acids; FAT/CD36, fatty acid translocase; GLUTs, glucose transporters; MCTs, monocarboxylate transporters; G6P, glucose-6-phosphate; F6P, fructose-6phosphate; $\quad F-1,6-P_{2}, \quad$ fructose-1,6-bisphosphate; $F-2,6-P_{2}, \quad$ fructose-2,6bisphosphate; GAP, glyceraldehyde-3-phosphate; DHAP, dihydroxyacetone phosphate; PFK1, phosphofructokinase 1; PFK2, phosphofructokinasebisphosphatase 2; LDH, lactate dehydrogenase; PDC, pyruvate dehydrogenase complex; TAG, triacylglyceride; CPT1,2, carnitine palmitoyl transferase 1 and 2. 
FIGURE 3

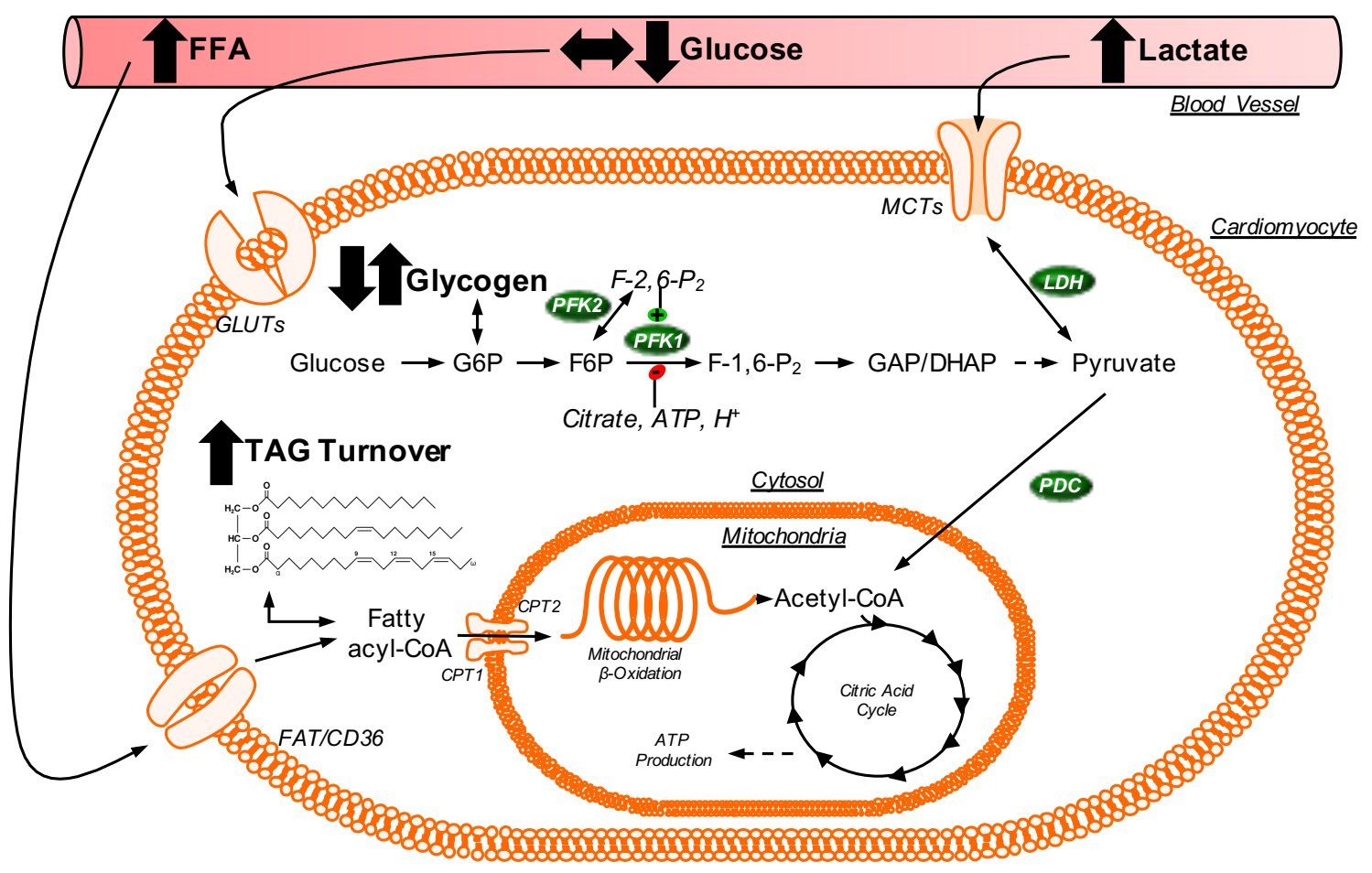


contributions of lactate to total oxidative metabolism under these conditions may in fact be much higher, accounting for $60-90 \%$ of substrate utilization. ${ }^{85,99-101}$

The effects of exercise training on myocardial lactate metabolism are not well known and is complicated by the fact that a primary effect of training is the reduction of arterial lactate levels for a given sub-maximal intensity. ${ }^{102}$ Because lactate utilization is a consequence of its availability, this would suggest that lactate oxidation and its contribution to myocardial oxidative metabolism may be diminished in the trained heart. However, it is likely that training results in metabolic adaptations similar to those seen for fatty acid metabolism. For example, recent studies have suggested that training increases LDH activity, ${ }^{103}$ decreases the $\mathrm{Km}$ for conversion of lactate to pyruvate, ${ }^{104}$ and increases the monocarboxylic transporter (MCT1) responsible for lactate uptake, ${ }^{105}$ all of which would contribute to enhanced lactate oxidation at a given concentration. Often rarely considered as a myocardial substrate, the importance of lactate to energetics and performance should not be understated. In a recent study by Levy et. al., systemic lactate deprivation resulted in cardiac energy deprivation and dysfunction, ${ }^{106}$ highlighting the importance of lactate as an energy source.

Glucose: Under resting conditions, glucose is the most abundant substrate available to the myocardium with a circulating concentration of $4-5.5 \mathrm{mM}$ in healthy individuals. While availability is not an issue, the heart preferentially utilizes FFA for oxidative metabolism with contributions of glucose equal to that of lactate $(\sim 17 \%) .{ }^{107}$ This is somewhat surprising considering that glucose is a much more 
efficient carbon source than FFAs, indicated by the phosphate to oxygen ratio $(\mathrm{P} / \mathrm{O})$ for complete oxidation of substrates, ${ }^{108}$ which would make glucose a more efficient source of energy during increased workloads. As with FFAs and lactate, glucose uptake by the myocardium is also dependent on arterial concentrations; however, arterial glucose concentrations are tightly controlled and do not generally fluctuate. During highly intense (e.g. weightlifting) and prolonged endurance exercise, arterial glucose concentrations have been shown to diminish, ${ }^{89}$ while others report that high intensity aerobic exercise resulted in an increase in blood glucose ${ }^{86}$ Interestingly, this increase in arterial glucose was not associated with an increase in glucose uptake indicated by positron emission tomography utilizing $2-\left[{ }^{18}\right.$ F]fluoro-2-deoxy-D-glucose; however, during lower intensities glucose uptake was increased with no change in arterial levels. ${ }^{86}$ These results of glucose uptake under lower intensities were in agreement with a previous report by Gertz et al. ${ }^{84}$ however, others have reported diminished glucose uptake during exercise. ${ }^{87,} 109$

Glucose uptake is facilitated by protein carrier-mediated transport through a family of glucose transporters (GLUT), with the insulin-dependent GLUT4 as the most abundant in cardiac tissue. ${ }^{110}$ Exercise increases the translocation of GLUT4 to the cellular membrane in part by catecholamine activated $\beta$-adrenergic signaling ${ }^{111,} 112$ and through the exercise-induced activation of AMPK. ${ }^{113}$ Interestingly, this translocation appears to be insulin-independent as circulating insulin levels are diminished during exercise. ${ }^{114}$ Activation of AMPK also increases phosphorylation of phosphofructokinase 2 (PFK2) at the S483 residue, which increases the levels of fructose-2,6-bisphosphate $\left(\mathrm{F}-2,6-\mathrm{P}_{2}\right)$, a potent activator of 
PFK1 activity in the heart. If this occurs in the exercising heart, glycolytic rates would likely be elevated. Interestingly, increased utilization of glucose during exercise does not increase lactate release from the myocardium but rather promotes enhanced glucose oxidation, ${ }^{84}$ in part, by increasing muscle pyruvate kinase activity. ${ }^{115}$ This would suggest that a primary effect of exercise is to increase the coupling of glycolysis to glucose oxidation. Improved coupling of glycolysis and glucose oxidation, especially if glucose uptake is diminished during exercise, would be advantageous as it would increase the proportion of glucose that contributes to energy production.

Another source of glucose for the myocardium is the intracellular storage of glycogen. Although the glycogen pool is only $20 \%$ of that of skeletal muscle, myocardial glycogen turnover can be high. ${ }^{116}$ Interestingly, a small amount of glucose that enters the glycogen pool is immediately cycled back out for eventual conversion to pyruvate $;^{117}$ the significance of this cycling, however, and its relevance in exercise, is not well understood. The effects of glycogen content are more well defined under increased workloads. Acute increases in work stimulated by epinephrine increase glycogen breakdown via glycogen phosphorylase ${ }^{83,117}$, 118 which is preferentially used for oxidation rather than lactate production as shown by isotopically $\left({ }^{13} \mathrm{C}\right)$ saturating the glycogen pool. ${ }^{118,}{ }^{119}$ Conversely, glycogen synthesis by glycogen synthase, increases during fasting ${ }^{120-122}$ and when competing substrates (FFA and lactate) predominate in their oxidation. ${ }^{123,} 124$ Because catecholamines, FFA, and lactate are all elevated during exercise, the effects of exercise on glycogen content are likely subject to duration, intensity, and 
the time at which such measurements are made. For instance, it is likely that at the onset of exercise, prior to an increase in substrate availability, glycogen is broken down to meet the abrupt increase in ATP demand. Once arterial FFA and lactate levels rise over time, glycogen breakdown is inhibited and synthesis occurs. Because of these competing effectors of glycogen metabolism, a well-controlled study is required to fully understand the effects of exercise intensity and duration on glycogen metabolism.

Ketones and Amino Acids: Ketone bodies, acetoacetate and $\beta$ hydroxybutyrate, can contribute to oxidative metabolism through their conversion to acetyl-CoA, and similar to FFA and carbohydrates, uptake and oxidation is positively correlated to their arterial concentration. ${ }^{125}$ However, ketone bodies themselves are insufficient to meet the energetic need of the heart, both in vivo ${ }^{126}$ and ex vivo. ${ }^{127}$ While ketone bodies may play a significant role in energy provisions in the pathologic heart, ${ }^{128}$ their contributions to the exercising and physiologically hypertrophied heart are likely minimal.

Amino acids play an important role in contributing several carbon-based intermediates to the TCA cycle. For instance, alanine can undergo transamination to form pyruvate for oxidation, glutamine can enter at the point of $\alpha$-ketoglutarate, and aspartate can be converted to oxaloacetate, all providing TCA intermediates. Additionally, the malate-aspartate shuttle is important in transferring the reducing equivalent $\mathrm{NADH}$ generated from cytosolic glycolysis and lactate to pyruvate conversion, into the mitochondria for the ETC. This reaction in turn regenerates 
cofactor levels of $\mathrm{NAD}^{+}$, required for glycolysis and LDH activity. In the working heart, only leucine, a branched chain amino acid, has been shown to contribute to oxidative substrate utilization, albeit to a minimal $(0.4 \%)$ degree. ${ }^{129}$ Similar to ketone bodies, amino acid metabolism in the context of exercise is not well understood.

While understanding how exercise affects the metabolism of individual substrates provides invaluable information, integration of the metabolism of all substrates becomes much more complex, but provides a more complete picture. A more complete and thorough interrogation of myocardial metabolism can be obtained by measuring the utilization of all substrates and the regulation each imposes on the other. For example, in a study by Schonekess, hearts perfused at a constant workload had significantly diminished contributions of glycolysis and glucose oxidation to ATP production when FFA levels were increased to a physiologic range associated with exercise; interestingly, this observation was also made when lactate in the perfusion buffer was increased to physiologic exercising levels. ${ }^{79}$ In a similar ex vivo study, workload was increased by epinephrine in the presence of exercising levels of lactate and FFAs, which blunted the increase in glucose utilization. ${ }^{78}$ Similar results from in vivo studies further support that although an increase in cardiac workload is typically associated with an increase in glucose uptake and oxidation in the myocardium, ${ }^{78,83,84,86}$ when concentrations of lactate and FFAs were increased, glucose uptake and oxidation were diminished. $^{78,86,87,109}$ 
The diminishment in glucose utilization most likely occurs through allosteric inhibition of key glucose-metabolizing enzymes. For instance, acetyl-CoA derived from the oxidation of FFA in the mitochondria inhibits PDH activity, reducing the oxidation of glucose-derived pyruvate. Furthermore, the subsequent increase in citrate is inhibitory to the rate-limiting and committed step of glycolysis, phosphofructokinase 1 , and to a lesser extent glucose uptake. The magnitude of inhibition increases along the glycolytic pathway with PDH inhibition being the most severe and glucose uptake the least. ${ }^{130,131}$ Lactate oxidation also acts to inhibit glycolytic flux by reducing the bioavailability of $\mathrm{NAD}^{+}$, a cofactor for

glyceraldehyde-3-phosphate dehydrogenase, and decreasing $\mathrm{H}^{+}$extrusion which is inhibitory to PFK1. Collectively these results suggest that during exercise, when arterial lactate and FFAs increase, glucose utilization is likely diminished.

\section{Metabolism as a Contributing Factor to Growth}

In tissues such as skeletal muscle, adaptive changes in gene expression are thought to be initiated by the repetitive bouts of metabolic stress triggered by strenuous physical activity. ${ }^{8}$ As acute and chronic exercise alter cardiac metabolism, it could be hypothesized that these changes could contribute to the adaptive response; however, understanding the mechanisms and pathways involved is difficult due to the complex and integrative nature of metabolism. Of particular interest is the metabolism of glucose via glycolysis, which is highly regulated during exercise. A central pathway of intermediary metabolism, the fate of glucose is not only limited to pyruvate or lactate for energy production, but it also 
contributes to several ancillary pathways of glucose metabolism, such as the pentose phosphate pathway (PPP) and the glycerolipid synthesis pathway (GLP). These pathways provide the first rationale for how changes in glucose utilization could contribute to the cardiac adaptation to exercise.

The simplest explanation as to why changes in metabolism would precede and contribute to cardiac growth is by providing a material cause necessary for that growth. This material cause comes in the form of the DNA, amino acids, proteins, and phospholipids that are required for cellular growth. For example, in the oxidative phase of the PPP, the metabolism of glucose-6-phosphate (G6P) reduces $\mathrm{NADP}^{+}$to NADPH which is required for glutathione recycling and anabolic reactions. End products of the non-oxidative phase, however, are critical in the generation of ribose 5-phosphate, the precursor for purine and pyrimidine nucleotide biosynthesis. These nucleotides are the organic structure serving as the basis for the synthesis of DNA, RNA, and nucleic acids, without which growth cannot occur. In the heart, the PPP is activated during pathological hypertrophy ${ }^{132-}$ ${ }^{134}$ and heart failure, ${ }^{135}$ and modulating pathway activity regulates the severity of cardiac pathology. ${ }^{136-141}$ Similarly, growth of a cell cannot occur without expansion of the cellular membrane which requires increased bioavailability of phospholipids derived from the GLP. Although less is known about the GLP, increased pathway activity may in fact contribute to pathologic cardiac hypertrophy. ${ }^{142}$ In the exercising heart, very little is known with regards to pathway flux through the PPP and GLP. However, during exercise when FFA and lactate oxidation increase, 
allosteric inhibition at the point of PFK1 may in fact coordinate flux through these pathways, as has been shown in several non-cardiac cell types. ${ }^{143-146}$

Another biosynthetic pathway branching off glycolysis is the hexosamine biosynthetic pathway (HBP), which could also be influenced by changes in PFK and glycolytic activity. Requiring a sugar donor, the synthesis of O-linked $\beta-N$ acetylglucosamine (O-GIcNAc), a monosaccharide donor for nuclear and cytosolic proteins, can alter protein function, gene transcription, and modulates cell survival under conditions of stress. ${ }^{147-150}$ While several molecular signaling pathways are well understood in the exercised heart, post-translational O-GIcNAcylation is a relatively new area of investigation. In rats, an acute bout of swim training resulted in a $30 \%$ increase in skeletal muscle UDP-hexosamine concentrations that persisted for $16 \mathrm{~h}$ following exercise; ${ }^{151}$ however, in mouse hearts following 15 mins of treadmill exercise, O-GIcNAc levels were diminished. Interestingly, in these same mice, de-O-GlcNAcylation of O-GlcNAc transferase (OGT), the enzyme responsible for catalyzing the addition of the GIcNAc moiety to proteins, resulted in dissociation from the repressor element 1 silencing transcription factor complex and initiation of physiological growth signaling. ${ }^{152}$ Following training, the physiological hypertrophied heart is associated with decreased O-GlcNAc levels and a reduced expression of several regulatory enzymes in the HBP. ${ }^{153,154}$ The results from these studies suggest that exercise-induced changes in metabolism and coordinate changes in HBP flux could contribute to the physiologic growth program. 
Another potential role for metabolism in regulating the cardiac adaptive response to exercise is through direct intracellular signaling of metabolites themselves. For instance, an increase in G6P levels in ex vivo hearts subjected to increased workload, which is likely to occur in vivo during exercise when glucose utilization rates are diminished, leads to the activation of mTOR. ${ }^{155}$ Another example of a metabolite acting in a direct signaling manner is 5-Aminoimidazole4-carboxamide ribonucleotide (AICAR), an intermediate of the PPP. An analog of adenosine monophosphate (AMP), AICAR stimulates AMPK, increasing its activation state. ${ }^{156}$ As mTOR and AMPK are implicated to play a role in the exercise-induced adaptation, metabolites as direct signalers may play a critical role in the activation of the physiologic growth program.

Based on the literature discussed within this section, there appears to be ample evidence to suggest that metabolism may play a contributing role to the adaptive response to exercise, preceding and triggering physiologic growth of the heart. This raises several questions: How does exercise acutely and chronically affect myocardial metabolism?; Do changes in metabolism contribute to the exercise-induced adaptation of the heart?; and if so, how does metabolism activate the programs of exercise-induced physiologic growth? These questions and fundamental concepts led to the development of the following studies, which sought to identify the role of metabolism in exercise-induced cardiac remodeling. 


\title{
CHAPTER II
}

\section{FVB/NJ MICE ARE A USEFUL MODEL FOR EXAMANING CARDIAC ADAPTATIONS TO TREADMILL EXERCISE}

\begin{abstract}
Introduction
Regular exercise improves cardiovascular health, ${ }^{5-7}$ augments musculoskeletal function, ${ }^{8}$ and increases both healthspan ${ }^{8-10}$ and lifespan. ${ }^{5,11-13}$ Nevertheless, the molecular mechanisms by which exercise promotes health are poorly understood. ${ }^{157}$ Exercise studies commonly use murine models, which are valuable for identifying critical gene programs that contribute to exercise adaptation, primarily, because they offer the benefit of relatively rapid and controlled genetic modification [e.g., ${ }^{25}, 40,158,159$ ]. Nevertheless, sources of variability in such studies could confound our understanding of how exercise mitigates disease or increases overall health. Minimization of confounding factors is an important consideration for designing exercise studies as well as for interpreting the results obtained.

Although mouse models cannot perfectly recapitulate the complex physiological changes occurring in humans with physical activity, they can phenocopy particular aspects of physiological adaptation. For this purpose, three
\end{abstract}


models of mouse exercise are used frequently: treadmill training, forced swimming, and voluntary wheel running. While each of these models have advantages and limitations, ${ }^{158,160}$ treadmill training provides the investigative advantage of controlling the amount of work performed in each training session, which is critical for understanding dependency of (patho)physiological adaptations on exercise workload and becomes increasingly important in studies of mice having different masses (e.g., obesity studies). Unlike the treadmill modality, work cannot be calculated easily in swimming or voluntary wheel exercise. For these reasons, treadmill training is a reliable, well-controlled, and often superior model of exercise for research studies. Nevertheless, several factors contribute to treadmill exercise (non)compliance and to exercise-induced adaptations. These include, but are not limited to, mouse strain, exercise environment, acclimatization, motivation, and assessments of exhaustion. ${ }^{52,161}$

Of these, mouse strain is of principal importance. Inbred strains of mice and rats have pronounced differences in their ability to exercise, or their choice to do so. ${ }^{162-166} \mathrm{~A}$ preponderance of genetic mouse models are on the $\mathrm{C} 57 \mathrm{BL} / 6 \mathrm{~J}$ or the FVB/NJ background ${ }^{167,} 168$; however, these strains show strikingly different preferences and capacities for exercise. For instance, C57BL/6J mice appear to be poor treadmill runners, yet display superior capacity on voluntary exercise wheels, while the opposite is true for FVB/NJ mice. ${ }^{163,166}$ The reason(s) for these differences in training modality preference remain unclear. One reason could relate to the fact that laboratory mice are primarily nocturnal animals, demonstrating the highest activity and food consumption during the night cycle ${ }^{169-171}$; however, to our 
knowledge, the impact of time of exercise (day or night) on treadmill exercise capacity and compliance has not been investigated. The goals of this study were: (1) to test for differences in treadmill exercise capacity and compliance between C57BL/6J and FVB/NJ mice; (2) to delineate whether diurnal or nocturnal training influences exercise compliance and adaptive responses to training; and (3) to assess systemic and cardiac-specific exercise adaptations in mice compliant with treadmill exercise protocols. Our study demonstrates that FVB/NJ mice are a superior strain for treadmill exercise and that the timing of treadmill exercise, at least in this strain, does not influence compliance or exercise-induced adaptation.

\section{Experimental Procedures}

Animals. All procedures were approved by the University of Louisville Institutional Animal Care and Use Committee. C57BL/6J and FVB/NJ mice were ordered from Jackson Laboratory (Bay Harbor, ME) at 12 weeks of age and allowed to acclimate at the University of Louisville animal facility for three weeks. At 15 weeks of age, male mice were assigned randomly by strain to sedentary (SED) or exercise (EXEDay or EXE-Night) groups. Food and water were provided ad libitum, and the mice were maintained on a 12:12-h light-dark schedule. Because the majority of published studies regarding exercise in mice have utilized male mice, we chose this gender for our study, which enables comparison with the literature. At the conclusion of the study and $24 \mathrm{~h}$ after the last exercise session, the mice were fully anaesthetized with sodium pentobarbital $(40 \mathrm{mg} / \mathrm{kg}$, i.p.), followed by euthanasia 
via excision of the heart. These procedures are consistent with the AVMA Guidelines on Euthanasia.

Metabolic phenotyping. To assess for differences in basal metabolism and diurnal/nocturnal behavior, metabolic cage analyses were performed in naïve 15week-old C57BL/6J and FVB/NJ mice, essentially as described. ${ }^{172,173}$ Body weight was recorded prior to the initial and final exercise capacity tests to assess for changes in total body mass. Oxygen consumption rates, carbon dioxide production rates, respiratory exchange ratios, food consumption, water consumption and activity (sum of ambulatory and fine movements) were measured using a physiological/metabolic cage system (TSE PhenoMaster System, Bad Homberg, Germany) as previously described. ${ }^{172,173}$

Exercise capacity testing. We performed exercise familiarization and capacity testing in a manner similar to that outlined previously, ${ }^{166}$ with minor modifications (Fig. 4A,B). Mice were familiarized to the motorized rodent treadmill (Columbus Instruments, Columbus $\mathrm{OH}$ ) on the Wednesday and Thursday before the first week of training. Familiarization consisted of an initial 10 min period where the treadmill speed and incline were set to zero with shock grid settings of $25 \mathrm{~V}, 0.34 \mathrm{~mA}$, and $2 \mathrm{~Hz}$. The treadmill speed was then increased steadily to $10 \mathrm{~m} / \mathrm{min}$ (Wed) and 12 $\mathrm{m} / \mathrm{min}$ (Thurs) for an additional $10 \mathrm{~min}$.

On the Friday immediately following familiarization to the treadmill, we subjected mice to an exercise capacity test (Fig. 4B). For this, the mice were 
acclimated to the treadmill for $10 \mathrm{~min}$, with the speed and incline set initially to zero. The treadmill speed was then increased to $8.5 \mathrm{~m} / \mathrm{min}$ with an angle of inclination set to $0^{\circ}$ for $9 \mathrm{~min}$. Next, the treadmill speed and incline were increased to $10 \mathrm{~m} / \mathrm{min}$ and $5^{\circ}$, respectively, for $3 \mathrm{~min}$. The speed was then increased by 2.5 $\mathrm{m} / \mathrm{min}$ every $3 \mathrm{~min}$ to a maximum speed of $40 \mathrm{~m} / \mathrm{min}$, while inclination increased by $5^{\circ}$ every 9 min until a maximum incline of $15^{\circ}$ was achieved.

We developed strict a priori criteria for exercise-induced exhaustion. These criteria were: 1) 10 consecutive seconds on the electric grid; 2) spending more than $50 \%$ of time on the grid; and/or 3 ) lack of motivation to manual prodding. Each mouse was removed immediately from their respective lane once one or more of these criteria was reached. Following the protocol, the mice were housed separately for 30 min to avoid noticeable aggressive behavior following exercise.

Following 4 weeks of training, we repeated this testing protocol to assess changes in exercise capacity. Exercise capacity was measured using the parameters of distance run (meters achieved prior to exhaustion) and work accomplished [calculated as the product of body weight $(\mathrm{kg})$ and vertical distance $(m)$; vertical distance $=($ distance $\operatorname{run})(\sin \theta)$, where $\theta=$ the angle of inclination of the treadmill from $0^{\circ}-15^{\circ}$ ] as outlined previously. ${ }^{166}$

Exercise training. Mice assigned to exercise training groups were subjected to a 4-week protocol of forced treadmill running. The training protocol commenced the Monday after the initial exercise capacity testing with mice exercising $5 \mathrm{~d} / \mathrm{wk}$ (MonFri) at 70 or $75 \%$ of the maximal speed achieved during the initial exercise capacity 
Figure 4: Exercise testing and training design. Schematic of the familiarization, testing, and treadmill training design: (A) General treadmill training design including: familiarization, pre- and post-exercise capacity testing, and exercise training regimen; (B) Exercise capacity testing protocol; and (C) Treadmill training regimen including: warm-up and exercise training intensity and durations, and post-training housing. 
FIGURE 4

\section{A}

General Study Design:

B

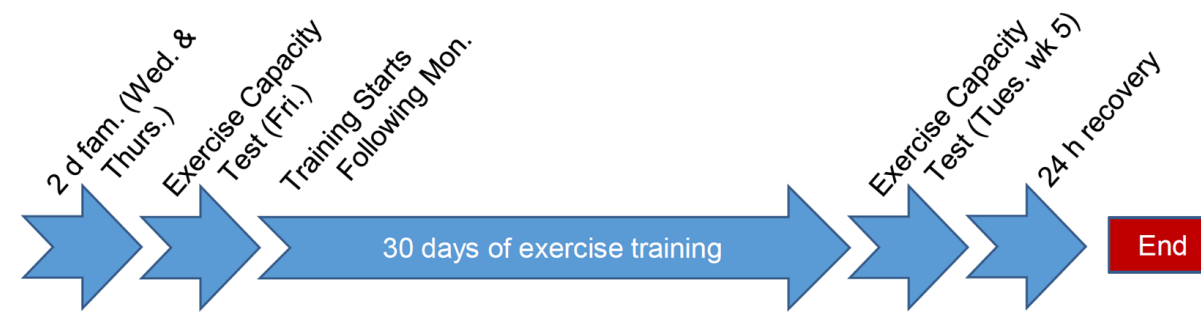

Exercise Capacity Test:

\begin{tabular}{|c|c|c|c|c|c|c|c|c|c|c|c|c|c|c|}
\hline \multirow[b]{2}{*}{$\begin{array}{l}\text { Speed } \\
(\mathrm{m} / \mathrm{min})\end{array}$} & \multirow{2}{*}{$\begin{array}{c}0^{\circ} \text { incline } \\
8.5\end{array}$} & \multicolumn{3}{|c|}{$5^{\circ}$ incline } & \multicolumn{3}{|c|}{$10^{\circ}$ incline } & \multicolumn{7}{|c|}{$15^{\circ}$ incline } \\
\hline & & 10 & 12.5 & 15 & 17.5 & 20 & 22.5 & 25 & 27.5 & 30 & 32.5 & 35 & 37.5 & 40 \\
\hline
\end{tabular}

C

Exercise Training Protocol:

\begin{tabular}{c|c|c|c|} 
& \multicolumn{3}{c}{$10^{\circ}$ incline } \\
\cline { 2 - 4 } $\begin{array}{c}\text { Speed } \\
(\mathrm{m} / \mathrm{min})\end{array}$ & 12 & 70 or $75 \%$ of maximal speed attained & $\begin{array}{c}\text { Separate } \\
\text { Housing }\end{array}$ \\
\cline { 2 - 4 } & $10 \mathrm{~min}$ & $\begin{array}{c}\text { Week } 1,40 \mathrm{~min} \text {; Week } 2,50 \mathrm{~min} ; \\
\text { Weeks } 3 \& 4,60 \mathrm{~min}\end{array}$ & $30 \mathrm{~min}$ \\
& &
\end{tabular}


test and an inclination appropriate to the speed (Fig. 4C). Prior to each training bout, we provided mice with a "warm-up" period of $10 \mathrm{~min}$ at $0 \mathrm{~m} / \mathrm{min}$ and $10 \mathrm{~min}$ at $12 \mathrm{~m} / \mathrm{min}$ to promote exercise protocol compliance and to minimize risk of injury. For strain comparison and night versus day training groups, training intensity was set at $20.8 \mathrm{~m} / \mathrm{min}, 10^{\circ}$ incline for FVB/NJ mice and $16.4 \mathrm{~m} / \mathrm{min}, 5^{\circ}$ incline for C57BL/6J mice, which corresponded to $70 \%$ of the maximal speed and the appropriate incline at the calculated speed for each strain during the initial exercise capacity test. In subsequent studies of FVB/NJ mice, mice were exercised only during the day, and in these groups, we implemented a more intensive training protocol to further examine systemic, cardiometabolic and skeletal muscle adaptations to treadmill running. For this, training intensity was set at $75 \%$ of the maximal initial exercise capacity, which corresponded to $22.3 \mathrm{~m} / \mathrm{min}$ at a $10^{\circ}$ incline. In all groups, we progressively increased the workload of the mice, such that they trained for 40 min during week 1, 50 min during week 2, and 60 min during weeks 3 and 4 . We chose this progressive intensity protocol to prevent training plateau and to stimulate systemic as well as cardiovascular and skeletal muscle adaptations. $^{166,174-176}$

Assessment of protocol compliance. To prevent injury and record protocol compliance, we monitored the mice carefully during each exercise session. Upon meeting pre-established indicators of exhaustion, mice were removed from the treadmill, and the time run was recorded (See Table 1). We used the percentage of total sessions and total minutes completed throughout the 4-week training 
program as a measure of compliance. Before and after the initial exercise capacity test, we measured blood lactate levels, which provided a biochemical indicator of exercise-induced exhaustion at or near maximal oxygen consumption $\left(\mathrm{VO}_{2} \max \right) .{ }^{177-180}$ We recorded lactate concentration in $0.7 \mu \mathrm{l}$ of blood from a small tail clip (Lactate Plus meter; Nova Biomedical) prior to the protocol and upon meeting the exhaustion criteria defined above. High lactate levels increase confidence in a successful exercise capacity test by ensuring that failure to continue is due to exhaustion at or near $\mathrm{VO}_{2} \mathrm{max}$ and not a failure to comply with the protocol. ${ }^{181-183}$

Histology. Following euthanasia, tissue was excised and rapidly fixed for immunohistochemical analysis or immediately snap frozen in liquid nitrogen and stored at $-80{ }^{\circ} \mathrm{C}$. Tissue was fixed in $10 \%$ formalin, paraffin embedded, and sectioned at $4 \mu \mathrm{m}$. Heart cross-sections were stained with 4'6-diamidino-2phenylindole (DAPI; Invitrogen) and wheat germ agglutinin (WGA; ThermoFisher) for quantification of cardiomyocyte cross-sectional area. Quantitative measurements were determined using Nikon Elements software.

Relative mitochondrial DNA measurements. Mitochondrial abundance in heart tissue was estimated by measuring mitochondrial DNA (mtDNA) abundance relative to nuclear DNA (nDNA), similar to our previous studies. ${ }^{172,184}$ Briefly, total DNA was isolated using a QIAamp DNA Mini Kit (Qiagen). A 25-mg aliquot of the tissue was homogenized, followed by overnight digestion in proteinase $\mathrm{K}$ at $55^{\circ} \mathrm{C}$. 
Following isolation, relative amounts of mtDNA and nDNA were compared using quantitative real-time PCR, using $2 \mathrm{ng}$ of the isolated DNA. Primers for cytochrome $b$ (mtDNA) and $\beta$-actin (nDNA) were used. The sequences are: cytochrome $b, 5$ 'TTGGGTTGTTTGATCCTGTTTCG-3' and

5'CTTCGCTTTCCACTTCATCTTACC-3'; and

$\beta$-actin, 5 CAGGATGCCTCTCTTGCTCT-3' and 5'-CGTCTTCCCCTCCATCGT-3'.

Statistical analysis: Unpaired or paired Student's $t$ test was used for direct comparisons; multiple groups were compared by one-way and two-way ANOVA followed by Bonferroni or Sidak Multiple Comparison test, as appropriate. For ratiobased statistical comparisons, the data were log-transformed, and unpaired Student's $t$ test was applied for assessing statistical significance between groups; we used a one-sample $t$ test for intragroup differences from a ratio of 1 (used to determine significant chronobiological differences in metabolic cage endpoints, i.e., food and water intake, physical movement, $\left.\mathrm{VO}_{2}, \mathrm{VCO}_{2}, \mathrm{RER}\right)$. A $p$ value of $\leq$ 0.05 was considered statistically significant.

\section{$\underline{\text { Results }}$}

FVB/NJ mice are not nocturnal. Laboratory mice, in general, are a nocturnal species, ${ }^{169-171}$ and they typically choose to participate in voluntary exercise at night. ${ }^{185}$ Thus, the time at which treadmill protocols are executed could be critical for ensuring exercise regimen compliance and adaptation. Therefore, we first examined the circadian characteristics of the mouse strains by measuring their 
food and water intake, locomotion, $\mathrm{VO}_{2}, \mathrm{VCO}_{2}$ and $\mathrm{RER}$ by placing untrained (naïve) mice in metabolic chambers. To assess differences between day and night behavior, a ratio (night values:day values) was calculated for each parameter. Although overall food consumption was not different between the two strains, the C57BL/6J mice consumed, on average, 3-fold more food at night. In contrast, the FVB/NJ mice showed no difference in food consumption in the night compared with the day (Fig. 5A). Cumulative water intake was not different between the strains; however, C57BL/6J mice showed significantly higher water intake at night (Fig. 5B). Similarly, C57BL/6J mice were more active at night compared with FVB/NJ mice (Fig. 5C). In general, FVB/NJ mice had fewer total beam breaks per hour $(\mathrm{C} 57 \mathrm{BL} / 6 \mathrm{~J}=2583 \pm 711 ; \mathrm{FVB} / \mathrm{NJ}=1736 \pm 582 ; p<0.01)$, indicating that cage behavioral activity is lower in this strain.

Metabolic analysis yielded analogous results. In C57BL/6J mice, oxygen consumption $\left(\mathrm{VO}_{2}\right.$; Fig. 5D), carbon dioxide production $\left(\mathrm{VCO}_{2}\right.$; Fig. 5E), and respiratory exchange ratios (RER; Fig. 5F) were higher at night compared with the day. Conversely, the FVB/NJ mice did not show metabolic differences in the day versus the night. Thus, the behavioral and metabolic qualities of C57BL/6J mice are supportive of their known nocturnal nature and are in stark contrast to FVB/NJ mice which demonstrate a relative lack of features characteristic of the nocturnal phenotype. Additionally, FVB/NJ mice had higher rates of oxygen consumption $(\mathrm{C} 57 \mathrm{BL} / 6 \mathrm{~J}=3,713 \pm 327 \mathrm{ml} / \mathrm{h} / \mathrm{kg} ; \mathrm{FVB} / \mathrm{NJ}=4058 \pm 346 \mathrm{ml} / \mathrm{h} / \mathrm{kg} ; p<0.05)$ which contributed to lower respiratory exchange ratios $(\mathrm{C} 57 \mathrm{BL} / 6 \mathrm{~J}=0.93 \pm 0.02 ; \mathrm{FVB} / \mathrm{NJ}$ $=0.86 \pm 0.02 ; p<0.0001)$. 
Figure 5: Chronobiological characteristics of C57BL/6J and FVB/NJ mice. Ratios of the average night and day values from metabolic cage analysis: (A) Food intake, (B) Water intake, (C) Total activity, (D) $\vee^{2}$, (E) $\vee \mathrm{CO}_{2}$, and (F) Respiratory exchange ratio (RER). $\mathrm{n}=10$ per group. $\left({ }^{*}\right)$ significance between C57BL/6 and FVB/NJ, $\left({ }^{\#}\right)$ significance from a ratio of $1 ;{ }^{*, \#} p<0.05,{ }^{* *}{ }^{* \#} p<0.01,{ }^{* * *},{ }^{* \#} p<0.001$. 
FIGURE 5

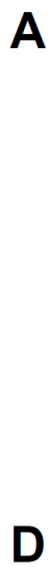

A
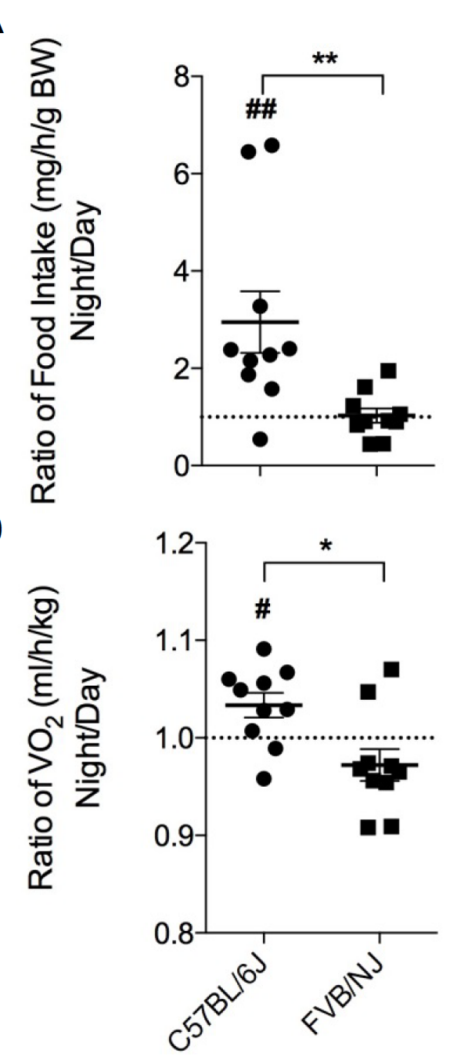

B

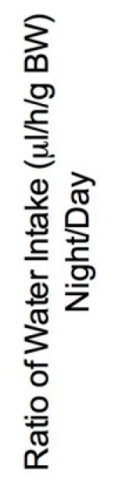

E
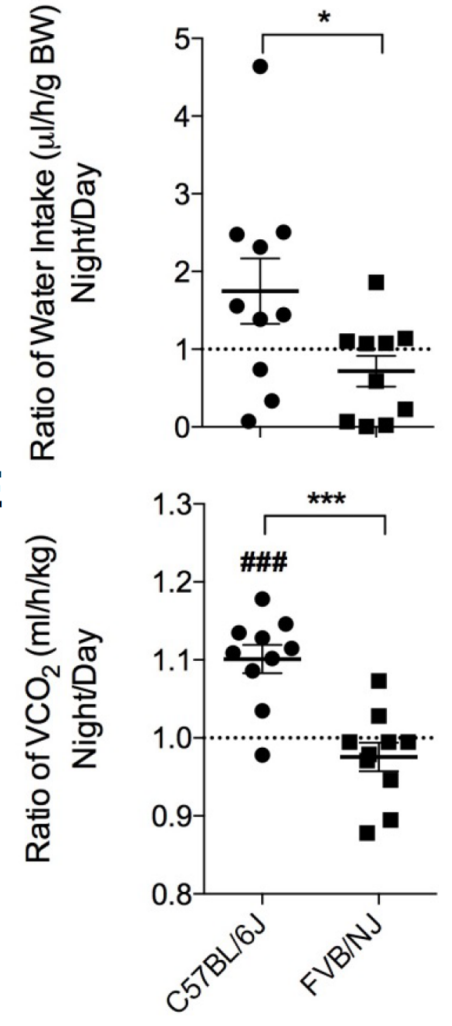

C

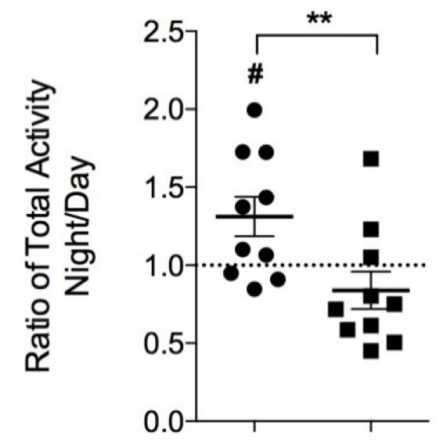

$F$

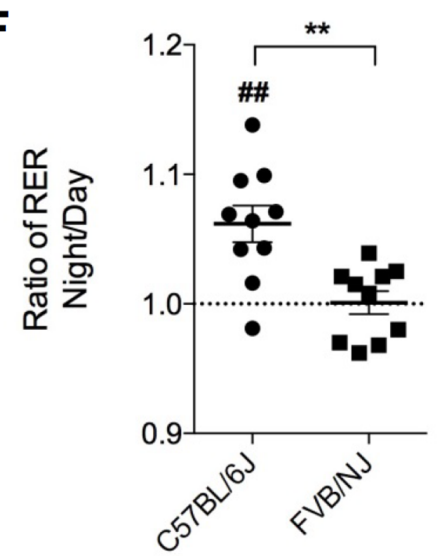


Effect of strain and time of treadmill running on initial exercise capacity. To determine the effects of mouse genetic background on initial exercise capacity, we subjected 15-week-old C57BL/6J and FVB/NJ mice to exercise capacity testing. The mice were exercised either during the day (i.e., between 9 am-12 pm) under normal laboratory lighting or at night (i.e., between 7:00 pm-10:00 pm) under dark room conditions (safelight red lamp). We chose the latter time based on metabolic cage activity data, which showed increased voluntary locomotor activity starting at 6 pm. Compared with C57BL/6J mice, the FVB/NJ strain ran 1.5-fold farther (Fig. 6A) and demonstrated $\sim 2$-fold greater initial capacity for treadmill work (Fig. 6B), regardless of when the mice were tested. Despite an apparent trend toward lower initial exercise capacity at night, we found no statistically significant differences between C57BL/6J mice exercised during the day or the night (Fig. 6B\&C); however, FVB/NJ mice showed significantly lower levels of work performed at night (Fig. 6B).

In preliminary assessments, we observed that $\mathrm{C} 57 \mathrm{BL} / 6 \mathrm{~J}$ mice received more shocks than FVB/NJ mice during treadmill exercise. Therefore, to confirm that reliable exercise capacity values were obtained and to rule out noncompliance to the testing protocol, we recorded blood lactate levels prior to and immediately following the initial exercise capacity test. Resting blood lactate values were similar in C57BL/6J and FVB/NJ mice (Fig. 6C). Although it should be noted that the night C57BL/6J group had a slightly higher resting lactate level, in general, blood lactate increased by $2-4$-fold in both strains upon meeting criteria for exhaustion (Fig. 6C); both resting and maximal lactate abundances are within the 
Figure 6: FVB/NJ mice display a higher initial exercise capacity than C57BL/6J mice. Measurements of exercise capacity and fatigue in 15-week old C57BL/6J and FVB/NJ mice tested during the day or at night: (A) Comparison of distance achieved; (B) comparison of work accomplished; (C) Blood lactate levels in C57BL/6J and FVB/NJ mice basally and following fatigue from exercise testing. $\mathrm{n}=10$ mice per group, ${ }^{*} \mathrm{p}<0.05,{ }^{* * * *} \mathrm{p}<0.0001$. 
FIGURE 6
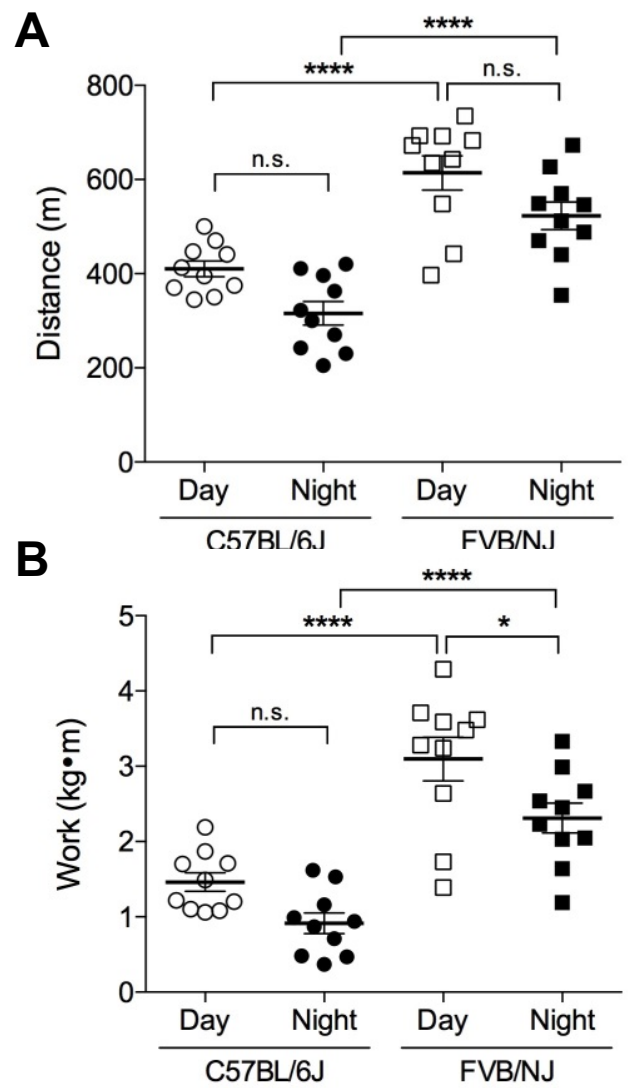

C

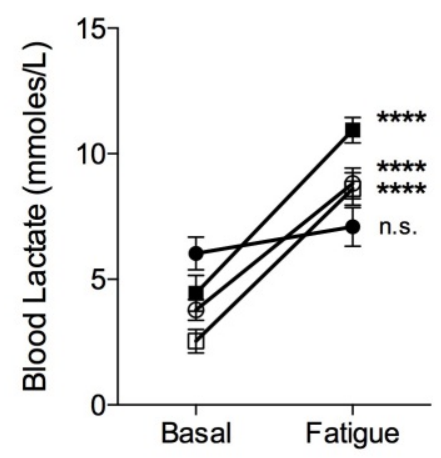


ranges previously published for resting mice and mice exercising at or near their VO2max, respectively. ${ }^{178-180}$ Collectively, these results suggest that FVB/NJ mice demonstrate a superior ability to perform treadmill work compared with C57BL/6J mice, and they have higher initial exercise capacities when tested during the day.

Effect of strain and time of training on exercise compliance and adaptation. To determine if mouse strain or the time of training (i.e., day vs. night) influences exercise protocol compliance and adaptive responses to exercise, C57BL/6J and FVB/NJ mice were subjected to a 4-week training program, with one group from each strain training at night, and one group training during the day. Each day, we recorded compliance to the training program for individual mice (Table 1). The C57BL/6J mice completed only $50-60 \%$ of their training sessions (Fig. 4A\&C), resulting in less time exercising in general (Fig. 7D). Interestingly, compliance in C57BL/6J mice diminished progressively with duration of the training protocol (Fig. 4A, Table 1). While FVB/NJ mice were generally compliant when exercised during the day or the night, $100 \%$ of the day FVB/NJ group complied with the protocol, whereas FVB/NJ mice exercising at night appeared to show modestly compromised compliance (Fig. 7B\&D); however, this did not achieve statistical significance.

Pre-training and post-training exercise capacity tests showed that, while both $\mathrm{FVB} / \mathrm{NJ}$ exercise groups significantly increased exercise capacity after four weeks of training, the C57BL/6J mice showed either no improvement or a 
Figure 7: Compliance of $\mathrm{FVB} / \mathrm{NJ}$ and $\mathrm{C} 57 \mathrm{BL} / 6 \mathrm{~J}$ to the treadmill training protocol. Compliance to a four-week treadmill training protocol in mice: $(\mathbf{A}, \mathbf{B})$ Compliance curves indicating the $\%$ of mice that completed each training session; (C) Compliance measured as the \% of total sessions; or (D) total minutes completed by mice throughout the exercise training program. $\mathrm{n}=10$ per group. * $p<0.05,{ }^{* *} p<0.001$ 
FIGURE 7

A

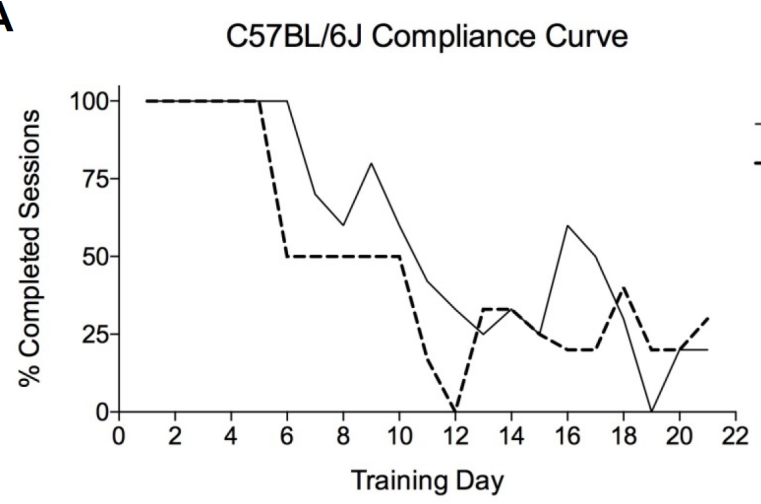

B

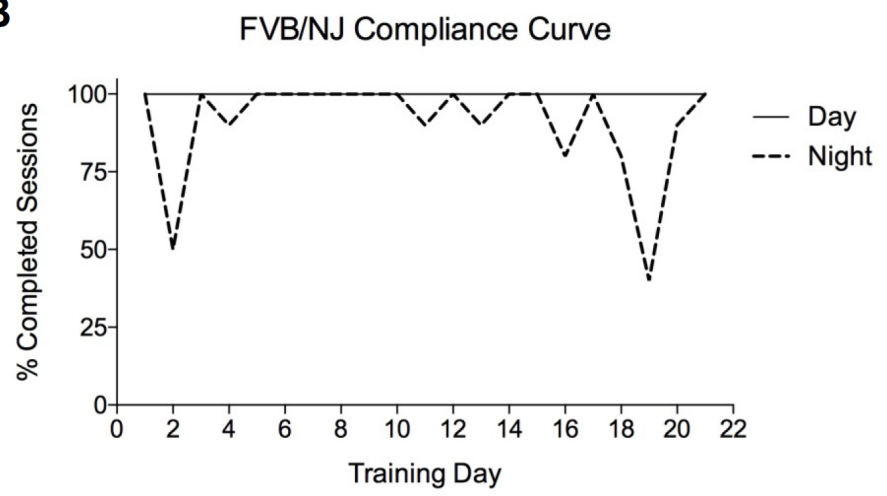

C

- Day

--. Night

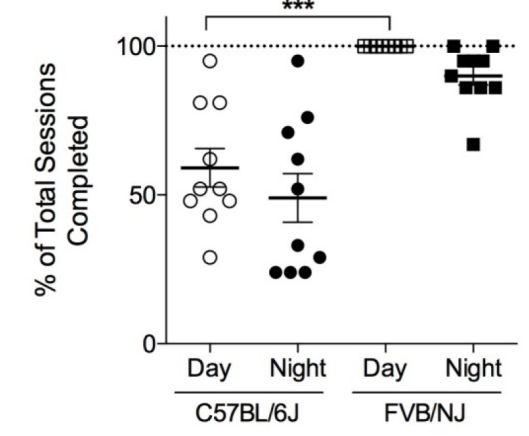

D

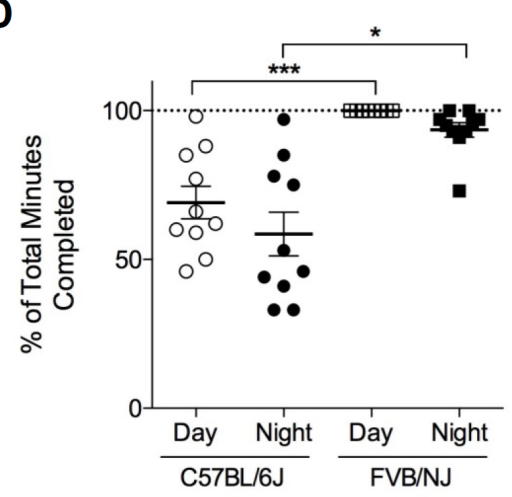


Table 1. Exercise compliance chart. Daily records of minutes completed each day during the 4 wk training program. 
TABLE 1

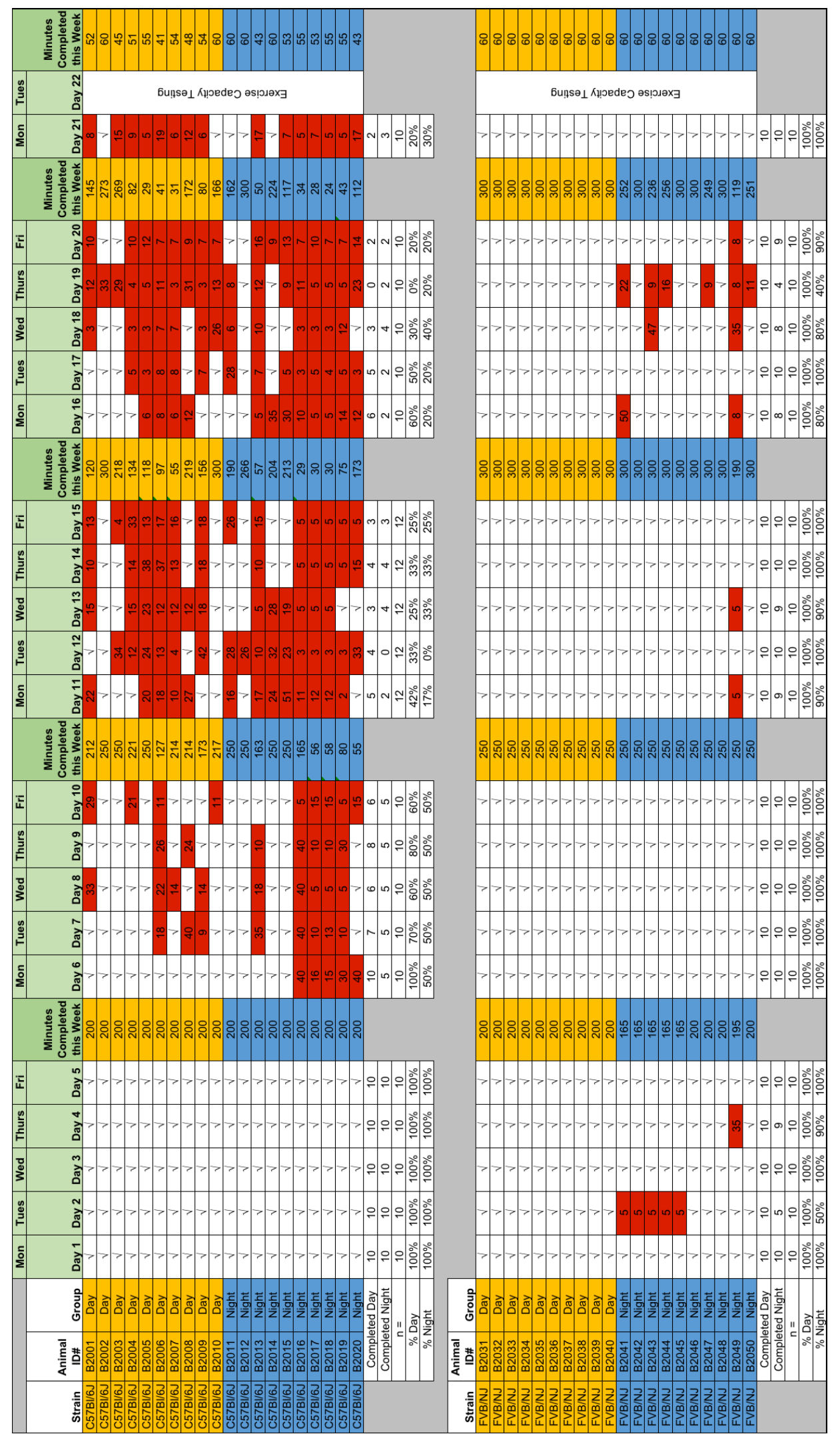


TABLE 1

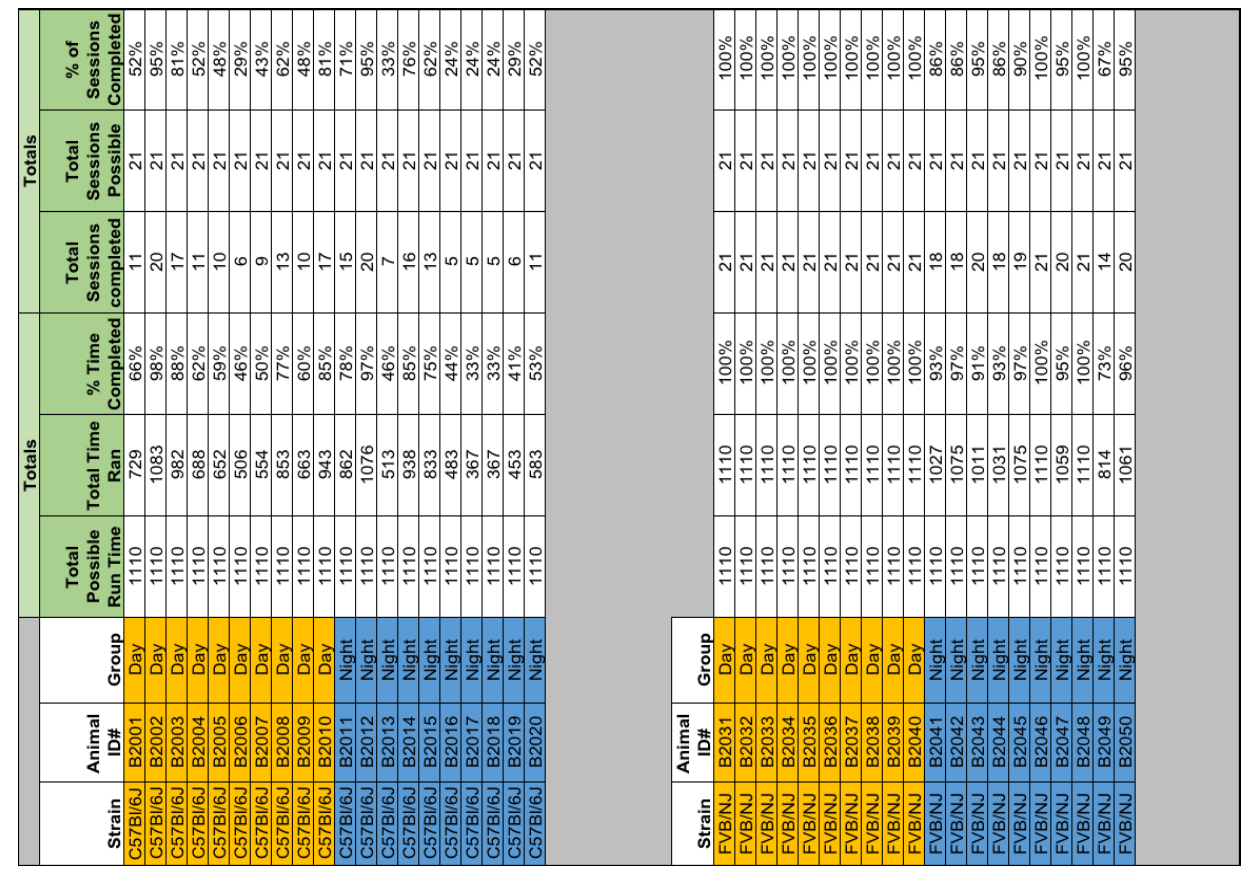


decrease in the distance run or the work accomplished following training (Fig. 8A\&B). This lack of response to exercise in the C57BL/6J mice is likely due to poor compliance to the protocol.

To determine if compliance of $\mathrm{C} 57 \mathrm{BL} / 6 \mathrm{~J}$ mice to the protocol correlated with improvements in exercise capacity, we plotted the percent improvement in distance and work against the percent time completed during the exercise regimen. As shown in Fig. 8C\&D, there is a significant, albeit weak, correlation between the percent time completed and exercise capacity in this strain. Collectively, these results show that, unlike FVB/NJ mice, C57BL/6J mice are not compliant with this extended treadmill exercise protocol, and that the time at which FVB/NJ mice exercise does not markedly influence their exercise capacity.

Physiological cardiac growth is a common endpoint used to verify cardiometabolic adaptation to exercise ${ }^{27}$ Exercise training increased heart weight to tibia length $(\mathrm{HW} / \mathrm{TL})$ in both $\mathrm{FVB} / \mathrm{NJ}$ exercise groups, whereas the $\mathrm{C} 57 \mathrm{BL} / 6 \mathrm{~J}$ mice showed no changes in heart size (Table 2). We did not find a significant correlation between exercise

time completed and HW/TL in the C57BL/6J strain (data not shown). These results indicate that exercise causes cardiac growth in FVB/NJ mice and that C57BL/6J mice, likely due to lack of compliance to this treadmill protocol, fail to demonstrate physiological cardiac growth.

FVB/NJ mice display robust adaptations following exercise training. It is known that, compared with the C57BL/6J strain, FVB/NJ mice can achieve higher critical 
Figure 8: Treadmill training-induced improvements in exercise capacity in C57BL/6J and FVB/NJ mice. Changes in exercise capacity in the mouse strains: (A) Distance run and (B) work accomplished during initial and final exercise testing following four weeks of treadmill training. $n=10$ per group. ${ }^{*} p<0.05,{ }^{* * *} p<0.001$. Panels C and D show correlations of compliance and (C) \% distance and (D) \% work improved (in the C57BL/6J strain). $\mathrm{n}=20$ mice (includes the C57BL/6J day and the C57BL/6J night groups) 
FIGURE 8

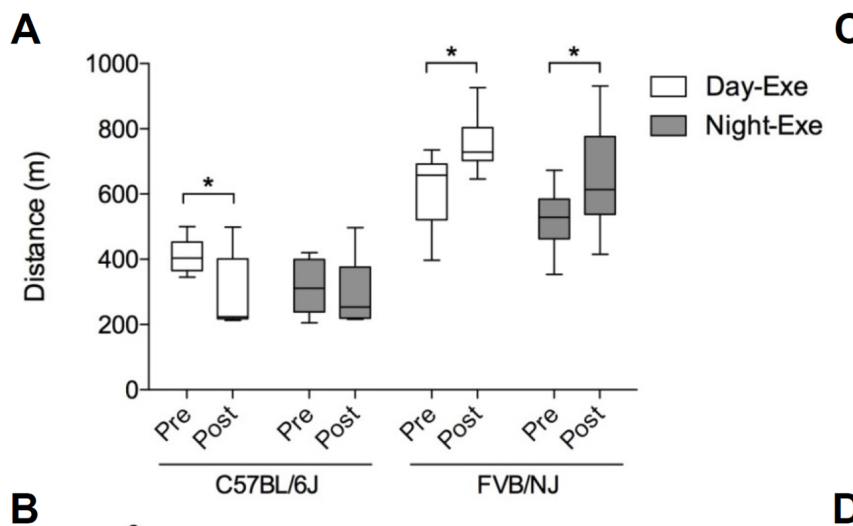

C
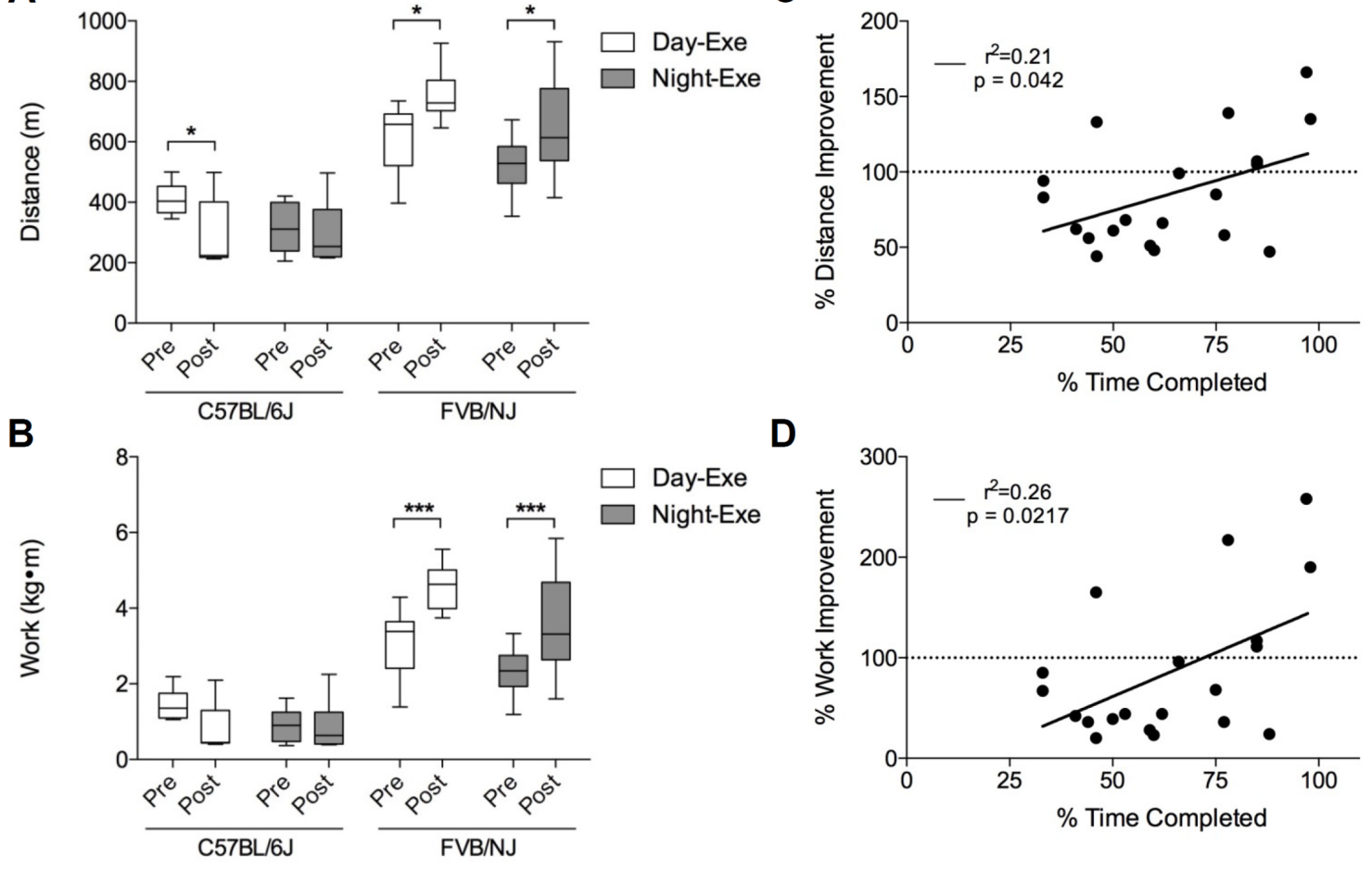

D

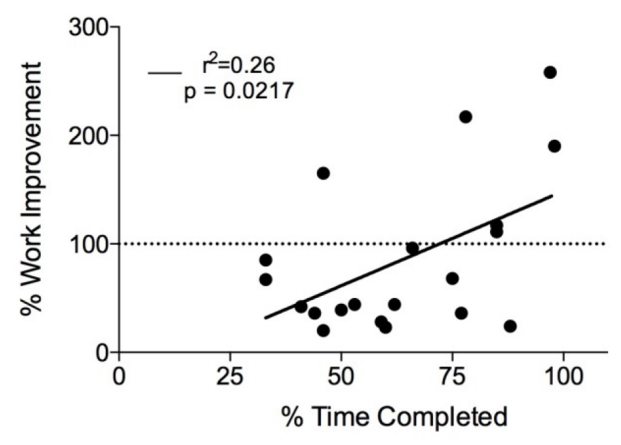


Table 2: Measurements of exercise-induced cardiac growth. Mice were trained for 4 weeks at $70 \%$ of their initial exercise capacity. Values are means \pm SD; n=10/group. HW/BW, heart weight-to-body weight ratio; HW/TL, heart weight-totibia length ratio. Statistical comparisons are between SED and EXE-Day and SED and EXE-Night. $\mathrm{n}=10$ per group. ${ }^{*} \mathrm{p}<0.05,{ }^{* * *} \mathrm{p}<0.001,{ }^{* * * *} \mathrm{p}<0.0001$. 
TABLE 2

\begin{tabular}{cll} 
& \multicolumn{1}{c}{ C57BL/6J } & \multicolumn{1}{c}{ FVB/NJ } \\
\hline Body Mass, g & & \\
SED & $28.2 \pm 2.3$ & $30.5 \pm 2.6$ \\
EXE-Day & $27.3 \pm 1.4$ & $29.9 \pm 1.7$ \\
EXE-Night & $26.4 \pm 1.8$ & $28.5 \pm 1.0$ \\
Heart Mass, mg & & \\
SED & $123.7 \pm 15.0$ & $115.4 \pm 6.1$ \\
EXE-Day & $118.7 \pm 10.1$ & $135.8 \pm 5.5^{\cdots \cdots}$ \\
EXE-Night & $118.0 \pm 13.0$ & $130.5 \pm 9.1^{\text {* }}$ \\
HW/BW, mg/g & & \\
SED & $4.4 \pm 0.4$ & $3.8 \pm 0.3$ \\
EXE-Day & $4.3 \pm 0.2$ & $4.6 \pm 0.2^{\cdots \cdots \cdots}$ \\
EXE-Night & $4.5 \pm 0.4$ & $4.6 \pm 0.2^{\cdots \cdots \cdots}$ \\
HW/TL, mg/mm & & \\
SED & $6.9 \pm 0.9$ & $6.3 \pm 0.3$ \\
EXE-Day & $6.6 \pm 0.5$ & $7.6 \pm 0.3^{\cdots \cdots}$ \\
EXE-Night & $6.6 \pm 0.7$ & $7.3 \pm 0.4^{*}$
\end{tabular}


running speeds on the treadmill, ${ }^{181}$ which indicates that their training regimen could be intensified further to evoke more robust adaptations to exercise. To test this, we trained an independent group of FVB/NJ mice at a slightly higher intensity $(75 \%$ of their initial maximum exercise capacity) for 4 weeks. Compared with pre-training exercise capacity values, this more intensive protocol yielded a $\sim 1.7$-fold improvement in distance run and a 2 2.4-fold increase in work in the mice (Fig. 9A\&B). As expected, cardiac size was significantly higher in exercised mice (Fig. 9C\&D). In exercise-adapted hearts, myocyte cross sectional area was found to be $22 \%$ higher than that found in hearts of sedentary mice, and the myocyte area distribution curves were shifted to the right (Fig. 9E-G), indicating that the exerciseinduced cardiac growth in this strain is due primarily to an increase in cardiomyocyte size. Furthermore, mitochondrial biogenesis, estimated using the ratio of relative mtDNA to nDNA content, was $53 \%$ higher in the exercise-adapted mice (Fig. 9H). These findings indicate that treadmill training in FVB/NJ mice promotes robust increases in systemic exercise capacity and elicits physiological cardiac growth. Collectively, these results indicate that treadmill exercise in FVB/NJ mice elicits robust systemic and cardiac muscle adaptations.

\section{Discussion}

The goals of this study were to examine treadmill exercise capacity, compliance, and adaptation in two commonly used strains of laboratory mice. We found that C57BL/6J mice have significantly lower treadmill exercise capacity compared with FVB/NJ mice. C57BL/6J mice, in our hands, became progressively non-compliant 
Figure 9: FVB/NJ mice display robust cardiometabolic adaptations to treadmill exercise training. Adaptations to a 4-week treadmill training program in FVB/NJ mice, where the training regimen was set based on $75 \%$ of their initial exercise capacity: (A) Distance run and (B) work accomplished during exercise testing; (C) Representative images of hearts from Sed and Exe mice; (D) HW/TL; (E) Representative cross section; $(\mathbf{F}, \mathbf{G})$ quantification of cross sectional area in heart sections stained with WGA and DAPI; and $(\mathbf{H})$ Mitochondrial biogenesis, as indicated by relative mitochondrial (mt) DNA content. $n=6-10$ mice per group. ${ }^{*} p$ $<0.05,{ }^{* *} p<0.01,{ }^{* * *} p<0.001,{ }^{* * * *} p<0.0001$. 
FIGURE 9
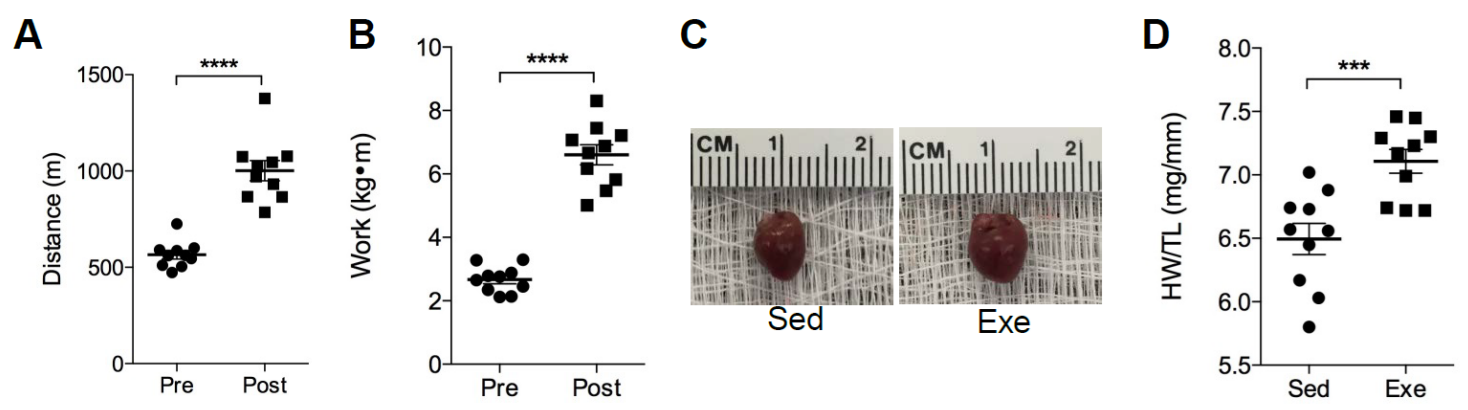

E
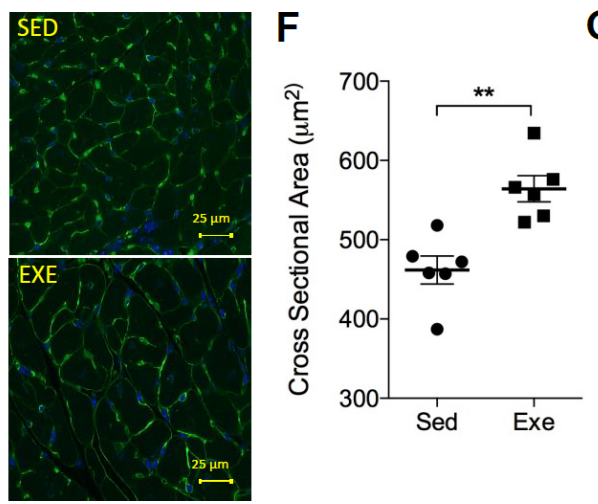

G

$\mathrm{H}$
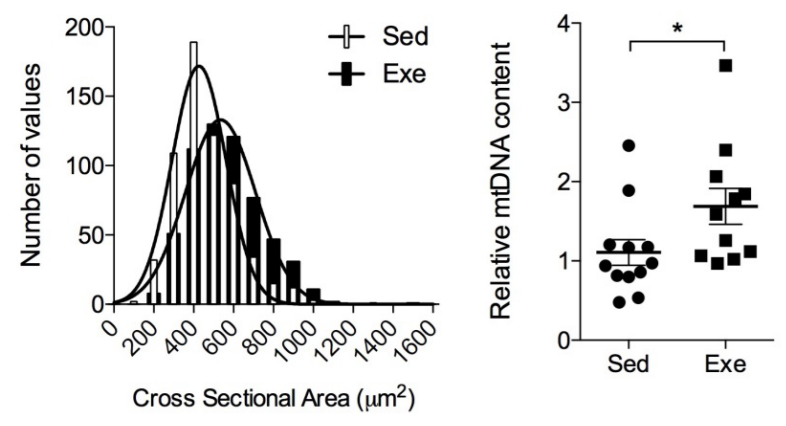
with the exercise protocol, which is a likely reason underlying their lack of adaptation to exercise. Conversely, FVB/NJ mice demonstrated near perfect compliance and showed robust increases in exercise capacity as well as cardiac adaptations. Because laboratory mice are typically nocturnal, we also compared night and day behavior in the strains as well as exercise compliance and capacity. Our metabolic cage data suggest that, contrary to the C57BL/6J strain, FVB/NJ mice have no proclivity for activity and feeding at night, and that their metabolic phenotype lacks circadian variation. Moreover, despite the nocturnal nature of C57BL/6J mice, exercising them at night did not improve compliance or exercise capacity. Collectively, these results indicate that FVB/NJ mice are a superior model for examining systemic and tissue-specific adaptation to treadmill exercise and that diurnal training appears to promote higher exercise compliance and capacity.

The increase in exercise capacity and cardiac adaptations in FVB/NJ mice are likely a consequence of their remarkable compliance as well as their superior ability to perform treadmill work. Indeed, other studies show that the FVB/NJ strain has a higher critical running speed ${ }^{181}$ and is able to run farther than C57BL/6J mice. ${ }^{163,166}$ To compare exercise capacity and adaptations between the strains in a controlled manner, we used equivalent relative intensities of training in the strains, which equated to $70 \%$ of the speed achieved during initial exercise capacity tests. These experiments showed that FVB/NJ mice could improve their exercise capacity and that exercise in this strain causes physiologic cardiac growth. Training FVB/NJ mice at a higher workload (initiated at $75 \%$ of their initial maximum speed) appeared to evoke superior improvements in distance run and 
work and provoked physiologic cardiac growth. These results indicate that the FVB strain is useful for examining intensity-dependent adaptations to exercise.

The usefulness of the treadmill model lies in its ability to control the level of exercise-associated work and to relate biochemical and physiological adaptations to workload. This is, of course, dependent on compliance to the treadmill protocol. We show that C57BL/6J mice completed the protocol during the first week of training, but that they become increasingly unwilling to run thereafter. It is unlikely that this decrease in compliance is due to overtraining because it occurred upon only the second week of training (and after a two-day rest period), and the mice did not resume their ability to exercise, even after long periods of refusing to comply with the protocol. Furthermore, the mice did not lose weight during the protocol, which can be an indicator of overtraining. ${ }^{186}$ Although it remains unclear why the C57BL/6J mice choose to run less after the first week, it could be that their tendency to receive more shocks is a negative stimulus for running, or that they associate the shocks with a positive outcome, i.e., removal from the treadmill. Interestingly, C57BL/6J mice are prone to footshock-induced analgesia, ${ }^{187,}{ }^{188}$ and they show progressively decreased shock avoidance behavior compared with other strains. ${ }^{189}$ It is also conceivable that this strain is more susceptible to negative stress in the brain caused by forced running, ${ }^{190}$ as opposed to voluntary running. Thus, it is possible that this strain acclimates to the repeated treadmill shocks that they receive during training.

Escape behavior is another potential contributing factor to the lack of compliance of C57BL/6J mice. A study by Mori and Makino showed that C57BL/6J 
mice escape and avoid shock by moving to an adjacent compartment in an L-type movement pattern rather than an R-type (rearing and jumping forward) pattern. ${ }^{191}$ This is of particular importance because the R-type pattern must be invoked to escape shock in our treadmill apparatus. Regardless, it would appear that the lack of both physiologic cardiac growth and improvements in exercise capacity in C57BL/6J mice is a result of non-compliance with the treadmill protocol.

We also show that FVB/NJ mice lack biological rhythmicity compared with C57BL/6J mice and that the time of day does not appear to affect compliance to the treadmill protocol. Lack of circadian behavior in FVB/NJ mice is a likely consequence of expression of the retinal degenerative $(r d)$ mutation, which renders them blind to visual images and appears to underlie aberrant circadian wheel running behavior and spatial awareness. ${ }^{192}$ Conversely, C57BL/6J mice appear to see well: they demonstrate entrainment to a $12: 12 \mathrm{~h}$ light:dark cycle, and they re-entrain to phase advances. ${ }^{192}$ Of note, while FVB/NJ mice are blind, C57BL/6J mice become deaf, progressively losing their hearing during the first year of life. ${ }^{193-195}$ Nevertheless, despite the impaired senses of these strains, the lack of circadian variation in the FVB/NJ strain, and the presence of a nocturnal phenotype in C57BL/6J mice, the timing at which the mice were tested and trained did not appear to have a strong impact. However, we do show that, for FVB/NJ mice, running during the day is associated with a higher initial exercise capacity. Because calculations for the 4-week training regimen are based on this initial capacity, it is possible that testing initial exercise capacities of mice during the day 
could result in a higher training workload, which could equate to increased physiological adaptations.

Although it is unclear whether the mutations that underlie loss of sight or hearing in $\mathrm{FVB} / \mathrm{NJ}$ or $\mathrm{C} 57 \mathrm{BL} / 6 \mathrm{~J}$ mice contribute to their compliance to exercise protocols, it is clear that $\mathrm{C} 57 \mathrm{BL} / 6 \mathrm{~J}$ and $\mathrm{FVB} / \mathrm{NJ}$ mice have different exercise capabilities and preferences. Consistent with previous studies, ${ }^{163,166}$ we found that C57BL/6J mice have lower treadmill exercise capacity, which did not improve even when they were tested at night. Nevertheless, several studies show that C57BL/6J mice are quite adept at voluntary wheel exercise [e.g., ${ }^{163,166,196-201}$ ], even load bearing wheels which simulate resistance training ${ }^{202}$; and, consistent with their nocturnal behavior, wheel activity in C57BL/6J mice peaks during the night. ${ }^{192}$ This suggests that the $\mathrm{C} 57 \mathrm{BL} / 6 \mathrm{~J}$ strain is more amenable to the wheel running modality than treadmill exercise. While several investigators have exercised C57BL/6J mice using a treadmill protocol successfully [reviewed in ${ }^{161}$ ], only a few studies document robust increases in exercise capacity or physiological adaptations to extended treadmill training in this strain. ${ }^{203-205}$ Most studies show adaptations that appear relatively minimal compared with other strains [e.g., 166, 206, 207] or demonstrate that additional exercise mimetics (e.g., AMPK or PPARס agonists) may be required to promote robust physiological adaptations to treadmill exercise in C57BL/6J mice. ${ }^{208}$

Several limitations of our study deserve mention. We did not measure $\mathrm{VO}_{2}$ max or identify anaerobic thresholds, which can delineate metabolic crossover points $^{209}$; however, we used stringent criteria for determining fatigue (i.e., 
behavioral criteria decided upon a priori) as well as blood lactate measurements to ensure that mice meeting these criteria were fatigued and not simply incompliant with the exercise capacity protocol. Although we show that FVB/NJ mice are proficient treadmill runners, their lack of circadian behavior suggests that they may not be suitable for investigating chronobiological changes associated with exercise (e.g., exercise-induced changes in clock genes). In addition, we did not test whether lower speeds in C57BL/6J mice or different motivating stimuli would increase compliance to the protocol. Some studies in rats, ${ }^{210}$ as well as C57BL/6J mice, ${ }^{203}$ use chocolate as a reward-based incentive to comply with the protocol. We did not test the use of reward in our study, nor did we examine whether alternative aversive stimuli (e.g., air puffs instead of shocks) would improve exercise compliance in the $\mathrm{C} 57$ strain. Thus, we do not rule out the possibility that C57BL/6J mice could be coaxed to run with an intensity and compliance similar to that of FVB/NJ mice; however, it is clear to us that improved treadmill compliance in C57BL/6J mice would appear to require a different protocol than that used here, or a reward that encourages continual compliance.

In summary, our findings indicate that FVB/NJ mice are a useful strain for testing treadmill exercise-mediated adaptations. This strain complies well with forced treadmill training and shows a robust capacity for cardiac exercise adaptation. Unlike C57BL/6J mice, FVB/NJ mice do not have a strong nocturnal nature, and they appear to show higher initial exercise capacities and comply better when trained during daytime hours. We also found that, in our hands, C57BL/6J mice show poor compliance to the treadmill exercise regimen, 
regardless of when they exercise. These findings demonstrate that FVB/NJ mice are a suitable and robust model for understanding the mechanisms underlying cardiac adaptations to exercise.

'This chapter previously appeared as an article in the journal Frontiers in Physiology. The original citation is as follows: Gibb AA, et al. FVB/NJ mice are a useful model for examining cardiometabolic adaptations to treadmill exercise. Front Physiol, 2016. 
CHAPTER III

\section{EXERCISE-INDUCED CHANGES IN GLUCOSE METABOLISM PROMOTE PHYSIOLOGIC CARDIAC GROWTH}

\section{$\underline{\text { Introduction }}$}

Exercise promotes cardiovascular wellness, ${ }^{211}$ augments musculoskeletal function, ${ }^{8}$ and lengthens lifespan. ${ }^{212}$ These salutary effects of exercise are dependent on the ability of the body to adapt to recurrent bouts of physical activity. Cumulative adaptation of the circulatory, pulmonary, and musculoskeletal systems augment exercise capacity, ${ }^{213}$ which is the strongest indicator of cardiovascular health and a robust predictor of mortality. ${ }^{214}$ Cardiovascular adaptations to exercise are particularly critical to enable adequate oxygen and nutrient delivery to peripheral tissues, and they help preserve circulatory system health with age. ${ }^{215}$, 216 Physiological cardiac growth due to exercise is a key component of these cardiovascular adaptations and contributes not only to exercise capacity, but protects the heart from injury as well. ${ }^{217}$ Nevertheless, the mechanisms by which regular exercise triggers and sustains cardiac adaptation remain unclear. Understanding how exercise promotes healthy cardiac remodeling could provide the knowledge required to delay the progression of heart disease or reverse tissue pathology. 
Previous studies have identified signaling pathways and gene programs essential for cardiac adaptation. ${ }^{27,}{ }^{218}$ For example, C/EBP $\beta$ and CITED4 are known to be critical for regulating the transcriptional programs that instigate cardiac growth $^{45,46,219}$; however, the mechanisms by which such transcriptional programs are activated with exercise are unclear. In tissues such as skeletal muscle, adaptive changes in gene expression are thought to be initiated by the repetitive bouts of metabolic stress triggered by strenuous physical activity. ${ }^{8}$ While it follows that the heart should conform to this paradigm, this has not been tested and it remains unclear which metabolic pathways and flux control steps may be important to provoke tissue adaptation to exercise.

In this study, we examined exercise-induced changes in glucose metabolism and their relationship to cardiac remodeling. We provide evidence that exercise regulates the activity of myocardial phosphofructokinase 1 (PFK1) and that the resulting changes in metabolism are sufficient to activate a transcriptional program influencing exercise-induced cardiac growth. Nonetheless, our data also indicate that chronically low or high relative levels of glycolysis, such as occurs in type II diabetes or heart failure, are damaging to mitochondria. These findings suggest that changes in myocardial glucose metabolism, brought about by regular exercise, regulate transcriptional programming and physiologic remodeling of the heart and that maintenance of metabolic flexibility is required to preserve mitochondrial health. 


\section{Experimental Procedures}

Experimental Animals. All procedures were approved by the University of Louisville Institutional Animal Care and Use Committee. The mice used in this study include transgenic mice expressing a kinase-deficient $(\mathrm{kd})$ form of PFK2 under the control of the $\alpha-M H C$ promoter (i.e., Glyco ${ }^{\text {Lo }}$ mice), transgenic mice expressing a phosphatase-deficient form of PFK2 under the control of the a-MHC promoter (i.e., Glyco $^{\mathrm{Hi}}$ mice), and wild-type (WT) littermates. ${ }^{220,}{ }^{221}$ Male mice on the FVB/NJ background were used for all studies, except for $\mathrm{F}-2,6-\mathrm{P}_{2}$ measurements in isolated cardiomyocytes, where both male and female mice were used. All studies began at 15 weeks of age; food and water were provided ad libitum; and the mice were maintained on a 12:12-h light-dark schedule, as described previously. ${ }^{63}$ At the conclusion of animal experiments, and following a $6 \mathrm{~h}$ fast, mice were anaesthetized with sodium pentobarbital (150 $\mathrm{mg} / \mathrm{kg}$, i.p.), followed by euthanasia via excision of the heart.

Exercise Capacity Testing. Mice were familiarized to the motorized rodent treadmill (Columbus Instruments, Columbus $\mathrm{OH}$ ) on the Wednesday and Thursday before the first week of training. Familiarization consisted of an initial 10 min period where the treadmill speed and incline were set to zero with shock grid settings of $25 \mathrm{~V}$, $0.34 \mathrm{~mA}$, and $2 \mathrm{~Hz}$. The treadmill speed was then increased steadily to $10 \mathrm{~m} / \mathrm{min}$ (Wed) and $12 \mathrm{~m} / \mathrm{min}$ (Thurs) for an additional $10 \mathrm{~min}$.

On the Friday immediately following treadmill familiarization, we subjected mice to an exercise capacity test. For this, the mice were acclimated to the 
treadmill for $10 \mathrm{~min}$, with the speed and incline set initially to zero. The treadmill speed was then increased to $8.5 \mathrm{~m} / \mathrm{min}$ with an angle of inclination set to $0^{\circ}$ for 9 $\min$. Next, the treadmill speed and incline were increased to $10 \mathrm{~m} / \mathrm{min}$ and $5^{\circ}$, respectively, for $3 \mathrm{~min}$. The speed was then increased by $2.5 \mathrm{~m} / \mathrm{min}$ every $3 \mathrm{~min}$ to a maximum speed of $40 \mathrm{~m} / \mathrm{min}$, while inclination increased by $5^{\circ}$ every $9 \mathrm{~min}$ until a maximum incline of $15^{\circ}$ was achieved.

A priori criteria for exercise-induced exhaustion were: 10 consecutive seconds on the electric grid; spending more than $50 \%$ of time on the grid; and/or lack of motivation to manual prodding. Each mouse was removed immediately from their respective lane once one or more of these criteria was reached. Blood lactate levels provided a biochemical indicator of exercise-induced exhaustion at or near maximal oxygen consumption $\left(\mathrm{VO}_{2} \mathrm{max}\right) .{ }^{177-180}$ We recorded lactate concentration in $0.7 \mu \mathrm{l}$ of blood from a small tail clip (Lactate Plus meter; Nova Biomedical) prior to the protocol and upon meeting the exhaustion criteria defined above. High lactate levels increase confidence in a successful exercise capacity test by ensuring that failure to continue is due to exhaustion at or near $\mathrm{VO}_{2} \mathrm{max}$ and not a failure to comply with the protocol. ${ }^{181-183}$

Following 4 weeks of training, we repeated this testing protocol to assess improvements in exercise capacity. Exercise capacity was measured using the parameters of distance run (meters achieved prior to exhaustion) and work accomplished [calculated as the product of body weight $(\mathrm{kg})$ and vertical distance $(\mathrm{m})$; vertical distance $=($ distance $\operatorname{run})(\sin \theta)$, where $\theta=$ the angle of inclination of the treadmill from $0^{\circ}-15^{\circ}$ ] as outlined previously. ${ }^{63,166}$ 
Exercise Training. Mice assigned to exercise training groups were subjected to a 4-week protocol of forced treadmill running. The training protocol commenced the Monday after the initial exercise capacity testing with mice exercising $5 \mathrm{~d} / \mathrm{wk}$ (MonFri) at $75 \%$ of their maximal speed achieved during the initial exercise capacity test and an inclination appropriate to the speed. Prior to each training bout, we provided mice with a "warm-up" period of $10 \mathrm{~min}$ at $0 \mathrm{~m} / \mathrm{min}$ and $10 \mathrm{~min}$ at $12 \mathrm{~m} / \mathrm{min}$ to promote exercise protocol compliance and to minimize risk of injury. In all groups, we progressively increased the workload of the mice, such that they trained for 40 min during week 1, 50 min during week 2 , and 60 min during weeks 3 and 4 . We chose this progressive intensity protocol to prevent training plateau and to stimulate systemic and cardiac adaptations. ${ }^{63}$

To test the effects of chronic exercise in the adapted and recovery state, mice were euthanized $24 \mathrm{~h}$ or $1 \mathrm{~h}$ after the last training session of the $4 \mathrm{wk}$ training regimen, respectively. To interrogate the effects of an acute, single-bout of exercise on cardiac metabolism, mice were subjected to familiarization as previously outlined and euthanized immediately following the completion of a $1 \mathrm{~d}$, single exercise training session; 40 min at $75 \%$ intensity. Mice were excluded from the study if they were non-compliant with the exercise protocol or if they were found to be injured or in poor health, e.g., due to fighting, etc.

Echocardiographic Assessment. Transthoracic echocardiography of the left ventricle was performed as previously described. ${ }^{222,223}$ Measurements were made 
on the Vevo 770 echocardiography instrument. Body temperature was maintained $\left(36.5-37.5^{\circ} \mathrm{C}\right)$ using a rectal thermometer interfaced with a servo-controlled heat lamp. Mice were anesthetized with $2 \%$ isoflurane, maintained under anesthesia with $1.5 \%$ isoflurane, and examined. Using short-axis B-modes and endocardial values, stroke volume (SV) was calculated as: diastolic volume - systolic volume; ejection fraction $(\mathrm{EF} \%)$ as SV/diastolic volume $\times 100 \%$; cardiac output as SV $\times$ heart rate (HR). Left ventricular diameters during diastole (LVIDd), left ventricular diameters during systole (LVIDs), and heart rate were determined from long-axis M-modes. Relative wall thickness was calculated as (diastolic posterior wall thickness + diastolic anterior wall thickness)/LVIDd. To prevent bias, the individuals performing echocardiography and image tracing were unaware of the group assignments.

Radiometric measurement of myocardial glucose utilization. Hearts were excised and perfused in non-recirculating Langendorff mode with Krebs-Henseleit $(\mathrm{KH})$ buffer $\left(37^{\circ} \mathrm{C} ; 5 \% \mathrm{CO}_{2} / 95 \% \mathrm{O}_{2}\right.$; containing in mmol/L: $118.5 \mathrm{NaCl}, 25 \mathrm{NaHCO}_{3}, 4.7$ $\mathrm{KCl}, 1.2 \mathrm{MgSO}_{4}, 1.2 \mathrm{KH}_{2} \mathrm{PO}_{4}, 2.5 \mathrm{CaCl}_{2}$ and $5 \mathrm{mM}$ glucose; $\left.\mathrm{pH} 7.4\right)$. Glycolytic flux was assessed by measuring the amount of ${ }^{3} \mathrm{H}_{2} \mathrm{O}$ released through the metabolism of exogenous $\left[5-{ }^{3} \mathrm{H}\right]$ glucose (Perkin Elmer), as previously described. ${ }^{220,221}$ Briefly, following a 20 min period of perfusion (equilibration), $\mathrm{KH}$ buffer containing 25 $\mu \mathrm{Ci} / \mathrm{ml}$ of $\left[5-{ }^{3} \mathrm{H}\right] g l u c o s e$ was infused into the cannula via a syringe infusion pump (Harvard Apparatus) at a proportion 1:100 to that of the flow rate (final concentration $=0.25 \mu \mathrm{Ci} / \mathrm{ml}$ ). The perfusate was collected in two separate 5-min 
intervals. From the collected perfusate, $400 \mu \mathrm{l}$ of perfusate was added to $25 \mu \mathrm{l}$ of $0.6 \mathrm{M} \mathrm{HCl}$ in a microcentrifuge tube. The microcentrifuge tube was then placed in a scintillation vial containing $2 \mathrm{ml}$ of $\mathrm{H}_{2} \mathrm{O}$ to allow for evaporation diffusion of $\left[{ }^{3} \mathrm{H}\right]_{2} \mathrm{O}$ in the microcentrifuge tubes into the water contained in the scintillation vial. To account for incomplete equilibration of $\left[{ }^{3} \mathrm{H}\right]_{2} \mathrm{O}$, in parallel vials, known amounts $(\mu \mathrm{Ci})$ of $\left[5^{3}{ }^{3} \mathrm{H}\right]$ glucose and $\left[{ }^{3} \mathrm{H}\right]_{2} \mathrm{O}$ (Moravek Biochemicals) were placed in microcentrifuge tubes, and placed into separate scintillation vials containing $0.5 \mathrm{ml}$ $\mathrm{dH}_{2} \mathrm{O}$. After $72 \mathrm{~h}$ incubation at $37^{\circ} \mathrm{C}$, the microcentrifuge tube was removed from the vial, $10 \mathrm{ml}$ of scintillation fluid was added, and scintillation counting was performed using a Tri-Carb 2900TR Liquid Scintillation Analyzer (Packard Bioscience Company). Glucose utilization was then calculated using the formula reported by Ashcroft, ${ }^{224}$ with considerations for: the specific activity of [5$\left.{ }^{3} \mathrm{H}\right]$ glucose; incomplete equilibration and background; the dilution of $\left[5-{ }^{3} \mathrm{H}\right]-$ to unlabeled-glucose; and scintillation counter efficiency. Glucose utilization was normalized to total heart weight and represented as a fold change to the control group. To prevent bias, the individual assisting in hanging the hearts for perfusion was unaware of the group assignments.

Measurement of blood glucose and lactate. Blood lactate concentration was measured in $0.7 \mu \mathrm{l}$ of blood from a small tail clip using a Lactate Plus meter (Nova Biomedical). Blood glucose concentration was measured from the same tail clip using the Accu-Check Aviva blood glucose monitoring meter (Roche). 
Assessment of plasma free fatty acid.: Circulating plasma free fatty acids (FFA) were assessed via a colorimetric enzymatic assay, per manufacturers guidelines (Sigma-Aldrich). Briefly, plasma was incubated with acyl-CoA synthetase for 30 min at $37^{\circ} \mathrm{C}$, in a 96 -well microplate. A master reaction mix was added to each well and samples were again incubated for $30 \mathrm{~min}$ at $37^{\circ} \mathrm{C}$. Absorbance was then measured at $570 \mathrm{~nm}$ wavelength and the concentration of FFA in each sample was calculated based on a standard curve.

Measurement of plasma IGF-1. Plasma IGF-1 was quantified using a mouse/rat IGF-1 quantikine ELISA kit (R\&D Systems, Minneapolis, MN). Briefly, plasma was diluted 500 -fold, added to microplate, and incubated for $2 \mathrm{~h}$ at room temperature with constant shaking. The microplate was then washed $5 \times$ followed by addition of secondary antibody and incubation at room temperature for $2 \mathrm{~h}$ with constant shaking. The microplate was then washed five times. For colorimetric determination, substrate solution was added to each well for $30 \mathrm{~min}$, followed by addition of stop solution and determination of color intensity by measuring at 450 $\mathrm{nm}$ with wavelength correction at $540 \mathrm{~nm}$. The concentration of IGF-1 was calculated using a standard curve.

Mitochondrial function. Heart mitochondria were isolated and subjected to respiratory function assays using the Seahorse XF24, similar to that described previously. ${ }^{225}$ Briefly, whole hearts were washed $5 \times$ with cold buffer A $(220 \mathrm{mM}$ mannitol, $70 \mathrm{mM}$ sucrose, $5 \mathrm{mM}$ MOPS, $1 \mathrm{mM}$ EDTA; $\mathrm{pH} 7.2$ with $\mathrm{KOH}$ ) followed 
by homogenization using a glass-col homogenizer in $2 \mathrm{ml}$ of buffer $\mathrm{A}$ containing $0.2 \%$ fatty acid-free BSA. Homogenate was then subjected to centrifugation at $800 \mathrm{~g}$ for $10 \mathrm{~min}$ followed by supernatant collection and centrifugation at $10,000 \mathrm{~g}$ for $10 \mathrm{~min}$. The pellet containing mitochondria was then resuspended in $1 \mathrm{ml}$ fresh buffer A (without BSA) and centrifuged at $10,000 \mathrm{~g}$, with this step repeated once. The washed mitochondrial pellet was then resuspended in $150 \mu \mathrm{l}$ respiration buffer (120 mM KCl, 25 mM Sucrose, 10 mM HEPES, 1 mM MgCl $2,5 \mathrm{mM} \mathrm{KH}_{2} \mathrm{PO}_{4}$; pH 7.2 with $\mathrm{KOH}$ ) and kept on ice.

To determine mitochondrial function, samples were diluted to a concentration of $5 \mu \mathrm{g}$ (protein) in $50 \mu \mathrm{l}$ respiration buffer per well, and centrifuged onto XF24 microplates at $500 \mathrm{~g}$ for $3 \mathrm{~min}$ at $4^{\circ} \mathrm{C}$. State 3 respiration in response to substrates were measured after injection of pyruvate + malate $(5.0 \mathrm{mM}+2.5 \mathrm{mM}$, final concentrations), succinate + rotenone $(10 \mathrm{mM}+1 \mu \mathrm{M}$, final concentrations $)$, or palmitoylcarnitine + malate $(50 \mu \mathrm{M}+0.5 \mathrm{mM}$, final concentrations $)$. The oxygen consumption rates recorded after injection of oligomycin $(1 \mu \mathrm{g} / \mathrm{ml})$, an inhibitor of ATP synthase, served as a measure of State 4 respiration. Respiratory control ratios, state $3 /$ state 4 , was calculated as a measure of the coupling of oxygen consumption to ATP production.

Measurement of F-2,6- $P_{2}$ metabolite concentration. Metabolite levels of F-2,6- $\mathrm{P}_{2}$ were measured in isolated cardiac myocytes as outlined previously. ${ }^{220,} 221$ 
Glycogen Assay. To assess myocardial glycogen content, $15 \mathrm{mg}$ of heart tissue powder was added to $100 \mu \mathrm{l}$ of ice-cold $\mathrm{dH}_{2} \mathrm{O}$, homogenized using a glass col homogenizer, boiled for $10 \mathrm{~min}$, and centrifuged at $16,000 \mathrm{~g}$ for $10 \mathrm{~min}$. Glycogen was then hydrolyzed in a 384-well plate for $1 \mathrm{~h}$ at $37^{\circ} \mathrm{C}$ followed by addition of reaction mix containing the OxiRed probe for $1 \mathrm{~h}$ at $37^{\circ} \mathrm{C}$. Absorbance was then measured at $590 \mathrm{~nm}$. The amount of glycogen was determined against a standard curve and normalized to tissue wet weight.

Histology. Following euthanasia, the heart tissue was excised, flushed with $1 \mathrm{M}$ $\mathrm{KCl}$ solution, and fixed in $10 \%$ formalin, embedded in paraffin, and sectioned at 4 $\mu \mathrm{m}$. Heart cross-sections were stained with 4'6-diamidino-2-phenylindole (DAPI; Invitrogen, Carlsbad, CA) and wheat germ agglutinin (WGA; ThermoFisher, Waltham, MA) for quantification of cardiomyocyte cross-sectional area. Additionally, sections were stained with isolectin B4 (Vector Laboratories, Burlingame, CA) to assess capillary density and sirius red for fibrosis. Quantitative measurements were determined using Nikon Elements software, with no less than 100 myocytes with centrally located nuclei assessed for area measurements. To prevent bias, the individual analyzing all histology was unaware of the group assignments. A subset of slides from each group was used based on power calculations to detect a $20 \%$ difference in cross-sectional area and capillary:myocyte ratio at $\alpha<0.05$. 
Protein and mRNA Expression Analysis. For measuring protein abundance, tissues and cells were homogenized in buffer containing $50 \mathrm{mM} \mathrm{HEPES} \mathrm{(pH} \mathrm{7.0),}$ $2 \mathrm{mM}$ EDTA, $1 \% \mathrm{NP}-40,10 \%$ glycerol, $1 \mathrm{mM} \mathrm{MgCl} 2,1 \mathrm{mM} \mathrm{CaCl}$, and $150 \mathrm{mM}$ $\mathrm{NaCl}$; fresh phosphatase and protease inhibitors were added immediately prior to tissue homogenization. The homogenates were sonicated on ice and centrifuged at $13,000 \mathrm{~g}$ for $20 \mathrm{~min}$. Proteins in the supernatant were separated by SDS-PAGE, electroblotted to PVDF membranes, and probed according to the manufacturers' protocols. A horseradish peroxidase-linked secondary antibody (Cell Signaling Technology, Danvers, MA) was used to detect immunoreactive proteins. Images were recorded using a Fujifilm LAS-300 imager, and relative protein abundance was measured via densitometric analysis using TotalLab software.

For transcriptional expression analysis via RT-PCR, RNA was extracted from tissues and cells using the RNeasy Plus Universal Kit (Qiagen, Germantown, MD), followed by cDNA synthesis. Real-time PCR amplification was performed with SYBR Green quantitative PCR (qPCR) Master Mix (SA Biosciences, Germantown, MD) using a 7900HT Fast Real-Time PCR System (Applied Biosystems, Foster, CA). Relative expression was determined by the $2^{-\Delta \Delta C t}$ method. A list of the antibodies and primers used can be found below.

\section{Antibodies used in these studies.}

\begin{tabular}{|l|l|l|}
\hline Antibodies & Source & Identifier \\
\hline $\begin{array}{l}\text { DAPI (4',6-Diamidino-2- } \\
\text { Phenylindole, Dihydrochloride) }\end{array}$ & ThermoFisher & Cat\#D1306 \\
\hline $\begin{array}{l}\text { WGA (Wheat germ agglutinin, } \\
\text { Alexa Fluor 647 conjugate) }\end{array}$ & ThermoFisher & Cat\#W32466 \\
\hline $\begin{array}{l}\text { Fluorescein labeled griffonia } \\
\text { simplicifolia lectin I (GSL I) } \\
\text { isolectin B4 }\end{array}$ & Vector Laboratories & Cat\#FL-1201 \\
\hline
\end{tabular}




\begin{tabular}{|l|l|l|}
\hline Sirius Red & Sigma-Aldrich & Cat\#365548 \\
\hline $\begin{array}{l}\text { Rabbit monoclonal anti-p-Akt } \\
\text { (S473) }\end{array}$ & Cell Signaling Technology & Cat\#4060 \\
\hline Rabbit polyclonal anti-Akt & Cell Signaling Technology & Cat\#9272 \\
\hline $\begin{array}{l}\text { Rabbit monoclonal anti-p-PFK2 } \\
\text { (S483;PFKFB2) }\end{array}$ & Cell Signaling Technology & Cat\#13064 \\
\hline $\begin{array}{l}\text { Rabbit polyclonal anti-PFK2 } \\
\text { (PFKFB2; heart isoform) }\end{array}$ & Bethyl Labs & Cat\#A304-286A-M \\
\hline Rabbit monoclonal anti-HK1 & Cell Signaling Technology & Cat\#2024 \\
\hline Rabbit monoclonal anti-HK2 & Cell Signaling Technology & Cat\#2867 \\
\hline Rabbit polyclonal anti-PFK1 & Santa Cruz Biotechnology & Cat\#sc-67028 \\
\hline $\begin{array}{l}\text { Rabbit polyclonal anti-PFK2 } \\
\text { (PFKFB1; liver isoform) }\end{array}$ & Novus Biologicals & Cat\#NB100-92391 \\
\hline $\begin{array}{l}\text { Rabbit polyclonal anti-PFK2 } \\
\text { (PFKFB3; ubiquitous isoform) }\end{array}$ & Proteintech & Cat\#13763-1-AP \\
\hline Rabbit monoclonal anti-PKM1/2 & Cell Signaling Technology & Cat\#3106 \\
\hline Rabbit polyclonal anti-GFAT1 & Santa Cruz Biotechnology & Cat\#sc-134894 \\
\hline Rabbit polyclonal anti-GS & Cell Signaling Technology & Cat\#3893 \\
\hline Mouse monoclonal anti-LDHB & Abcam & Cat\#ab85319 \\
\hline
\end{tabular}

\section{PCR primers used in these studies.}

\begin{tabular}{|l|l|l|}
\hline \multicolumn{2}{|l|}{ Sequence-Based Reagents } \\
\hline $\begin{array}{l}\text { Gene of } \\
\text { Interest }\end{array}$ & Forward & Reverse \\
\hline Cited4 & CATGGACACCGAGCTCATC & CTGACCCCAGGTCTGAGAAG \\
\hline Cebpb & GGGGTTGTTGATGTTTTGGT & TCGAAACGGAAAAGGTTCTCA \\
\hline Nfatc2 & CACAGATACGGTGACCCCT & GTGGGATCGGGTTCTTCTTC \\
\hline HPRT & AGGACCTCTCGAAGTGTTGG & AGGGCATATCCAACAACAAAC \\
\hline
\end{tabular}

Analysis of cardiac ultrastructure. Transmission electron microscopy analysis, left ventricular tissue was dissected into $3 \mathrm{~mm}^{3}$ pieces and fixed in $2.5 \%$ glutaraldehyde overnight. The samples were then post-fixed in $2 \%$ aqueous osmium tetroxide, dehydrated in ascending concentrations of ethanol and embedded in Araldite 502 (Electron Microscopy Sciences, Hatfield PA). Onemicron thick sections were stained with toluidine blue for screening under a light 
microscope. Selected blocks in which the muscle had a longitudinal orientation were cut into $800 \AA$ thick sections, mounted on 200-mesh copper grids, doubly stained with uranyl acetate and lead citrate, and examined in a Phillips CM-10 electron microscope with a LaB 6 cathode (Phillips Electronic Instruments Co., New York, NY) operating at $80 \mathrm{kV}$.

Metabolomic Analysis. Hearts were freeze-clamped in situ using liquid nitrogencooled Wollenberger Tongs, which helps to capture the metabolome as it is in the beating mouse heart in vivo. Samples were prepared by Metabolon using their automated MicroLab STAR ${ }^{\bullet}$ system (Hamilton Company, Reno, NV). First, tissue homogenates were made in water at a ratio of $5 \mathrm{uL}$ per $\mathrm{mg}$ of tissue. For quality control, several recovery standards were added prior to the first step in the extraction process. To remove protein, dissociate small molecules bound to protein or trapped in the precipitated protein matrix, and to recover chemically diverse metabolites, proteins were then precipitated with methanol (final concentration $80 \% \mathrm{v} / \mathrm{v}$ ) under vigorous shaking for 2 min (Glen Mills GenoGrinder 2000) followed by centrifugation. For quality assurance and control, a pooled matrix sample was generated by taking a small volume of each experimental sample to serve as a technical replicate throughout the data set. Extracted water samples served as process blanks. A cocktail of standards known not to interfere with the measurement of endogenous compounds was spiked into every analyzed sample, allowing instrument performance monitoring and aiding chromatographic alignment. 
The extract was divided into fractions for analysis by reverse phase (RP)/UPLC-MS/MS with positive ion mode electrospray ionization (ESI), by RP/UPLC-MS/MS with negative ion mode ESI, and by HILIC/UPLC-MS/MS with negative ion mode ESI. Samples were placed briefly on a TurboVap ${ }^{\circledR}$ (Zymark) to remove the organic solvent. All methods utilized a Waters ACQUITY UPLC and a Thermo Scientific Q-Exactive high resolution/accurate mass spectrometer interfaced with a heated electrospray ionization (HESI-II) source and Orbitrap mass analyzer operated at 35,000 mass resolution. The sample extract was reconstituted in solvents compatible with each MS/MS method. Each reconstitution solvent contained a series of standards at fixed concentrations to ensure injection and chromatographic consistency. One aliquot was analyzed using acidic positive ion conditions, chromatographically optimized for hydrophilic compounds. In this method, the extract was gradient eluted from a C18 column (Waters UPLC BEH C18-2.1 x100 mm, $1.7 \mu \mathrm{m}$ ) using water and methanol, containing $0.05 \%$ perfluoropentanoic acid (PFPA) and $0.1 \%$ formic acid (FA). For more hydrophobic compounds, the extract was gradient eluted from the aforementioned C18 column using methanol, acetonitrile, water, $0.05 \%$ PFPA and $0.01 \%$ FA. Aliquots analyzed using basic negative ion optimized conditions were gradient eluted from a separate column using methanol and water, containing $6.5 \mathrm{mM}$ ammonium bicarbonate $(\mathrm{pH}$ 8). The last aliquot was analyzed via negative ionization following elution from a HILIC column (Waters UPLC BEH Amide $2.1 \times 150 \mathrm{~mm}, 1.7 \mu \mathrm{m}$ ) using a gradient consisting of water and acetonitrile with $10 \mathrm{mM}$ ammonium formate $(\mathrm{pH}$ 10.8). The 
MS analysis alternated between MS and data-dependent $\mathrm{MS}^{n}$ scans using dynamic exclusion. The scan range covered $70-1000 \mathrm{~m} / \mathrm{z}$.

Raw data were extracted, peak-identified and processed using Metabolon's proprietary hardware and software. Compounds were identified by comparison to library entries of purified, authenticated standards or recurrent unknown entities, with known retention times/indices (RI), mass to charge ratios $(\mathrm{m} / \mathrm{z})$, and chromatographic signatures (including MS/MS spectral data). Biochemical identifications were based on three criteria: retention index within a narrow RI window of the proposed identification, accurate mass match to the library $\pm 10 \mathrm{ppm}$, and the MS/MS forward and reverse scores between experimental data and authentic standards. Proprietary visualization and interpretation software (Metabolon, Inc., Durham, NC) was used to confirm the consistency of peak identification among the various samples. Library matches for each compound were checked for each sample and corrected, if necessary. Area under the curve was used for peak quantification.

Original scale data (raw area counts) were analyzed using Metaboanalyst 3.0 software (http://www.metaboanalyst.ca/). ${ }^{226}$ Metabolites with greater than $50 \%$ of the values missing were omitted from the analysis, and missing values were imputed by introducing values with half the minimum positive value in the original data. An interquartile range filter was used to identify and remove variables unlikely to be of use when modeling the data. The data were log-transformed and auto-scaled (mean-centered and divided by the standard deviation of each variable). Univariate (e.g., volcano plots) and multivariate (e.g., PCA) analyses 
were then performed. For multiple comparison testing, $q$ values were calculated as in R using a method embedded within the Metaboanalyst software, controlling for the false discovery rate. ${ }^{227}$ Radar charts were constructed in $\mathrm{R}$ using average Z-scores of significantly different metabolites from each biochemical pathway.

Transcriptomic analyses. Hearts of male WT, Glyco ${ }^{\mathrm{LO}}$ and Glyco ${ }^{\mathrm{Hi}}$ mice (15-16 weeks of age) were subjected to transcriptomic analysis using the GeneChip ${ }^{\circledR}$ Mouse Genome 430 2.0 Array (Affymetrix, Inc.). Array data were analyzed using the affy ${ }^{228}$ and limma ${ }^{229}$ packages of $R$ for Robust Multi-array Average (RMA) for normalization and for moderate t-statistics to derive $p$-values, respectively. The $p$ values were adjusted using the Bejamini and Hochberg method for false discovery rate control. ${ }^{227}$ For the genes with multiple probe set IDs, the probe set with the highest standard deviation among all samples was kept for further analsyis. Gene Ontology analysis was performed using DAVID (https://david.ncifcrf.gov/home.jsp. ${ }^{230}$

Data and Software Availability. Cytoscape software was used to visualize changes in metabolic pathways and networks and was facilitated using the MetaboLync Cytoscape Plugin (Metabolon, Inc). Metabolomic data were analyzed using Metaboanalyst 3.0 software. Gene array data has been deposited in the Gene Expression Omnibus databank (GSE100176) and will be made available to readers upon publication. 
Statistical analysis. Statistical parameters including the value of $\mathrm{n}$ (number of mice), the definition of center, dispersion and precision measures (mean \pm SEM or SD), and statistical significance is reported in the Figures and Figure Legends. A $p$ value of $\leq 0.05$ was considered statistically significant. In the Figures, asterisks denote statistical significance as calculated by unpaired or paired Student's $t$ test (for direct comparisons); Levene's test was used to assess the equality of group variances between groups, and if found to be significantly different, a Welchmodified t-test was used to assess significance. Multiple comparisons were assessed by one-way or two-way ANOVA followed by Bonferroni or Sidak Multiple Comparison tests $\left({ }^{*}, p<0.05 ;{ }^{* *}, p<0.01 ;{ }^{* * *}, p<0.001 ;{ }^{* * *}, p<0.0001\right)$, as appropriate. Statistical analysis was performed using GraphPad Prism 7, Metaboanalyst, and/or the R program.

\section{$\underline{\text { Results }}$}

Exercise promotes physiologic cardiac growth. Wild-type mice subjected to 4 weeks of treadmill training showed a $48 \%$ increase in distance run and a $77 \%$ increase in work accomplished (Fig. 10A, B); successful exercise testing was validated by elevated blood lactate levels, indicating that the mice ran to exhaustion (Fig. 10C). In comparison with untrained mice, trained mice had 15\% higher levels of circulating plasma IGF-1 (Fig. 10D), further demonstrating systemic adaptation to the training regimen. Exercise-adapted mice displayed physiologic cardiac growth characterized by a $10 \%$ increase in heart weight to tibia 
Figure 10: Exercise training improves exercise capacity. Systemic adaptation to a 4-wk treadmill training protocol: Pre- and post-training $(\mathbf{A})$ distance and $(\mathbf{B})$ work; (C) Validation of a successful high-intensity exercise capacity test as indicated by levels of blood lactate measured at rest and immediately after the criteria for exercise-induced fatigue were met; and (D) Plasma levels of insulin-like growth factor-1 (IGF-1). Sed, sedentary; Exe, exercise. Data represented as mean \pm SEM; ${ }^{* * *} p<0.001,{ }^{* * * *} p<0.0001 ; n=6$ per group. 
FIGURE 10

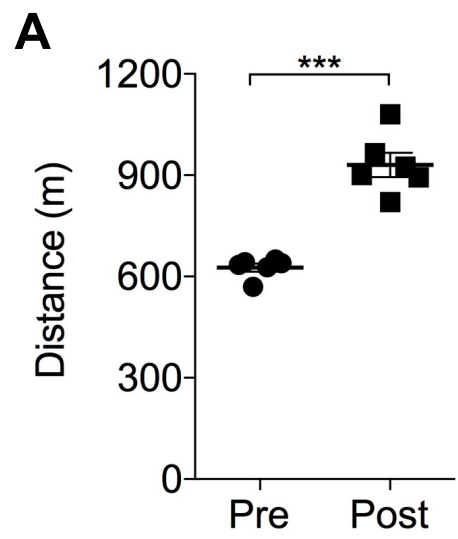

B
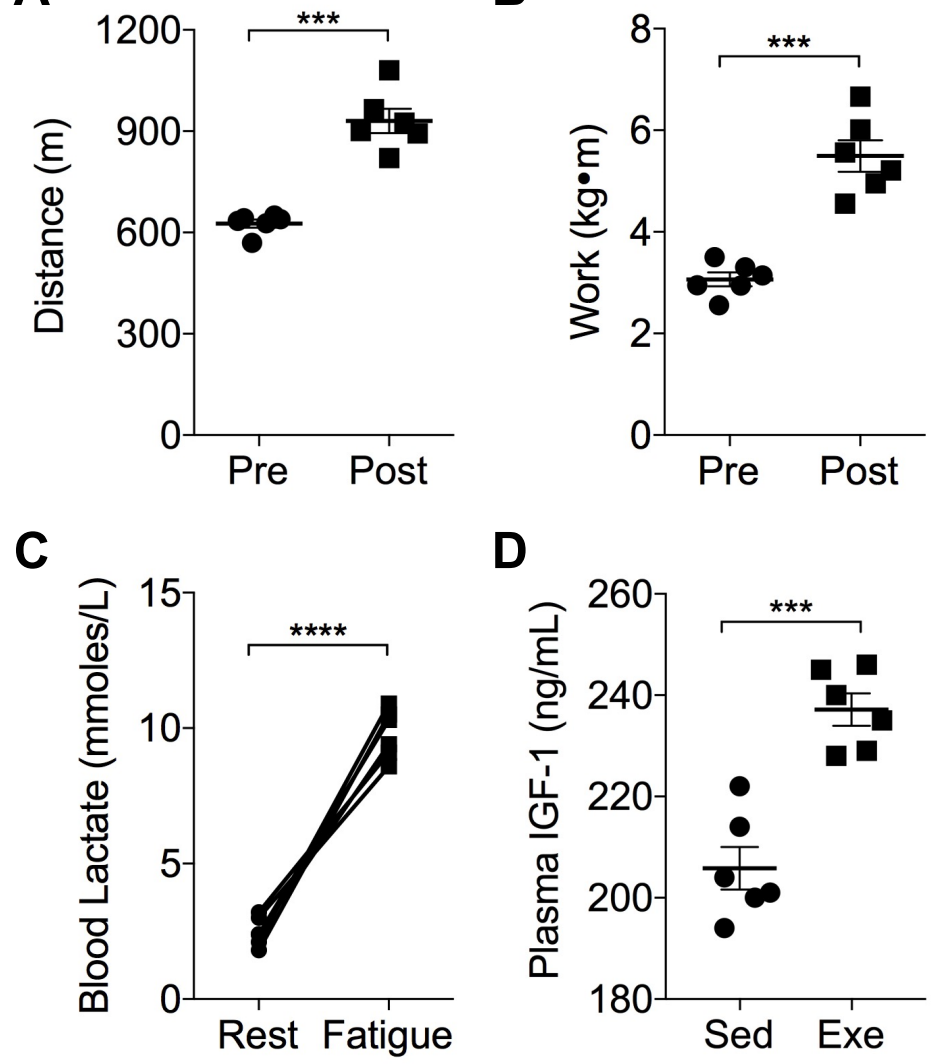

D

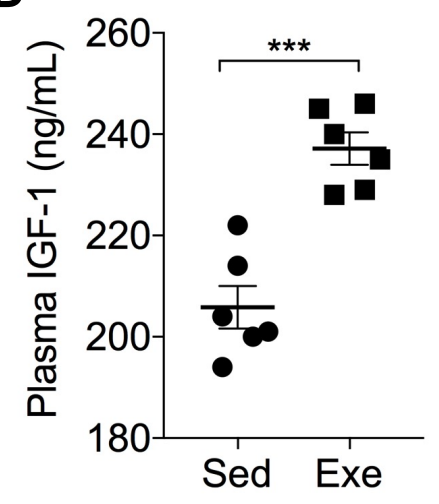


Figure 11: Exercise training promotes physiologic cardiac growth. Effect of treadmill training on the exercise-induced cardiac growth program: (A) Gravimetric measurements of cardiac size (HW/TL, heart weight/tibia length); (B) Representative myocardial sections stained with wheat germ agglutinin (red) and isolectin B4 (green). Nuclei are stained with DAPI (blue); (C) Cardiomyocyte crosssectional area; (D) Capillary-to-myocyte ratio; (E) Phosphorylation of AKT (Ser473) $24 \mathrm{~h}$ following the last training session; (F) Relative Mrna expression of Cebpb, Cited4, and Nfatc2. Data are represented as mean \pm SEM; ${ }^{*} p<0.05,{ }^{* *} p<0.01$, ${ }^{* * *} p<0.001 ; n=5-6$ per group. 
FIGURE 11

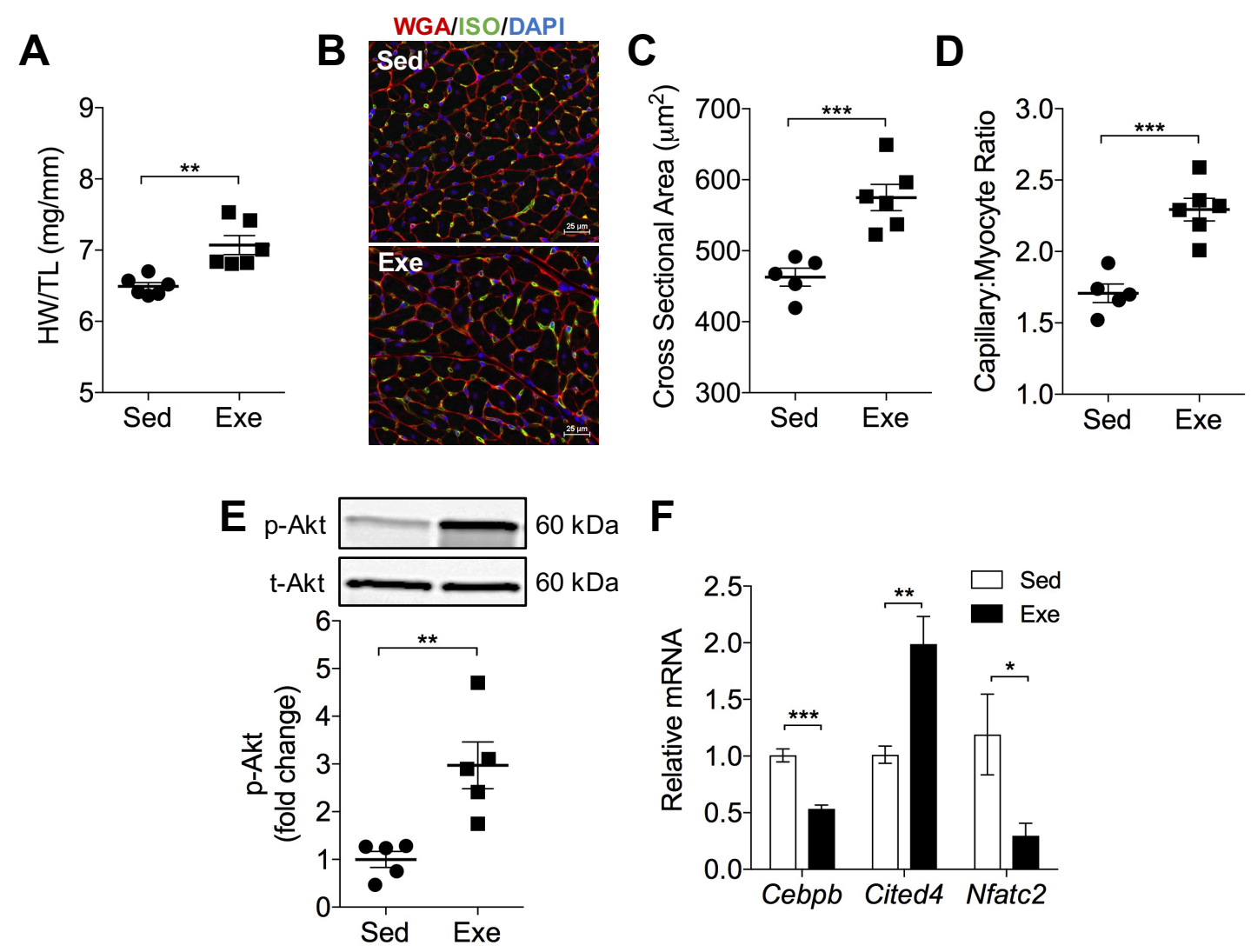


Table 3: Echocardiographic data from sedentary and exercised WT mice. After acclimatization and an initial exercise capacity test, male FVB/NJ mice (15 weeks of age) were subjected to exercise training $5 \mathrm{~d}$ a week for 4 wk. One day following the final exercise capacity test, the mice were anesthetized and cardiac structure and function were assessed by echocardiography. Abbreviations: Sed, sedentary; Exe, exercised; HR, heart rate; EDV, end-diastolic volume; ESV, endsystolic volume; SV, stroke volume; EF, ejection fraction; CO, cardiac output; LVIDd, left ventricular internal diameter in diastole; LVIDs, left ventricular internal diameter in systole; LVPWd, left ventricular posterior wall in diastole; LVPWs, left ventricular posterior wall in systole; LVAWd, left ventricular anterior wall in diastole; LVAWs, left ventricular anterior wall in systole; RWT, relative wall thickness. ${ }^{*} p<0.05,{ }^{* *} p<0.01$ vs. Sed. 
TABLE 3

\begin{tabular}{|r|r|l|l|r|l|l|}
\hline & \multicolumn{3}{|c|}{ WT-Sed } & \multicolumn{3}{c|}{ WT-Exe } \\
\hline & Mean & & SD & Mean & & SD \\
\hline HR (bpm) & 482 & \pm & 32 & 492 & \pm & 25 \\
\hline Endocardial Values & & & & & & \\
\hline EDV $(\mu \mathrm{l})$ & 38 & \pm & 3 & 45 & \pm & $6^{* *}$ \\
\hline ESV $(\mu \mathrm{l})$ & 11 & \pm & 3 & 13 & \pm & 2 \\
\hline SV $(\mu \mathrm{l})$ & 27 & \pm & 3 & 32 & \pm & $5^{* *}$ \\
\hline EF (\%) & 71 & \pm & 7 & 72 & \pm & 4 \\
\hline CO (ml/min) & 13 & \pm & 2 & 16 & \pm & $2^{* *}$ \\
\hline Chamber Diameter & & & & & & \\
\hline LVIDd (mm) & 3.7 & \pm & 0.2 & 4.0 & \pm & $0.2^{* *}$ \\
\hline LVIDs (mm) & 2.2 & \pm & 0.2 & 2.5 & \pm & $0.2^{* *}$ \\
\hline Wall Thickness & & & & & & \\
\hline LVPWd (mm) & 0.8 & \pm & 0.1 & 0.9 & \pm & $0.1^{*}$ \\
\hline LVPWs (mm) & 1.2 & \pm & 0.1 & 1.3 & \pm & $0.1^{*}$ \\
\hline LVAWd (mm) & 0.8 & \pm & 0.1 & 0.8 & \pm & 0.1 \\
\hline LVAWs (mm) & 1.1 & \pm & 0.2 & 1.1 & \pm & 0.2 \\
\hline RWT & 0.46 & \pm & 0.07 & 0.42 & \pm & 0.07 \\
\hline $\mathrm{n}$ & \multicolumn{3}{|c|}{12} & \multicolumn{3}{|c|}{10} \\
\hline
\end{tabular}


length (HW/TL; Fig. 11A), a 24\% increase in cardiomyocyte cross-sectional area, and a $35 \%$ increase in capillary-to-myocyte ratio (Fig. 11B-D). Furthermore, exercise increased chamber diameter and wall thicknesses, improved indices of cardiac function (EDV, SV, and CO; Table 3), and activated the pro-hypertrophic signaling intermediate protein kinase $B(A K T)^{35,39-42}$ (Fig. 11E). Exercise training downregulated Cebpb and upregulated Cited4, an observation which is consistent with previous findings showing necessary involvement of these transcriptional mediators in regulating physiologic growth. ${ }^{45,46,217-219}$ Moreover, the expression of $\mathrm{Nfatc2}$, the deletion of which ameliorates pathological hypertrophy and heart failure but does not prevent physiological hypertrophy, ${ }^{231}$ was markedly lower in hearts from exercise-adapted mice (Fig. 11F). Collectively, these results indicate that exercise training elicits robust cardiac adaptation in mice.

Metabolomic changes in the exercise-adapted heart at steady state. To examine global metabolic changes in exercise-adapted hearts, we first compared the relative abundance of 424 metabolites in hearts isolated from sedentary and exercise-adapted mice. To capture the metabolic state of the adapted heart as accurately as possible, the hearts were freeze-clamped in situ $24 \mathrm{~h}$ after the final exercise bout (Fig. 12A). Principal component analysis (PCA) showed little separation between the groups (Fig. 12B), and only 20 metabolites (out of 424 measured) were found to be significantly different (Fig. 12C). Of these, only two metabolites had a $q$ value below 0.05 (Table 4). These findings demonstrate that 
Figure 12: Metabolomic changes in the exercise-adapted mouse heart. (A) Mice were either exercised for 4-wk (EXE) or remained sedentary (SED) under similar handling conditions. Following a $6 \mathrm{~h}$ fast and $24 \mathrm{~h}$ after the final exercise session, hearts were freeze-clamped in situ. The relative abundance of metabolites in the hearts was examined by LC/MS/MS; (B) 2D PCA analysis; (C) Volcano plot showing metabolites that changed $\geq 1.25$-fold $(p \leq 0.05)$ in Exe hearts compared with Sed hearts. A list of metabolites found to be significantly different in exercise-adapted hearts can be found in Table S2. $\mathrm{N}=9$ Sed and 11 Exe mouse hearts per group. 
FIGURE 12
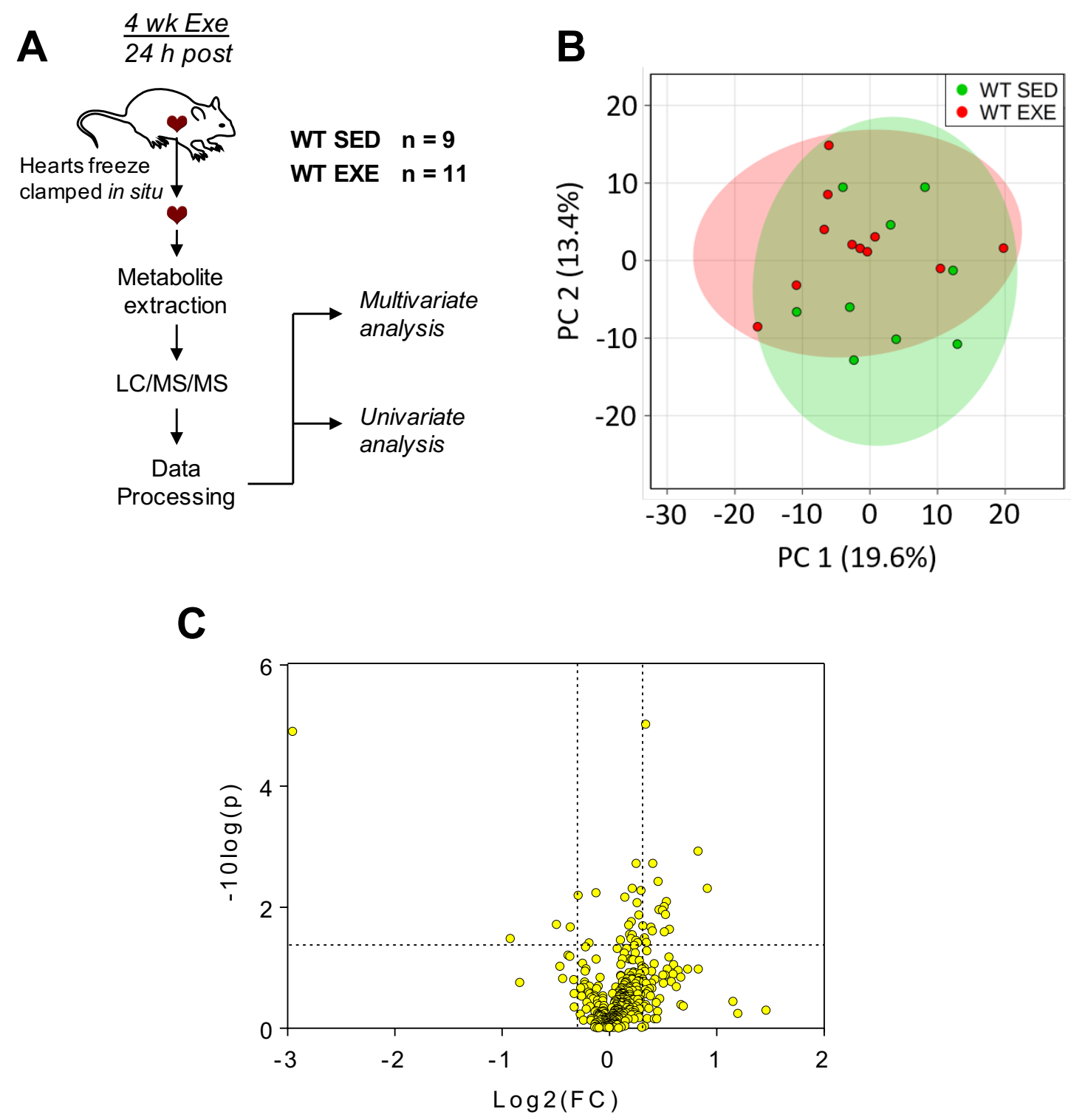
Table 4: Significantly different metabolites in hearts from exercise-adapted mice. Hearts from WT, exercise-adapted (EXE) mice [(and corresponding WT sedentary controls (SED)] were freeze-clamped in situ, $24 \mathrm{~h}$ after the final exercise bout. Metabolites extracted from the hearts were subjected to LC/MS analysis. Raw area counts from each identified metabolite were log-transformed, autoscaled, and then subjected to t-test analysis. Missing values were imputed using half the minimum value of observed raw area count data, and metabolites with $>50 \%$ values missing were omitted from the analysis. Asterisks $\left(^{*}\right)$ indicate compounds that were not officially confirmed based on a standard, but whose identity matches the expected exact mass using the UHPLC/MS/MS ${ }^{2}$ accurate mass platform. Shown are those metabolites with a $p$ value threshold of 0.05 and a fold change threshold of 1.25. $n=9$ SED mice and $n=10$ EXE mice. 
TABLE 4

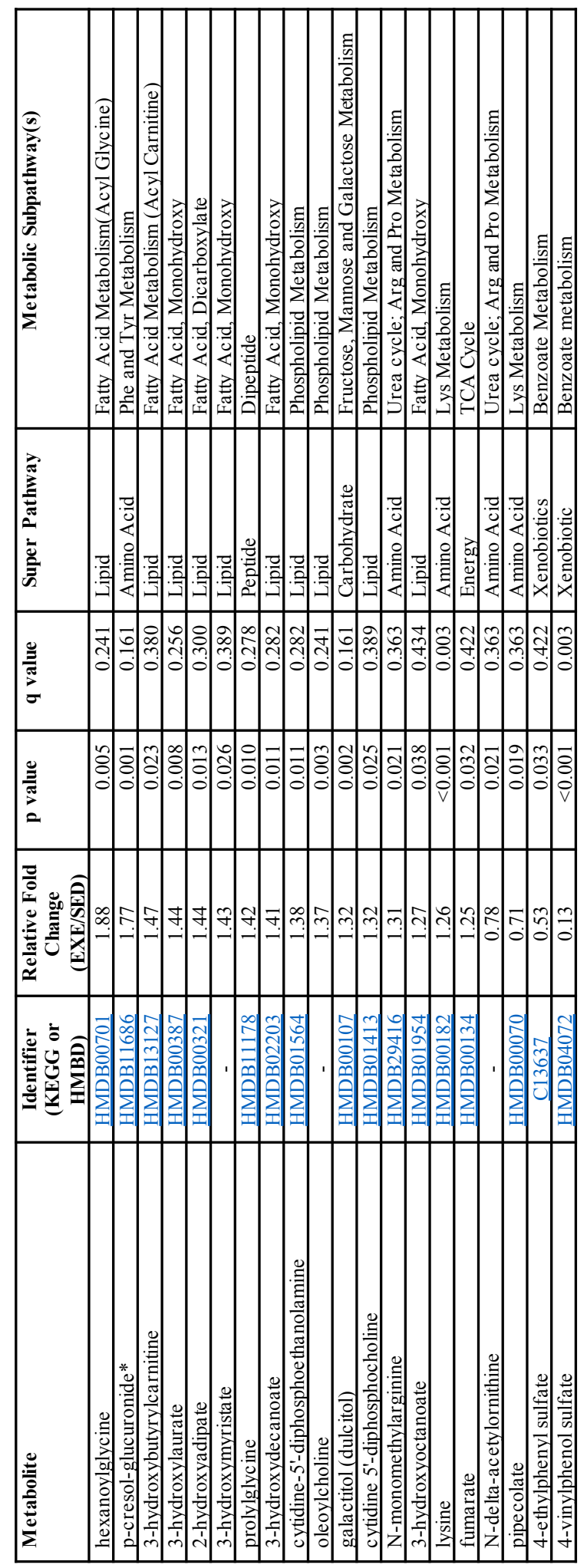


the steady-state abundance of most metabolites in the exercise-adapted heart is unchanged compared with that of sedentary mice.

Exercise induces periodicity in glucose metabolism. Because the steady-state abundance of biochemical pathway intermediates may not be reflective of pathway flux, ${ }^{222,232}$ and because several studies show that exercise changes cardiac metabolism (e.g. ${ }^{40,66}$ ), we next measured the metabolism of $\left[5-{ }^{3} \mathrm{H}\right]$ glucose in hearts from sedentary and exercise-adapted mice. Radiometric assays of perfused hearts showed a nearly 2fold increase in glucose utilization in exercise-adapted hearts compared with hearts from sedentary mice (Fig. 13A). Glycolytic enzymes were found in similar abundances in exercise-adapted hearts relative to hearts from sedentary mice (Fig. 14). These data indicate that changes in enzyme abundance are unlikely to contribute to the increase in glycolysis that occurs in the exercise-adapted heart.

Because glycolysis is influenced by rate-limiting steps, such as occurs at the phosphofructokinase reaction, we next examined factors that regulate PFK1the rate-limiting and committed step of glycolysis. ${ }^{233,234}$ The PFK1 reaction exerts more control on glycolysis than any other $\operatorname{step}^{235}$ and is dependent on several factors including: the levels of fructose-2,6,-bisphosphate $\left(F-2,6-P_{2}\right)$, substrate availability, energy charge, the levels of citrate and ATP, and pH. Because PFK2 regulates the levels of $\mathrm{F}-2,6-\mathrm{P}_{2}$, a potent activator of PFK1, we examined the phosphorylation status of the myocardial isoform of PFK2, PFKFB2. Phosphorylation of PFKFB2 at S483 can increase F-2,6- $\mathrm{P}_{2}$ levels and is known to 
be under the influence of insulin-like growth factor-1/AKT signaling. ${ }^{35,236-238}$ Notably, both cardiac AKT phosphorylation and plasma insulin-like growth factor1 were elevated in exercise-adapted mice (see Fig. 11E and Fig. 10D). In agreement with radiometric findings, S483 phosphorylation of PFKFB2 ( $p-P F K 2$ ) was $50 \%$ higher in exercise-adapted hearts compared with hearts of sedentary mice (Fig. 13B, upper panel and 13C, left panel). Taken together, the radiometric and immunologic data provide convergent evidence that glycolysis is increased in the exercise-adapted heart and indicate that this increase in glycolytic activity may be attributable at least in part to PFK activation.

Because exercise is a dynamic challenge that leads to rapid changes in metabolism, we next examined glycolytic changes that occur early after exercise, i.e., in the trained state during recovery. Because ex vivo measurements of flux in the recovery period following an exercise session are unlikely to provide meaningful data (e.g., due to lack of a metabolic steady-state), we measured levels of p-PFK2 as a possible indicator of phosphofructokinase activity and glucose utilization through glycolysis. Compared with corresponding sedentary controls, hearts excised from exercise-adapted mice $1 \mathrm{~h}$ after the last exercise session showed significantly diminished PFK2 phosphorylation (Fig. 13B, lower panel and $13 \mathrm{C}$, right panel).

To determine whether these changes in metabolism precede cardiac adaptation, we eliminated the variable of extended training and examined PFK2 activation immediately after a single bout of exercise. For this, a separate group of 
Figure 13: Exercise dynamically regulates PFK2 and glycolysis in the heart. Measurements of glucose metabolism in the heart: (A) Measurement of relative myocardial glucose utilization in isolated perfused hearts from mice that remained sedentary (Sed) or that were exercised for 4 weeks (Exe). Measurements were performed on hearts excised $24 \mathrm{~h}$ after the last exercise session; (B) Representative immunoblots from a separate cohort of mice of S483 phosphorylation of the cardiac isoform of PFK2 (PFKFB2) in the sedentary and the exercise-adapted state: upper panel, levels of PFK2 phosphorylation $24 \mathrm{~h}$ after the last bout of exercise; lower panel, PFK2 phosphorylation occurring $1 \mathrm{~h}$ after the last exercise bout. (C) Quantification of PFK2 phosphorylation from blots represented in panel B; (D) Representative immunoblots of S483 phosphorylation of PFK2 in hearts of mice exercised for $1 \mathrm{~d}$; these hearts were excised immediately after exercise, which would appear to be indicative of the state of PFK2 occurring during or immediately after exercise; (E) Schematic representation of glycolysis showing relationship of PFK activity with glycogen storage; (F) Myocardial glycogen content in hearts of Sed mice and in hearts excised immediately after 1 bout of exercise; Circulating levels of $(\mathbf{G})$ blood glucose, $(\mathbf{H})$ blood lactate, and (I) plasma free fatty acids (FFA) in mice subjected to a single bout of exercise; (J) Schematic illustrating changes in glucose utilization occurring with acute and chronic exercise, i.e., exercise-induced metabolic periodicity. Data are represented as mean \pm SEM; ${ }^{*} p<0.05,{ }^{* *} p<0.01,{ }^{* * *} p<0.0001 ; n=5-8$ per group. 
FIGURE 13

\section{Chronic Exercise: 4 wk "Adapted"}

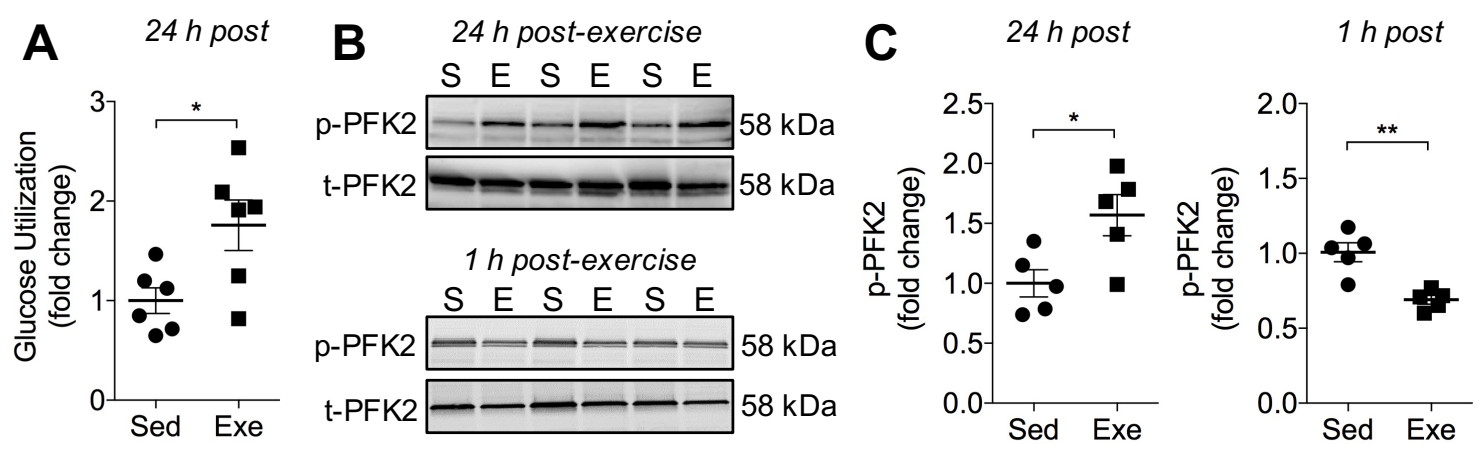

Acute Exercise: $1 d$ "During Exe"

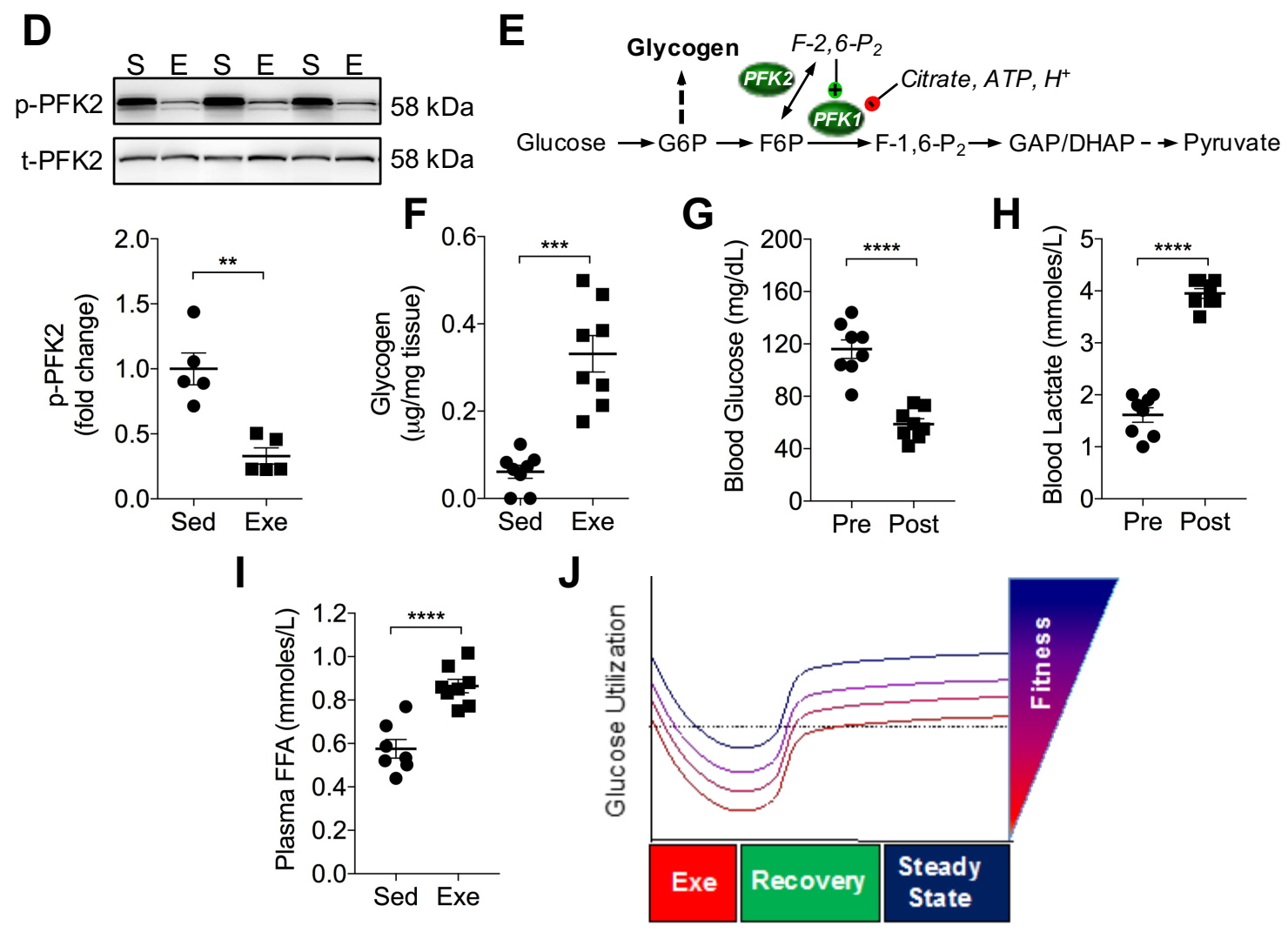


Figure 14: Exercise training does not alter the abundance of key glucosemetabolizing enzymes. Western blot analysis of enzymes in glycolysis and glucose metabolism: (A) Representative western blots of hexokinase 2 (HK2), phosphofructokinase (PFK1), phosphofructo-kinase/fructose-bisphosphatase 2 (PFKFB2; PFK2), phosphofructo-kinase/fructose-bisphosphatase 3 (PFKFB3; PFK2), pyruvate kinase (PKM1/2), pyruvate dehydrogenase kinase 1 (PDHK1), glutamine:fructose-6-phosphate aminotransferase 1 (GFAT1), glycogen synthase (GS), and lactate dehydrogenase b (LDHB); (B) Quantification of western blots from A. Data are represented as mean \pm SEM; $n=6$ per group; in all cases, $p>0.05$ after Bonferroni correction. 


\section{FIGURE 14}

A

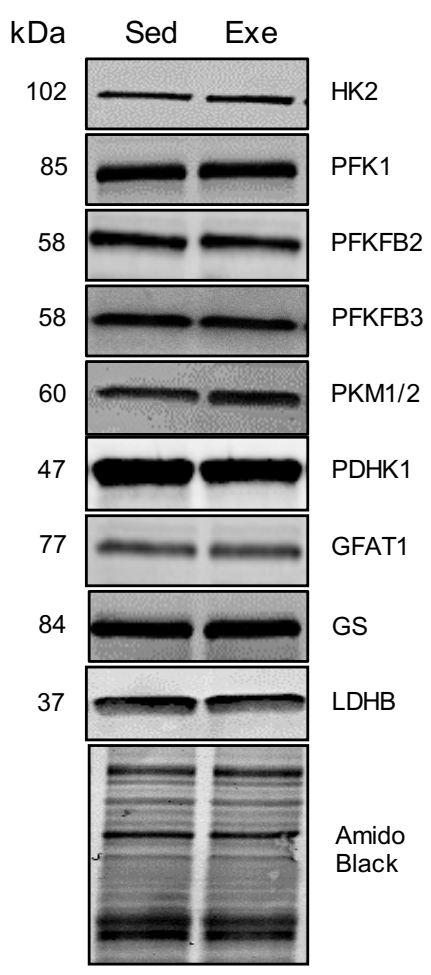

B
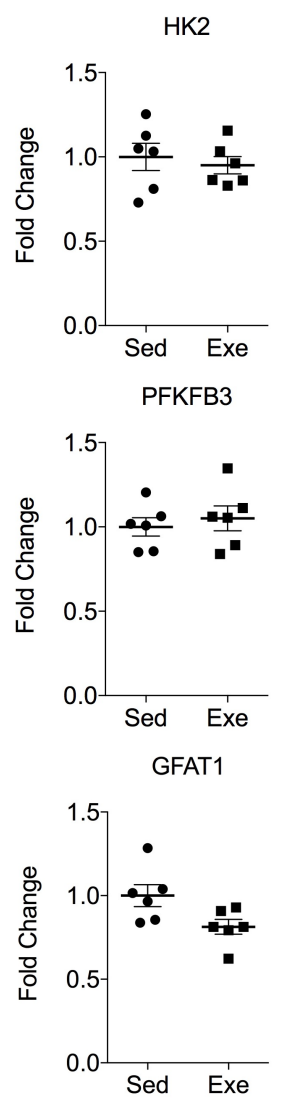

PFK1
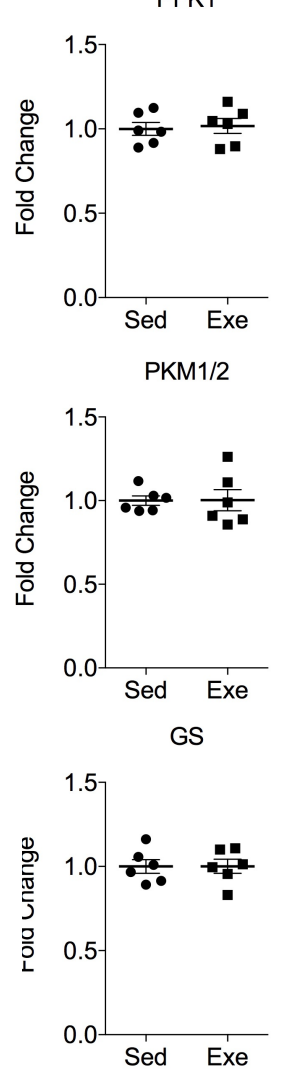

PFKFB2
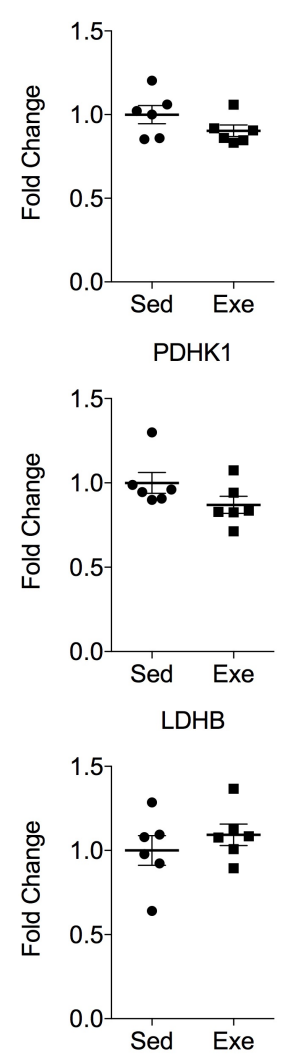
mice was subjected to a single bout of exercise for $40 \mathrm{~min}$. Immediately following this exercise bout, mice were euthanized and the hearts were excised for biochemical analysis. We found that p-PFK2 levels in these hearts were remarkably lower than those in sedentary controls (Fig. 13D), suggesting that a bout of exercise might acutely decrease the rate of glycolysis in the heart. Because measurement of glycolytic rate ex vivo would likely obfuscate the metabolic changes that occur during or shortly after a bout of exercise (due to loss of hemodynamic, metabolic, and paracrine [e.g. catecholamine] influence), we chose to measure glycogen content as an additional surrogate of PFK activity and glycolysis. Thus, we hypothesized that, during exercise, PFK-mediated decreases in glycolytic rate should promote glycogen accumulation (Fig. 13E), which is consistent with the notion that decreases in PFK activity promote glycogenesis. ${ }^{239}$ Indeed, glycogen content was elevated $>5$-fold in hearts collected immediately after a single bout of exercise (Fig. 13F). Notably, this elevation in cardiac glycogen is not related to the short $(6 \mathrm{~h})$ fasting period occurring in sedentary and exercised mice because independent experiments showed that fasting mice for this duration does not affect myocardial glycogen content (unfasted, $0.17 \pm 0.03$ $\mu \mathrm{g} / \mathrm{mg}$ tissue; $6 \mathrm{~h}$ fasted, $0.18 \pm 0.05 \mu \mathrm{g} / \mathrm{mg}$ tissue; $\mathrm{n}=7-8$ per group).

Because substrate availability and delivery are key determinants of myocardial substrate preference and utilization, ${ }^{75,78,79,100}$ we measured the circulating levels of glucose, lactate, and free fatty acids in mice immediately following a bout of exercise. Compared with pre-exercise levels, mice subjected to 40 min of treadmill exercise showed a $50 \%$ decrease in blood glucose and a 2.5 - 
fold increase in blood lactate (Fig. 13G,H). Moreover, plasma free fatty acids were significantly elevated (Fig. 13I), which along with the changes in blood glucose and lactate, would stimulate lactate and fat oxidation and diminish overall glucose utilization. These data are concordant with our biochemical measurements and provide convergent evidence suggesting that, during and immediately after exercise, the rate of myocardial glycolysis is diminished; however, upon adaptation and full recovery, the steady-state rates of glycolysis appear to be increased in the heart (Fig. 13J).

Phosphofructokinase is a strong regulator of the glucose-fatty acid cycle in the heart. We next examined whether exercise-induced periodicity in glucose metabolism might be essential for structural and functional cardiac adaptation. This hypothesis is consistent with the notion that repetitive bouts of exercise are a trigger for the transcriptional adaptation in skeletal muscle. ${ }^{8}$ As tools to examine the relationship between changes in myocardial glucose metabolism and cardiac remodeling, we used genetically modified mice expressing kinase-deficient $\left(\mathrm{Glyco}^{\mathrm{LO}}\right)$ or phosphatase-deficient $\left(\mathrm{Glyco}^{\mathrm{Hi}}\right)$ point-mutant isoforms of PFK2 under the control of the $\alpha-\mathrm{MHC}$ promoter, which constitutively decrease or increase PFK1 activity and myocardial glycolytic rate. ${ }^{220,} 221$ Compared with cardiomyocytes isolated from WT mice, the abundance of F-2,6- $\mathrm{P}_{2}$ was $>2$-fold lower in Glyco Lo cardiomyocytes and $>5$-fold higher in Glyco ${ }^{\text {Hi }}$ cardiomyocytes (Fig. 15A). The rate of glycolysis in perfused hearts was in accordance with transgene-induced alterations in F-2-6-P2 (Fig. 15B). Consistent with the notion that PFK activity 
regulates glycogen storage, ${ }^{239} \mathrm{Glyco}^{\mathrm{LO}}$ hearts showed 1.6-fold higher myocardial glycogen content, and $\mathrm{Glyco}^{\mathrm{Hi}}$ hearts demonstrated 4-fold lower levels of glycogen (Fig. 15C).

To determine how glycolysis affects myocardial metabolism, we measured the steady-state abundance of myocardial metabolites in both $\mathrm{Glyco}^{\mathrm{Lo}}$ and Glyco $\mathrm{Hi}^{\mathrm{Hi}}$ hearts (Fig. 15D). PCA showed that the relative abundances of metabolites could discriminate between the genotypes (Fig. 15E). Volcano plot analysis demonstrated that, out of the 424 metabolites measured, 108 metabolites in Glyco $^{\text {Lo }}$ hearts (Fig. 16A, Table 5) and 109 metabolites in Glyco ${ }^{\text {Hi }}$ hearts (Fig. 16B, Table 6) were significantly different from WT hearts. Visualization of changes in the metabolic network demonstrated the greatest impact on the lipid superfamily, with acylcarnitines (subfamily 1 ) showing robust, diametrically opposite changes in the Glyco ${ }^{\text {Lo }}$ and $\mathrm{Glyco}^{\mathrm{Hi}}$ hearts (Fig. 15F, G). Z-score analyses illustrate remarkable increases in medium and long chain acylcarnitines in $\mathrm{Glyco}^{\mathrm{Hi}}$ hearts; the corresponding species were lower in abundance in Glyco ${ }^{\text {Lo }}$ hearts (Fig. 17A and Tables 5, 6). In addition, long chain fatty acids (saturated and unsaturated) were lower in Glyco ${ }^{\mathrm{LO}}$ mice, while many of the same species were elevated in Glyco $^{\text {Hi }}$ mice (Fig. 15F, G, Fig. 17A, and Tables 5, 6). Interestingly, Glyco ${ }^{L o}$ hearts demonstrated elevated levels of aconitate, homocitrate, and a-ketoglutarate (Fig. 15F, G and Table 5), suggesting that high rates of lipid oxidation in these hearts might flood the Krebs cycle. Glycolytic intermediates were lower in $\mathrm{Glyco}^{\mathrm{Hi}}$ mice, while most glycolytic intermediates were unchanged in abundance in Glyco ${ }^{\mathrm{LO}}$ 


\section{Figure 15: Cardiac $\mathrm{F}-2,6-\mathrm{P}_{2}$ regulates myocardial glucose and lipid}

metabolism. Metabolic phenotyping of $\mathrm{Glyco}^{\mathrm{Lo}}$ and Glyco ${ }^{\mathrm{Hi}}$ mice: (A) Levels of F2,6- $\mathrm{P}_{2}$ in cardiomyocytes isolated from $\mathrm{WT}$, Glyco ${ }^{\mathrm{Lo}}$, and Glyco ${ }^{\mathrm{Hi}}$ mice. $\mathrm{N}=3-6$ per group; (B) Relative myocardial glucose utilization in isolated perfused hearts. $n=$ 5-7 per group; (C) Myocardial glycogen content. N = 7-9 per group; (D) Schematic of 106hosphor106ics analysis of WT, Glyco ${ }^{\text {Lo }}$, and Glyco ${ }^{\text {Hi }}$ hearts; (E) 2D PCA analysis; (F) Metabolic network visualization of metabolites in $\mathrm{Glyco}^{\mathrm{LO}}$ and $\mathrm{Glyco}^{\mathrm{Hi}}$ hearts, compared with corresponding WT hearts. Circle size is indicative of the magnitude of change (blue and red circles indicate significantly decreased or increased metabolites abundance compared with WT controls, respectively). Numbers represent metabolites in the following subpathways: 1 : Acylcarnitines; 2 : Monacylglycerols; 3: Monohydroxy fatty acids; 4: Phospholipid metabolism; 5: Lysolipids; 6: Polyunsaturated fatty acids; 7: Sphingolipids; 8: Dicarboxylic fatty acids; 9: Long chain fatty acids; 10: Glycolysis; 11: Fructose, Mannose, Galactose metabolism; 12: Pentose metabolism; 13: TCA cycle; 14: Purine metabolism, (hypo)xanthine/inosine; 15: Purine metabolism, adenine; 16: Gly, Ser, Thr metabolism; 17: BCAA metabolism; 18: Met, Cys, SAM, Tau metabolism; (G) Overview of changes in major metabolic subpathways in Glyco ${ }^{\mathrm{LO}}$ and $\mathrm{Glyco}^{\mathrm{Hi}}$ hearts $(F) .{ }^{*} p<0.05,{ }^{* *} p<0.01,{ }^{* * *} p<0.001,{ }^{* * *} p<0.0001$ vs. the indicated group. 
A

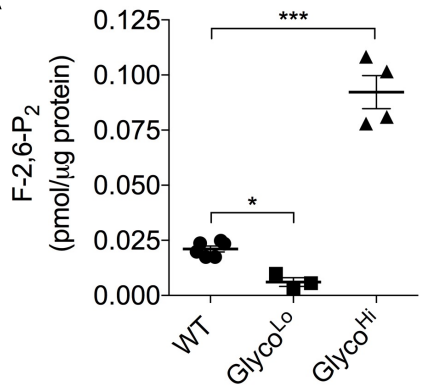

D

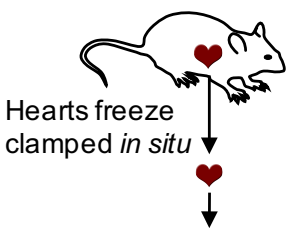

Metabolite extraction

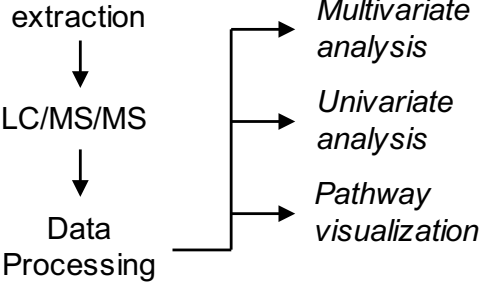

$\mathbf{F}$

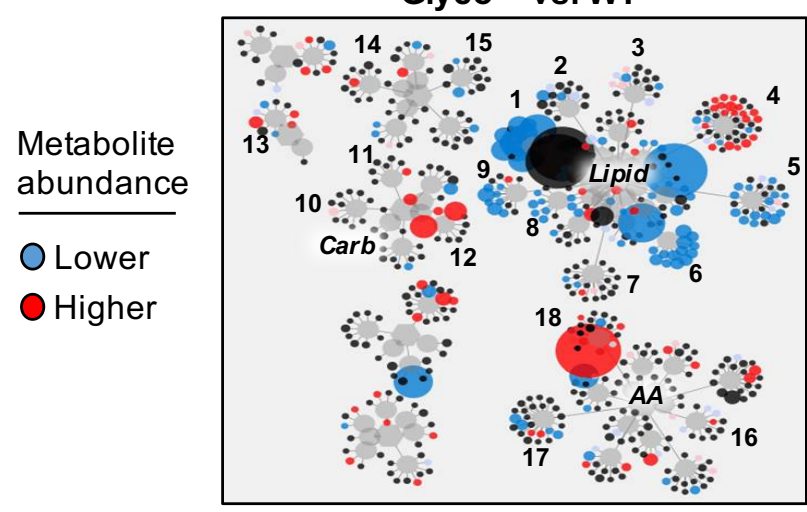

G

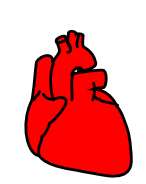

Glyco ${ }^{\text {LO }}$

E

GlycoLo $n=10$

GlycoHi $^{\mathrm{H}}=\mathbf{1 0}$

$\downarrow \downarrow \downarrow$ acylcarnitines (1)

$\downarrow \downarrow$ fatty acids $(3,6,8,9)$

$\uparrow$ glycogen intermediates (11)

$\uparrow$ Krebs cycle intermediates (13)

$\uparrow$ Phospholipids (4)

$\leftrightarrow$ glycolytic intermediates (10)
B

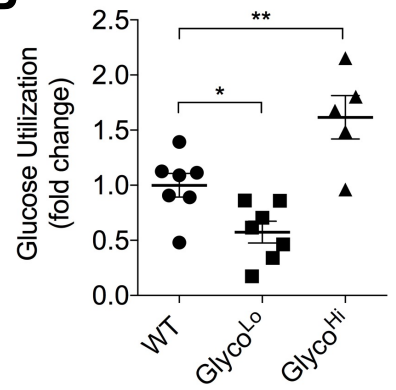

C

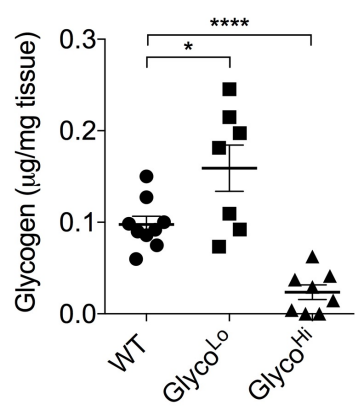

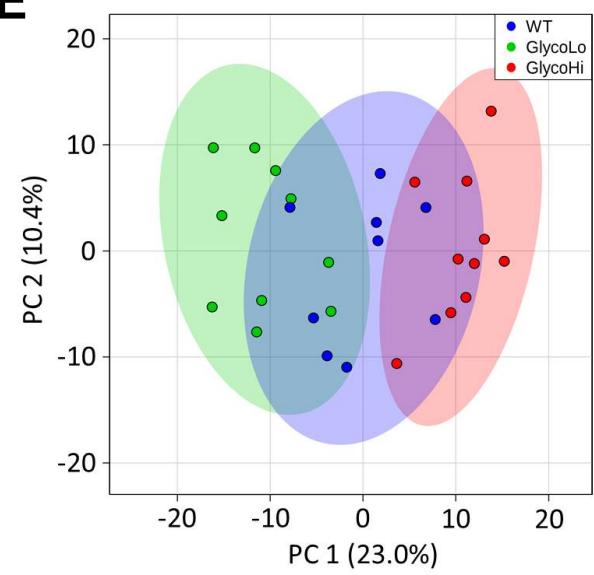
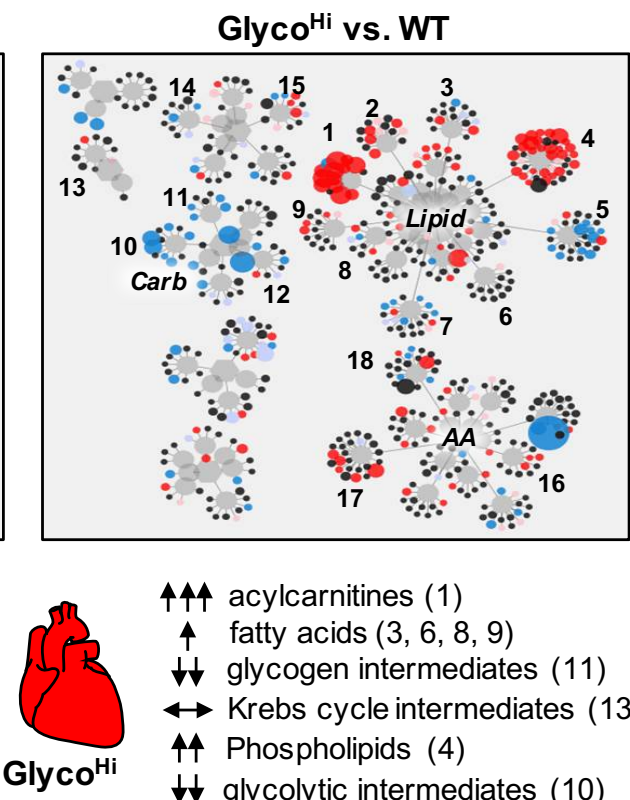

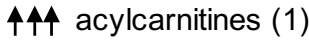

$\uparrow$ fatty acids $(3,6,8,9)$

$\downarrow$ glycogen intermediates (11)

$\leftrightarrow$ Krebs cycle intermediates (13)

个4 Phospholipids (4)

$\downarrow \downarrow$ glycolytic intermediates (10) 
Figure 16: Metabolomic changes in the $\mathrm{Glyco}^{\mathrm{LO}}$ and $\mathrm{Glyco}^{\mathrm{Hi}}$ mouse hearts. Following a $6 \mathrm{~h}$ fast, hearts were freeze-clamped in situ. The relative abundance of metabolites in the hearts was measured by LC/MS/MS. Volcano plot showing metabolites that changed $\geq 1.25$-fold $(p \leq 0.05)$ in $(A)$ Glyco $^{\text {Lo }}$ and $(B) G^{\prime}$ Glyco $^{\mathrm{Hi}}$ hearts compared with WT hearts; A list of metabolites found to be significantly different in these hearts can be found in Tables S3 and S4. N = 9 WT, 10 Glyco $^{\text {Lo }}$, $10 \mathrm{Glyco}^{\mathrm{Hi}}$ mouse hearts per group. 
A

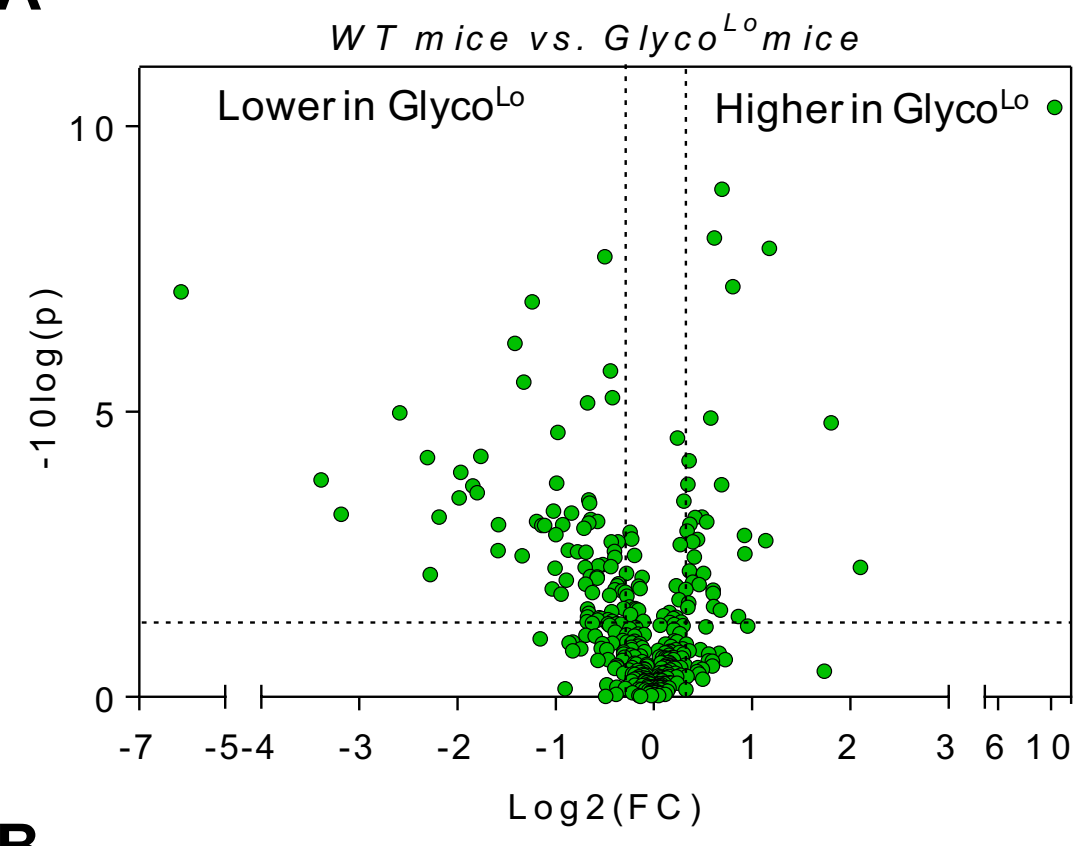

B

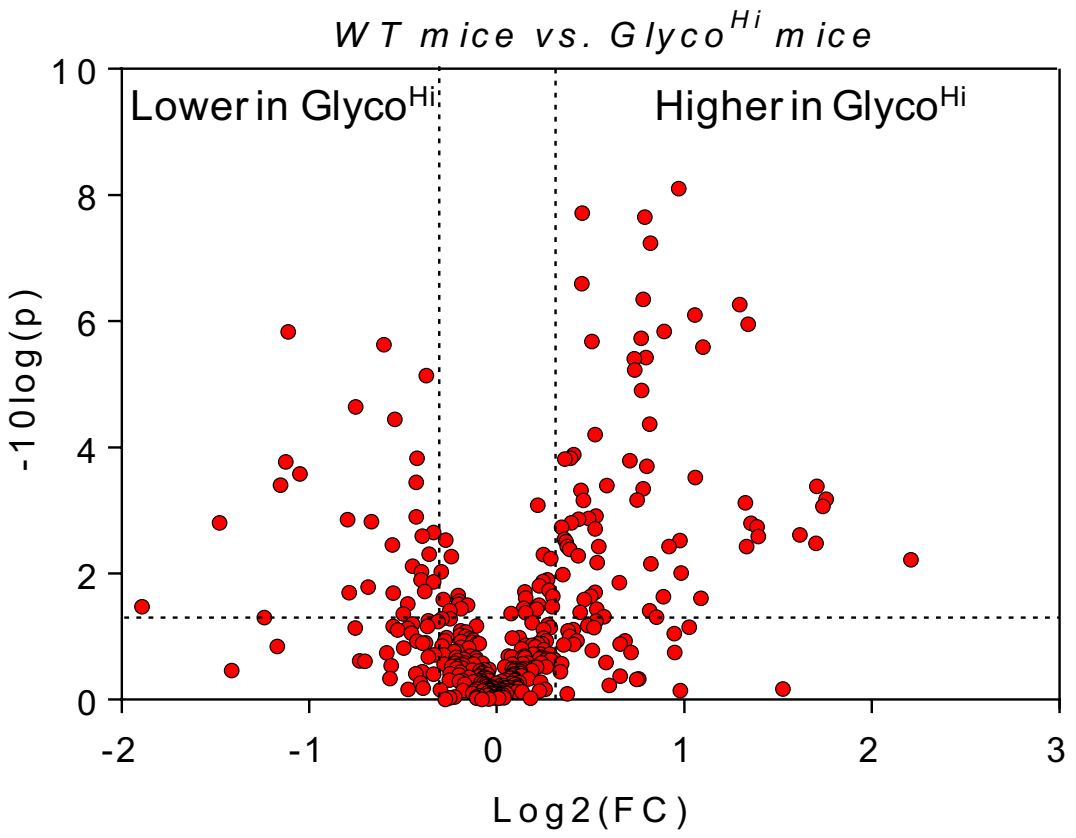


Table 5: Significantly different metabolites in hearts from WT and Glyco ${ }^{\text {Lo }}$ mice. Metabolites extracted from the hearts of WT and cardiac-specific kd-PFK2 transgenic mice (Glyco ${ }^{\text {Lo }}$ mice) were subjected to LC/MS analysis. Raw area counts from each identified metabolite were log-transformed, autoscaled, and then subjected to statistical analyses (t-test). Missing values were imputed using half the minimum value of observed raw area count data, and metabolites with $>50 \%$ values missing were omitted from the analysis. Asterisks $\left(^{*}\right)$ indicate compounds that were not officially confirmed based on a standard, but whose identity matches the expected exact mass using the UHPLC/MS/MS ${ }^{2}$ accurate mass platform. Shown are those metabolites with a $p$ value threshold of 0.05 and a fold change threshold of 1.25. $n=9$ WT mice and $n=10 \mathrm{Glyco}^{\mathrm{Lo}}$ mice. ${ }^{\text {\#}}$ S-methylmethionine was not detected in any of the WT samples, and campesterol was detected only in one WT sample; thus, the relative fold change values are based upon imputed values (i.e., half the minimum value reported for the particular metabolite). 
TABLE 5

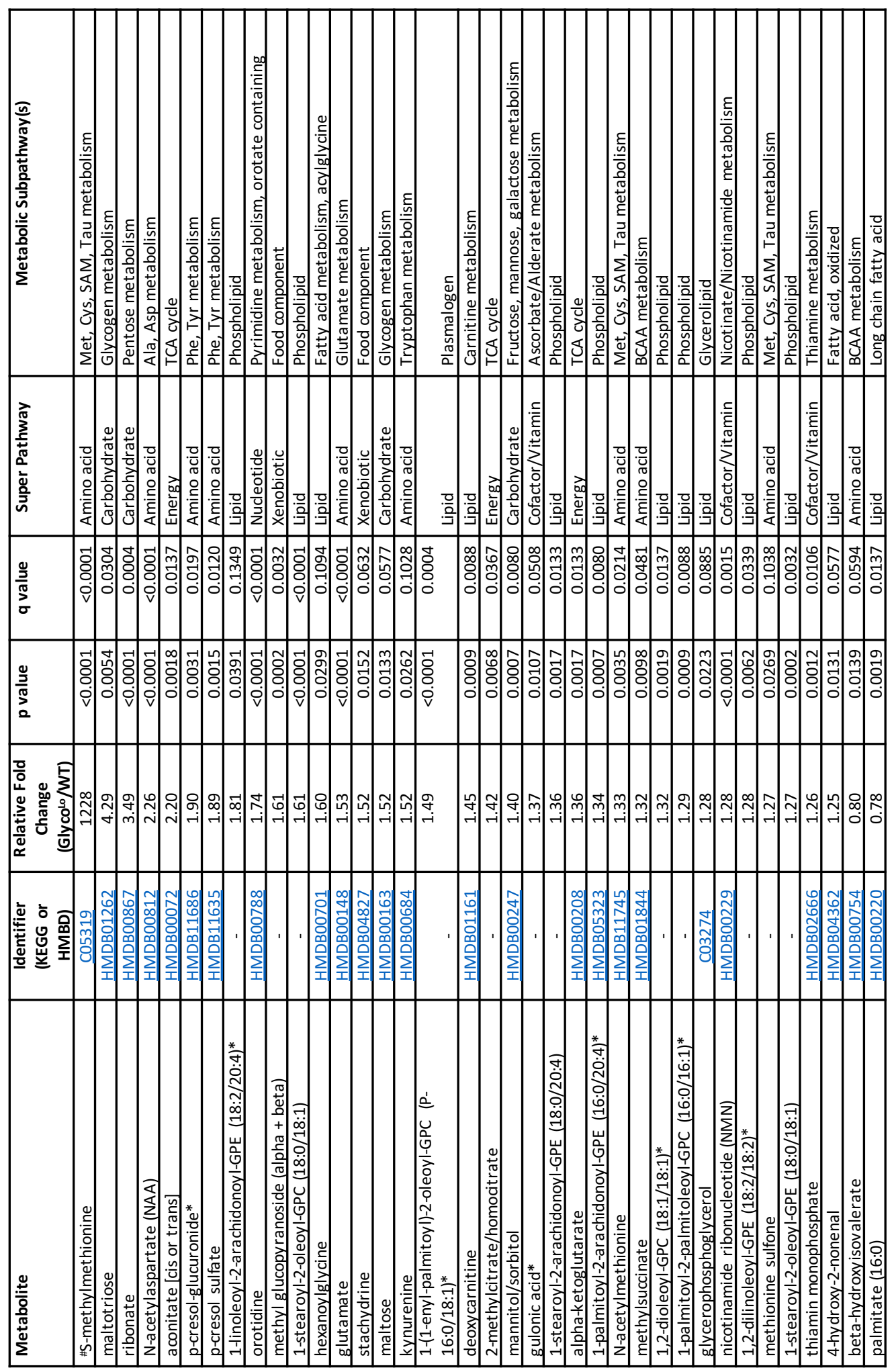


TABLE 5

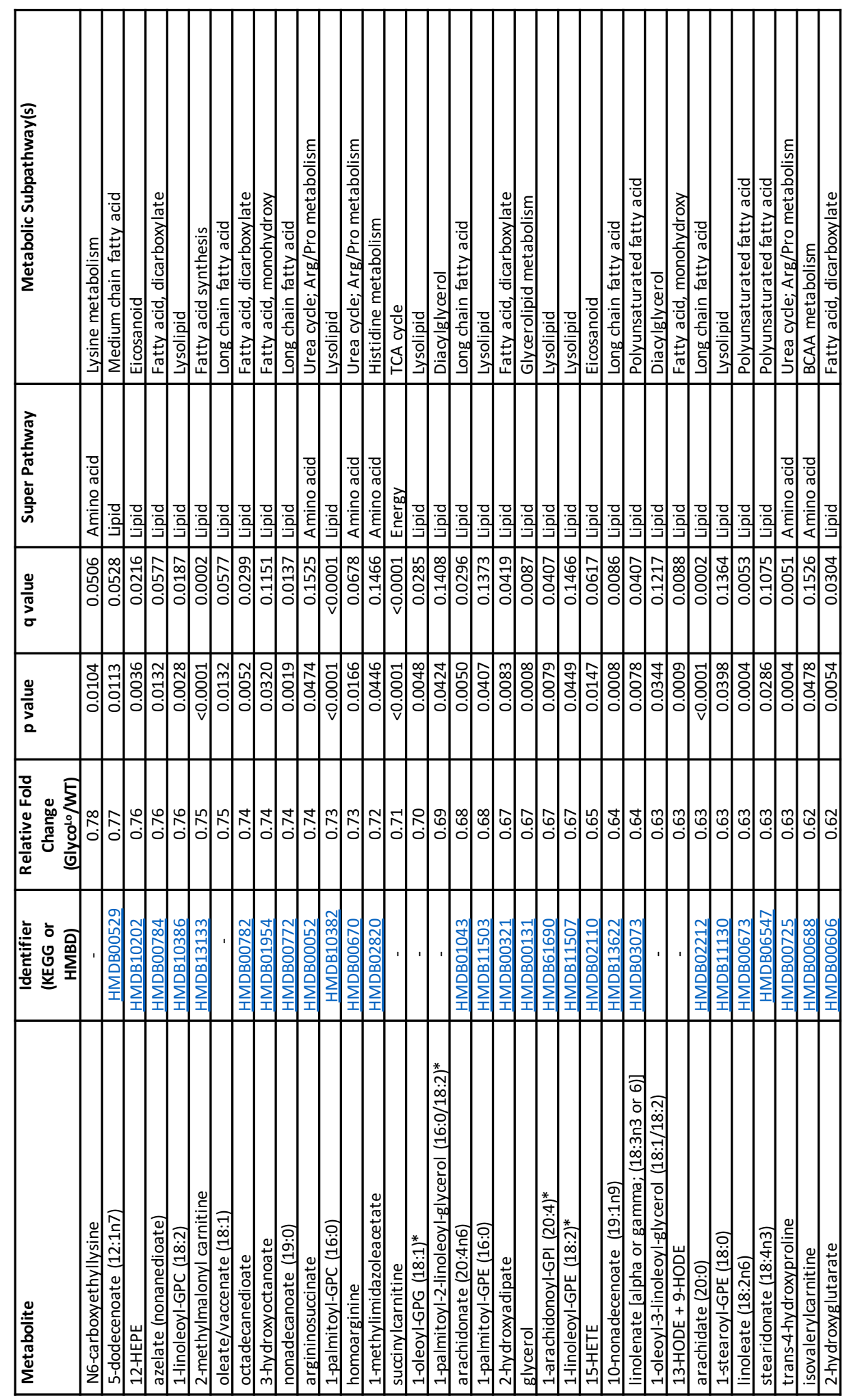


TABLE 5

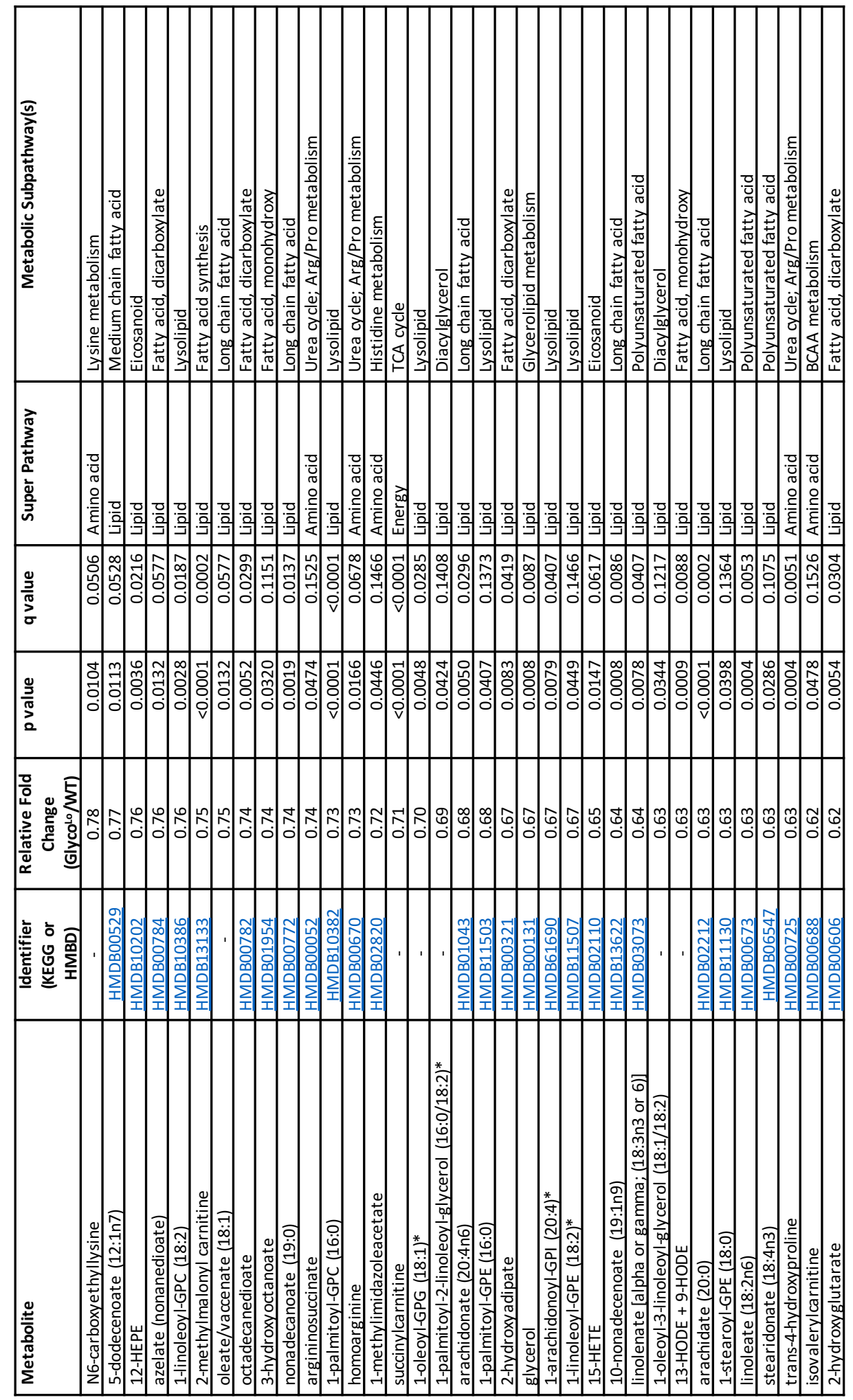


Table 6: Significantly different metabolites in hearts from WT and Glyco ${ }^{\mathrm{Hi}}$ mice. Metabolites extracted from the hearts of WT and cardiac-specific pd-PFK2 transgenic mice (Glyco ${ }^{\mathrm{Hi}}$ mice) were subjected to LC/MS analysis. Raw area counts from each identified metabolite were log-transformed, autoscaled, and then subjected to t-test analysis. Missing values were imputed using half the minimum value of observed raw area count data, and metabolites with $>50 \%$ values missing were omitted from the analysis. The asterisk $\left(^{*}\right)$ indicates compounds that were not officially confirmed based on a standard, but whose identity matches the expected exact mass using the UHPLC/MS/MS ${ }^{2}$ accurate mass platform. Shown are those metabolites with a $p$ value threshold of 0.05 and a fold change threshold of 1.25. $n=9$ WT mice and $n=10 \mathrm{Glyco}^{\mathrm{Hi}}$ mice. 
TABLE 6

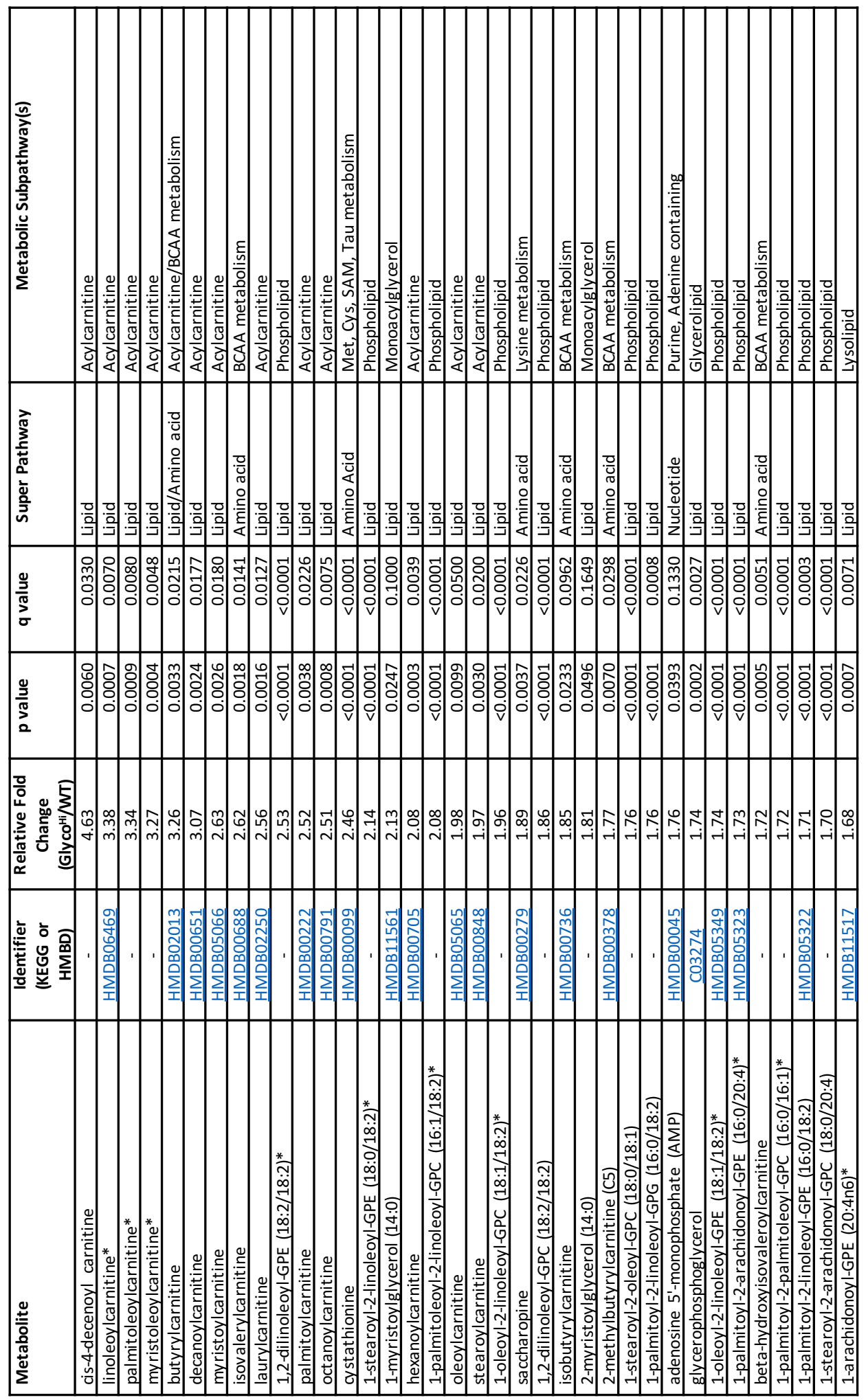


TABLE 6

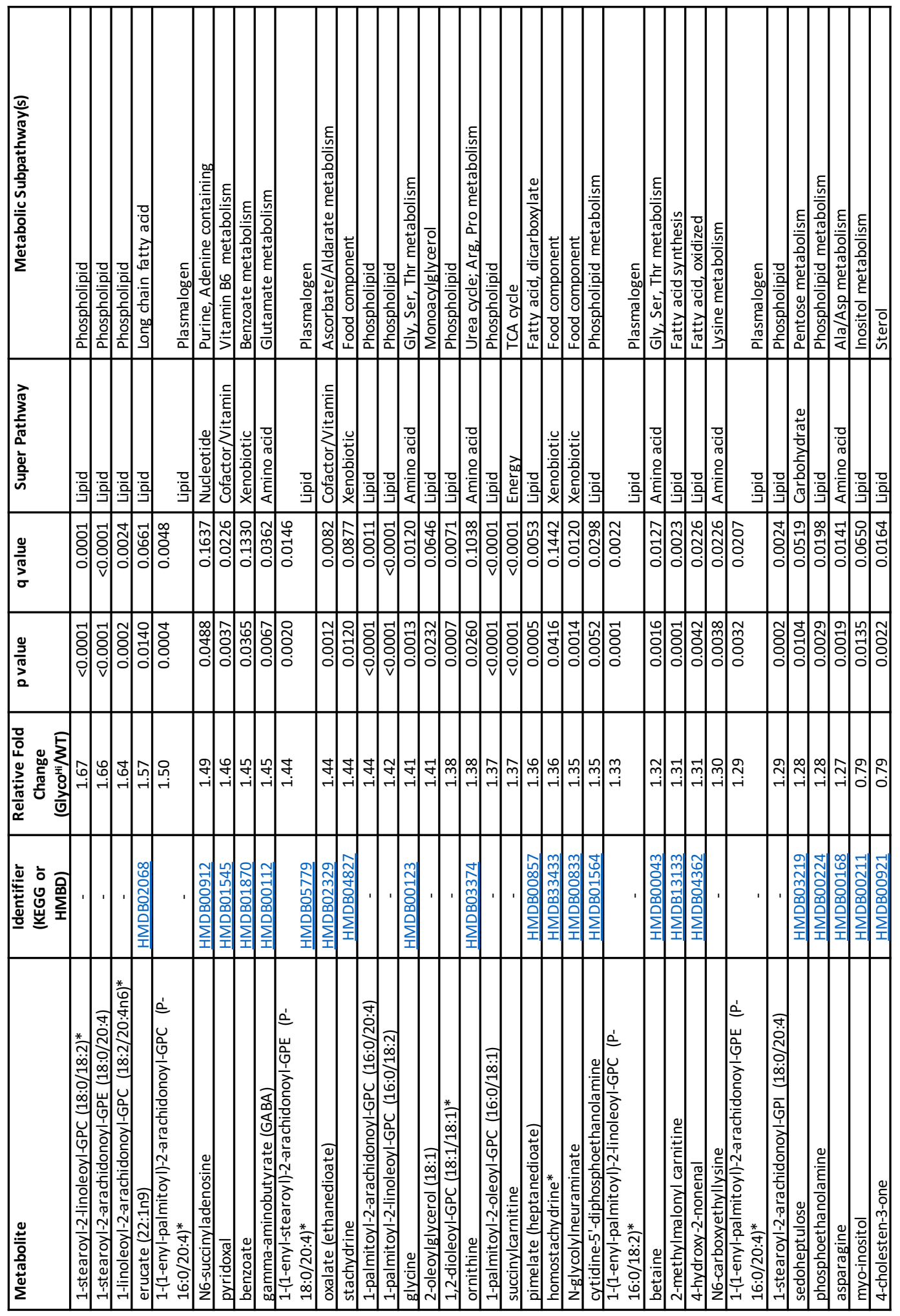


TABLE 6

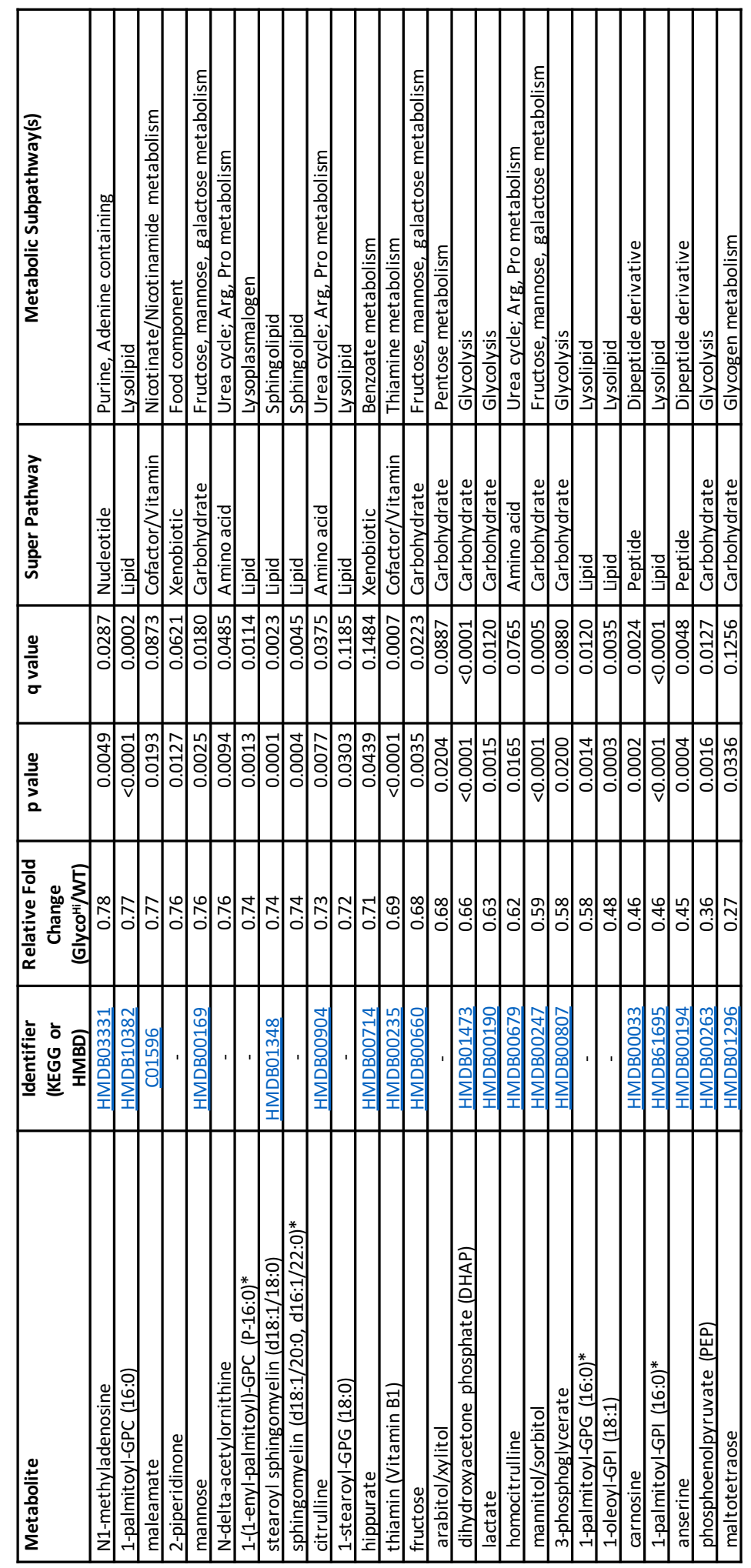


Fig 17: Modulation of cardiac glycolytic rate regulates lipid, carbohydrate, and amino acid metabolism. Spider charts showing average z-scores of (A) lipid (B) carbohydrate and (C) amino acid and peptide metabolites that were significantly different in Glyco ${ }^{\mathrm{LO}}$ or Glyco ${ }^{\mathrm{Hi}}$ hearts compared with WT controls. $\mathrm{N}=$ 9 WT, 10 Glyco $^{\text {Lo }}, 10$ Glyco $^{\text {Hi }}$ mouse hearts per group. 
FIGURE 17

A

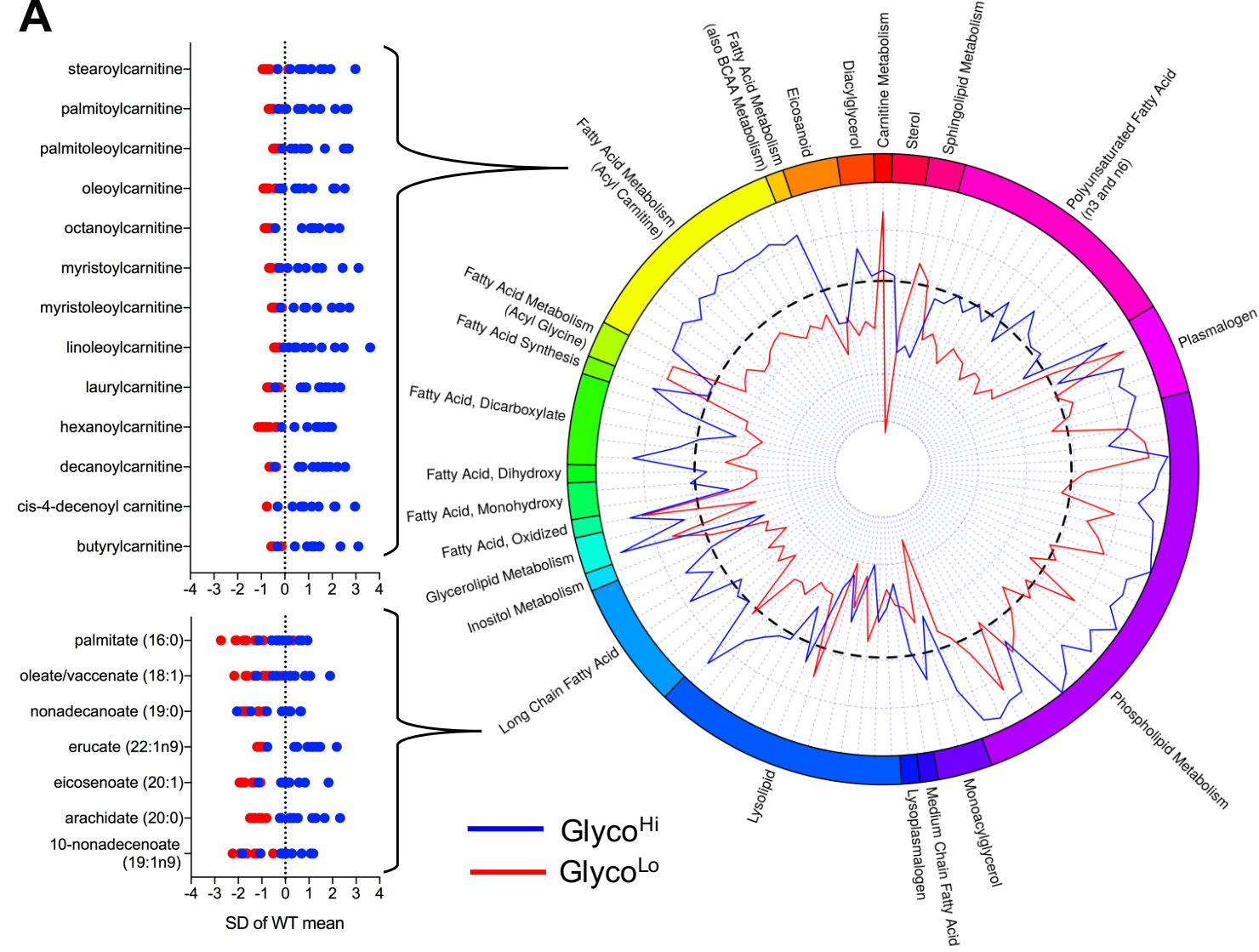


FIGURE 17

B

C
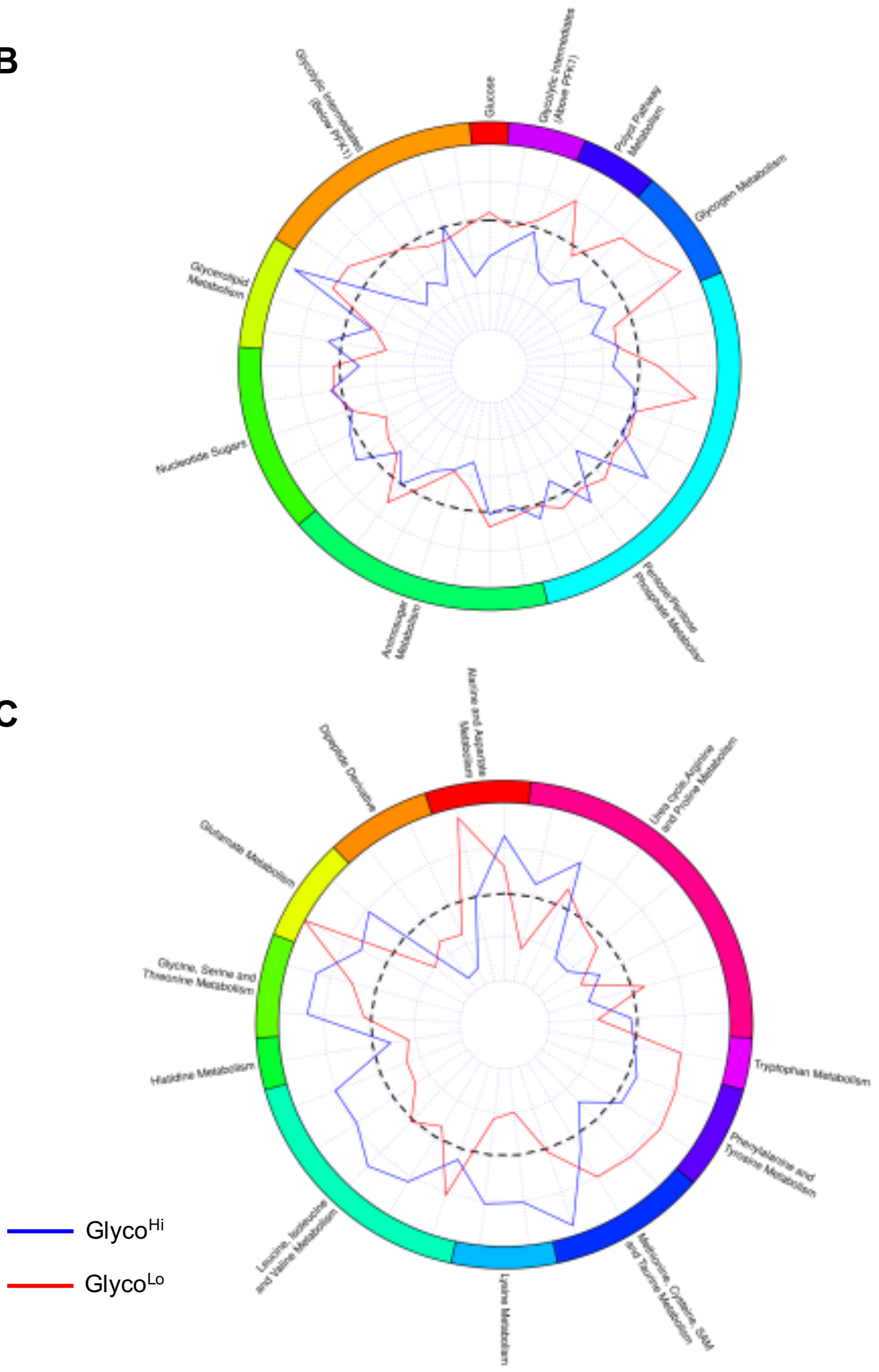
mice (Fig. 15F, G and Fig. 17B). Changes in the steady-state abundances of glycolytic and lipid metabolites are most consistent with increased propensity for glucose oxidation in Glyco ${ }^{\mathrm{Hi}}$ hearts and increased lipid oxidation in Glyco ${ }^{\mathrm{LO}}$ hearts. These changes would be sufficient to explain the accumulation of acylcarnitines and diminished levels of glycolytic intermediates occurring in Glyco $^{\text {Hi }}$ hearts, and the lower levels of acylcarnitines in Glyco ${ }^{\text {Lo }}$ hearts.

Polarization of myocardial metabolism also resulted in modest changes in amino acid metabolites (see Fig. 17C and Tables 5, 6). Collectively, these results show the high degree to which phosphofructokinase regulates the glucose-fatty acid cycle in the heart.

Constitutively low glycolytic activity promotes structural and transcriptional changes consistent with the exercise-adapted heart. To examine whether PFK activity regulates cardiac growth, we assessed the structure and function of Glyco $^{\mathrm{LO}}$ and $\mathrm{Glyco}^{\mathrm{Hi}}$ hearts. Both the $\mathrm{Glyco}^{\mathrm{LO}}$ and $\mathrm{Glyco}^{\mathrm{Hi}}$ hearts showed a mild increase in cardiac size, which was associated with increased cardiomyocyte cross-sectional area (Fig. 18A-C, E, F); however, significantly increased capillaryto-myocyte ratio was observed only in Glyco ${ }^{\text {Lo }}$ hearts (Fig. 18A, D). Picrosirius red staining showed no noteworthy myocardial fibrosis in either strain (Fig. 18A). Interestingly, Glyco ${ }^{\text {Lo }}$ hearts showed elevated EDV and slightly increased wall thicknesses compared with WT mice (Table 7), which is similar to that found in WT, exercise-adapted mice (see Table 3). In contrast, Glyco ${ }^{\mathrm{Hi}}$ hearts had 
Figure 18: Constitutive changes in glycolysis promote cardiac growth and hypertrophy. Structural and molecular indices of cardiac growth/hypertrophy: (A) Representative myocardial sections stained for: left panels, wheat germ agglutinin (red), isolectin B4 (green) and DAPI (blue); and right panels, picrosirius red; (B,E) Gravimetric measurement of cardiac size (HW/TL, heart weight/tibia length); (C,F) Cardiomyocyte cross sectional area; $(\mathbf{D}, \mathbf{G})$ Capillary-to-myocyte ratio; $(\mathbf{H})$ Basal phosphorylation of AKT (Ser473); (I,J) Relative Mrna expression of genes associated with the physiological growth program. Data are represented as mean \pm SEM; ${ }^{*} p<0.05,{ }^{* *} p<0.01,{ }^{* * *} p<0.001,{ }^{* * * *} p<0.0001 ; n=6-12$ per group. 
FIGURE 18

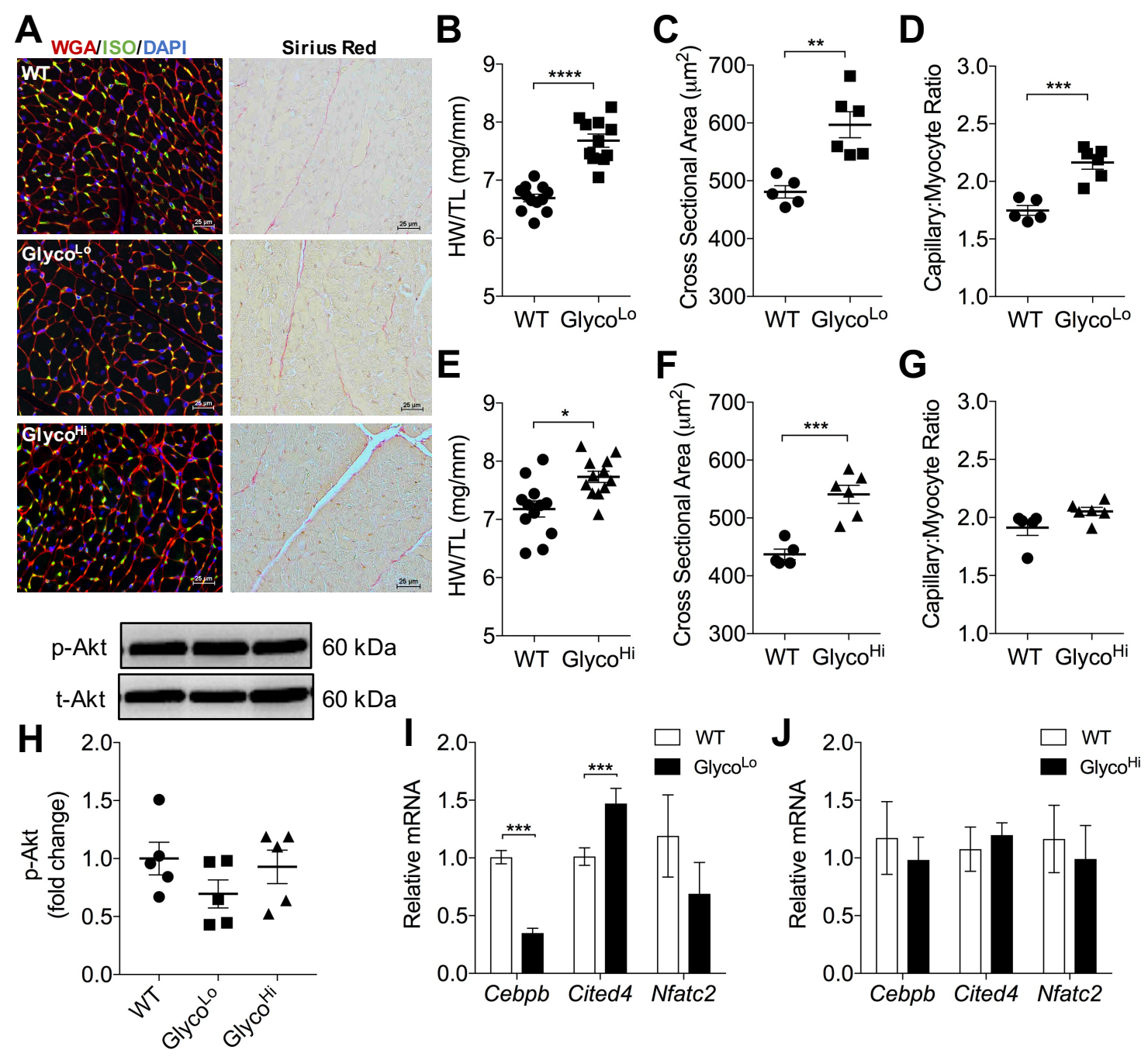


Table 7: Echocardiographic parameters from $\mathrm{Glyco}^{\mathrm{Lo}}$ and $\mathrm{Glyco}^{\mathrm{Hi}}$ mice compared with WT controls. Cardiac structure and function were evaluated in 20-week-old, male, cardiac-specific, kd-PFK2 mice (Glyco ${ }^{\text {Lo }}$ mice), pd-PFK2 mice $\left(\right.$ Glyco $^{\mathrm{HI}}$ mice) and WT littermates by echocardiography. Abbreviations: HR, heart rate; EDV, end-diastolic volume; ESV, end-systolic volume; SV, stroke volume; $E F$, ejection fraction; CO, cardiac output; LVIDd, left ventricular internal diameter in diastole; LVIDs, left ventricular internal diameter in systole; LVPWd, left ventricular posterior wall in diastole; LVPWs, left ventricular posterior wall in systole; LVAWd, left ventricular anterior wall in diastole; LVAWs, left ventricular anterior wall in systole; RWT, relative wall thickness. ${ }^{*} p<0.05,{ }^{* *} p<0.01$ vs. corresponding WT controls. 
TABLE 7

\begin{tabular}{|c|c|c|c|c|c|c|c|c|c|c|c|c|}
\hline & \multicolumn{3}{|c|}{ WT } & \multicolumn{3}{|c|}{ Glyco $^{\text {Lo }}$} & \multicolumn{3}{|c|}{ WT } & \multicolumn{3}{|c|}{$\mathrm{Glyco}^{\mathrm{Hi}}$} \\
\hline & Mean & & SD & Mean & & SD & Mean & & SD & Mean & & SD \\
\hline HR (bpm) & 486 & \pm & 22 & 486 & \pm & 26 & 492 & \pm & 52 & 494 & \pm & 38 \\
\hline \multicolumn{13}{|l|}{ Endocardial Values } \\
\hline $\operatorname{EDV}(\mu \mathrm{l})$ & 44 & \pm & 4 & 49 & \pm & $5 *$ & 50 & \pm & 4 & 54 & \pm & 7 \\
\hline $\operatorname{ESV}(\mu \mathrm{l})$ & 12 & \pm & 3 & 15 & \pm & 4 & 15 & \pm & 2 & 20 & \pm & $5 * *$ \\
\hline $\mathrm{SV}(\mu \mathrm{l})$ & 32 & \pm & 3 & 34 & \pm & 3 & 35 & \pm & 4 & 34 & \pm & 4 \\
\hline EF (\%) & 72 & \pm & 4 & 70 & \pm & 6 & 70 & \pm & 4 & 63 & \pm & $5 * *$ \\
\hline $\mathrm{CO}(\mathrm{ml} / \mathrm{min})$ & 15 & \pm & 2 & 16 & \pm & 2 & 17 & \pm & 2 & 17 & \pm & 2 \\
\hline \multicolumn{13}{|l|}{ Chamber Diameter } \\
\hline LVIDd (mm) & 3.9 & \pm & 0.2 & 4.0 & \pm & 0.3 & 4.1 & \pm & 0.2 & 4.4 & \pm & $0.3^{*}$ \\
\hline LVIDs (mm) & 2.3 & \pm & 0.3 & 2.3 & \pm & 0.3 & 2.5 & \pm & 0.3 & 2.9 & \pm & $0.3 * *$ \\
\hline \multicolumn{13}{|l|}{ Wall Thickness } \\
\hline LVPWd (mm) & 0.8 & \pm & 0.1 & 0.9 & \pm & $0.1 *$ & 1.0 & \pm & 0.2 & 1.0 & \pm & 0.1 \\
\hline LVPWs (mm) & 1.3 & \pm & 0.2 & 1.4 & \pm & 0.2 & 1.5 & \pm & 0.2 & 1.4 & \pm & 0.1 \\
\hline LVAWd (mm) & 0.9 & \pm & 0.1 & 0.9 & \pm & 0.1 & 1.1 & \pm & 0.2 & 1.2 & \pm & 0.2 \\
\hline LVAWs (mm) & 1.3 & \pm & 0.2 & 1.3 & \pm & 0.2 & 1.6 & \pm & 0.3 & 1.6 & \pm & 0.2 \\
\hline RWT & 0.43 & \pm & 0.07 & 0.47 & \pm & $0.10^{*}$ & 0.51 & \pm & 0.10 & 0.45 & \pm & 0.07 \\
\hline$(\mathrm{n}=)$ & \multicolumn{3}{|c|}{10} & \multicolumn{3}{|c|}{10} & \multicolumn{3}{|c|}{11} & \multicolumn{3}{|c|}{11} \\
\hline
\end{tabular}


elevated ESV, lower EF\%, and larger chamber diameters, suggesting a more dilated cardiac phenotype (Table S5).

To determine whether the signaling mediators and transcriptional programs underlying exercise-induced cardiac growth ${ }^{43,45,46,218}$ are affected by constitutive changes in glucose utilization, we next examined the phosphorylation of AKT and the relative expression of Cebpb and Cited4. While no differences in basal p-AKT (S473) were observed in either strain (Fig. 18H), Cebpb expression was lower and Cited4 expression was higher in Glyco ${ }^{\text {Lo }}$ hearts compared with WT counterparts (Fig. 18I). Expression of these genes was unchanged in $\mathrm{Glyco}^{\mathrm{Hi}}$ hearts (Fig. 18J). Although Nfatc2 showed a trend toward lower expression in Glyco ${ }^{\text {Lo }}$ hearts, this did not reach statistical significance. These data suggest that constitutively low or high rates of glycolysis cause mild cardiac hypertrophy and that a low rate of glycolysis, in the absence of AKT activation, is sufficient to promote a structural, functional, and transcriptional phenotype resembling the exercise-adapted heart.

Metabolic inflexibility causes mitochondrial structural and functional abnormalities. Because constitutive polarization of metabolism toward either glucose or fat oxidation could have deleterious effects on mitochondrial structure and function, we examined mitochondrial ultrastructure by transmission electron microscopy and measured respiration in mitochondria isolated from the hearts of both Glyco ${ }^{\text {Lo }}$ and $\mathrm{GlycO}^{\mathrm{Hi}}$ mice. As shown in Fig. 19A, qualitative analysis of electron micrographs showed disorganization of mitochondrial cristae and diminished cristae density compared with WT littermates. Consistent with this observation, 
mitochondria from both $\mathrm{Glyco}^{\mathrm{LO}}$ and $\mathrm{Glyco}^{\mathrm{Hi}}$ hearts showed lower respiration in the presence of pyruvate+malate or succinate (Fig. 19B,C). In addition, respiratory control ratios (RCR) were found to be lower in cardiac mitochondria from both transgenic strains, suggesting diminished coupling of mitochondrial electron transport to ATP synthesis (Fig. 19D). Thus, while constitutively low glycolysis imparts some structural, functional, and transcriptional features characteristic of the exercise-adapted heart, the mitochondrial compartment is damaged by metabolic inflexibility.

Constitutively low glycolysis is sufficient for maximal cardiac growth. With the observation that Glyco ${ }^{\mathrm{Lo}}$ hearts have a cardiac phenotype partially resembling the exercise-adapted heart, we next asked whether these mice are capable of performing more work or adapting to a greater degree than their WT counterparts. To address this question, we subjected both WT and Glyco ${ }^{\text {Lo }}$ mice to exercise testing prior to and following 4 weeks of treadmill training. Although $\mathrm{Glyco}^{\mathrm{LO}}$ mice were able to adapt systemically to the training regimen, they were not able to run farther or perform more work than WT mice (Fig. 20A,B). Unlike WT mice, which showed a $\sim 15 \%$ increase in cardiac size, Glyco ${ }^{\text {Lo }}$ mice showed no further increase in cardiac growth following training (Fig. 20C). Similarly, myocardial expression of Cebpb and Cited 4 were not found to be different in exercised Glyco ${ }^{\mathrm{LO}}$ mice compared with sedentary Glyco ${ }^{\mathrm{LO}}$ mice (Fig. 20D), suggesting that their baseline cardiac phenotype is sufficient to meet the increased cardiovascular demands brought about by exercise. These results suggest that constitutively low glycolytic 
Figure 19: Metabolic inflexibility disrupts cristae structure and causes mitochondrial dysfunction. (A) Electron micrographs of WT, Glyco ${ }^{\mathrm{LO}}$, and Glyco ${ }^{\mathrm{Hi}}$ hearts. Arrows indicate areas of decreased cristae organization and density compared to WT controls; (B) State 3 and (C) State 4 respiration in isolated cardiac mitochondria measured using complex I (Pyruvate+Malate)- and complex II (Succinate)-specific substrates; (D) Respiratory control ratios. Data are represented as mean $\pm \mathrm{SEM} ;{ }^{*} p<0.05,{ }^{* *} p<0.01 ; n=3$ per group. 
FIGURE 19

A
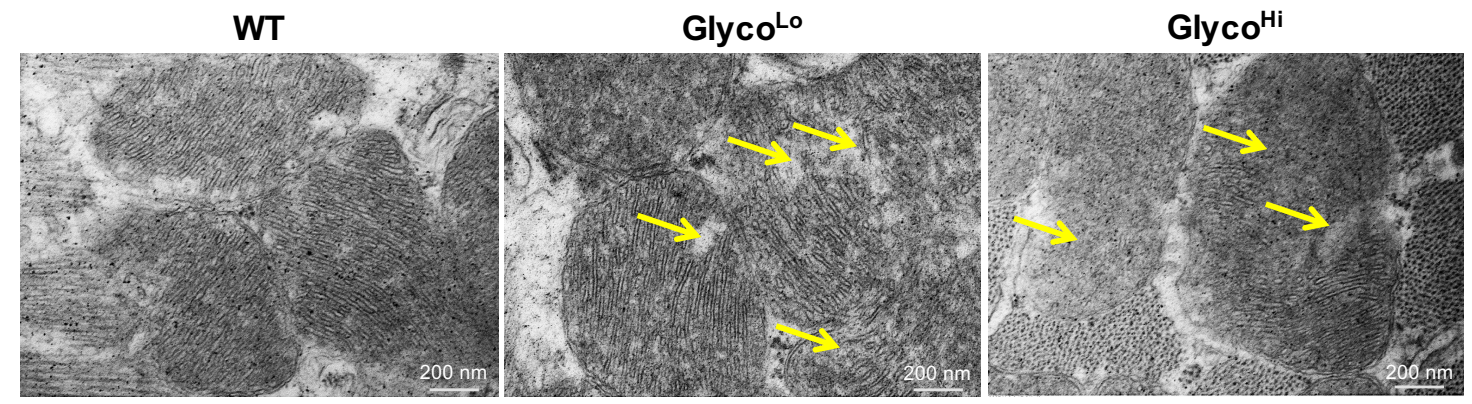

B

State 3

C

State 4
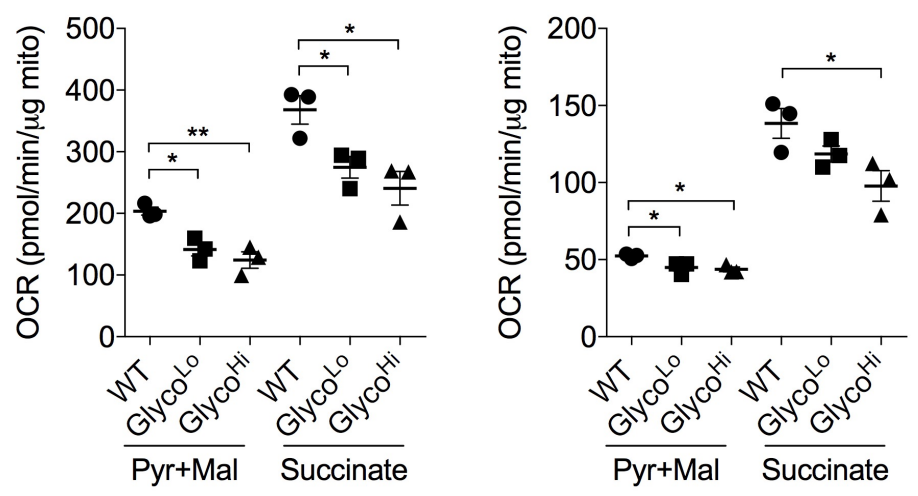

D

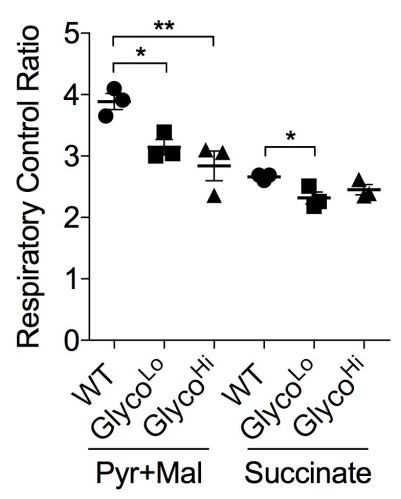


Figure 20: Low myocardial glycolytic rates are sufficient for maximal cardiac growth. Effects of exercise training on exercise capacity, cardiac growth, and mitochondrial function in WT and Glyco ${ }^{\mathrm{Lo}}$ mice: Pre- and post-training (A) distance and (B) work; (C) Percent cardiac growth in exercise-adapted (Exe) mice compared to sedentary (Sed) controls. $N=10-11$ per group; (D) Relative Mrna expression of genes associated with the physiological growth program. $\mathrm{N}=5 \mathrm{per}$ group; (E) State 3 respiration in isolated cardiac mitochondria using complex I (Pyruvate+Malate)-, complex II (Succinate)-, and fatty acid oxidation (Palmitoylcarnitine+malate)-specific substrates. $\mathrm{N}=3$ per group. Data are represented as mean $\pm \mathrm{SEM} ;{ }^{*} \mathrm{p}<0.05,{ }^{* * * *} \mathrm{p}<0.0001$. 
FIGURE 20

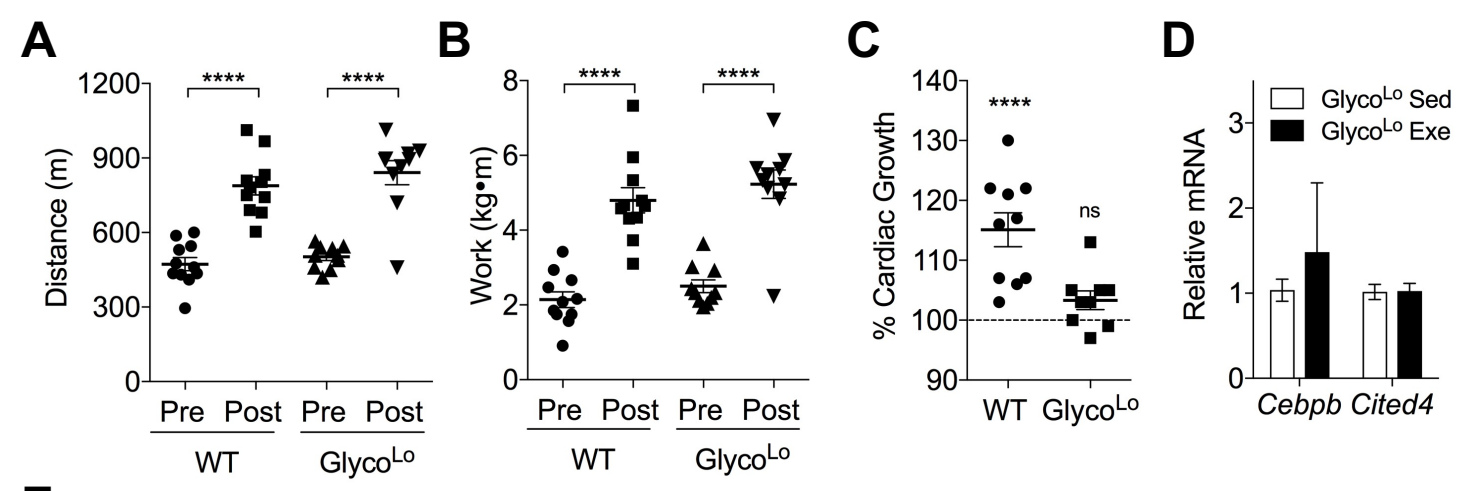

E
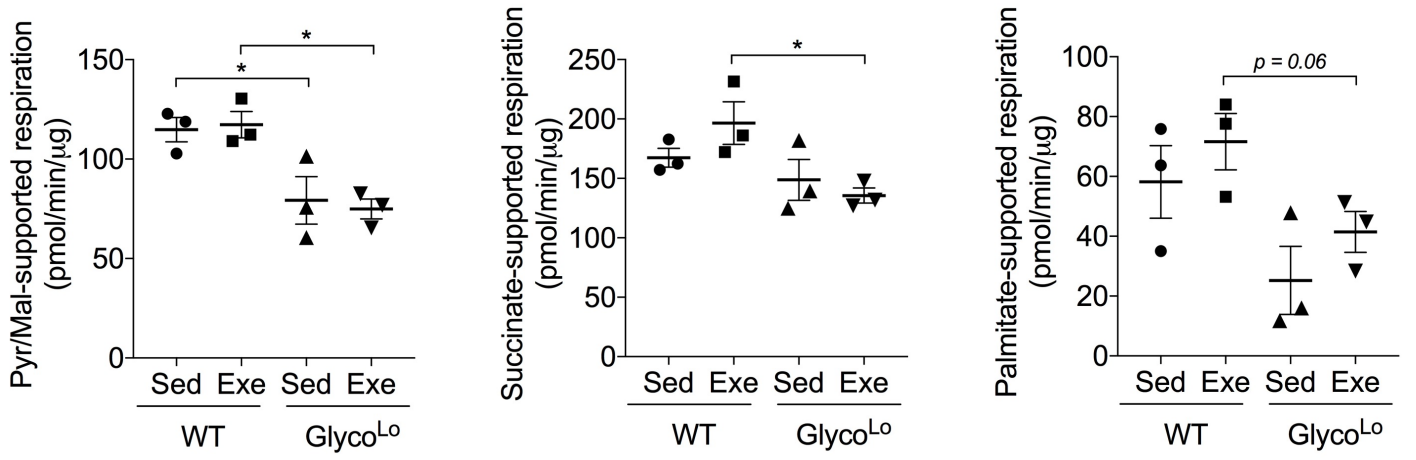
rate is sufficient to maximally activate physiologic cardiac growth.

Because exercise could have beneficial effects on mitochondrial function, ${ }^{39}$ we next posited that it might restore mitochondrial function in Glyco ${ }^{\text {Lo }}$ hearts. In WT mice, exercise did not affect state 3 respiration in the presence of any substrate tested, and, in Glyco ${ }^{\mathrm{LO}}$ mice, exercise did not reverse mitochondrial dysfunction caused by their metabolic phenotype (Fig. 20E).

Glycolysis regulates gene expression in the heart. To gain further insight into the extent to which glycolysis might regulate myocardial gene expression, we assessed relative transcript abundance in $\mathrm{Glyco}^{\mathrm{LO}}$ and $\mathrm{Glyco}^{\mathrm{Hi}}$ hearts. Results of Genechip ${ }^{\circledR}$ arrays showed that the expression of 2001 genes were significantly different in $\mathrm{Glyco}^{\mathrm{LO}}$ and $\mathrm{Glyco}^{\mathrm{Hi}}$ hearts compared with hearts of corresponding WT mice (Up: Glyco ${ }^{\mathrm{Lo}}, 7$; $\mathrm{Glyco}^{\mathrm{Hi}}, 309$; Down: Glyco ${ }^{\mathrm{Lo}}, 22$; Glyco ${ }^{\mathrm{Hi}}, 295$ genes); 14 differentially expressed genes overlapped in $\mathrm{Glyco}^{\mathrm{LO}}$ and $\mathrm{Glyco}^{\mathrm{Hi}}$ hearts). As shown in Fig. 21A, the top 100 genes include genes critical for: intermediary metabolism, neurotransmission and ion channel activity/transport, DNA damage/repair, cell proliferation/differentiation, cell signaling, gene transcription, inflammation and stress responses, detoxification and xenobiotic metabolism, and cytoskeletal/contractile function. We were particularly interested in genes that are differentially regulated by glycolysis, i.e., affected in diametric opposition with respect to low or high glycolytic activity (Fig. 21B). In addition to Cited4, we found that glycolysis regulates the expression of genes involved in one-carbon metabolism and downstream methylation reactions (Pah, Phgdh, Mtr), lipid 
Figure 21: Myocardial glycolysis coordinately regulates gene transcription in the heart. Results of transcriptomic analyses: (A) Robust Multi-array Average (RMA) analysis of top 100 genes that were found to be the most different based on the standard deviation across the samples; (B) Genes found to be coordinately regulated by glycolysis: Bars indicate genes with fold-changes at least 1.25-fold lower or higher in PFK2 mutant mice compared with WT controls. All genes shown were significantly different between $\mathrm{Glyco}^{\mathrm{LO}}$ and $\mathrm{Glyco}^{\mathrm{Hi}}$ mice at $p<0.05$, after adjustment for the false discovery rate. Significant differences from the WT controls (dotted line) using the same statistical analysis is shown by the asterisks ( ${ }^{*}$ FDR-adjusted p-value $<0.05$ ); (C) Gene ontology analysis of processes significantly regulated by low rates of glycolysis; and (D) Gene ontology analysis of processes significantly regulated by high rates of glycolysis. The 15 most significant processes are shown. $n=3$ per group. 
FIGURE 21

A

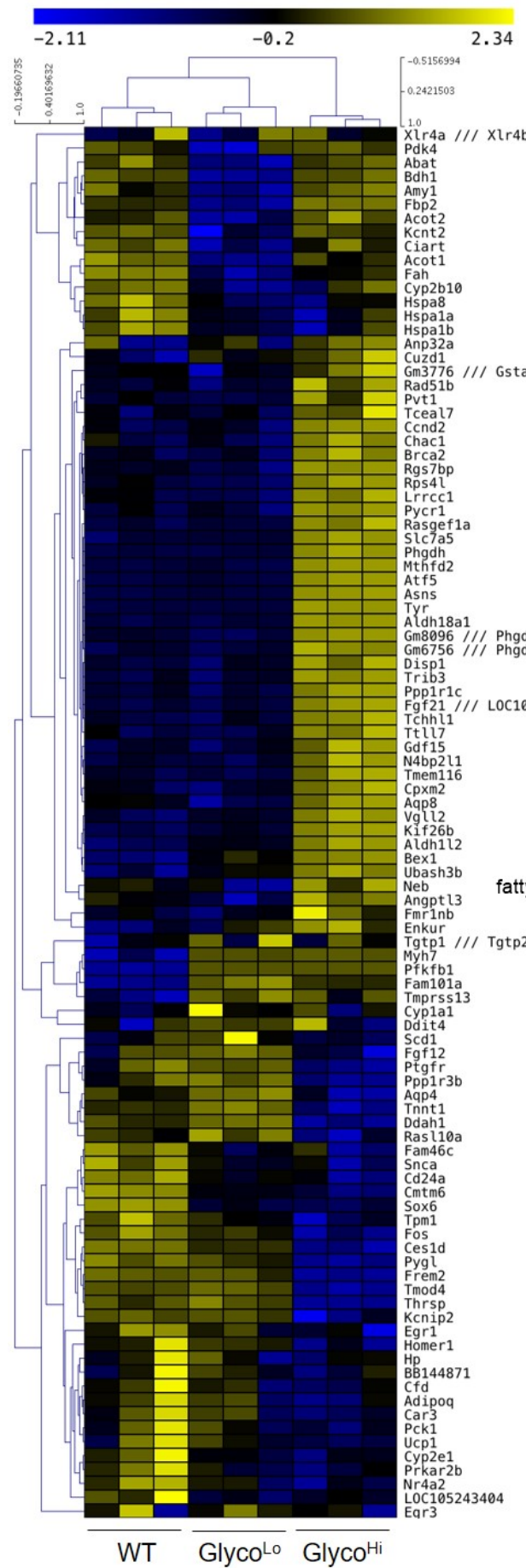

B

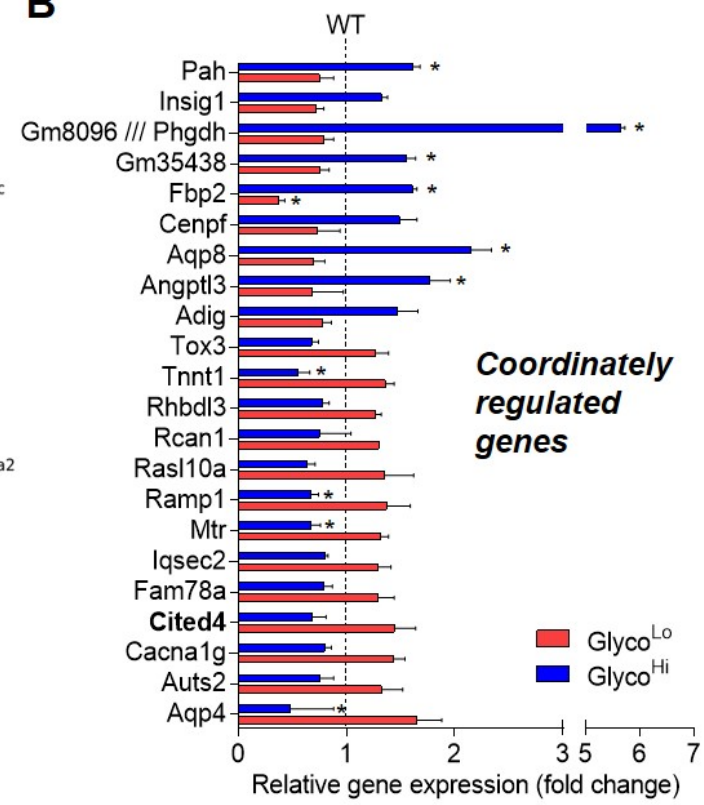

C
oxidation-reduction process response to CAMP
fatty acid beta-oxidation lipid homeostasis
response to stilbenoid
lipid metabolic process

fatty acid beta-oxidation using acyl-CoA dehydrogenase

long-chain fatty acid metabolic process

very long-chain fatty acid metabolic process

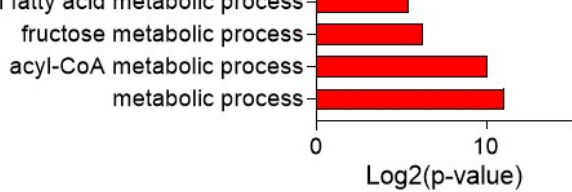

D

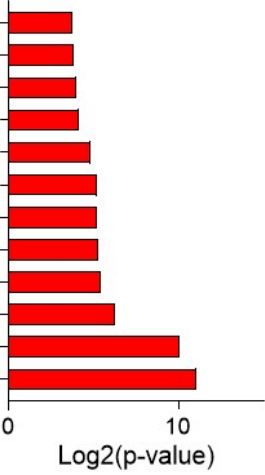

response to drug glutathione metabolic process pos. reg. of peptidyl-tyrosine phosphorylationcellular response to hormone stimulus pos. reg. of transcription, DNA-templated

fat cell differentiation angiogenesis phosphorylation

cellular amino acid biosynthetic process cellular response to extracellular stimulus neg. reg. of transcription from RNA pol II promoterresponse to CAMP oxidation-reduction proces metabolic process tRNA aminoacylation for protein translation

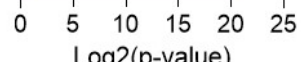


metabolism (Insg1, Angpt/3), gluconeogenic activity (Fbp2), cell division (Cenpf), osmoregulation (Cenpf, Aqp4), transcription (Tox3), G-protein signaling (Ramp1), calcium-related processes (Rcan1, Cacna1g). Gene Ontology analysis showed that low rates of glycolysis had a strong influence on genes involved in in oxidationreduction processes, lipid metabolism, and responses to cAMP (Fig. 21C). High glycolytic rates also influenced genes involved in metabolism, responses to cAMP, responses to hormonal stimuli, and transcription. Additionally, genes involved in angiogenesis as well as differentiation showed highly significant changes (Fig. 21D). Collectively, the findings of this study indicate that the activity of phosphofructokinase has pervasive effects on the metabolic, transcriptional, structural, and functional characteristics of the adult heart.

\section{$\underline{\text { Discussion }}$}

In this study, we provide evidence suggesting that exercise dynamically regulates myocardial glycolytic activity at the level of phosphofructokinase. To assess the importance of exercise-induced periodicity in glucose metabolism, we used transgenic mice expressing mutant transgenes that constitutively increase or decrease phosphofructokinase activity in the heart. Expression of a dominantnegative form of PFK2 (Glyco $\left.{ }^{\text {Lo }}\right)$ in the heart decreased glycolytic rate and was sufficient to modulate the Cebpb/Cited4 transcriptional program that regulates

physiologic cardiac growth. ${ }^{45,46,219}$ Mice in which myocardial glycolytic rate is constitutively high also showed slight hypertrophy, but these hearts did not engage the physiologic growth gene program. The corollary of these findings is that 
exercise-induced changes in phosphofructokinase activity are necessary to activate transcriptional programs dictating cardiac growth and hypertrophy. This is supported by our transcriptomics data from both $\mathrm{Glyco}^{\mathrm{Lo}}$ mice and $\mathrm{Glyco}^{\mathrm{Hi}}$ mice, which revealed coordinated regulation of not only the Cited 4 gene, but genes important for epigenetic regulation, lipid metabolism, gluconeogenic activity, contractility, and angiogenesis as well. Nevertheless, constitutive changes in myocardial metabolism incited damage to the mitochondrial compartment. Collectively, these results suggest that episodic decreases in glycolytic activity caused by exercise are required for physiologic cardiac remodeling and that metabolic flexibility is important to maintain mitochondrial health in the heart.

Our findings in the heart are reminiscent of exercise-induced changes in skeletal muscle, where transient and repetitive changes in gene expression, likely initiated by acute disruption of metabolic equanimity during exercise, stimulate adaptation. ${ }^{8}$ Although testing the role of metabolic periodicity in the heart is difficult, the post-translational regulation of PFK2 that we found to occur with exercise provided a unique opportunity to test not only how glucose utilization affects the cardiac phenotype, but how restricting fuel flexibility regulates cardiac structure, function, and gene expression as well. The dominant-negative approach to overcome the activity of endogenous myocardial PFK2, i.e., expression of the kinase-deficient form of PFK2, was sufficient to trigger a physiologic cardiac growth phenotype: Glyco ${ }^{\text {Lo }}$ hearts showed lower Cebpb and higher Cited4 expression, which mirrored the transcriptional changes found in the exercise-adapted heart. This transcriptional signature was complemented by a cardiac growth phenotype 
replete with enlarged myocytes and increased capillary density as well as cardiac chamber volumes, wall thicknesses, and functional values that were nearly identical to that found in the exercise-adapted heart. Glyco ${ }^{\text {Lo }}$ hearts appear to have a maximal functional and structural physiologic growth phenotype because they could not further activate the physiologic growth gene program or undergo additional cardiac growth in response to the exercise regimen. Importantly, the phenotype of Glyco $^{\text {Lo }}$ hearts developed in the absence of AKT activation, which has been suggested to underlie exercise-induced downregulation of Cebpb and upregulation of Cited4 and the general cardiac growth phenotype. ${ }^{27,} 218$ Thus, these data help form a model in which exercise-induced modulation of glucose metabolism is a proximal regulator of physiologic cardiac growth.

Several factors appear to contribute to acute, exercise-induced decreases in glycolysis and phosphofructokinase activity. The lower circulating blood glucose levels and increased levels of blood lactate and free fatty acids that we found to occur immediately after a single bout of exercise suggest diminished glycolytic activity occurring in the heart during and in the early recovery period after exercise. Indeed, lactate and fatty acids are preferred substrates of the heart that suppress glucose utilization both in vivo ${ }^{75,77,84}$ and ex vivo. ${ }^{78,79}$ Moreover, previous studies in humans and animal models suggest that changes in these substrates during exercise contribute to diminished exercise oxygen extraction ratios for glucose and lower glucose uptake and utilization. ${ }^{87,109}$ Not only do increases in circulating lactate and fatty acids with exercise appear to favor lower rates of glucose utilization, they divert glucose from glycolysis to glycogen synthesis, which is 
thought to be caused in part by diminished phosphofructokinase activity. ${ }^{86,130,240}$ Consistent with this idea, our results show diminished phosphorylation of S483 of PFK2 and increased levels of glycogen, which support the hypothesis that PFK1 activity and glycolysis are diminished with prolonged exercise or during the early recovery period. In the context of glucose utilization, changes in PFK1 are particularly important because this node of metabolism represents the major control point of glycolysis, exerting $65 \%$ control over flux through the pathway. ${ }^{235}$

Although constitutively low glycolytic rates appeared sufficient to drive physiologic growth, metabolic inflexibility in both the $\mathrm{Glyco}^{\mathrm{Hi}}$ and $\mathrm{Glyco}^{\mathrm{Lo}}$ hearts appeared sufficient to cause some pathology as well. While Glyco ${ }^{\mathrm{Hi}}$ hearts were enlarged, they did not demonstrate significantly increased capillary-to-myocyte ratios; and, functionally, they presented with a mildly diminished ejection fraction, significantly higher end systolic volume, and larger chamber diameters. These findings are in agreement with previous studies ${ }^{221}$ and suggest that chronically high rates of glycolysis promote a more pathological form of hypertrophy. Furthermore, that mitochondria isolated from both $\mathrm{Glyco}^{\mathrm{Hi}}$ and $\mathrm{Glyco}^{\mathrm{Lo}}$ hearts showed mitochondrial dysfunction suggests that deleterious changes in mitochondria occurring in the heart in the context of type II diabetes or heart failure — conditions associated with excessive myocardial reliance on fatty acids or glucose, respectively ${ }^{88,241}$ —could be in part caused by severe metabolic inflexibility. Although genetic downregulation of Cebpb and upregulation of Cited4 protect against pathologic insults, ${ }^{45,}{ }^{46}$ mice with constitutively low glycolysis subjected to pressure overload display exacerbated pathological remodeling, ${ }^{242}$ 
which we suggest may be due to poor mitochondrial health caused by metabolic inflexibility.

Although at steady state the exercise-adapted heart showed higher glycolytic activity, we found relatively few changes in metabolite abundance. We speculate that higher myocardial metabolic flux in the absence of changes in the steady state abundance of metabolites may be an indication of a physiological form of metabolic remodeling that occurs in the exercise-adapted heart. However, it should be noted that our results contrast somewhat with a previous finding that showed modestly lower abundances of acylcarnitines and organic acids in hearts of female mice adapted to two months of voluntary wheel running. ${ }^{243}$ The discrepancy between these two studies could be due to sex differences, strain differences, differences in the training modality and/or intensity. Additionally, these differences could be due to different methods by which hearts are handled prior to freezing for metabolomics analysis, which is an important consideration given the high-energy utilization of mouse heart.

Although our data support the idea that PFK-mediated modulation of metabolism influences transcriptional programs relating to cardiac growth and remodeling, it is possible that fat utilization or lactate oxidation, which are regulated in concert with glucose metabolism, are also important triggers of cardiac adaptation. Future studies to determine how exercise acutely changes the cardiac metabolome could help answer whether changes in metabolites in each pathway during or shortly after exercise promote biofeedback that coordinates transcriptional programming. Moreover, identifying how exercise intensity and 
duration regulate cardiac metabolism and gene transcription would be important for optimizing cardiac adaptations and for further understanding links between metabolism and cardiac growth. Particularly intriguing is the question of how such changes in metabolism may regulate epigenetic modifications. In our study, we found that glycolysis regulated Phgdh, Mthfd2, and Mtr, which code for proteins critical for serine biosynthesis, one-carbon metabolism, and methylation reactions. This could be significant because Cited4 is epigenetically regulated: hypermethylation of Cited4-associated CpG islands causes lower expression of Cited4 in oligodendroglial tumors. ${ }^{244}$ While our data do not address this mechanism, they suggest that low glycolytic rates could prevent such methylation events, thereby permitting Cited 4 transcription, especially when appropriate stimuli are present.

In summary, our results indicate that low rates of glycolysis during or shortly after exercise regulate gene programs important for cardiac growth and that invariantly high rates of glycolysis promote a more pathological form of hypertrophy. While metabolic inflexibility is damaging to mitochondria, changes in glucose utilization due to regular exercise appear to be important to maintain mitochondrial health and to derive maximal cardiac benefits from physical activity. In addition, by integrating metabolomic and transcriptomic data, these studies provide new insights into how PFK-mediated changes in metabolism regulate gene expression in the heart. Additional work is needed to further disclose how exercisemediated changes in metabolism regulate gene transcription and tissue 
remodeling and how these changes could be targeted to maximize the beneficial cardiovascular effects of exercise." ii This chapter previously appeared as an article in the journal Circulation. The original
citation is as follows: Gibb AA, et al. Exercise-induced changes in glucose metabolism
promote physiologic cardiac growth. Circulation, 2017. 


\section{CHAPTER IV}

\section{INTEGRATION OF FLUX MEASUREMENTS TO RESOLVE CHANGES IN ANABOLIC AND CATBOLIC METABOLISM IN CARDIAC MYOCYTES}

\section{$\underline{\text { Introduction }}$}

Glycolysis is the central pathway of intermediary carbon metabolism. Not only does catabolism of glucose via glycolysis provide useable energy for the cell, but it contributes glycolytic intermediates that serve as branch-point metabolites for the entry of glucose-derived carbon into ancillary pathways of glucose metabolism. These pathways are required for de novo synthesis of cellular components. For example, the activity of the pentose phosphate pathway (PPP) is required for nucleotide biosynthesis and the maintenance of cellular redox state, whereas the hexosamine biosynthetic pathway (HBP) regulates posttranslational protein modifications, protein processing, and stress responses. The synthesis of glycero(phospho)lipids and serine are also dependent on the production of glycolytic intermediates. ${ }^{245}$ In the heart, the PPP is activated during hypertrophy ${ }^{132-}$ ${ }^{134}$ and heart failure, ${ }^{135}$ and modulating the activity of this pathway regulates the severity of cardiac pathology. ${ }^{136-141}$ Similarly, O-linked $\beta$ - $N$-acetylglucosamine (OGIcNAc)-modified proteins, which require synthesis of the sugar donor UDPGIcNAc via the HBP, ${ }^{147,246}$ are elevated in hypertrophied and failing hearts. ${ }^{223,247-}$ 
${ }^{249}$ Although less is known about the glycerolipid pathway (GLP), increased activity of this pathway may underlie pathologic cardiac hypertrophy. ${ }^{142}$ In addition, numerous studies have identified aberrant ancillary pathway behavior in the context of diabetes [e.g., as reviewed in ${ }^{250,251}$ ].

The activities of ancillary pathways of glucose metabolism are regulated in part through expression of their rate-limiting or committed-step enzymes, cofactor availability, allosterism, and/or post-translational modifications [e.g., ${ }^{252-258}$ ]. Flux through these pathways may be regulated by other means as well. Specific enzymatic reactions in the glycolytic pathway, especially the phosphofructokinase 1 (PFK1) reaction, can coordinate the activity of ancillary pathways in several cell types $^{143-146}$; however, the effects of PFK activity and glycolytic rate on biosynthetic pathway flux in the heart remain unclear. Understanding how PFK regulates ancillary biosynthetic pathway activity is important because its activity is modulated by increased work ex vivo, ${ }^{259,260}$ and catecholamines ${ }^{261}$ and pressure overload in vivo. ${ }^{262}$

In this study, we examined how PFK activity regulates biosynthetic pathways in cardiomyocytes. For this, we altered PFK1 activity by expressing mutant forms of phosphofructo-2-kinase/fructose-2,6-bisphosphatase (PFK2) in primary rat neonatal cardiomyocytes. This method of regulating glycolysis leverages the strong allosteric regulation of PFK1 by F-2,6-P ${ }^{184,220,221,263}$ and is relevant to mechanisms regulating cardiovascular homeostasis. For example, kinases known to be important for cardiac responses to stress ${ }^{27,264}$ are strong regulators of PFK2 activity. ${ }^{236,} 265-267$ By integrating radiometric assays, stable 
isotope tracing, and extracellular flux analyses, we demonstrate that the amount of glucose available for entry into ancillary pathways appears relatively high and that the PFK node of glycolysis exerts varying degrees of control over glucose incorporation in the end products of the PPP, the HBP, and the GLP by not only diverting glucose carbon into these pathways, but by regulating mitochondrial activity and cataplerosis as well.

\section{Experimental Procedures}

Materials. DMEM growth media, containing L-glutamine, D-glucose, and pyruvate, was purchased from US Biological (Swampscott, MA, USA). Insulin, transferrin and selenium (500x) were purchased from Lonza (Walkersville, MD, USA). Humulin R was from Eli Lilly (Indianapolis, IN, USA). All other reagents were from Sigma-Aldrich Corp. (St. Louis, MO, USA), unless indicated otherwise.

Rodent models. All procedures were approved by the University of Louisville Institutional Animal Care and Use Committee and were in accordance with $\mathrm{NIH}$ guidelines. The euthanasia procedures were consistent with the AVMA Guidelines for the Euthanasia of Animals. Neonatal rat cardiomyocytes (NRCMs) were isolated from 1- to 2-day-old Sprague-Dawley rats as previously described. ${ }^{149,268,}$ ${ }^{269}$ Culture media was changed to serum-free DMEM media $24 \mathrm{~h}$ prior to each experiment. 
Gene transfer and expression analysis. Replication-deficient adenoviral vectors were constructed and purified by Vector BioLabs (Malvern, PA, USA) using cDNA for mutated forms of rat liver 6-phosphofructo-2-kinase/Fru-2,6- $\mathrm{P}_{2}$ bisphosphatase (PFKFB1 isoform of PFK2). The enzyme was engineered to have single amino acid point mutations to foster a kinase deficient- (S32D and T55V; Glyco ${ }^{\mathrm{LO}}$ ) or phosphatase deficient- (S32A and H258A; Glyco ${ }^{\mathrm{Hi}}$ ) form of PFK2. ${ }^{220,} 221,270-272$ A $1.4 \mathrm{~kb}$ BamHI/Nhe1 fragment of a pLenti6-3×FLAG-pd-PFK2 plasmid was subcloned into a Dual-CCM(+) shuttle vector, which has dual CMV promoters to drive both green fluorescent protein (GFP) and the insert. The backbone of the adenoviral vector is type 5 (dE1/E3). An Ad-GFP control virus, in which GFP is driven by the CMV promoter, was also purchased from Vector BioLabs.

Cardiac myocytes were transduced with adenovirus for $4 \mathrm{~h}$ in fresh media, followed by PBS wash and addition of fresh media. For all viral treatments, a multiplicity of infection (MOI) of 50 was used. Functional expression of the target protein was confirmed by immunoblotting and eGFP fluorescence. For measuring protein abundance, cells were lysed in a common lysis buffer containing $20 \mathrm{mM}$ HEPES, $110 \mathrm{mM} \mathrm{KCl}, 1 \mathrm{mM}$ EDTA, 1\% NP-40, 0.1\% SDS, and phosphatase and protease inhibitors. Cell lysates were then centrifuged at 13,000 $\mathrm{g}$ for $20 \mathrm{~min}$ and the supernatant was collected. Protein was measured using the standard Lowry Assay (Bio-Rad Laboratories, Hercules, CA, USA) and separated by SDS-PAGE, electroblotted to PVDF membranes, and probed for PFKFB1 (Novus Biologicals, Littleton, CO, USA) according to manufacturer's protocol. A horseradish peroxidase-linked secondary antibody (Cell Signaling Technology, Danvers, MA, 
USA) was used to detect and visualize the protein band with a Typhoon 9400 variable mode imager (GE Healthcare, Chicago, IL, USA).

Radiolabeled glycolysis assay. The rate of glucose utilization was determined using $\left[5-{ }^{3} \mathrm{H}\right]$-glucose as previously described. ${ }^{224}, 273,274$ Briefly, after $48 \mathrm{~h}$ of transduction, the media was replaced with $2 \mathrm{ml}$ of fresh, serum-free media containing 5.5 or $25 \mathrm{mM}$ glucose with the addition of $2 \mu \mathrm{Ci} / \mathrm{ml}\left[5-{ }^{3} \mathrm{H}\right]$-glucose (Moravek Biochemicals, Brea, CA, USA). Following incubation for $3 \mathrm{~h}, 100 \mu \mathrm{l}$ of media was collected and added to $100 \mu \mathrm{l}$ of $0.2 \mathrm{M} \mathrm{HCl}$ in a microcentrifuge tube. This tube, with the tube cap removed, was placed in a scintillation vial containing $500 \mu \mathrm{l}$ of $\mathrm{dH}_{2} \mathrm{O}$ to allow for evaporation diffusion of $\left[{ }^{3} \mathrm{H}\right]_{2} \mathrm{O}$ in the microcentrifuge tubes into the scintillation vials. To account for incomplete equilibration of $\left[{ }^{3} \mathrm{H}\right]_{2} \mathrm{O}$ and background, known amounts $(\mu \mathrm{Ci})$ of $\left[5-{ }^{3} \mathrm{H}\right]$-glucose and $\left[{ }^{3} \mathrm{H}\right]_{2} \mathrm{O}$ (Moravek Biochemicals) were placed into microcentrifuge tubes, and these were placed into

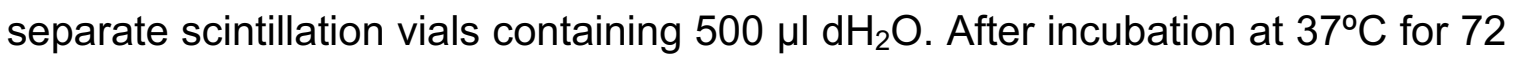
$\mathrm{h}$, the microcentrifuge tube was removed from the vial, $10 \mathrm{ml}$ of scintillation fluid was added, and scintillation counting was performed using a Tri-Carb 2900TR Liquid Scintillation Analyzer (Packard Bioscience Company, Meriden, CT, USA). Glucose utilization was then calculated as reported by Ashcroft et al., ${ }^{224}$ with considerations for the specific activity of $\left[5-{ }^{3} \mathrm{H}\right]$-glucose, incomplete equilibration and background, dilution of $\left[5-{ }^{3} \mathrm{H}\right]-$ to unlabeled-glucose, and scintillation counter efficiency. To normalize glucose utilization to total protein, the cells were first washed with PBS (to remove any adherent radioactivity) and then lysed in common 
lysis buffer. After centrifugation, protein concentration was measured using the Lowry assay kit (Bio-Rad Laboratories).

Stable Isotope Tracing. NRCMs were incubated in 6-well plates for $18 \mathrm{~h}$ in glucosefree DMEM media containing $1 \mathrm{mM}$ pyruvate and $4 \mathrm{mM}$ glutamine and supplemented with $25 \mathrm{mM}\left[{ }^{13} \mathrm{C}_{6}\right]$-glucose. Cell reactions were then quenched in cold acetonitrile, and extracted in acetonitrile:water:chloroform ( $\mathrm{v} / \mathrm{v} / \mathrm{v}, 2: 1.5: 1)$, as described previously, ${ }^{184,275-277}$ to obtain the polar, nonpolar, and insoluble proteinaceous fractions. The nonpolar (lipid) layer was collected, dried under a stream of nitrogen gas, and reconstituted in $0.1 \mathrm{ml}$ of chloroform:methanol:butylated hydroxytoluene $(2: 1+1 \mathrm{mM})$ mixture. The extract was diluted $10 \times$ with $1 \mathrm{mM}$ butylated hydroxytoluene solution in methanol and used for Fourier transform ion cyclotron resonance-mass spectrometry (FTICR-MS) analysis.

For stable isotope nucleotide analysis, the samples were prepared using a previously published protocol, ${ }^{184,}{ }^{277}$ with slight modifications. Briefly, lyophilized polar extracts were reconstituted in $50 \mu \mathrm{l}$ of $5 \mathrm{mM}$ aqueous hexylamine, adjusted

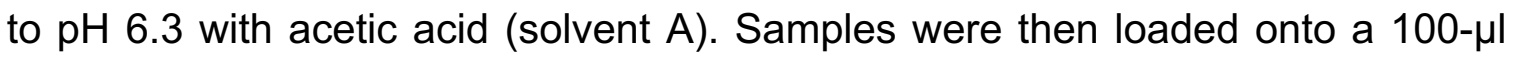
capacity C18 tip (Thermo Fisher Scientific, Waltham, MA, USA) followed by washing twice with $50 \mu \mathrm{l}$ of solvent $\mathrm{A}$. The metabolites were eluted with $70 \%$ solvent $\mathrm{A}$ and $30 \% 1 \mathrm{mM}$ ammonium acetate in $90 \%$ methanol, $\mathrm{pH} 8.5$ (solvent $\mathrm{B}$ ). The resulting eluates were diluted $3 \times$ with methanol and analyzed via FTICR-MS. 
$\left.{ }^{13} \mathrm{C}_{6}\right]$-glucose and $\left[{ }^{13} \mathrm{C}_{3}\right]$-lactate measurements in the media. For quantification of $\left[{ }^{13} \mathrm{C}_{6}\right]$-glucose and $\left[{ }^{13} \mathrm{C}_{3}\right]$-lactate in cell media, $10 \mu \mathrm{L}$ of media were mixed with $9990 \mu \mathrm{L}$ of $5 \mathrm{mM} \mathrm{NH} H_{4} \mathrm{OH}$ in methanol containing $5 \mu \mathrm{M}\left[\mathrm{D}_{2}\right]$-glucose and $5 \mu \mathrm{M}\left[\mathrm{D}_{2}\right]-$ lactate internal standard and used without any additional pre-treatment for FTICRMS analysis and quantification. Blank incubations without cells were run in parallel. The concentrations of $\left[{ }^{13} \mathrm{C}\right]$-labeled glucose and lactate were assessed using seven-point calibration curves $(0.3125 \mu \mathrm{M}$ to $20 \mu \mathrm{M})$.

FTICR-MS Analysis. Lipid and nucleotide spectra and $\left[{ }^{13} \mathrm{C}\right]$-glucose/lactate spectra were acquired using a hybrid linear ion trap-FT-ICR mass spectrometer (Finnigan LTQ FT; Thermo Electron, Bremen, Germany), equipped with a TriVersa NanoMate ion source (Advion Bio-Sciences, Ithaca, NY, USA) with an A electrospray chip (nozzle inner diameter, $5.5 \mu \mathrm{m}$ ). The TriVersa NanoMate was operated by applying $1.5 \mathrm{kV}$ with $0.5 \mathrm{psi}$ head pressure in positive ion mode and $1.6 \mathrm{kV}$ and $0.7 \mathrm{psi}$ in the negative mode. High mass accuracy data were collected using the FT-ICR analyzer over a mass range from 150 to $1600 \mathrm{Da}$ (lipids; + and - mode) for $15 \mathrm{~min}$ at the target mass resolution of 400,000 at $400 \mathrm{~m} / \mathrm{z}$. For quantification of ${ }^{13} \mathrm{C}_{6}$-glucose and ${ }^{13} \mathrm{C}_{3}$-lactate in cell media, data were collected in the negative ion mode for 2 min over a mass range from 50 to $250 \mathrm{Da}$ at the target mass resolution of 100,000 at $400 \mathrm{~m} / \mathrm{z}$. Prior to acquisition, the LTQ-FT was tuned and calibrated according to the manufacturer's default recommendations to achieve mass accuracy of $2 \mathrm{ppm}$ or less. 
Stable Isotope Data Analyses. The FT-ICR MS data were exported as exact mass lists into a spreadsheet file using Qual-Browser 2.0 (Thermo Electron). Isotopologue peak deconvolution, assignment, natural isotope abundance stripping, and quantification were performed using MetSign software, as described previously by us. ${ }^{184,278}$

Extracellular flux analysis of cellular energetics. To measure cellular energetics in intact cardiomyocytes, the Seahorse Bioscience XF24 extracellular flux analyzer was used as previously described. ${ }^{279}$ For this, 75,000 cells were plated into each well. An hour before each experiment, the media was replaced with $675 \mu$ l of assay media, i.e., unbuffered DMEM containing glucose $(5.5 \mathrm{mM})$, glutamine $(4 \mathrm{mM})$, and pyruvate $(1 \mathrm{mM}), \mathrm{pH} 7.4$. Following $1 \mathrm{~h}$ in a $37^{\circ} \mathrm{C}, \mathrm{CO}_{2}$-free incubator, the cells were placed in the instrument for analysis. Basal oxygen consumption rates (OCR) and extracellular acidification rates (ECAR) were measured using a programmed protocol: 3 cycles of 2 min mix, 2 min wait, and 3 min measure. To interrogate the effects of each treatment on cardiomyocyte bioenergetics, the following compounds were injected into each well with 2 cycles of 2 min mix, 2 min wait, 3 min measure following each injection: Port A, oligomycin (Oligo; $1 \mu \mathrm{M}$ ); Port $\mathrm{B}$, carbonyl cyanide-p-(trifluoromethoxy)phenylhydrazone (FCCP; $1 \mu \mathrm{M})$; and Port C, antimycin A and rotenone (AA/Rot; $10 \mu \mathrm{M} / 1 \mu \mathrm{M})$. The OCR and ECAR values were normalized to the total amount of protein in each well. 
Statistical Analysis. All values are mean \pm S.E.M. All comparisons were made to the relevant control group and performed using one-way analysis of variance (ANOVA) with Dunnett's correction for multiple comparisons. The null hypothesis was rejected when $p>0.05$.

\section{$\underline{\text { Results }}$}

PFK regulates glycolysis and the distribution of glucose. To understand the role of PFK activity in cardiac myocyte metabolism, we expressed kinase-deficient $\left(\mathrm{Glyco}^{\mathrm{LO}}\right)$ or phosphatase-deficient (Glyco $\left.{ }^{\mathrm{Hi}}\right)$ PFK2 mutant constructs in NRCMs, which are conducive to well-controlled and detailed metabolic flux analyses. The mutant genes were driven by a CMV promoter (Fig. 22A), and the bicistronic vector also contained GFP, used to determine relative transduction efficiency. As shown in Fig. 22B, the vast majority of NRCMs were transduced with virus. These PFK2 mutants were intentionally derived from the liver-specific isoform of PFK2 (i.e., PFKFB1) so that expression of the proteins could be assessed by immunoblotting, without obfuscation by other isoforms (e.g., PFKFB2/PFKFB3). Myocytes transduced with the Glyco ${ }^{\mathrm{LO}}$ or the $\mathrm{Glyco}^{\mathrm{Hi}}$ constructs showed strong bands at the predicted molecular mass for the mutant forms of PFK2, whereas cells transduced with Ad-GFP showed no expression of PFKFB1 (Fig. 22C). Although $\mathrm{Glyco}^{\mathrm{Hi}}$-expressing cells routinely displayed a higher level of mutant protein expression relative to Glyco ${ }^{\mathrm{LO}}$-expressing cells, the strength of the relative

effect of each PFK2 mutant on cellular glycolytic rate was equal, with a 2.1-fold increase and 2.2-fold decrease in glycolytic rate for the $\mathrm{Glyco}^{\mathrm{Hi}}$ and Glyco ${ }^{\mathrm{LO}}$ 
Figure 22: Phosphofructokinase activity regulates glucose metabolism in isolated cardiomyocytes. Glucose utilization analyses in neonatal rat cardiomyocytes: (A) Constructs of kinase-deficient and phosphatase-deficient PFK2 engineered to decrease $\left(\mathrm{Glyco}^{\mathrm{LO}}\right)$ or increase (Glyco ${ }^{\mathrm{Hi}}$ ) glycolytic rate, respectively; (B) GFP expression in cardiomyocytes $48 \mathrm{~h}$ after transduction (50 $\mathrm{MOI}$ ) with adenoviruses expressing GFP only or the $\mathrm{Glyco}^{\mathrm{LO}}$ or $\mathrm{Glyco}^{\mathrm{Hi}}$ adenoviruses; (C) Immunoblot of mutant PFK2 protein expression; (D) Glycolytic rate measured with $\left[5-{ }^{3} \mathrm{H}\right]$-glucose under 5.5 or $25 \mathrm{mM}$ glucose culture conditions. $\mathrm{n}=3-5$ independent isolations per group; $(\mathbf{E}){ }^{13} \mathrm{C}_{6}$-glucose uptake; $(\mathbf{F}){ }^{13} \mathrm{C}_{3}$-lactate production, and $(\mathbf{G})$ the percentage of glucose converted to lactate following $18 \mathrm{~h}$ incubation with isotopic glucose. Data in Panels $E-G$ represent three replicates per group. ${ }^{*} p<0.05,{ }^{* *} p<0.01,{ }^{* * *} p<0.001$. 
FIGURE 22

A

GlycoLo

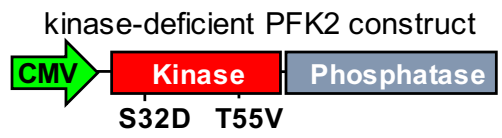

B

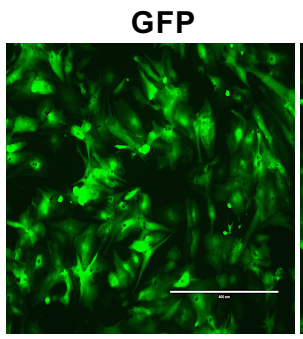

D

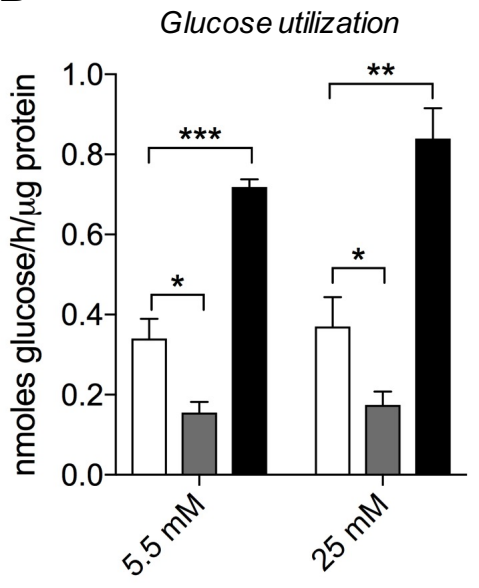

GlycoLo

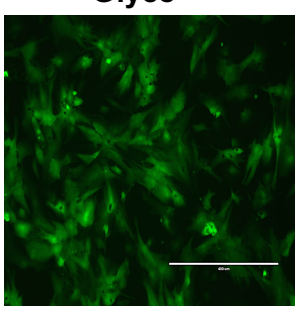

E

Glucose uptake
Glyco $^{H i}$

phosphatase-deficient PFK2 construct

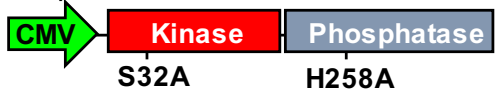

Glyco $^{\text {Hi }}$

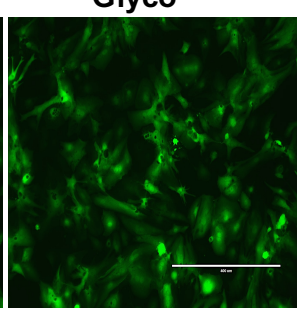

C

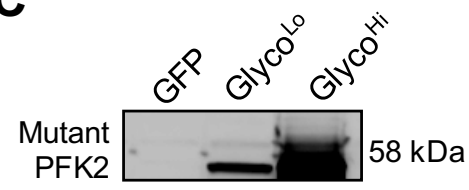

$\mathbf{F}$

Lactate production

G
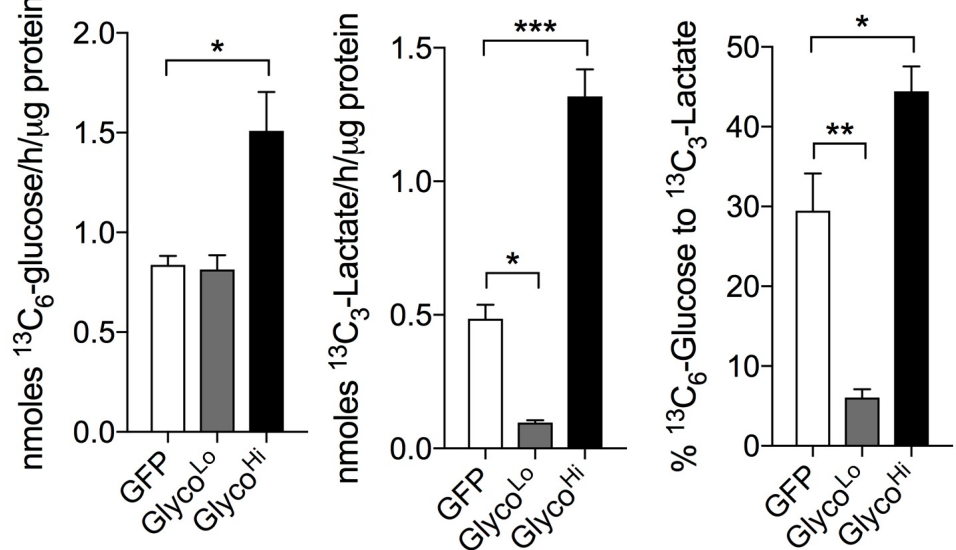
constructs, respectively (Fig. 22D). This effect on glycolytic rate was independent of glucose concentration, as rates were similar for groups incubated with either 5.5 or $25 \mathrm{mM}$ glucose.

To examine the effect of each mutant PFK2 enzyme on aerobic glycolysis, we replaced the normal growth media with media containing $\left[{ }^{13} \mathrm{C}_{6}\right]$-glucose and, after $18 \mathrm{~h}$, measured the relative levels of $\left[{ }^{13} \mathrm{C}_{6}\right]$-glucose and $\left[{ }^{13} \mathrm{C}_{3}\right]$-lactate in the media. As shown in Fig. 22E, the levels of $\left[{ }^{13} \mathrm{C}_{6}\right]$-glucose consumed by the cell was 1.8-fold higher in Glyco ${ }^{\mathrm{Hi}}$-expressing cells compared with Ad-GFP, with no difference in Glyco ${ }^{\text {Lo }}$-expressing cells. Levels of $\left[{ }^{13} \mathrm{C}_{3}\right]$-lactate extruded into the media was 2.5-fold higher in Glyco ${ }^{\mathrm{Hi}}$ cells compared with GFP control cells and $>10$-fold higher compared with Glyco ${ }^{\text {Lo }}$ virus transduced cells (Fig. 22F). As shown in Fig. 22G, 29\% of $\left[{ }^{13} \mathrm{C}_{6}\right]$-glucose was metabolized to $\left[{ }^{13} \mathrm{C}_{3}\right]$-lactate in GFP control cells, whereas in Glyco ${ }^{\mathrm{LO}}$-expressing cells, $6 \%$ of labeled glucose was metabolized to lactate; in Glyco ${ }^{\mathrm{Hi}}$-expressing cells, $44 \%$ of labeled glucose was converted to lactate.

Assessment of $\left[{ }^{13} \mathrm{C}_{6}\right]$-glucose uptake, $\left[{ }^{13} \mathrm{C}_{3}\right]$-lactate production, and $\left[5-{ }^{3} \mathrm{H}\right]$ glucose utilization provides estimates of not only how much glucose entered the cell and was later extruded as lactate, but also how much made it through the enolase step of glycolysis, where the tritiated label is eliminated as ${ }^{3} \mathrm{H}_{2} \mathrm{O}$. The mean values of glucose uptake and lactate production (provided by stable isotope tracing) and of radiometric measurements were used to calculate the amount of glucose unaccounted for by catabolism to pyruvate. In GFP control cardiomyocytes, only $44 \%$ of glucose was catabolized past the enolase step in 
glycolysis; of this fraction, the majority (65\%) was converted to lactate (Fig. 23A,B). These data show that, under control conditions, a substantial amount of glucose taken up by NRCMs is not catabolized to pyruvate and that the majority of glucose that is catabolized to pyruvate is fated for lactate production.

As shown in Fig. 1E, glucose uptake was not different between GFP and Glyco $^{\text {Lo }}$ cells, yet Glyco ${ }^{\text {Lo }}$ myocytes demonstrated 2-fold lower glucose utilization through the enolase step of glycolysis and 5-fold lower lactate production. In these cells, $78 \%$ of glucose-derived carbon was partitioned upstream of the enolase reaction, leaving 22\% available for pathways downstream of enolase (Fig. 23B). Although lactate production was markedly suppressed in Glyco ${ }^{\text {Lo }}$ cells, the absolute amount of glucose-derived carbon available for either mitochondrial oxidation, transamination to alanine, or carboxylation to oxaloacetate was identical to that of control cells. Interestingly, while Glyco ${ }^{\text {Hi }}$ cells showed $80 \%$ higher levels of glucose uptake compared with GFP control cells (see Fig. 1E), $44 \%$ of glucose (by mass) was not catabolized via glycolysis. Nevertheless, a portion of glucose equivalent to that apportioned upstream of enolase $(0.67 \mathrm{nmol} / \mathrm{h} / \mathrm{\mu g}$ protein $)$ taken into the cell could be accounted for as lactate $(0.66 \mathrm{nmol} / \mathrm{h} / \mathrm{\mu g}$ protein $)$, and $21 \%$ of glucose carbon that was catabolized past the enolase reaction was available for fates other than lactate production (Fig. 23B).

PFK activity regulates glucose carbon partitioning into the PPP in NRCMs. To determine how the activity of PFK regulates critical anabolic reactions derived from glycolysis, we modulated glycolytic rate by expressing the Glyco ${ }^{\mathrm{LO}}$ or Glyco ${ }^{\mathrm{Hi}} \mathrm{PFK} 2$ 
Figure 23. Phosphofructokinase-mediated glucose distribution in isolated cardiomyocytes. Schematics illustrating glucose utilization in myocytes transduced with GFP, Glyco ${ }^{\mathrm{LO}}$ or $\mathrm{Glyco}^{\mathrm{Hi}}$ adenoviruses. Measurements from radiometric and isotopic glucose utilization assays were used to delineate glucose distribution in neonatal rat cardiomyocytes transduced with each virus. (A) Schematic illustrating glucose distribution in control (GFP) myocytes. Distributions of glucose are drawn to relative scale. (B) Pie chart illustrating distribution of glucose: Numbers (in $\mathrm{nmol} / \mathrm{h} / \mathrm{\mu g}$ protein) and percentages (\%) indicate the absolute and relative distribution of glucose ascribed to the portions of glucose metabolism: upstream of enolase (light red), through enolase (grey shading), converted to lactate (yellow) and available for transamination to alanine, anaplerotic formation of oxaloacetate (OAA), or glucose oxidation (light blue). Pie charts are drawn to scale. Both schematics consider glucose uptake and lactate production provided by ${ }^{13} \mathrm{C}$-glucose tracing and by glucose utilization measured by radiometric assays, respectively. 
FIGURE 23
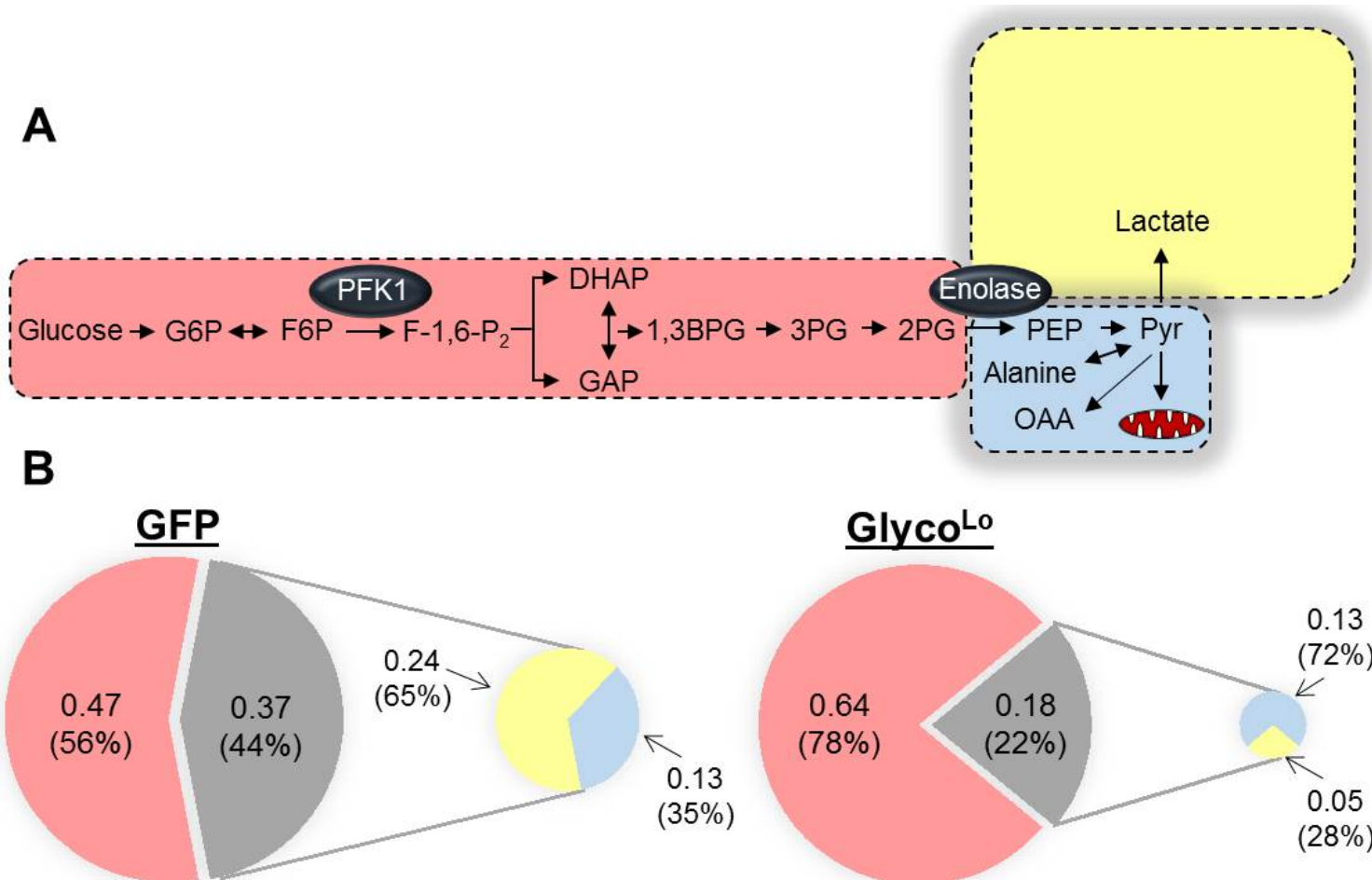

\section{GlycoLo}

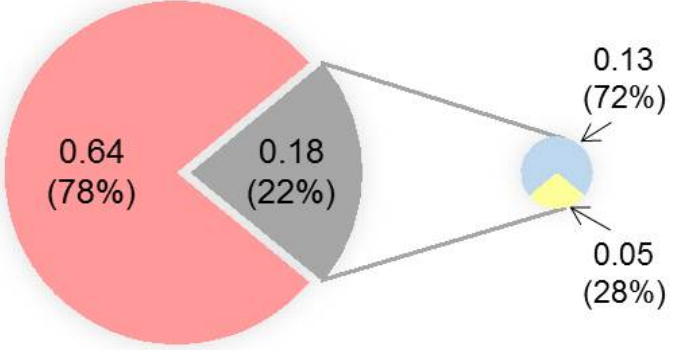

\section{Glyco $^{\mathrm{Hi}}$}

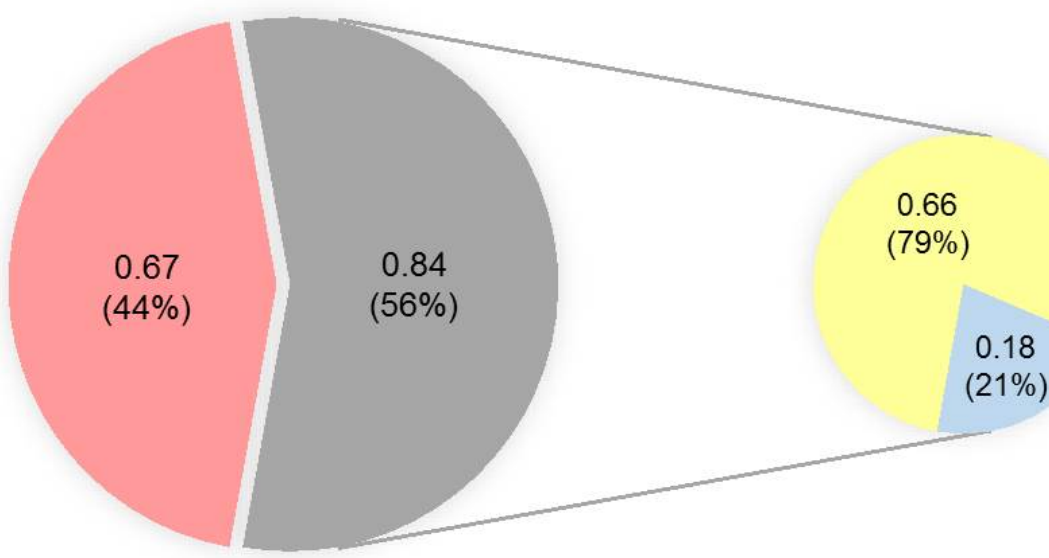

Above Enolase

Through Enolase

Converted to Lactate

Oxidation,

carboxylation, or transamination 
mutants in cardiomyocytes and then assessed carbon incorporation into end products of ancillary biosynthetic pathways using stable isotope-resolved metabolomics. ${ }^{280}$ Expression of the Glyco ${ }^{\mathrm{Hi}}$ mutant modestly diminished incorporation of glucose-derived carbon into ATP. Lower fractional enrichment of ${ }^{13} \mathrm{C}$ in $\mathrm{Glyco}^{\mathrm{Hi}}$-expressing cells is attributable to decreased carbon allocation into the phosphoribosyl ring of the nucleotide ( $m+5$ isotopologue) (Fig. 24A, 24B), suggesting diminished flux of ${ }^{13} \mathrm{C}$-labeled glucose-6-phosphate (G6P) through the oxidative PPP (oxPPP). The higher level of the $m+0$ isotopologue of ATP (i.e., the unlabeled ATP pool) in Glyco ${ }^{\mathrm{Hi}}$ cells further indicates that the flux of ${ }^{13} \mathrm{C}_{6}-\mathrm{G} 6 \mathrm{P}$ through the oxPPP was lower in Glyco ${ }^{\mathrm{Hi}}$ cells. Similar changes were observed in other purines such as GTP (Fig. 24C). Myocytes expressing the Glyco ${ }^{\mathrm{LO}}$ mutant showed ${ }^{13} \mathrm{C}$ fractional enrichment values into ATP that were similar to GFPtransduced control cells (Fig. 24A, 24B), suggesting that low PFK activity does not strongly affect purine synthesis in these cells.

For the pyrimidines CTP and UTP, we found a higher $m+0$ isotopologue value in Glyco ${ }^{\mathrm{Hi}}$ cells and a lower $m+0$ isotopologue value in Glyco ${ }^{\mathrm{LO}}$ cells compared with GFP controls. These data suggest that high rates of glycolysis diminish incorporation, and that low rates of glycolysis increase incorporation, of glucose-derived carbon into pyrimidines (Fig. 24D, 24E). Interestingly, both the Glyco ${ }^{\mathrm{LO}}$ and the Glyco ${ }^{\mathrm{Hi}}$ NRCMs showed increased levels of CTP and UTP having only the ribose ring labeled $(m+5$ isotopologue). These data could indicate that either decreasing or increasing glycolysis augments oxPPP flux. This interpretation would be consistent with the observation that both Glyco ${ }^{\mathrm{LO}}$ and 
Figure 24: Phosphofructokinase coordinates pentose phosphate pathway activity. Isolated cardiomyocytes were transduced with GFP, Glyco ${ }^{\mathrm{LO}}$, or Glyco ${ }^{\mathrm{Hi}}$ adenoviruses and incubated with ${ }^{13} \mathrm{C}_{6}$-glucose for $18 \mathrm{~h}$. Stable isotope tracing of nucleotides in NRCMs incubated with media containing U- ${ }^{13} \mathrm{C}$-glucose: (A) Atomresolved map illustrating the biological and biochemical history of ${ }^{13} \mathrm{C}$ incorporation into the purine AMP (phosphates in ATP omitted to conserve space); Fractional enrichment values of ${ }^{13} \mathrm{C}$ in (B) ATP and (C) GTP after incubation with ${ }^{13} \mathrm{C}$-glucose; (D) Atom-resolved map illustrating the biological and biochemical history of ${ }^{13} \mathrm{C}$ incorporation into the pyrimidine UMP; Fractional enrichment values of ${ }^{13} \mathrm{C}$ in (E) UTP and (F) CTP after incubation with ${ }^{13} \mathrm{C}$-glucose. Colored $\mathrm{x}$-axis labels correlate to the labeled molecule biosynthetic components indicated in the atom-resolved maps. Graph represents three replicates per group from one isolation. ${ }^{* \$} p<0.05$, ${ }^{* *, \$} p<0.01,{ }^{* * *, \$ \$} p<0.001,{ }^{* * * * \$ \$ \$} p<0.0001$. 
FIGURE 24

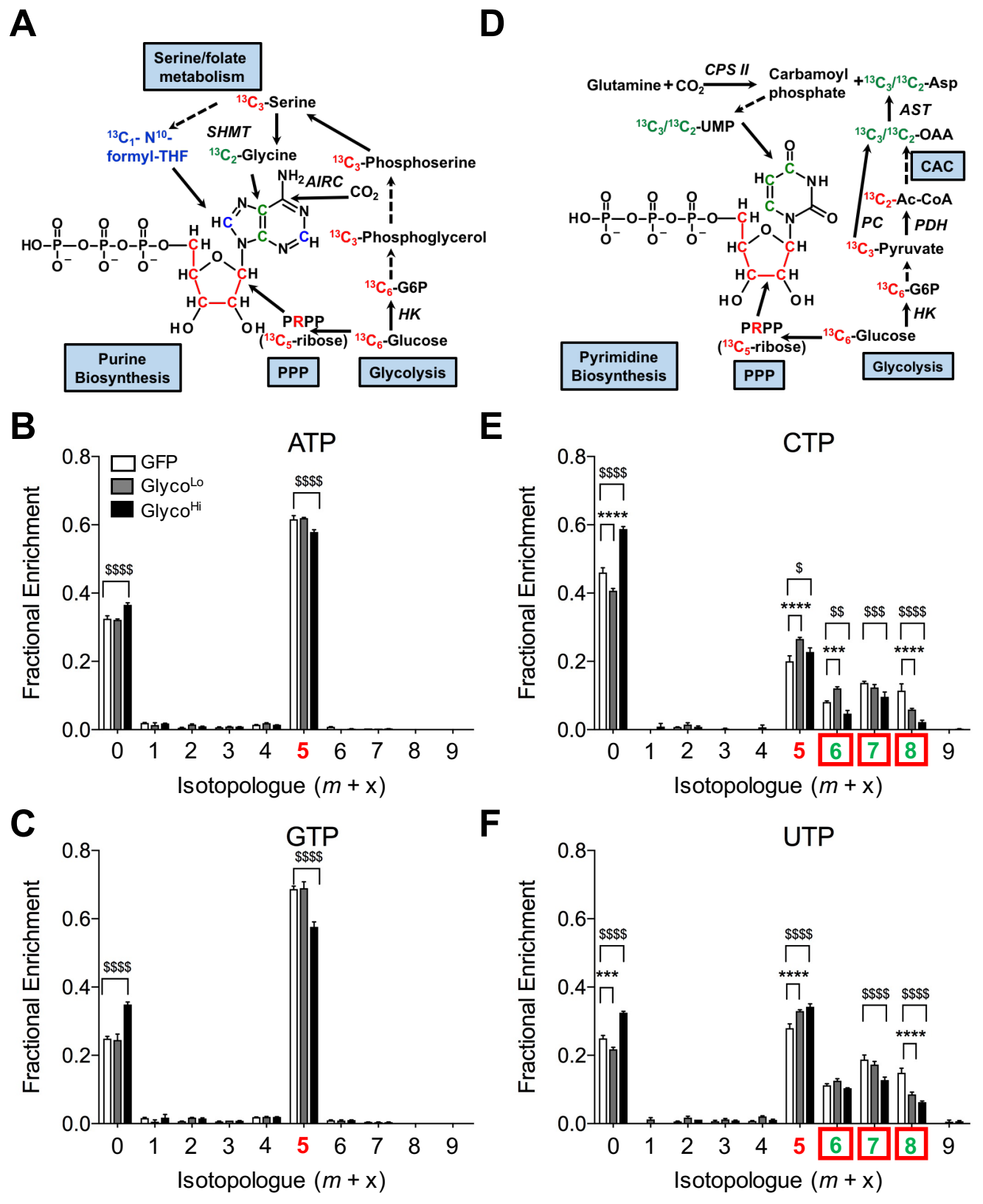


Glyco $^{\text {Hi }}$ cells show more glucose available for entry into ancillary biosynthetic pathways (see Fig. 2); however, it is also possible that higher levels of $m+5$ labeling could be due to an accumulation of these pyrimidine isotopologues, where the contribution of ${ }^{13} \mathrm{C}$-glucose to de novo synthesis of the uridine ring is diminished. Indeed, examination of the $m+7$ and $m+8$ isotopologues of UTP, which likely correspond to addition of carbons from ${ }^{13} \mathrm{C}$-labeled aspartate to the molecule (see Fig. 3D), show that both the Glyco ${ }^{\mathrm{LO}}$ and $\mathrm{Glyco}^{\mathrm{Hi}}$ cells have less incorporation of ${ }^{13} \mathrm{C}$, which is known to derive from Krebs cycle- $(m+7)$ or pyruvate carboxylaseengendered $(m+8)$ oxaloacetate. The $m+6$ isotopologue likely corresponds to complete ${ }^{13} \mathrm{C}_{5}$-labeling of the ribose ring and ${ }^{13} \mathrm{C}_{1}$ labeling of aspartate, occurring as a result of scrambling during the $2^{\text {nd }}$ turn of the Krebs cycle, as suggested previously. ${ }^{281}$ Overall, these data suggest that high PFK activity modestly diminishes oxPPP flux and ${ }^{13} \mathrm{C}$ incorporation into purines. Pyrimidine synthesis appears to be more robustly affected by PFK activity, with high rates of glycolysis diminishing incorporation of glucose-derived carbon into UTP and CTP molecules.

High PFK activity diminishes glucose-derived carbon incorporation into UDPHexNAc. The molecular synthesis of UDP-N-acetyl hexosamines requires end products or intermediates of several metabolic pathways, including those from the Krebs cycle, the pyrimidine biosynthetic pathway, glutamine, and glycolysis; hence, these pathways collectively contribute to UDP-HexNAc synthesis (Fig. 25A). To determine how the glycolytic activity affects glucose-derived carbon allocation into the HBP, we measured fractional enrichment of ${ }^{13} \mathrm{C}$ from $\left[{ }^{13} \mathrm{C}_{6}\right]-$ 
glucose into UDP-HexNAc in NRCMs transduced with $\mathrm{Glyco}^{\mathrm{Lo}}$ or Glyco ${ }^{\mathrm{Hi}}$ virus. After $18 \mathrm{~h},>90 \%$ of the UDP-HexNAc pool integrated ${ }^{13} \mathrm{C}$-glucose-derived carbon (Fig. 25B), which suggests that UDP-HexNAc has a faster rate of glucose carbon incorporation relative to purines and pyrimidines. That the $m+0$ isotopologue was higher in Glyco ${ }^{\mathrm{Hi}}$ cells indicates that high rates of glycolysis diminish glucose carbon incorporation into UDP-HexNAc. The lower ${ }^{13} \mathrm{C}$ enrichment of many isotopologues (e.g., $m+7, m+14, m+15, m+16$ ) appeared to be due to diminished input of metabolites that require mitochondrial transformation of ${ }^{13} \mathrm{C}$-glucosederived carbon; however, the $m+5, m+6$, and $m+11$ isotopologues (which are the forms of UDP-HexNAc with the ribose, hexose, and ribose+hexose moieties constituted with ${ }^{13} \mathrm{C}$, respectively) demonstrate significantly higher fractional enrichment in Glyco ${ }^{\mathrm{Hi}}$ cells. Given that both the $\mathrm{Glyco}^{\mathrm{Lo}}$ and the $\mathrm{Glyco}^{\mathrm{Hi}}$ cells had similar levels of glucose available for reactions upstream of enolase (see Fig. 2), the simplest explanation for this result is that the mitochondrial cataplerotic metabolites (deriving from the ${ }^{13} \mathrm{C}$-glucose-derived carbon source) are limiting for the synthesis of UDP-HexNAc; as a result, the $m+5$ and $m+6$ isotopologues of the metabolite accumulate as ${ }^{13} \mathrm{C}$ incorporation from cataplerotic metabolites slows down. This would be consistent with findings in pyrimidines, which suggest that cataplerotic generation of aspartate is limiting for the incorporation of glucosederived carbon into CTP and UTP.

Although low rates of glycolysis did not affect the overall level of incorporation of glucose-derived carbon into UDP-HexNAc, that the $m+11$ isotopologue in Glyco ${ }^{\mathrm{LO}}$ cells was higher than that in control cells suggests that low 
Figure 25: High phosphofructokinase activity diminishes glucose carbon incorporation into UDP-HexNAc. Isolated cardiomyocytes were transduced with GFP, Glyco ${ }^{\mathrm{Lo}}$, or Glyco ${ }^{\mathrm{Hi}}$ adenoviruses and incubated with ${ }^{13} \mathrm{C}_{6}$-glucose for $18 \mathrm{~h}$. Stable isotope tracing of UDP-HexNAc in NRCMs incubated with media containing ${ }^{13} \mathrm{C}$-glucose: (A) Atom-resolved map illustrating the biological and biochemical history of ${ }^{13} \mathrm{C}$ incorporation into UDP-GIcNAc; (B) Fractional enrichment values of ${ }^{13} \mathrm{C}$ in UDP-HexNAc (which includes both UDP-GIcNAc and UDP-GaINAc) after incubation with ${ }^{13} \mathrm{C}_{6}$-glucose Colored $\mathrm{x}$-axis labels correlate to the labeled molecule biosynthetic components indicated in the atom-resolved maps. Graph represents three replicates per group from one isolation. ${ }^{*} \$ p<0.05,{ }^{* *}, \$ \$ p<0.01,{ }^{* \$ \$}$ $p<0.001,{ }^{\$ \$ \$} p<0.0001$. 
FIGURE 25

A

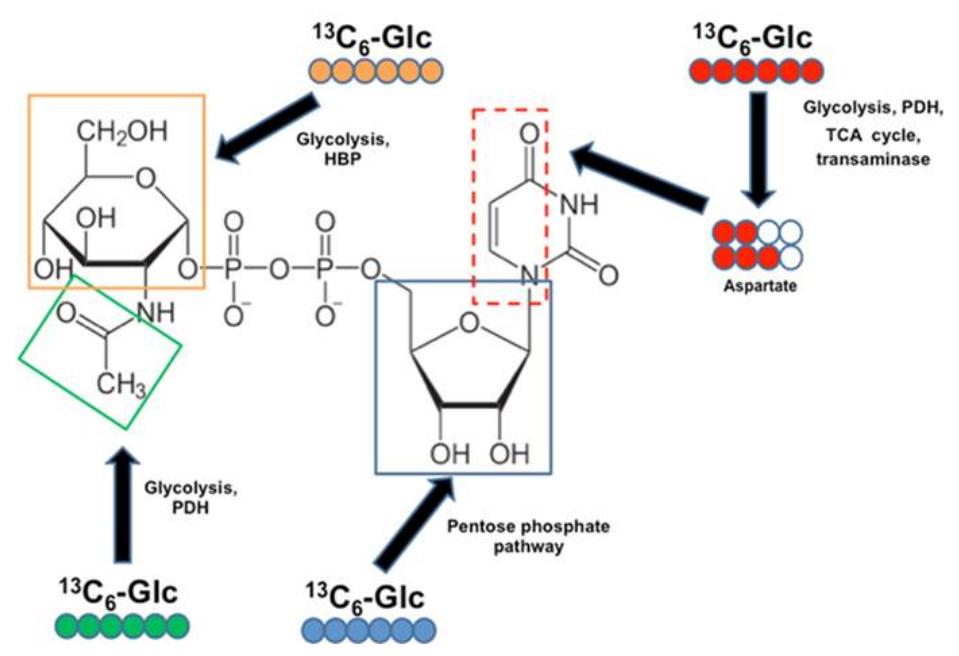

B

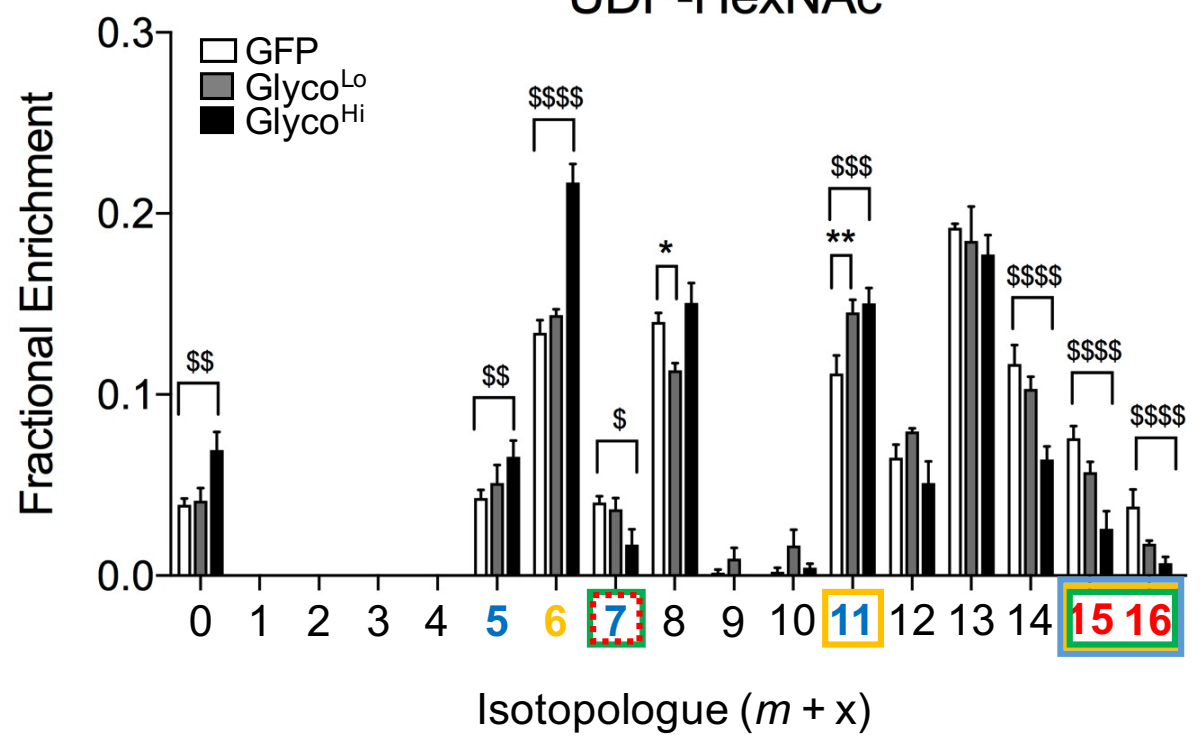


PFK activity promotes channeling of F6P not only into the HBP (contributing six ${ }^{13} \mathrm{C}$ ), but, because the ribose ring is included (contributing five ${ }^{13} \mathrm{C}$ ), the PPP as well (Fig. 25A, 25B). Changes in the $m+8$ isotopologue are difficult to interpret because this isotopologue could be comprised of ${ }^{13} \mathrm{C}$-labeled $\mathrm{N}$-acetyl hexosamine as well as stably labeled UDP. Altogether, these results suggest that high PFK activity decreases glucose carbon allocation into UDP-HexNAc primarily via limiting the mitochondria-derived and cataplerotic intermediates (e.g., acetyl CoA, aspartate) derived initially from ${ }^{13} \mathrm{C}$-glucose.

PFK regulates glycerolipid synthesis in cardiomyocytes. In cardiac myocytes, glycerolipid synthesis requires free fatty acids (FFAs) and glycerol-3-phosphate (G3P). While FFAs can be derived from transporter-mediated uptake across the plasma membrane ${ }^{282}$ myocytes have a relatively low capacity for free glycerol uptake; therefore, there is little contribution of glycerol kinase to G3P synthesis. ${ }^{283 \text {, }}$ 284 However, G3P can be generated from DHAP by glycerol-3-phosphate dehydrogenase (Fig. 26A). Nevertheless, it is unclear whether or how the rate of glycolysis affects glycerolipid synthesis. We found that in comparison with cells transduced with GFP virus, the Glyco ${ }^{\text {Lo }}$ cells showed diminished incorporation of ${ }^{13} \mathrm{C}$ from ${ }^{13} \mathrm{C}_{6}$-glucose into the glycerol $(m+3)$ moiety of phosphatidylcholine (PC;

Fig. 26B), phosphatidylinositol (PI; Fig. 26C), phosphatidylethanolamine (PE; Fig. 26D), and phosphatidylserine (PS; Fig. 26E). Incorporation of ${ }^{13} \mathrm{C}_{6}$-glucosederived carbon into fatty acids in PC, PI, PE, and PS was not different in Glyco ${ }^{\mathrm{Lo}}$ cells compared with control cells, suggesting that only the synthesis of the glycerol 
backbone portion of these phospholipids is affected by low rates of glycolysis.

High glycolytic rates affected ${ }^{13} \mathrm{C}$ incorporation into phospholipids to a lesser extent. Although PE synthesis showed a slight increase in ${ }^{13} \mathrm{C}$ fractional enrichment in glycerol (and a concomitant decrease in fractional enrichment of the unlabeled pool) (Fig. 26D), PI showed diminished labeling characterized by lower incorporation of ${ }^{13} \mathrm{C}$ into fatty acids. Glycerol ${ }^{13} \mathrm{C}$ labeling in PI from Glyco ${ }^{\mathrm{Hi}}$ cells was identical to that of control cells. This dichotomy in labeling patterns in different phospholipids could suggest the presence of unique enzyme complexes that participate in metabolic channeling for the synthesis of distinct glycerolipids. Consistent with this idea, triacylglycerol (TAG) labeling was remarkably different from that of other glycerolipids. As shown in Fig. 26F, TAG species collectively showed little ${ }^{13} \mathrm{C}$ in the glycerol moiety. Furthermore, low rates of glycolysis had little effect on glucose carbon incorporation into TAGs; however, Glyco ${ }^{\mathrm{Hi}}$ cells showed lower ${ }^{13} \mathrm{C}$ incorporation into fatty acids. Collectively, these results suggest that, in isolated NRCMs, low rates of glycolysis diminish phospholipid synthesis by the DHAP $\rightarrow$ G3P pathway and that high rates of glycolysis decrease incorporation of glucose-derived carbon into the fatty acyl chains of PI and TAGs.

PFK activity regulates mitochondrial activity in NRCMs: Stable isotope labeling patterns of several end products of the PPP, HBP, and GLP suggest that high PFK activity diminishes the relative abundance of those ${ }^{13} \mathrm{C}$ isotopologues that require metabolites derived from mitochondria. This suggests that cataplerosis ${ }^{285}$ is diminished under conditions of high PFK activity. Because cataplerotic reactions 
Figure 26: Phosphofructokinase regulates glycerolipid biosynthesis. Stable isotope tracing of phospholipids and triacylglycerols in cardiomyocytes incubated with media containing ${ }^{13} \mathrm{C}_{6}$-glucose for $18 \mathrm{~h}$ : (A) Atom-resolved map illustrating the biological and biochemical history of ${ }^{13} \mathrm{C}$ incorporation into glycerolipids; Fractional enrichment values of ${ }^{13} \mathrm{C}$ into: (B) phosphatidylcholine $(\mathrm{PC}) ; \quad$ (C) $\begin{array}{lllll}\text { Phosphatidylinositol } & \text { (PI); } & \text { (D) phosphatidylethanolamine } & \text { (PE); } & \text { (E) }\end{array}$ phosphatidylserine (PS); and (F) triacylglycerols (TAG). Graph represents three replicates per group from one isolation. ${ }^{*} \$ p<0.05,{ }^{* *, \$} p<0.01,{ }^{\$ \$} p<0.001,{ }^{\$ \$ \$}$ $p<0.0001$. 
FIGURE 26

A
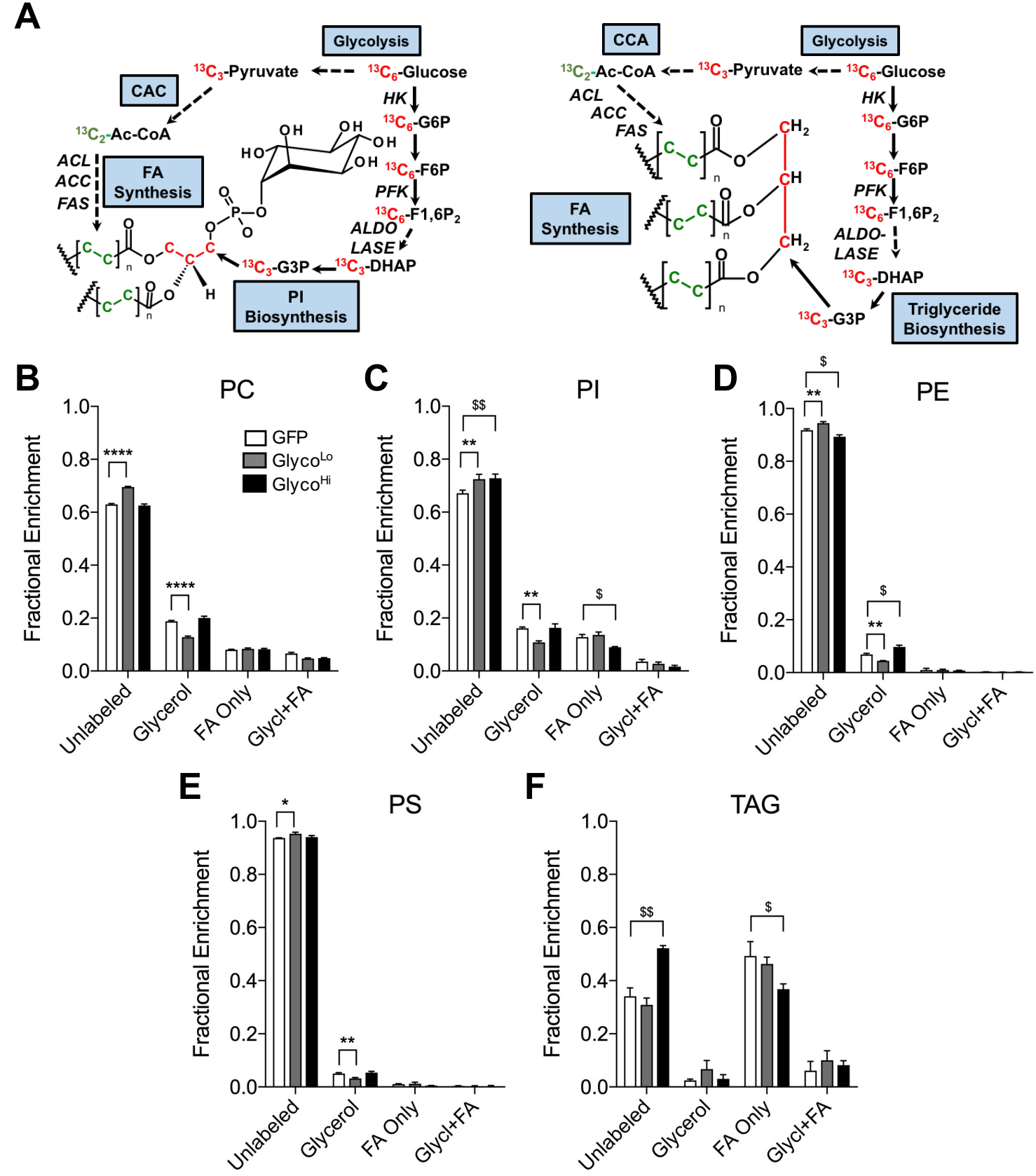
depend upon mitochondrial activity, we next measured respiration in cells expressing either the control construct (GFP) or the $\mathrm{Glyco}^{\mathrm{Hi}}$ or $\mathrm{Glyco}^{\mathrm{LO}}$ constructs. Glyco ${ }^{\mathrm{Hi}}$-expressing cells showed $50 \%$ lower basal oxygen consumption, whereas Glyco ${ }^{\text {Lo }}$-expressing cells showed significantly higher basal OCR values (Fig. 27A, 27C). These PFK-mediated changes in mitochondrial OCR are likely due to the Crabtree effect, which is characterized by decreases in mitochondrial activity under conditions of increased glucose availability or glycolytic activity. ${ }^{286-289}$ Although addition of oligomycin showed marginal effects on ATP-linked OCR, proton leak was significantly diminished in $\mathrm{Glyco}^{\mathrm{Hi}}$ cells. Upon addition of a mitochondrial uncoupler, Glyco ${ }^{\mathrm{LO}}$ and $\mathrm{Glyco}^{\mathrm{Hi}}$ cells showed relative changes in maximal respiratory rate and in reserve capacity that mirrored the pattern found for basal OCR and proton leak (Fig. 27A, 27C). These data are consistent with the notion that high PFK activity diminishes mitochondrial activity, which limits the cataplerotic generation of metabolites required for the synthesis of end products of the PPP, HBP, and GLP.

Although cells transduced with the Glyco ${ }^{\mathrm{Hi}}$ virus had $>2$-fold elevation in basal extracellular acidification (ECAR) compared with Ad-GFP controls, we observed no differences in ECAR in NRCMs expressing the Glyco ${ }^{\text {Lo }}$ PFK2 mutant (Fig. 27B). Addition of oligomycin increased ECAR to similar values in all groups, suggesting that inhibition of mitochondrial ATP production overrides PFK2mediated changes in glycolysis. Although extracellular acidification is a surrogate measure of lactate extrusion and aerobic glycolysis, protons are also generated during respiration via pyruvate dehydrogenase-mediated production of $\mathrm{CO}_{2}$ (i.e., 
Figure 27: Phosphofructokinase activity regulates mitochondrial activity in isolated cardiomyocytes. Extracellular flux analyses of NRCMs: (A) Oxygen consumption traces (OCR), (B) Extracellular acidification rate (ECAR), and (C) Calculated indices of respiration of cardiomyocytes transduced with GFP, Glyco ${ }^{\text {Lo }}$, or Glyco ${ }^{\mathrm{Hi}}$ adenoviruses. $\mathrm{n}=3$ independent isolations per group. ${ }^{*} \mathrm{p}<0.05,{ }^{* *} \mathrm{p}<$ 0.01 . 
FIGURE 27

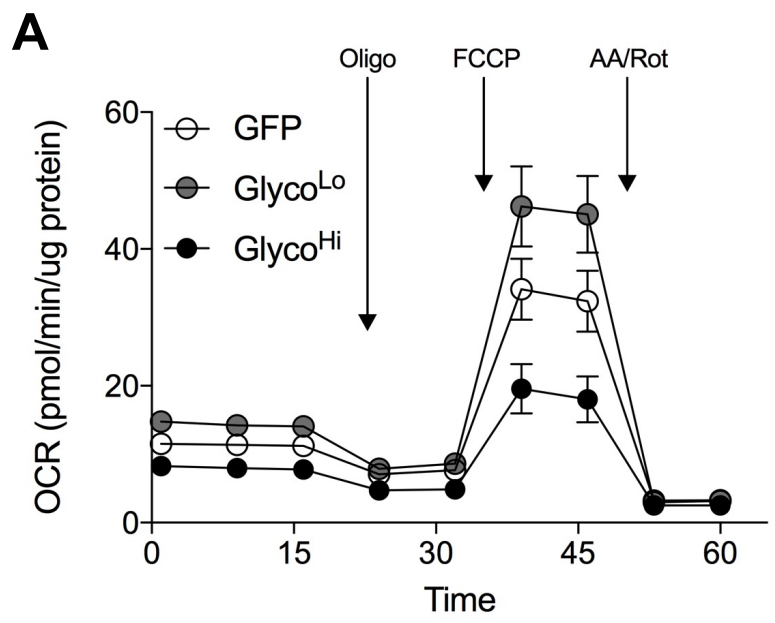

B
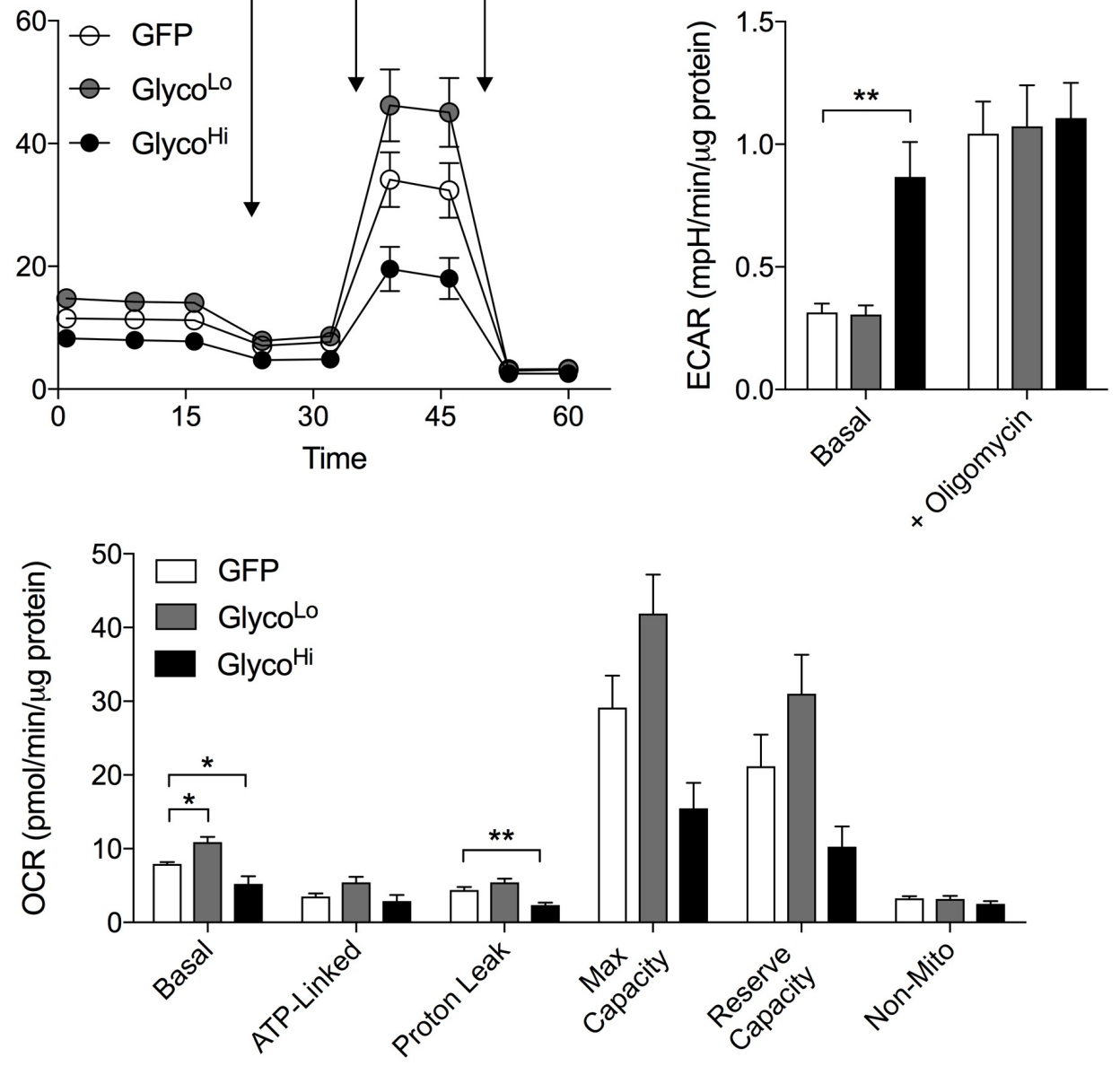
$\left.\mathrm{CO}_{2} \rightarrow \mathrm{HCO}_{3}^{-}+\mathrm{H}^{+}\right)^{290,291}$; thus, the inconsistency of ECAR with absolute measurements of glycolytic rate and ${ }^{13} \mathrm{C}_{3}$-lactate in Glyco ${ }^{\text {Lo }}$ cells (see. Fig. 1) is likely due to an increase in mitochondrial activity. Taken together, measurements from radiometric assays, stable isotope tracing, and extracellular flux analyses indicate that PFK regulates ancillary pathways by not only direct partitioning of glucose (e.g., in the PPP and the GLP) but by regulating mitochondrial cataplerotic reactions as well.

\section{$\underline{\text { Discussion }}$}

Most studies of cardiac metabolism have focused primarily on the catabolic reactions required for energy provision. ${ }^{292}$ Considerably less effort has been devoted to understanding the regulation of ancillary pathways of glucose metabolism in the heart and how these pathways integrate with catabolic reactions. In this study, we examined how PFK activity, which immediately precedes the "lysis" step in glycolysis, regulates several 6-carbon- and 3-carbon-initiated anabolic pathways that require glycolytic intermediates. Our results demonstrate that in cardiac myocytes PFK regulates glucose carbon incorporation into ancillary biosynthetic pathways by: (1) directly regulating carbon flow into purines and phospholipids; and (2) indirectly influencing mitochondrial cataplerotic activity required for the generation and subsequent incorporation of glucose-derived carbon into products of the PPP, HBP, and GLP. These studies also illustrate how radiometric measurements, stable isotope tracing, and extracellular flux analyses can be integrated to examine substrate fate and develop detailed understanding 
of how interventions simultaneously affect anabolic and catabolic metabolism.

We chose to study the integration of anabolism and catabolism in the context of glycolytic regulation at the PFK node. This enzyme controls $65 \%$ of the flux through glycolysis in the heart, ${ }^{235}$ making it the major regulator of glycolytic activity. It is unlikely that, under the experimental conditions used, glucose entry was a major limiting step for its metabolism because NRCMs express both GLUT1 and GLUT4. ${ }^{110}$ Our stable and radiometric isotope tracing measurements show that, under control conditions, $<50 \%$ of glucose taken into myocytes is catabolized past the enolase reaction. This suggests a majority of the glucose entering the myocyte is not used for energy production, but is likely used by biosynthetic pathways. Although PFK activity was found to be a potent determinant of glycolysis-engendered lactate production, it did not have diametrically coordinate effects on the relative amounts of glucose found upstream of the enolase reaction. Surprisingly, both a decrease and an increase in glycolytic activity led to the accumulation of glucose upstream of the enolase reaction; however, the mechanisms by which low and high PFK activity augment the levels of glucosederived carbon upstream of the enolase reaction appear to differ: when PFK activity was high, glucose uptake was increased, but glucose utilization was elevated; when PFK activity was low, glucose uptake was the same as that found in control cells, but glucose utilization was remarkably diminished. These results suggest that conditions of low PFK activity promote accumulation of metabolites (likely upstream of the PFK step), and that conditions of high glycolytic activity support levels of glucose-derived carbon upstream of the enolase reaction by 
augmenting glucose uptake. Additionally, our data suggest that the level of glucose available for glucose oxidation, alanine transamination, or pyruvate carboxylation was relatively stable and independent of PFK activity.

Although studies in some cell types such as adipocytes suggest that the HBP is a relatively minor pathway, utilizing only $2-3 \%$ of incoming glucose,${ }^{293}$ our stable isotope tracing data suggest that the rate of entry of glucose-derived carbon into this pathway may be higher in cardiac myocytes. The unlabeled pool of UDPHexNAc was below $10 \%$ in all groups, suggesting that sugar donors such as UDPGIcNAc and UDP-GalNAc are synthesized and utilized at high rates. In contrast, $>20 \%$ of the pool of nucleotides was unlabeled and ${ }^{13} \mathrm{C}$ enrichment of the glycerol moiety of glycerolipids was below $20 \%$. These findings appear to indicate that the HBP utilizes more glucose-derived carbon than either the PPP or the GLP. Given that up to $20 \%$ of glucose taken up by the heart could enter the PPP, ${ }^{294}$ our comparisons of ${ }^{13} \mathrm{C}$ enrichment in end products of these pathways suggests that HBP flux in cardiomyocytes may be much higher than currently appreciated.

Our results are consistent with previous findings in epithelial cells, ${ }^{144}$ fibroblasts, ${ }^{295,296}$ and developing embryos ${ }^{297}$ which show that glycolysis regulates glucose entry into the PPP. Our findings are also consistent with computational predictions of the role of PFK in regulating the PPP in the intact heart. ${ }^{235}$ Similar to findings in neurons, ${ }^{298}$ in cardiac myocytes high glycolytic rates driven by PFK decreased the incorporation of glucose carbon into the ribose ring of purines. This suggests that high rates of glycolysis decrease the flux of glucose through the oxPPP. Nevertheless, we identified remarkable differences in ${ }^{13} \mathrm{C}$ labeling in ATP 
and GTP versus CTP and UTP. Although both classes of nucleotides use the metabolic precursor ribose-5-phosphate to form the ribose ring, purine ring carbon atoms originate from $\mathrm{N}^{10}$-formyltetrahydrofolate, $\mathrm{CO}_{2}$ and glycine, while pyrimidine ring carbons come from carbamoyl phosphate and glutamine, as well as aspartate generated by cataplerosis. ${ }^{299}$ In the timeframe examined, purines did not demonstrate incorporation of carbons that derive from metabolic products of the serine biosynthetic pathway and one-carbon metabolism. Hence, it is possible that glycine and $\mathrm{N}^{10}$-formyltetrahydrofolate derived from the serine biosynthetic pathway might be inadequate to participate in purine ring synthesis during the 18 $\mathrm{h}$ incubation time used in our experiments. Alternatively, unlabeled serine and folate present in the media could be used by these cells for de novo purine biosynthesis.

The decrease in mitochondrial activity detected by extracellular flux analysis in Glyco ${ }^{\mathrm{Hi}}$ cells, paired with stable isotope tracing results in pyrimidines, suggests low cataplerotic generation of aspartate. Oxaloacetate generated in the Krebs cycle or via pyruvate carboxylation is transaminated to aspartate, which is subsequently used for pyrimidine biosynthesis. That the $m+7$ and $m+8$ isotopologues were lower in $\mathrm{Glyco}^{\mathrm{Hi}}$ cells shows that these pathways were diminished under conditions of high PFK activity. From these data, we inferred that the decrease in de novo pyrimidine biosynthesis in the $\mathrm{Glyco}^{\mathrm{Hi}}$ cells may be due to lower rates of cataplerosis. Because tricarboxylic acid anions are delicately balanced by the input and output of intermediates involved in mitochondrial substrate oxidation, anaplerosis, and cataplerosis, ${ }^{285}$ we reasoned that 
mitochondrial activity measurements could provide convergent evidence of PFKmediated regulation of auxiliary glucose biosynthetic pathways. Indeed, the decrease in mitochondrial activity in Glyco ${ }^{\mathrm{Hi}}$ cells, paired with the observation that ${ }^{13} \mathrm{C}$-aspartate entry into pyrimidines was low, suggests that inhibition of pyrimidine synthesis under conditions of high glycolytic activity is due to low rates of cataplerosis.

Similar reasoning as well as previous modeling, validation, and biochemical interpretation of ${ }^{13} \mathrm{C}$ isotopologue data for UDP-GIcNAc ${ }^{300}$ were used to determine how PFK affects the HBP, which is important given the known role of UDPhexosamines in modulating $N$ - and $O$-linked glycosylation. ${ }^{147,246}$ The fact that the unlabeled pool of UDP-HexNAc was higher in $\mathrm{Glyco}^{\mathrm{Hi}}$ cells suggests that the net effect of high PFK activity is a decrease in HBP flux, and, like that found for pyrimidines, this appears to be in part due to diminished mitochondrial activity. Nevertheless, there are limitations for interpreting current data on HBP flux. Because building UDP-HexNAc requires metabolites from many sources, it is possible that the contribution of carbon from non-labeled pools of metabolites may hinder interpretation. A potential solution to this problem would be to use a pulsechase approach, where isotopic labeling in the system is first saturated (e.g., with both ${ }^{13} \mathrm{C}$ and ${ }^{15} \mathrm{~N}$ ) and then the stably labeled substrate(s) are replaced with unlabeled substrate(s). The disappearance of the stable label over time in isotopologues of UDP-HexNAc should, with higher confidence, reveal which intersecting metabolic pathways limit HBP activity and UDP-Glc(Gal)NAc synthesis under a given set of conditions. 
To date, little evidence exists for whether or how glycolysis regulates GLP activity. Interestingly, G3P formed via glycerol-3-phosphate dehydrogenase 1 has been shown to inhibit PFK2 and activate fructose bisphosphatase $2,{ }^{301}$ suggesting feedback regulation of the GLP on both glycolytic and gluconeogenic activity. An indication that the glycolytic rate regulates GLP flux and glycerolipid synthesis is provided by studies showing that genetic deletion or overexpression of PFKFB3, a highly active form of PFK2, in adipose tissue coordinately regulates fat deposition and obesity, ${ }^{302,303}$ which is consistent with the notion that glycolysis in adipocytes regulates TAG synthesis and storage. We found that low PFK activity directly limits glucose-derived carbon incorporation into the glycerol backbone of several phospholipids including PC, PI, PE, and PS; however, this was not the case with triacylglycerols, where ${ }^{13} \mathrm{C}$ incorporation into glycerol was not different, but fatty acyl chain labeling was diminished in Glyco ${ }^{\mathrm{Hi}}$ cells. These results suggest that the fatty acid pool derived from cataplerotic reactions is differentially channeled to route newly synthesized fatty acids for esterification into triacylglycerols rather than phospholipids.

There are several limitations to our study. Although NRCMs are beating cardiac myocytes, they rely on glycolysis for energy to a much greater extent than the adult heart. ${ }^{239}$ Thus, findings regarding pathways that involve metabolites generated by cataplerosis may not entirely reflect metabolism in the adult heart. Glucose carbon fate in NRCMs appears to differ from the adult heart primarily at the level of coupling between glycolysis and glucose oxidation. In the adult human heart, previous studies show that $26 \%$ of extracted glucose undergoes oxidation 
and $12 \%$ is released as lactate. ${ }^{84}$ In contrast, in NRCMs, we show that $<13 \%$ of glucose carbon undergoes oxidation and $\sim 24 \%$ is released as lactate. Nevertheless, our finding that a large portion of exogenous glucose extracted by cardiac myocytes is apportioned for metabolic pathways other than glycolysis and glucose oxidation is supported by human in vivo isotope tracing studies in the myocardium, which demonstrate that $60 \%$ of exogenous glucose extracted by contracting myocytes enters a slow turnover pool amenable to entry into ancillary biosynthetic pathways ${ }^{84}$ Another consideration is that the NRCM culture media is devoid of fatty acids, which could influence the coupling between glycolysis and glucose oxidation as well as biosynthetic pathway activity. Last, it is important to consider that our stable isotope analyses did not include glycogen, glycine, serine, or sorbitol, which limits our understanding of how glycolysis regulates glycogen biosynthesis, serine biosynthesis, and the polyol pathway. Nevertheless, computational models created by Cortassa et al. ${ }^{235}$ suggest that the polyol pathway is under strong control by activation or inhibition of PFK. Similarly, previous studies show that PFK activity regulates glycogen abundance in the heart. $^{220,221}$ Such a behavior would be consistent with coordinate regulation of ancillary biosynthetic pathway activity by PFK.

In summary, by using several methods to measure metabolic pathway activity, we found that $>40 \%$ of glucose consumed by NRCMs is not catabolized to pyruvate, and that PFK has strong effects on the coupling between glucose oxidation and glycolysis. Pairing results from stable isotope-resolved metabolomics with extracellular flux analysis demonstrated that PFK activity 
regulates glucose carbon incorporation directly into the ribose and the glycerol moieties of purines and phospholipids, respectively, and that high PFK, by indirectly limiting mitochondrial activity, diminishes ${ }^{13} \mathrm{C}$ incorporation into pyrimidines, UDP-N-acetyl-hexosamine(s), and the fatty acyl chains of phosphatidylinositol and triglycerides. These insights could help elucidate how pathological or physiological changes in glucose metabolism regulate the anabolic behavior and remodeling of the myocardium. iii

\footnotetext{
iii This chapter previously appeared as an article in the Biochemical Journal. The original citation is as follows: Gibb AA, et al. Integration of flux measurements to resolve changes in anabolic and catabolic metabolism in cardiac myocytes. Biochem J. 2017.
} 


\section{CHAPTER V}

\section{CONCLUDING DISCUSSION}

The studies presented here were designed to develop an understanding of the effects of exercise on cardiac metabolism and the potential role of these changes in regulating the adaptive response, i.e., physiologic growth. We reasoned that, because changes in metabolism present not only a material cause but can also have direct and indirect roles in intracellular signaling, exerciseinduced changes in cardiac metabolism are essential for adaptation to training. Our general hypothesis is that exercise-induced changes in glycolytic flux are essential for activation of the physiological growth program. To test this hypothesis, we assessed the effects of acute and chronic exercise on myocardial metabolism, in particular glycolysis, and whether these changes contributed to the physiologic growth of the heart.

To establish a model in which to test our hypothesis, we first examined nocturnal/diurnal behavior, treadmill exercise compliance, and systemic as well as cardiac-specific exercise adaptations in two commonly used mouse strains, C57BL/6J, and FVB/NJ mice. As discussed in Chapter II, metabolic cage analysis indicated a strong nocturnal nature of $\mathrm{C} 57 \mathrm{BL} / 6 \mathrm{~J}$ mice, whereas $\mathrm{FVB} / \mathrm{NJ}$ mice showed no circadian element to activity, food or water intake, $\mathrm{VO}_{2}$, or $\mathrm{VCO}_{2}$. Initial 
exercise capacity tests revealed that, compared with C57BL/6J mice, FVB/NJ mice are capable of achieving nearly 2-fold higher workloads prior to exhaustion. FVB/NJ mice tested during the day were capable of achieving significantly more work compared with their night-tested counterparts. Following 4 weeks of training, FVB/NJ mice showed significant increases in exercise capacity as well as physiologic cardiac growth characterized by enlarged myocytes and higher mitochondrial DNA content. C57BL/6J mice showed no increases in exercise capacity or cardiac growth regardless of whether they exercised during the day or the night. This lack of adaptation in C57BL/6J mice was attributable, at least in part, to their progressive loss of compliance to the treadmill training protocol. From these results, we conclude that the FVB/NJ strain is a useful and robust mouse model for examining cardiac adaptations to treadmill exercise and that treadmill training during daytime hours does not negatively affect exercise compliance or capacity.

In Chapter III, we examined the effects of exercise on myocardial metabolism and the role these changes may play in contributing to exerciseinduced cardiac growth. As discussed, we found that exercise acutely decreased glucose utilization by modulating circulating substrates and reducing phosphofructokinase activity; however, upon exercise adaptation and recovery there was an increase in myocardial phosphofructokinase activity and glycolysis. To assess the relevance of these changes in glycolytic activity, we determined how cardiac-specific expression of mutant forms of 6-phosphofructo-2-kinase/fructose2,6-bisphosphatase (PFK2) affect cardiac structure, function, metabolism, and 
gene programs relevant to cardiac remodeling. Cardiac-specific expression of a kinase-deficient PFK2 transgene (Glyco ${ }^{\text {Lo }}$ mice) lowered glycolytic rate and regulated the expression of genes known to promote cardiac growth. Hearts of Glyco $^{\mathrm{LO}}$ mice had larger myocytes, enhanced cardiac function, and higher capillary-to-myocyte ratios. Expression of phosphatase-deficient PFK2 in the heart (Glyco ${ }^{\mathrm{Hi}}$ mice) increased glucose utilization and promoted a more pathological form of hypertrophy devoid of transcriptional activation of the physiologic cardiac growth program. Modulation of phosphofructokinase activity was sufficient to regulate the glucose-fatty acid cycle in the heart; however, metabolic inflexibility caused by invariantly low or high phosphofructokinase activity caused modest mitochondrial damage. Transcriptomic analyses showed that glycolysis regulates the expression of key genes involved in cardiac metabolism and remodeling. Collectively, these findings suggest that the exercise-induced decreases in glycolytic activity stimulate physiologic cardiac remodeling and that metabolic flexibility is important for maintaining mitochondrial health in the heart.

To gain insight into how these changes in glycolytic activity could contribute to cardiac growth, we assessed the impact of changes in glycolytic activity on cardiomyocyte anabolic metabolism. In Chapter IV, we show that expression of mutant forms of PFK2 in rat neonatal cardiomyocytes coordinately regulate catabolic and anabolic metabolism. An important finding was that $>40 \%$ of glucose consumed by myocytes was unaccounted for via catabolism to pyruvate, which suggests entry of glucose carbons into ancillary pathways branching from metabolites formed in the preparatory phase of glycolysis. Analysis of ${ }^{13} \mathrm{C}$ fractional 
enrichment patterns from stable isotope tracing experiments suggests that PFK activity regulates glucose carbon incorporation directly into the ribose and the glycerol moieties of purines and phospholipids, respectively. Pyrimidines, UDP-Nacetyl-hexosamine, and the fatty acyl chains of phosphatidylinositol and triglycerides showed lower ${ }^{13} \mathrm{C}$ incorporation under conditions of high PFK activity; the isotopologue ${ }^{13} \mathrm{C}$ enrichment pattern of each metabolite indicated limitations in mitochondria-engendered oxaloacetate, acetyl CoA, and fatty acids. Consistent with this notion, high glycolytic rate diminished mitochondrial activity and the coupling of glycolysis to glucose oxidation. These findings suggest that a major portion of intracellular glucose in cardiac myocytes is apportioned for ancillary biosynthetic reactions and that PFK coordinates the activities of the pentose phosphate, hexosamine biosynthetic, and glycerolipid synthesis pathways by directly modulating glycolytic intermediate entry into auxiliary glucose metabolism pathways and indirectly regulating mitochondrial cataplerosis.

Collectively, the data obtained from these studies support the notion that acute and chronic exercise is associated with changes in myocardial metabolism and that the periodic decreases in myocardial glucose utilization occurring during exercise are a stimulus for physiologic cardiac growth. Additionally, metabolic flexibility, maintained in the exercised heart, appears to be a primary distinguishing feature of mitochondrial health in the heart. These findings are summarized in Fig. 28 and are discussed in greater detail below. 
Figure 28: Exercise-induced effects on cardiac metabolism and its role in regulating cardiac growth and mitochondrial health. Acute exercise increases the circulating levels of FFA and lactate which act to decrease the utilization of glucose by the myocardium; however, in the exercise-adapted state sustained increases in circulating hormones contribute in part to enhanced glucose utilization. At the cellular level, allosteric regulation of the rate-limiting step of glycolysis, PFK1, is coordinately affected to and influence glucose utilization both during exercise and in the adapted state. These exercise-induced decreases in glycolysis are sufficient to promote physiological growth of the heart. While decreases in glucose utilization are a key trigger for cardiac growth, exerciseinduced metabolic flexibility is essential for the preservation of mitochondrial health in the physiological hypertrophied heart. 
FIGURE 28

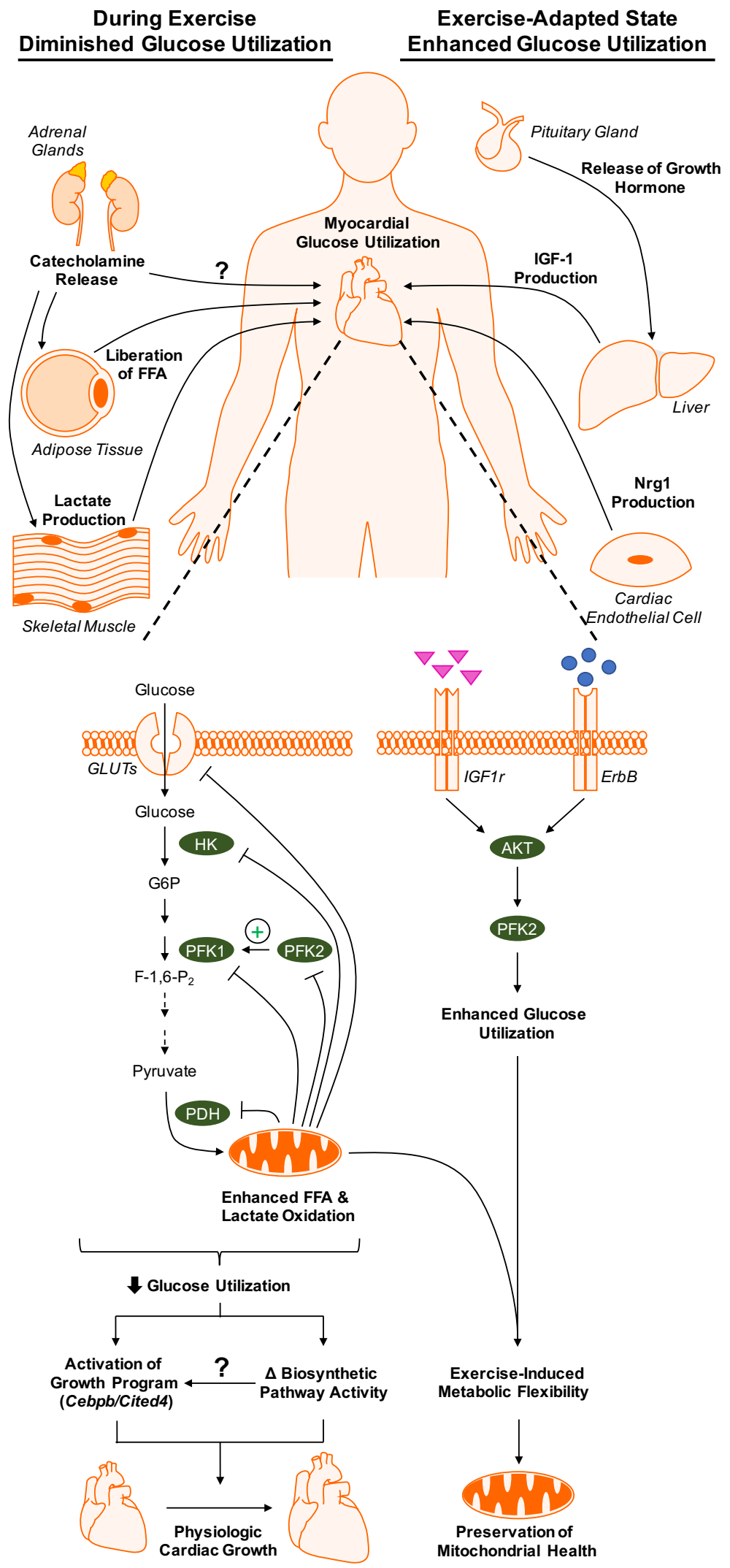




\section{Metabolic Regulation of Cardiac Growth}

While exercise promotes cardiovascular wellness and overall health, the mechanisms by which this is accomplished remain unclear. The three salient findings from our studies, related to the heart, are: (1) that periodic decreases in myocardial glycolytic activity are sufficient to promote physiologic cardiac growth $^{304}$; (2) that maintenance of metabolic flexibility in the heart is essential for mitochondrial health ${ }^{304}$; and (3) that glycolytic activity in cardiomyocytes coordinates the biosynthesis of nucleotides, nucleotide sugars, and glycero(phospho)lipids. ${ }^{305}$ Our findings have several implications, germane to both the basic understanding of cardiac remodeling as well as future targets for therapy. The finding that exercise acutely diminishes glucose utilization and that this change in metabolism activates the gene program for physiologic growth provides new knowledge and a metabolic target that could be leveraged for preventing adverse remodeling in response to stress. In particular, controlled pharmacologic modulation of the PFK node of metabolism could be used to activate the cardiac growth gene program as well as to stimulate the heart to use multiple substrates, which appears to be required for optimal mitochondrial function. Also, the finding that PFK activity regulates ancillary biosynthetic pathway activity rouses new concepts about the importance of coordinated anabolic activity in modulating hypertrophic responses to stress. These findings and their potential impact with respect to myocardial health and remodeling are discussed in detail below. 
Myocardial glycolysis coordinates cardiomyocyte biosynthetic activity: A principle finding from our studies was that PFK activity in cardiomyocytes coordinated the biosynthesis of materials required for growth (Fig. 29). One pathway affected by PFK and glycolytic activity was the PPP, which contributes to growth by: (1) replenishing NADPH levels required for anabolic reactions and the regeneration of GSH; (2) synthesizing nucleotides for DNA repair and replication; and (3) providing metabolites that can act as direct signaling modulators. Previously it has been shown that glycolysis regulates glucose entry into the PPP in several cell types. In particular, developing embryos increase PPP flux by decreasing PFK enzyme abundance, rewiring glucose metabolism for normal development. ${ }^{297}$ In the context of the adult heart, pathological hypertrophy ${ }^{132-134}$ and heart failure ${ }^{135}$ are both associated with an increase in PPP activity. During short-intensive exercise, the activities of the enzymes of the oxidative part of the PPP have been found to be slightly diminished; however, in the immediate recovery period, an increase in enzymatic activity was observed that persisted for up to $3 \mathrm{~h} .{ }^{306}$ While the effectors of increased PPP activity are not well known, a recent study identified that overexpression of PGC-1 $\alpha$ in skeletal muscle increased the enzymatic activities and end-products of the PPP. ${ }^{307}$ This finding is important as upregulation of PGC$1 \alpha$ is well-known to be initiated by acute bouts of exercise.

Our results provide additional mechanistic insight as to how PPP activity is increased in the exercising and recovering heart. Our in vivo results show that during and in the early recovery phase of exercise, PFK activity is diminished. This likely results in the redistribution of glucose into other ancillary pathways, such as 
Figure 29: Metabolic regulation of the biosynthetic pathways involved in the cardiac growth response. 


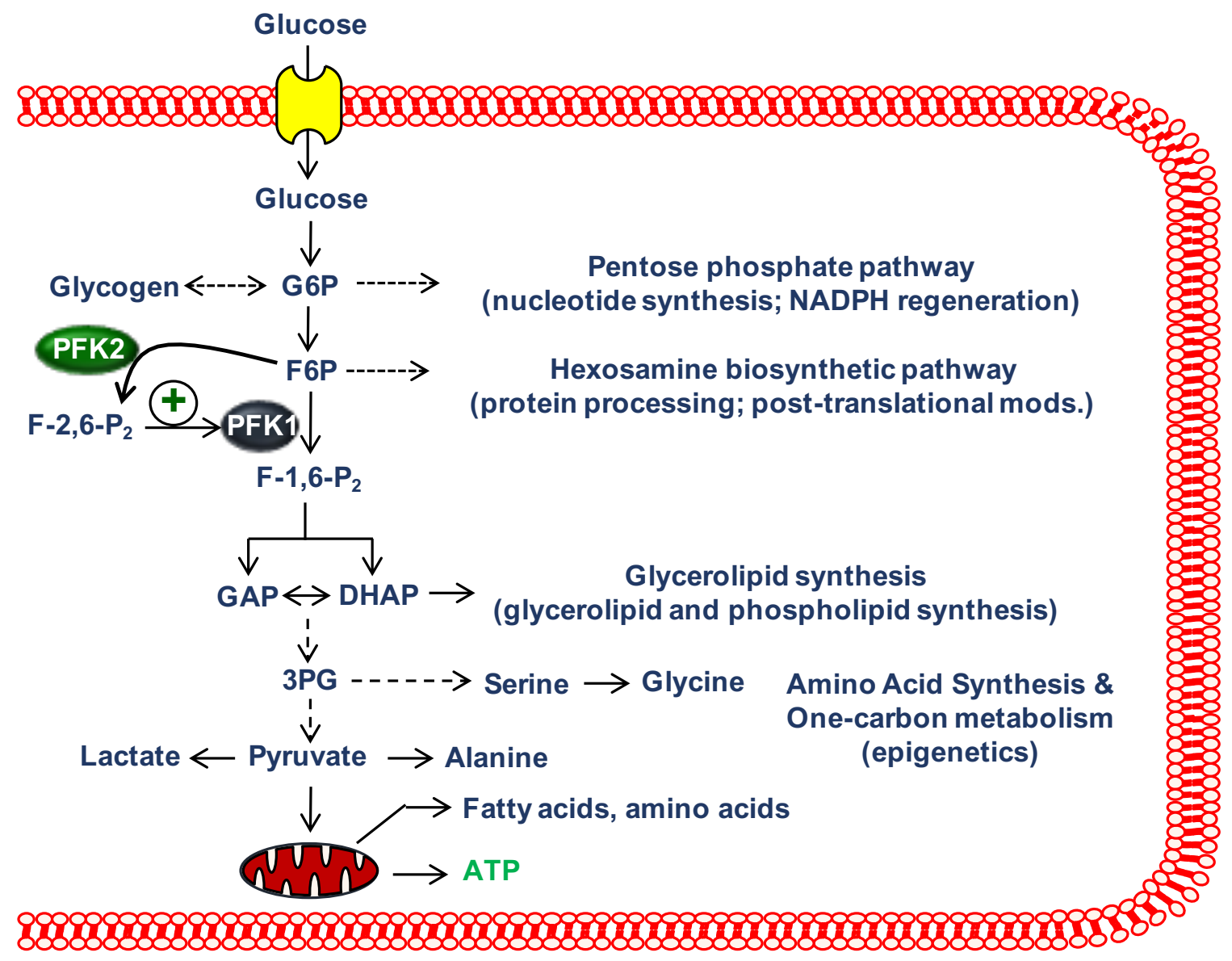


the PPP, that are upstream of PFK. In fact, several lines of evidence support this assumption, such as the increase in myocardial glycogen shown to occur at this time. Our in vivo model of diminished PFK activity (i.e., Glyco ${ }^{\text {Lo }}$ ) validated that regulation at this node of metabolism increases PPP activity as Glyco ${ }^{\text {Lo }}$ mice displayed increased abundances of several PPP-derived metabolites. Our in vitro studies also suggest that increased PPP activity may be a more coordinated and selective process, as low rates of glycolysis increased the biosynthesis of pyrimidines without affecting the purine pool.

Collectively, these results suggest that an increase in PPP activity occurs when PFK activity is diminished. This results in increased nucleotide biosynthesis for DNA replication and amino acid synthesis as well as regeneration of the NADPH pool for anabolic reactions. As growth is an anabolic event, the cell must appropriately coordinate the biosynthesis of these building blocks, priming itself for the recovery phase when growth takes place. Also, the increase in PPP activity may have a direct role in signaling the growth of the heart. Results from our metabolomic screening showed that AICAR, an AMP-analog derived from the PPP, was significantly elevated in Glyco ${ }^{\text {Lo }}$ mice compared to WT controls based on a simple unpaired t-test. This increase in AICAR was associated with a significant increase in AMPK activation (data not shown). This result could suggest that changes in PPP activity are not only important for the building blocks for growth, but also for regulating signaling pathways that instigate the growth program. 
Our in vivo and in vitro data also collectively suggest that an increase in PFK activity, such as occurs during the homeostatic phase in the exercise-adapted heart, would be important for the synthesis of glycerol(phospho)lipids. In particular, the synthesis of the glycerol moiety via the GLP is required to provide the backbone for fatty acid esterification and phospholipid synthesis. We found that the synthesis of abundant phospholipids in the cardiomyocyte, such as phosphatidylcholine, phosphotidylethanolamine, and phosphatidylinositol, ${ }^{308}$ appear to be regulated strongly by PFK-mediated changes in glycolytic activity. ${ }^{305}$ Higher levels of these phospholipids would be required for de novo expansion of cellular membranes as well as for maintaining larger myocytes, in response to stressors such as exercise.

We also found that changes in glycolytic activity cause significant phospholipid remodeling. This observation is important as it has been shown that changes in phospholipid composition, as occurs when phosphatidylethanolamine synthesis is reduced, results in impaired cardiac function. ${ }^{309}$ Similarly, chronic heart failure and diabetic cardiomyopathy, conditions in which metabolism becomes inflexible, are associated with disturbances in cardiac sarcolemmal membrane phospholipid homeostasis. ${ }^{310}$ In the exercise-adapted heart, proper phospholipid homeostasis is maintained, with little to no change in phospholipid composition. ${ }^{308}$ This could be a result of the acute periodic episodes of altered PFK and glycolytic activity instigated by exercise as opposed to the constitutive changes observed in disease. 
Metabolic flexibility and mitochondrial health: Another key finding from our studies is that metabolic flexibility appears to be essential for maintaining mitochondrial health. While it is generally accepted that mitochondrial dysfunction develops in pathologic forms of hypertrophy, where metabolism becomes inflexible, the exact mechanisms driving dysfunction remain ill-defined. Several proposed mechanisms that have been suggested to contribute to mitochondrial dysfunction include mitochondrial DNA depletion and swelling, ${ }^{311}$ disorganization and reduced density of the cristae structures, ${ }^{312}$ increased oxidative stress, ${ }^{313}$ and mitochondrial uncoupling. ${ }^{314}$ Our findings in vivo indicate that invariant inflexibility of cardiac metabolism is sufficient to promote a mild form of mitochondrial dysfunction, suggesting that the metabolic phenotypes of the heart in pathology (e.g., chronic heart failure and diabetic cardiomyopathy) may in fact initiate the decline in mitochondrial health.

In line with this idea, several of the manifestations of mitochondrial dysfunction were identified in our in vivo mouse models. In both Glyco ${ }^{\text {Lo }}$ and Glyco $^{\text {Hi }}$ hearts, we observed disorganization and a decreased density of mitochondrial cristae. Additionally, results from our metabolomics data suggest that metabolic inflexibility results in chronic oxidative stress, indicated by elevations in the lipid peroxidation product, 4-hydroxy-2-nonenal (4-HNE). Data from our in vitro studies may provide insight as to why this oxidative stress occurs, as increased rates of cardiomyocyte glycolysis diminished the biosynthesis of glutathione (data not shown), a key antioxidant. Lastly, it is possible that the mitochondria are more uncoupled which could contribute to the dysfunction 
observed. As reviewed by Lopashuk, the oxidation of FFA contributes to mitochondrial uncoupling ${ }^{82}$ Therefore, in instances of excessive FAO such as occurs in the Glyco ${ }^{\text {Lo }}$ heart, chronic uncoupling of the mitochondria could be detrimental. Our data indicate that the mitochondria from Glyco ${ }^{\text {Lo }}$ hearts are, in fact, more uncoupled than control hearts.

Mitochondrial dysfunction is also likely a consequence of changes in phospholipid homeostasis. As already discussed, both our in vivo and in vitro data indicate that changes in PFK and glycolytic activity affect phospholipid metabolism. Therefore, changes in one or several of the phospholipids found in the mitochondrial membrane could affect mitochondrial health. One of the most abundant phospholipids of the inner mitochondrial membrane is cardiolipin, which plays an important role in regulating the enzymatic activity of the electron transport chain. ${ }^{315,316}$ In heart failure, mitochondrial cardiolipin content is reduced and is correlated with mitochondrial dysfunction. ${ }^{317-319}$ While we did not measure the cardiolipin abundance or its biosynthesis, identifying whether cardiolipin metabolism is affected by changes in glycolytic activity could provide novel mechanistic insights into mitochondrial damage in disease. Taken together, these results provide evidence that metabolic flexibility is essential for the maintenance of a healthy mitochondrial pool. Furthermore, our results provide a potential therapeutic target, the PFK node of glycolysis, to increase metabolic flexibility and improve mitochondrial health in disease. 
Periodic decreases in myocardial glycolysis activate the cardiac growth program: As discussed, our data collectively indicate that changes in PFK and glycolytic activity coordinate biosynthetic pathways (e.g., PPP, GLP) that are required for cardiomyocyte growth. In addition, our data also suggest that metabolism plays a critical role in the activation of gene sets that promote cardiac growth. Most notably, our findings suggest that decreases in myocardial glycolytic rate, such as occurs during exercise, is sufficient to activate the Cebpb/Cited4 axis. While it has previously been shown that the activation of AKT was required for this transcriptional regulation, ${ }^{45}$ we found that low rates of glycolysis were sufficient to activate the physiologic gene program, suggesting that changes in metabolism are likely a proximal cause of cardiac growth.

Results from our studies suggest that PFK activity regulates anabolic pathways required for the synthesis of the amino acids alanine, serine, and glycine. The stimulation of the serine biosynthetic pathway (SBP), in particular, would not only increase serine/glycine biosynthesis, but it would control the one-carbon metabolic reactions (i.e., those involving methyl tetrahydrofolate metabolites) that are known to modulate the epigenetic landscape. ${ }^{320}$ Our data show not only that the abundance of these metabolites are changed by PFK activity (i.e., increased in $\mathrm{Glyco}^{\mathrm{Hi}}$ ), but that glycolysis regulates the transcription of enzymes involved in one-carbon metabolism (e.g., Phgdh). This could be significant because Cited4 is epigenetically regulated whereby hypermethylation of its $\mathrm{CpG}$ island causes lower expression. ${ }^{244}$ Our results are in line with the general consensus showing that altered metabolism regulates the epigenetic landscape..$^{321-323}$ While additional 
work establishing the mechanistic link between metabolism and epigenetics is required, our results provide new insights into the regulation of gene expression via metabolism.

Expanding on the observation that metabolites involved in one-carbon metabolism (e.g., serine, methionine, cysteine) are affected by PFK activity, the metabolic flexibility induced by exercise at this same metabolic node could contribute to the regulation of gene transcription. As these metabolites are involved in the methylation events of DNA and RNA, controlled modulation of the SBP pathway, by exercise-induced changes in PFK activity, could readily increase and decrease methylating agents such as 5-methyl-THF, altering gene transcription at the level of epigenetics. Our results support this notion as several genes, including Cited4, were coordinately regulated; however, we have yet to address whether changes in the SBP contribute to the physiologic gene program. The idea that PFK activity affects the epigenetic landscape is important, as it provides a potential therapeutic target that can be leveraged to prevent adverse remodeling.

\section{Future Directions}

Our results clearly demonstrate a key role for metabolism in regulating the cardiac growth response to stress; however, several questions remain unanswered. For instance, an experiment that would help to further identify how metabolic pathways integrate to promote the physiologic growth program would be to identify which pathways are similarly altered in the Glyco ${ }^{\mathrm{LO}}$ and acutely exercising heart. For example, if one-carbon metabolites involved in methylation 
are similarly reduced in their abundance, this would provide further evidence of the metabolic regulation of epigenetic modifications. Similarly, if flux through the HBP, which we show to be affected by PFK and glycolytic activity, is similarly affected in exercising and Glyco ${ }^{\text {Lo }}$ hearts, identification of the proteins that are analogously post-translationally modified could provide additional targets to further dissect the mechanisms that promote physiologic growth of the heart. Furthermore, identifying how exercise intensity and duration regulate these metabolic pathways and gene transcription would be important for optimizing cardiac adaptations and for further understanding links between metabolism and cardiac growth.

Understanding how modulation of other nodes of glucose metabolism may also provide insight as to the regulation imposed by metabolism on cardiac growth. For example, cardiac-specific pyruvate dehydrogenase complex deficiency results in a mild form of cardiac hypertrophy in mice, ${ }^{324}$ an interesting observation as it could be reasoned that this genetic modification likely results in a phenotype similar to our Glyco ${ }^{\text {Lo }}$ mouse. In mice subjected to pressure overload, cardiomyocyte overexpression of hexokinase resulted in the attenuation of pathological hypertrophy. ${ }^{139}$ Lastly, an interesting clinical finding in patients with glycogen storage diseases is the presentation of a mild form of hypertrophic cardiomyopathy. ${ }^{325}$ This suggests that myocardial glycogen contributes to the growth of the heart and may be relevant to our findings as we see that exercise and diminished PFK activity increase myocardial glycogen content. Therefore, it would be interesting to determine whether glycogen accumulation during exercise contributes to the cardiac growth response. These studies, in addition to our 
results, provide ample evidence of the role of metabolism in regulating the growth responses to stress and indicate additional nodes of glucose metabolism that potentially coordinate the biosynthetic pathways.

The conclusions from our studies also open up the possibility to explore the contributions of metabolism in other forms of cardiac growth and hypertrophy. For instance, little is known about the metabolic changes that occur in pregnancyinduced physiological hypertrophy. In healthy women, pregnancy is associated with a transient increase in cardiac size, similar to that observed in the exercised heart. ${ }^{326}$ This growth response has been linked to the activation of AKT and ERK1/2 signaling pathways, both known to influence and control cellular metabolism. ${ }^{327-329}$ with this knowledge in hand, it would be interesting to test whether pregnancy-induced cardiac growth is associated with similar changes in PFK activity and transcription regulation of Cebpb and Cited4 as we observed in our exercise model.

The transcriptomics results from our study provided a large data set in which to ask new and exciting questions in the field of cardiac health in disease. For example, the expression of gluconeogenic genes, fructose-1,6-bisphosphatase 2 (Fbp2) and phosphoenolpyruvate (Pck1), were found to be altered by glycolytic activity in the heart, with Fbp2 in a coordinate manner. The fact that these genes are modifiable in an organ thought to have limited gluconeogenic potential is noteworthy. This observation provides the opportunity to test the hypothesis that an increase in myocardial gluconeogenic enzyme activity would be cardioprotective from pathologic insult or promote myocyte proliferation by 
increasing the bioavailability of carbons for ancillary biosynthetic flux and through regulation of gene transcription. Similarly, we found that centromere protein $F$ (Cenpf), a gene associated with chromosome segregation during mitosis, ${ }^{330}$ was found to be coordinately regulated by glycolysis. Interrogation of the metabolic regulation of such cell division genes in cardiomyocytes, thought to be terminally differentiated and relatively incapable of division and proliferation, may provide novel therapeutic approaches to regenerate the myocardium following injury.

In conclusion, we demonstrate a clear role of exercise-induced changes in metabolism in coordinating the growth response of the heart and the importance of metabolic flexibility in maintaining cardiac health. It is my hope that the knowledge derived from these studies will form the basis for the development of novel interventions to improve cardiac health (e.g., in patients with heart failure or diabetic cardiomyopathy). 


\section{REFERENCES}

1. Hippocrates, Heraclitus, Jones WHS, Potter P, Smith WD and Withington ET. Hippocrates. Loeb Classical Library 147-150, 472-473, 477, 482, 509, 520. 2014:1 online resource line illustrations, maps.

2. Booth FW and Lees SJ. Physically active subjects should be the control group. Med Sci Sports Exerc. 2006;38:405-6.

3. WHO. Global health risks: mortality and burden of disease attributable to selected major risks (Geneva, Switzerland: World Health Organization). 2009.

4. Morris JN, Heady JA, Raffle PA, Roberts CG and Parks JW. Coronary heart-disease and physical activity of work. Lancet. 1953;265:1111-20; concl.

5. Blair SN, Kampert JB, Kohl HW, 3rd, Barlow CE, Macera CA, Paffenbarger RS, Jr. and Gibbons LW. Influences of cardiorespiratory fitness and other precursors on cardiovascular disease and all-cause mortality in men and women. Jama. 1996;276:205-10.

6. Joyner MJ and Green DJ. Exercise protects the cardiovascular system: effects beyond traditional risk factors. The Journal of physiology. 2009;587:5551-8. 
7. Mora S, Cook N, Buring JE, Ridker PM and Lee IM. Physical activity and reduced risk of cardiovascular events: potential mediating mechanisms. Circulation. 2007;116:2110-8.

8. Egan B and Zierath JR. Exercise metabolism and the molecular regulation of skeletal muscle adaptation. Cell metabolism. 2013;17:162-84.

9. de Cabo R, Carmona-Gutierrez D, Bernier M, Hall MN and Madeo F. The search for antiaging interventions: from elixirs to fasting regimens. Cell. 2014;157:1515-26.

10. Mercken EM, Carboneau BA, Krzysik-Walker SM and de Cabo R. Of mice and men: the benefits of caloric restriction, exercise, and mimetics. Ageing research reviews. 2012;11:390-8.

11. Blair SN, Kohl HW, 3rd, Paffenbarger RS, Jr., Clark DG, Cooper KH and Gibbons LW. Physical fitness and all-cause mortality. A prospective study of healthy men and women. Jama. 1989;262:2395-401.

12. Myers J, Prakash M, Froelicher V, Do D, Partington S and Atwood JE. Exercise capacity and mortality among men referred for exercise testing. The New England journal of medicine. 2002;346:793-801.

13. Paffenbarger RS, Jr., Hyde RT, Wing AL and Hsieh CC. Physical activity, all-cause mortality, and longevity of college alumni. The New England journal of medicine. 1986;314:605-13.

14. Brooks AB, Fahey, T. D., Baldwin, K. M. Exercise Physiology: Human Bioenergetics and Its Applications 4th Edition. 2005. 
15. MacDougall JD, Tuxen D, Sale DG, Moroz JR and Sutton JR. Arterial blood pressure response to heavy resistance exercise. J Appl Physiol (1985). 1985;58:785-90.

16. Andrews Portes L, Magalhaes Saraiva R, Alberta Dos Santos A and Tucci PJ. Swimming training attenuates remodeling, contractile dysfunction and congestive heart failure in rats with moderate and large myocardial infarctions. Clin Exp Pharmacol Physiol. 2009;36:394-9.

17. Barboza CA, Rocha LY, Mostarda CT, Figueroa D, Caperuto EC, De Angelis $\mathrm{K}$, Irigoyen $\mathrm{MC}$ and Rodrigues $\mathrm{B}$. Ventricular and autonomic benefits of exercise training persist after detraining in infarcted rats. Eur $J$ Appl Physiol. 2013;113:1137-46.

18. Xu T, Tang H, Zhang B, Cai C, Liu X, Han Q and Zou L. Exercise preconditioning attenuates pressure overload-induced pathological cardiac hypertrophy. Int J Clin Exp Pathol. 2015;8:530-40.

19. Stolen TO, Hoydal MA, Kemi OJ, Catalucci D, Ceci M, Aasum E, Larsen T, Rolim N, Condorelli G, Smith GL and Wisloff U. Interval training normalizes cardiomyocyte function, diastolic $\mathrm{Ca} 2+$ control, and $\mathrm{SR} \mathrm{Ca} 2+$ release synchronicity in a mouse model of diabetic cardiomyopathy. Circ Res. 2009;105:527-36.

20. Pluim BM, Zwinderman AH, van der Laarse $A$ and van der Wall EE. The athlete's heart. A meta-analysis of cardiac structure and function. Circulation. 2000;101:336-44. 
21. Convertino VA. Blood volume: its adaptation to endurance training. Med Sci Sports Exerc. 1991;23:1338-48.

22. Kelley GA, Kelley KA and Tran ZV. Aerobic exercise and resting blood pressure: a meta-analytic review of randomized, controlled trials. Prev Cardiol. 2001;4:73-80.

23. Kemi OJ and Wisloff U. Mechanisms of exercise-induced improvements in the contractile apparatus of the mammalian myocardium. Acta Physiol (Oxf). 2010;199:425-39.

24. Wisloff U, Loennechen JP, Falck G, Beisvag V, Currie S, Smith G and Ellingsen $O$. Increased contractility and calcium sensitivity in cardiac myocytes isolated from endurance trained rats. Cardiovasc Res. 2001;50:495-508.

25. Kemi OJ, Ellingsen O, Ceci M, Grimaldi S, Smith GL, Condorelli G and Wisloff U. Aerobic interval training enhances cardiomyocyte contractility and Ca2+ cycling by phosphorylation of CaMKII and Thr-17 of phospholamban. J Mol Cell Cardiol. 2007;43:354-61.

26. Bernardo BC, Weeks KL, Pretorius $L$ and McMullen JR. Molecular distinction between physiological and pathological cardiac hypertrophy: experimental findings and therapeutic strategies. Pharmacol Ther. 2010;128:191-227.

27. Maillet M, van Berlo JH and Molkentin JD. Molecular basis of physiological heart growth: fundamental concepts and new players. Nature reviews Molecular cell biology. 2013;14:38-48. 
28. Ehsani AA, Hagberg JM and Hickson RC. Rapid changes in left ventricular dimensions and mass in response to physical conditioning and deconditioning. Am J Cardiol. 1978;42:52-6.

29. Maron BJ, Pelliccia A, Spataro A and Granata M. Reduction in left ventricular wall thickness after deconditioning in highly trained Olympic athletes. Br Heart J. 1993;69:125-8.

30. Wilkins BJ, Dai YS, Bueno OF, Parsons SA, Xu J, Plank DM, Jones F, Kimball TR and Molkentin JD. Calcineurin/NFAT coupling participates in pathological, but not physiological, cardiac hypertrophy. Circ Res. 2004;94:110-8.

31. Sutton $\mathrm{J}$ and Lazarus L. Growth hormone in exercise: comparison of physiological and pharmacological stimuli. J Appl Physiol. 1976;41:523-7.

32. Neri Serneri GG, Boddi M, Modesti PA, Cecioni I, Coppo M, Padeletti L, Michelucci A, Colella A and Galanti G. Increased cardiac sympathetic activity and insulin-like growth factor-I formation are associated with physiological hypertrophy in athletes. Circ Res. 2001;89:977-82.

33. Liu JP, Baker J, Perkins AS, Robertson EJ and Efstratiadis A. Mice carrying null mutations of the genes encoding insulin-like growth factor I (Igf-1) and type 1 IGF receptor (Igf1r). Cell. 1993;75:59-72.

34. Delaughter MC, Taffet GE, Fiorotto ML, Entman ML and Schwartz RJ. Local insulin-like growth factor I expression induces physiologic, then pathologic, cardiac hypertrophy in transgenic mice. FASEB J. 1999;13:1923-9. 
35. Kim J, Wende AR, Sena S, Theobald HA, Soto J, Sloan C, Wayment BE, Litwin SE, Holzenberger M, LeRoith D and Abel ED. Insulin-like growth factor I receptor signaling is required for exercise-induced cardiac hypertrophy. Mol Endocrinol. 2008;22:2531-43.

36. McMullen JR, Shioi T, Huang WY, Zhang L, Tarnavski O, Bisping E, Schinke M, Kong S, Sherwood MC, Brown J, Riggi L, Kang PM and Izumo $\mathrm{S}$. The insulin-like growth factor 1 receptor induces physiological heart growth via the phosphoinositide 3-kinase(p110alpha) pathway. J Biol Chem. 2004;279:4782-93.

37. Shioi T, Kang PM, Douglas PS, Hampe J, Yballe CM, Lawitts J, Cantley LC and Izumo S. The conserved phosphoinositide 3-kinase pathway determines heart size in mice. EMBO J. 2000;19:2537-48.

38. Crackower MA, Oudit GY, Kozieradzki I, Sarao R, Sun H, Sasaki T, Hirsch E, Suzuki A, Shioi T, Irie-Sasaki J, Sah R, Cheng HY, Rybin VO, Lembo G, Fratta L, Oliveira-dos-Santos AJ, Benovic JL, Kahn CR, Izumo S, Steinberg SF, Wymann MP, Backx PH and Penninger JM. Regulation of myocardial contractility and cell size by distinct PI3K-PTEN signaling pathways. Cell. 2002;110:737-49.

39. Noh J, Wende AR, Olsen CD, Kim B, Bevins J, Zhu Y, Zhang QJ, Riehle C and Abel ED. Phosphoinositide dependent protein kinase 1 is required for exercise-induced cardiac hypertrophy but not the associated mitochondrial adaptations. J Mol Cell Cardiol. 2015;89:297-305. 
40. Riehle C, Wende AR, Zhu Y, Oliveira KJ, Pereira RO, Jaishy BP, Bevins J, Valdez S, Noh J, Kim BJ, Moreira AB, Weatherford ET, Manivel R, Rawlings TA, Rech M, White MF and Abel ED. Insulin receptor substrates are essential for the bioenergetic and hypertrophic response of the heart to exercise training. Mol Cell Biol. 2014;34:3450-60.

41. Shiojima I, Yefremashvili M, Luo Z, Kureishi Y, Takahashi A, Tao J, Rosenzweig A, Kahn CR, Abel ED and Walsh K. Akt signaling mediates postnatal heart growth in response to insulin and nutritional status. $J$ Biol Chem. 2002;277:37670-7.

42. Wende AR, O'Neill BT, Bugger H, Riehle C, Tuinei J, Buchanan J, Tsushima K, Wang L, Caro P, Guo A, Sloan C, Kim BJ, Wang X, Pereira RO, McCrory MA, Nye BG, Benavides GA, Darley-Usmar VM, Shioi T, Weimer BC and Abel ED. Enhanced cardiac Akt/protein kinase B signaling contributes to pathological cardiac hypertrophy in part by impairing mitochondrial function via transcriptional repression of mitochondrion-targeted nuclear genes. $\mathrm{Mol}$ Cell Biol. 2015;35:831-46.

43. DeBosch B, Treskov I, Lupu TS, Weinheimer C, Kovacs A, Courtois M and Muslin AJ. Akt1 is required for physiological cardiac growth. Circulation. 2006;113:2097-104.

44. Shioi T, McMullen JR, Kang PM, Douglas PS, Obata T, Franke TF, Cantley LC and Izumo S. Akt/protein kinase B promotes organ growth in transgenic mice. Mol Cell Biol. 2002;22:2799-809. 
45. Bostrom P, Mann N, Wu J, Quintero PA, Plovie ER, Panakova D, Gupta RK, Xiao C, MacRae CA, Rosenzweig A and Spiegelman BM. C/EBPbeta controls exercise-induced cardiac growth and protects against pathological cardiac remodeling. Cell. 2010;143:1072-83.

46. Bezzerides VJ, Platt C, Lerchenmuller C, Paruchuri K, Oh NL, Xiao C, Cao Y, Mann N, Spiegelman BM and Rosenzweig A. CITED4 induces physiologic hypertrophy and promotes functional recovery after ischemic injury. JCI Insight. 2016;1.

47. Odiete O, Hill MF and Sawyer DB. Neuregulin in cardiovascular development and disease. Circ Res. 2012;111:1376-85.

48. Bueno OF, De Windt LJ, Tymitz KM, Witt SA, Kimball TR, Klevitsky R, Hewett TE, Jones SP, Lefer DJ, Peng CF, Kitsis RN and Molkentin JD. The MEK1-ERK1/2 signaling pathway promotes compensated cardiac hypertrophy in transgenic mice. EMBO J. 2000;19:6341-50.

49. Ali SR, Hippenmeyer S, Saadat LV, Luo L, Weissman IL and Ardehali R. Existing cardiomyocytes generate cardiomyocytes at a low rate after birth in mice. Proc Natl Acad Sci U S A. 2014;111:8850-5.

50. Bergmann O, Bhardwaj RD, Bernard S, Zdunek S, Barnabe-Heider F, Walsh S, Zupicich J, Alkass K, Buchholz BA, Druid H, Jovinge S and Frisen J. Evidence for cardiomyocyte renewal in humans. Science. 2009;324:98102. 
51. Senyo SE, Steinhauser ML, Pizzimenti CL, Yang VK, Cai L, Wang M, Wu TD, Guerquin-Kern JL, Lechene CP and Lee RT. Mammalian heart renewal by pre-existing cardiomyocytes. Nature. 2013;493:433-6.

52. Platt $\mathrm{C}$, Houstis $\mathrm{N}$ and Rosenzweig $\mathrm{A}$. Using exercise to measure and modify cardiac function. Cell metabolism. 2015;21:227-36.

53. Waring CD, Vicinanza C, Papalamprou A, Smith AJ, Purushothaman S, Goldspink DF, Nadal-Ginard B, Torella D and Ellison GM. The adult heart responds to increased workload with physiologic hypertrophy, cardiac stem cell activation, and new myocyte formation. Eur Heart J. 2014;35:2722-31.

54. Xiao J, Xu T, Li J, Lv D, Chen P, Zhou Q and Xu J. Exercise-induced physiological hypertrophy initiates activation of cardiac progenitor cells. Int J Clin Exp Pathol. 2014;7:663-9.

55. Calvert JW, Condit ME, Aragon JP, Nicholson CK, Moody BF, Hood RL, Sindler AL, Gundewar S, Seals DR, Barouch LA and Lefer DJ. Exercise protects against myocardial ischemia-reperfusion injury via stimulation of beta(3)-adrenergic receptors and increased nitric oxide signaling: role of nitrite and nitrosothiols. Circ Res. 2011;108:1448-58.

56. Dimmeler S, Fleming I, Fisslthaler B, Hermann C, Busse R and Zeiher AM. Activation of nitric oxide synthase in endothelial cells by Akt-dependent phosphorylation. Nature. 1999;399:601-5.

57. Zhang QJ, McMillin SL, Tanner JM, Palionyte M, Abel ED and Symons JD. Endothelial nitric oxide synthase phosphorylation in treadmill-running mice: 
role of vascular signalling kinases. The Journal of physiology. 2009;587:3911-20.

58. White FC, Bloor CM, McKirnan MD and Carroll SM. Exercise training in swine promotes growth of arteriolar bed and capillary angiogenesis in heart. J Appl Physiol (1985). 1998;85:1160-8.

59. Kraus RM, Stallings HW, 3rd, Yeager RC and Gavin TP. Circulating plasma VEGF response to exercise in sedentary and endurance-trained men. $J$ Appl Physiol (1985). 2004;96:1445-50.

60. Rehman J, Li J, Parvathaneni L, Karlsson G, Panchal VR, Temm CJ, Mahenthiran $\mathrm{J}$ and March KL. Exercise acutely increases circulating endothelial progenitor cells and monocyte-/macrophage-derived angiogenic cells. J Am Coll Cardiol. 2004;43:2314-8.

61. Rimbaud S, Garnier A and Ventura-Clapier R. Mitochondrial biogenesis in cardiac pathophysiology. Pharmacol Rep. 2009;61:131-8.

62. White FC, McKirnan MD, Breisch EA, Guth BD, Liu YM and Bloor CM. Adaptation of the left ventricle to exercise-induced hypertrophy. J Appl Physiol (1985). 1987;62:1097-110.

63. Gibb AA, McNally LA, Riggs DW, Conklin DJ, Bhatnagar A and Hill BG. FVB/NJ Mice Are a Useful Model for Examining Cardiac Adaptations to Treadmill Exercise Frontiers in Physiology 2016;7:636.

64. Perry CG, Lally J, Holloway GP, Heigenhauser GJ, Bonen A and Spriet LL. Repeated transient mRNA bursts precede increases in transcriptional and 
mitochondrial proteins during training in human skeletal muscle. The Journal of physiology. 2010;588:4795-810.

65. O'Neill BT, Kim J, Wende AR, Theobald HA, Tuinei J, Buchanan J, Guo A, Zaha VG, Davis DK, Schell JC, Boudina S, Wayment B, Litwin SE, Shioi T, Izumo S, Birnbaum MJ and Abel ED. A conserved role for phosphatidylinositol 3-kinase but not Akt signaling in mitochondrial adaptations that accompany physiological cardiac hypertrophy. Cell metabolism. 2007;6:294-306.

66. Burelle Y, Wambolt RB, Grist M, Parsons HL, Chow JC, Antler C, Bonen A, Keller A, Dunaway GA, Popov KM, Hochachka PW and Allard MF. Regular exercise is associated with a protective metabolic phenotype in the rat heart. Am J Physiol Heart Circ Physiol. 2004;287:H1055-63.

67. Strom CC, Aplin M, Ploug T, Christoffersen TE, Langfort J, Viese M, Galbo $\mathrm{H}$, Haunso S and Sheikh SP. Expression profiling reveals differences in metabolic gene expression between exercise-induced cardiac effects and maladaptive cardiac hypertrophy. FEBS J. 2005;272:2684-95.

68. Neely JR and Morgan HE. Relationship between carbohydrate and lipid metabolism and the energy balance of heart muscle. Annu Rev Physiol. 1974;36:413-59.

69. Opie LH. Metabolism of the heart in health and disease. I. Am Heart J. 1968;76:685-98.

70. Opie LH. Metabolism of the heart in health and disease. II. Am Heart J. 1969;77:100-22 contd. 
71. Opie LH. Heart Physiology: From Cell to Circulation. Philadelphia, PA: Lippincott Williams \& Wilkins. 2004.

72. Gibbs CL. Cardiac energetics. Physiol Rev. 1978;58:174-254.

73. Suga H. Ventricular energetics. Physiol Rev. 1990;70:247-77.

74. Lassers BW, Kaijser L and Carlson LA. Myocardial lipid and carbohydrate metabolism in healthy, fasting men at rest: studies during continuous infusion of 3 H-palmitate. Eur J Clin Invest. 1972;2:348-58.

75. Lassers BW, Kaijser L, Wahlqvist ML and Carlson LA. Relationship in man between plasma free fatty acids and myocardial metabolism of carbohydrate substrates. Lancet. 1971;2:448-50.

76. Nuutila P, Koivisto VA, Knuuti J, Ruotsalainen U, Teras M, Haaparanta M, Bergman J, Solin O, Voipio-Pulkki LM, Wegelius U and et al. Glucose-free fatty acid cycle operates in human heart and skeletal muscle in vivo. J Clin Invest. 1992;89:1767-74.

77. Wisneski JA, Gertz EW, Neese RA, Gruenke LD, Morris DL and Craig JC. Metabolic fate of extracted glucose in normal human myocardium. J Clin Invest. 1985;76:1819-27.

78. Goodwin GW and Taegtmeyer H. Improved energy homeostasis of the heart in the metabolic state of exercise. Am J Physiol Heart Circ Physiol. 2000;279:H1490-501.

79. Schonekess BO. Competition between lactate and fatty acids as sources of ATP in the isolated working rat heart. J Mol Cell Cardiol. 1997;29:2725-33. 
80. Randle PJ, Garland PB, Hales CN and Newsholme EA. The glucose fattyacid cycle. Its role in insulin sensitivity and the metabolic disturbances of diabetes mellitus. Lancet. 1963;1:785-9.

81. Gibala MJ, MacLean DA, Graham TE and Saltin B. Tricarboxylic acid cycle intermediate pool size and estimated cycle flux in human muscle during exercise. Am J Physiol. 1998;275:E235-42.

82. Lopaschuk GD, Ussher JR, Folmes CD, Jaswal JS and Stanley WC. Myocardial fatty acid metabolism in health and disease. Physiol Rev. 2010;90:207-58.

83. Goodwin GW, Taylor CS and Taegtmeyer H. Regulation of energy metabolism of the heart during acute increase in heart work. J Biol Chem. 1998;273:29530-9.

84. Gertz EW, Wisneski JA, Stanley WC and Neese RA. Myocardial substrate utilization during exercise in humans. Dual carbon-labeled carbohydrate isotope experiments. J Clin Invest. 1988;82:2017-25.

85. Kaijser $L$ and Berglund B. Myocardial lactate extraction and release at rest and during heavy exercise in healthy men. Acta Physiol Scand. 1992;144:39-45.

86. Kemppainen J, Fujimoto T, Kalliokoski KK, Viljanen T, Nuutila P and Knuuti J. Myocardial and skeletal muscle glucose uptake during exercise in humans. The Journal of physiology. 2002;542:403-12.

87. Lassers BW, Kaijser L, Wahlqvist M and Carlson LA. Effect of prolonged exercise on myocardial metabolism in man. Br Heart J. 1971;33:609. 
88. Stanley WC, Recchia FA and Lopaschuk GD. Myocardial substrate metabolism in the normal and failing heart. Physiol Rev. 2005;85:1093-129.

89. Rodahl K, Miller HI and Issekutz B, Jr. Plasma Free Fatty Acids in Exercise. J Appl Physiol. 1964;19:489-92.

90. Lassers BW, Wahlqvist ML, Kaijser $L$ and Carlson LA. Effect of nicotinic acid on myocardial metabolism in man at rest and during exercise. $J$ Appl Physiol. 1972;33:72-80.

91. Kraemer FB and Shen WJ. Hormone-sensitive lipase: control of intracellular tri-(di-)acylglycerol and cholesteryl ester hydrolysis. J Lipid Res. 2002;43:1585-94.

92. Bonen A, Luiken JJ, Arumugam Y, Glatz JF and Tandon NN. Acute regulation of fatty acid uptake involves the cellular redistribution of fatty acid translocase. J Biol Chem. 2000;275:14501-8.

93. Jeppesen J, Albers P, Luiken JJ, Glatz JF and Kiens B. Contractions but not AICAR increase FABPpm content in rat muscle sarcolemma. Mol Cell Biochem. 2009;326:45-53.

94. Wisneski JA, Gertz EW, Neese RA and Mayr M. Myocardial metabolism of free fatty acids. Studies with 14 C-labeled substrates in humans. J Clin Invest. 1987;79:359-66.

95. Saddik M and Lopaschuk GD. Myocardial triglyceride turnover and contribution to energy substrate utilization in isolated working rat hearts. $J$ Biol Chem. 1991;266:8162-70. 
96. de Groot MJ, Coumans WA, Willemsen PH and van der Vusse GJ. Substrate-induced changes in the lipid content of ischemic and reperfused myocardium. Its relation to hemodynamic recovery. Circ Res. 1993;72:17686.

97. Gertz EW, Wisneski JA, Neese R, Bristow JD, Searle GL and Hanlon JT. Myocardial lactate metabolism: evidence of lactate release during net chemical extraction in man. Circulation. 1981;63:1273-9.

98. Wisneski JA, Gertz EW, Neese RA, Gruenke LD and Craig JC. Dual carbonlabeled isotope experiments using D-[6-14C] glucose and L-[1,2,3-13C3] lactate: a new approach for investigating human myocardial metabolism during ischemia. J Am Coll Cardiol. 1985;5:1138-46.

99. Bertrand ME, Carre AG, Ginestet AP, Lefebvre JM, Desplanque LA and Lekieffre JP. Maximal exercise in normal subjects: changes in coronary sinus blood flow, contractility and myocardial extraction of FFA and lactate. Eur J Cardiol. 1977;5:481-91.

100. Drake AJ, Haines JR and Noble MI. Preferential uptake of lactate by the normal myocardium in dogs. Cardiovasc Res. 1980;14:65-72.

101. Keul J. Myocardial metabolism in athletes. Adv Exp Med Biol. 1971;11:447467.

102. Stanley WC, Gertz EW, Wisneski JA, Morris DL, Neese RA and Brooks GA. Systemic lactate kinetics during graded exercise in man. Am J Physiol. 1985;249:E595-602. 
103. Kainulainen $\mathrm{H}$, Komulainen J, Leinonen A, Rusko $\mathrm{H}$ and Vihko V. Regional differences of substrate oxidation capacity in rat hearts: effects of extra load and endurance training. Basic Res Cardiol. 1990;85:630-9.

104. Ji LL, Stratman FW and Lardy HA. Chronic exercise training alters kinetic properties of rat skeletal muscle and myocardial lactate dehydrogenase. FEBS Lett. 1986;208:297-300.

105. Baker SK, McCullagh KJ and Bonen A. Training intensity-dependent and tissue-specific increases in lactate uptake and MCT-1 in heart and muscle. J Appl Physiol (1985). 1998;84:987-94.

106. Levy B, Mansart A, Montemont C, Gibot S, Mallie JP, Regnault V, Lecompte $\mathrm{T}$ and Lacolley P. Myocardial lactate deprivation is associated with decreased cardiovascular performance, decreased myocardial energetics, and early death in endotoxic shock. Intensive Care Med. 2007;33:495-502.

107. Bing RJ. The metabolism of the heart. Trans Am Coll Cardiol. 1955;5:8-14.

108. Hinkle PC. P/O ratios of mitochondrial oxidative phosphorylation. Biochim Biophys Acta. 2005;1706:1-11.

109. Takala TE, Ruskoaho HJ and Hassinen IE. Transmural distribution of cardiac glucose uptake in rat during physical exercise. Am J Physiol. 1983;244:H131-7.

110. Shao D and Tian R. Glucose Transporters in Cardiac Metabolism and Hypertrophy. Compr Physiol. 2015;6:331-51. 
111. Doenst $\mathrm{T}$ and Taegtmeyer $\mathrm{H}$. alpha-adrenergic stimulation mediates glucose uptake through phosphatidylinositol 3-kinase in rat heart. Circ Res. 1999;84:467-74.

112. Rattigan S, Appleby GJ and Clark MG. Insulin-like action of catecholamines and $\mathrm{Ca} 2+$ to stimulate glucose transport and GLUT4 translocation in perfused rat heart. Biochim Biophys Acta. 1991;1094:217-23.

113. Coven DL, Hu X, Cong L, Bergeron R, Shulman GI, Hardie DG and Young LH. Physiological role of AMP-activated protein kinase in the heart: graded activation during exercise. American journal of physiology Endocrinology and metabolism. 2003;285:E629-36.

114. Redmon JB, Kubo SH and Robertson RP. Glucose, insulin, and glucagon levels during exercise in pancreas transplant recipients. Diabetes Care. 1995;18:457-62.

115. Watt MJ, Heigenhauser GJ, LeBlanc PJ, Inglis JG, Spriet LL and Peters SJ. Rapid upregulation of pyruvate dehydrogenase kinase activity in human skeletal muscle during prolonged exercise. J Appl Physiol (1985). 2004;97:1261-7.

116. Doenst T, Nguyen TD and Abel ED. Cardiac metabolism in heart failure: implications beyond ATP production. Circ Res. 2013;113:709-24.

117. Goodwin GW, Arteaga JR and Taegtmeyer H. Glycogen turnover in the isolated working rat heart. J Biol Chem. 1995;270:9234-40.

118. Goodwin GW, Ahmad F and Taegtmeyer H. Preferential oxidation of glycogen in isolated working rat heart. J Clin Invest. 1996;97:1409-16. 
119. Henning SL, Wambolt RB, Schonekess BO, Lopaschuk GD and Allard MF. Contribution of glycogen to aerobic myocardial glucose utilization. Circulation. 1996;93:1549-55.

120. Evans G. The glycogen content of the rat heart. The Journal of physiology. 1934;82:468-80.

121. Reichelt ME, Mellor KM, Curl CL, Stapleton D and Delbridge LM. Myocardial glycophagy - a specific glycogen handling response to metabolic stress is accentuated in the female heart. J Mol Cell Cardiol. 2013;65:67-75.

122. Schneider $\mathrm{CA}$ and Taegtmeyer $\mathrm{H}$. Fasting in vivo delays myocardial cell damage after brief periods of ischemia in the isolated working rat heart. Circ Res. 1991;68:1045-50.

123. Depre C, Veitch K and Hue L. Role of fructose 2,6-bisphosphate in the control of glycolysis. Stimulation of glycogen synthesis by lactate in the isolated working rat heart. Acta Cardiol. 1993;48:147-64.

124. Laughlin MR, Taylor J, Chesnick AS and Balaban RS. Nonglucose substrates increase glycogen synthesis in vivo in dog heart. Am J Physiol. 1994;267:H219-23.

125. Rudolph W, Maas D, Richter J, Hasinger F, Hofmann H and Dohrn P. [on the Significance of Acetoacetate and Beta-Hydroxybutyrate in Human Myocardial Metabolism]. Klin Wochenschr. 1965;43:445-51.

126. Zimmerman AN, Meijler FL and Hulsmann WC. The inhibitory effect of acetoacetate on myocardial contraction. Lancet. 1962;2:757-8. 
127. Taegtmeyer $\mathrm{H}$, Hems $\mathrm{R}$ and Krebs HA. Utilization of energy-providing substrates in the isolated working rat heart. Biochem J. 1980;186:701-11.

128. Aubert G, Martin OJ, Horton JL, Lai L, Vega RB, Leone TC, Koves T, Gardell SJ, Kruger M, Hoppel CL, Lewandowski ED, Crawford PA, Muoio DM and Kelly DP. The Failing Heart Relies on Ketone Bodies as a Fuel. Circulation. 2016;133:698-705.

129. Ichihara K, Neely JR, Siehl DL and Morgan HE. Utilization of leucine by working rat heart. Am J Physiol. 1980;239:E430-6.

130. Depre C, Ponchaut S, Deprez J, Maisin L and Hue L. Cyclic AMP suppresses the inhibition of glycolysis by alternative oxidizable substrates in the heart. J Clin Invest. 1998;101:390-7.

131. Randle PJ, Newsholme EA and Garland PB. Regulation of glucose uptake by muscle. 8 . Effects of fatty acids, ketone bodies and pyruvate, and of alloxan-diabetes and starvation, on the uptake and metabolic fate of glucose in rat heart and diaphragm muscles. Biochem J. 1964;93:652-65.

132. Meerson FZ, Spiritchev VB, Pshennikova MG and Djachkova LV. The role of the pentose-phosphate pathway in adjustment of the heart to a high load and the development of myocardial hypertrophy. Experientia. 1967;23:5302.

133. Zimmer HG, Ibel $H$ and Steinkopff $G$. Studies on the hexose monophosphate shunt in the myocardium during development of hypertrophy. Adv Myocardiol. 1980;1:487-92. 
134. Zimmer HG and Peffer $\mathrm{H}$. Metabolic aspects of the development of experimental cardiac hypertrophy. Basic Res Cardiol. 1986;81 Suppl 1:12737.

135. Gupte SA, Levine RJ, Gupte RS, Young ME, Lionetti V, Labinskyy V, Floyd BC, Ojaimi C, Bellomo M, Wolin MS and Recchia FA. Glucose-6-phosphate dehydrogenase-derived NADPH fuels superoxide production in the failing heart. J Mol Cell Cardiol. 2006;41:340-9.

136. Hecker PA, Leopold JA, Gupte SA, Recchia FA and Stanley WC. Impact of glucose-6-phosphate dehydrogenase deficiency on the pathophysiology of cardiovascular disease. Am J Physiol Heart Circ Physiol. 2013;304:H491500.

137. Jain M, Cui L, Brenner DA, Wang B, Handy DE, Leopold JA, Loscalzo J, Apstein CS and Liao R. Increased myocardial dysfunction after ischemiareperfusion in mice lacking glucose-6-phosphate dehydrogenase. Circulation. 2004;109:898-903.

138. Katare R, Caporali A, Emanueli C and Madeddu P. Benfotiamine improves functional recovery of the infarcted heart via activation of pro-survival G6PD/Akt signaling pathway and modulation of neurohormonal response. J Mol Cell Cardiol. 2010;49:625-38.

139. McCommis KS, Douglas DL, Krenz M and Baines CP. Cardiac-specific hexokinase 2 overexpression attenuates hypertrophy by increasing pentose phosphate pathway flux. J Am Heart Assoc. 2013;2:e000355. 
140. Zimmer HG and Ibel H. Ribose accelerates the repletion of the ATP pool during recovery from reversible ischemia of the rat myocardium. $J \mathrm{Mol}$ Cell Cardiol. 1984;16:863-6.

141. Zuurbier CJ, Eerbeek O, Goedhart PT, Struys EA, Verhoeven NM, Jakobs C and Ince C. Inhibition of the pentose phosphate pathway decreases ischemia-reperfusion-induced creatine kinase release in the heart. Cardiovasc Res. 2004;62:145-53.

142. Krishnan J, Suter M, Windak R, Krebs T, Felley A, Montessuit C, TokarskaSchlattner M, Aasum E, Bogdanova A, Perriard E, Perriard JC, Larsen T, Pedrazzini T and Krek W. Activation of a HIF1alpha-PPARgamma axis underlies the integration of glycolytic and lipid anabolic pathways in pathologic cardiac hypertrophy. Cell metabolism. 2009;9:512-24.

143. Blackmore PF and Shuman EA. Regulation of hepatic altro heptulose 1,7bisphosphate levels and control of flux through the pentose pathway by fructose 2,6-bisphosphate. FEBS Lett. 1982;142:255-9.

144. Boada J, Roig T, Perez X, Gamez A, Bartrons R, Cascante M and Bermudez J. Cells overexpressing fructose-2,6-bisphosphatase showed enhanced pentose phosphate pathway flux and resistance to oxidative stress. FEBS Lett. 2000;480:261-4.

145. Yamamoto T, Takano N, Ishiwata K, Ohmura M, Nagahata Y, Matsuura T, Kamata A, Sakamoto K, Nakanishi T, Kubo A, Hishiki T and Suematsu M. Reduced methylation of PFKFB3 in cancer cells shunts glucose towards the pentose phosphate pathway. Nature communications. 2014;5:3480. 
146. Yi W, Clark PM, Mason DE, Keenan MC, Hill C, Goddard WA, 3rd, Peters EC, Driggers EM and Hsieh-Wilson LC. Phosphofructokinase 1 glycosylation regulates cell growth and metabolism. Science. 2012;337:975-80.

147. Dassanayaka S and Jones SP. O-GIcNAc and the cardiovascular system. Pharmacol Ther. 2014;142:62-71.

148. Hart GW, Kreppel LK, Comer FI, Arnold CS, Snow DM, Ye Z, Cheng X, DellaManna D, Caine DS, Earles BJ, Akimoto Y, Cole RN and Hayes BK. O-GlcNAcylation of key nuclear and cytoskeletal proteins: reciprocity with O-phosphorylation and putative roles in protein multimerization. Glycobiology. 1996;6:711-6.

149. Ngoh GA, Facundo HT, Hamid T, Dillmann W, Zachara NE and Jones SP. Unique hexosaminidase reduces metabolic survival signal and sensitizes cardiac myocytes to hypoxia/reoxygenation injury. Circ Res. 2009;104:419.

150. Zachara NE and Hart GW. O-GlcNAc a sensor of cellular state: the role of nucleocytoplasmic glycosylation in modulating cellular function in response to nutrition and stress. Biochim Biophys Acta. 2004;1673:13-28.

151. Nelson BA, Robinson KA, Koning JS and Buse MG. Effects of exercise and feeding on the hexosamine biosynthetic pathway in rat skeletal muscle. Am J Physiol. 1997;272:E848-55. 
152. Medford HM, Porter K and Marsh SA. Immediate effects of a single exercise bout on protein O-GIcNAcylation and chromatin regulation of cardiac hypertrophy. Am J Physiol Heart Circ Physiol. 2013;305:H114-23.

153. Belke DD. Swim-exercised mice show a decreased level of protein OGlcNAcylation and expression of O-GIcNAc transferase in heart. J Appl Physiol (1985). 2011;111:157-62.

154. Bennett CE, Johnsen VL, Shearer $J$ and Belke DD. Exercise training mitigates aberrant cardiac protein O-GIcNAcylation in streptozotocininduced diabetic mice. Life Sci. 2013;92:657-63.

155. Roberts DJ, Tan-Sah VP, Ding EY, Smith JM and Miyamoto S. HexokinaseII positively regulates glucose starvation-induced autophagy through TORC1 inhibition. Molecular cell. 2014;53:521-33.

156. Sullivan JE, Brocklehurst KJ, Marley AE, Carey F, Carling D and Beri RK. Inhibition of lipolysis and lipogenesis in isolated rat adipocytes with AICAR, a cell-permeable activator of AMP-activated protein kinase. FEBS Lett. 1994;353:33-6.

157. Neufer PD, Bamman MM, Muoio DM, Bouchard C, Cooper DM, Goodpaster BH, Booth FW, Kohrt WM, Gerszten RE, Mattson MP, Hepple RT, Kraus WE, Reid MB, Bodine SC, Jakicic JM, Fleg JL, Williams JP, Joseph L, Evans M, Maruvada P, Rodgers M, Roary M, Boyce AT, Drugan JK, Koenig JI, Ingraham RH, Krotoski D, Garcia-Cazarin M, McGowan JA and Laughlin MR. Understanding the Cellular and Molecular Mechanisms of Physical Activity-Induced Health Benefits. Cell metabolism. 2015;22:4-11. 
158. Bernstein D. Exercise assessment of transgenic models of human cardiovascular disease. Physiol Genomics. 2003;13:217-26.

159. Niebauer J, Maxwell AJ, Lin PS, Tsao PS, Kosek J, Bernstein D and Cooke JP. Impaired aerobic capacity in hypercholesterolemic mice: partial reversal by exercise training. Am J Physiol. 1999;276:H1346-54.

160. Wang $Y$, Wisloff $U$ and Kemi OJ. Animal models in the study of exerciseinduced cardiac hypertrophy. Physiol Res. 2010;59:633-44.

161. Perrino C, Gargiulo G, Pironti G, Franzone A, Scudiero L, De Laurentis M, Magliulo F, Ilardi F, Carotenuto G, Schiattarella GG and Esposito G. Cardiovascular effects of treadmill exercise in physiological and pathological preclinical settings. Am J Physiol Heart Circ Physiol. 2011;300:H1983-9.

162. Ebihara S, Tsuji K and Kondo K. Strain differences of the mouse's freerunning circadian rhythm in continuous darkness. Physiol Behav. 1978;20:795-9.

163. Lerman I, Harrison BC, Freeman K, Hewett TE, Allen DL, Robbins J and Leinwand LA. Genetic variability in forced and voluntary endurance exercise performance in seven inbred mouse strains. J Appl Physiol (1985). 2002;92:2245-55.

164. Barbato JC, Koch LG, Darvish A, Cicila GT, Metting PJ and Britton SL. Spectrum of aerobic endurance running performance in eleven inbred strains of rats. J Appl Physiol (1985). 1998;85:530-6. 
165. Lightfoot JT, Turner MJ, Debate KA and Kleeberger SR. Interstrain variation in murine aerobic capacity. Med Sci Sports Exerc. 2001;33:2053-7.

166. Massett MP and Berk BC. Strain-dependent differences in responses to exercise training in inbred and hybrid mice. Am J Physiol Regul Integr Comp Physiol. 2005;288:R1006-13.

167. Battey J, Jordan E, Cox D and Dove W. An action plan for mouse genomics. Nat Genet. 1999;21:73-5.

168. Taketo M, Schroeder AC, Mobraaten LE, Gunning KB, Hanten G, Fox RR, Roderick TH, Stewart CL, Lilly F, Hansen CT and et al. FVB/N: an inbred mouse strain preferable for transgenic analyses. Proc Natl Acad Sci U S A. 1991;88:2065-9.

169. Kohsaka A, Laposky AD, Ramsey KM, Estrada C, Joshu C, Kobayashi Y, Turek FW and Bass J. High-fat diet disrupts behavioral and molecular circadian rhythms in mice. Cell metabolism. 2007;6:414-21.

170. Laposky AD, Bass J, Kohsaka A and Turek FW. Sleep and circadian rhythms: key components in the regulation of energy metabolism. FEBS Lett. 2008;582:142-51.

171. Arble DM, Bass J, Laposky AD, Vitaterna MH and Turek FW. Circadian timing of food intake contributes to weight gain. Obesity (Silver Spring). 2009;17:2100-2.

172. Cummins TD, Holden CR, Sansbury BE, Gibb AA, Shah J, Zafar N, Tang Y, Hellmann J, Rai SN, Spite M, Bhatnagar A and Hill BG. Metabolic 
remodeling of white adipose tissue in obesity. American journal of physiology Endocrinology and metabolism. 2014;307:E262-77.

173. Sansbury BE, Cummins TD, Tang Y, Hellmann J, Holden CR, Harbeson MA, Chen Y, Patel RP, Spite M, Bhatnagar A and Hill BG. Overexpression of Endothelial Nitric Oxide Synthase Prevents Diet-Induced Obesity and Regulates Adipocyte Phenotype. Circulation Research. 2012;111:11761189.

174. De Angelis K, Wichi RB, Jesus WR, Moreira ED, Morris M, Krieger EM and Irigoyen MC. Exercise training changes autonomic cardiovascular balance in mice. J Appl Physiol (1985). 2004;96:2174-8.

175. Dudley GA, Abraham WM and Terjung RL. Influence of exercise intensity and duration on biochemical adaptations in skeletal muscle. J Appl Physiol Respir Environ Exerc Physiol. 1982;53:844-50.

176. Hildebrandt AL, Pilegaard H and Neufer PD. Differential transcriptional activation of select metabolic genes in response to variations in exercise intensity and duration. American journal of physiology Endocrinology and metabolism. 2003;285:E1021-7.

177. McConnell TR. Practical considerations in the testing of VO2max in runners. Sports Med. 1988;5:57-68.

178. Ferreira JC, Rolim NP, Bartholomeu JB, Gobatto CA, Kokubun E and Brum PC. Maximal lactate steady state in running mice: effect of exercise training. Clin Exp Pharmacol Physiol. 2007;34:760-5. 
179. Hakimi P, Yang J, Casadesus G, Massillon D, Tolentino-Silva F, Nye CK, Cabrera ME, Hagen DR, Utter CB, Baghdy Y, Johnson DH, Wilson DL, Kirwan JP, Kalhan SC and Hanson RW. Overexpression of the cytosolic form of phosphoenolpyruvate carboxykinase (GTP) in skeletal muscle repatterns energy metabolism in the mouse. J Biol Chem. 2007;282:3284455.

180. Pederson BA, Cope CR, Schroeder JM, Smith MW, Irimia JM, Thurberg BL, DePaoli-Roach AA and Roach PJ. Exercise capacity of mice genetically lacking muscle glycogen synthase: in mice, muscle glycogen is not essential for exercise. J Biol Chem. 2005;280:17260-5.

181. Billat VL, Mouisel E, Roblot $\mathrm{N}$ and Melki J. Inter- and intrastrain variation in mouse critical running speed. J Appl Physiol (1985). 2005;98:1258-63.

182. Von Wittke $\mathrm{P}$, Lindner A, Deegen $\mathrm{E}$ and Sommer H. Effects of training on blood lactate-running speed relationship in thoroughbred racehorses. $J$ Appl Physiol (1985). 1994;77:298-302.

183. Gladden LB. Lactate metabolism: a new paradigm for the third millennium. The Journal of physiology. 2004;558:5-30.

184. Salabei JK, Lorkiewicz PK, Mehra P, Gibb AA, Haberzettl P, Hong KU, Wei X, Zhang X, Li Q, Wysoczynski M, Bolli R, Bhatnagar A and Hill BG. Type 2 Diabetes Dysregulates Glucose Metabolism in Cardiac Progenitor Cells. J Biol Chem. 2016;291:13634-48.

185. Verwey M, Robinson B and Amir S. Recording and analysis of circadian rhythms in running-wheel activity in rodents. J Vis Exp. 2013. 
186. Kadaja L, Eimre M, Paju K, Roosimaa M, Podramagi T, Kaasik P, Pehme A, Orlova E, Mudist M, Peet N, Piirsoo A, Seene T, Gellerich FN and Seppet EK. Impaired oxidative phosphorylation in overtrained rat myocardium. Exp Clin Cardiol. 2010;15:e116-27.

187. Moskowitz AS, Terman GW and Liebeskind JC. Stress-induced analgesia in the mouse: strain comparisons. Pain. 1985;23:67-72.

188. Pavone F, Castellano C and Oliverio A. Strain-dependent effects of shockinduced release of opioids: dissociation between analgesia and behavioral seizures. Brain Res. 1986;366:326-8.

189. Stavnes K and Sprott RL. Effects of age and genotype on acquisition of an active avoidance response in mice. Dev Psychobiol. 1975;8:437-45.

190. Moraska A, Deak T, Spencer RL, Roth D and Fleshner M. Treadmill running produces both positive and negative physiological adaptations in SpragueDawley rats. Am J Physiol Regul Integr Comp Physiol. 2000;279:R1321-9.

191. Mori T and Makino J. [Response types to shock and avoidance learning in inbred strains of mice]. Shinrigaku Kenkyu. 1994;65:295-302.

192. Pugh PL, Ahmed SF, Smith MI, Upton N and Hunter AJ. A behavioural characterisation of the FVB/N mouse strain. Behav Brain Res. 2004;155:283-9.

193. Henry KR and Chole RA. Genotypic differences in behavioral, physiological and anatomical expressions of age-related hearing loss in the laboratory mouse. Audiology. 1980;19:369-83. 
194. Mikaelian DO. Development and degeneration of hearing in the C57/b16 mouse: relation of electrophysiologic responses from the round window and cochlear nucleus to cochlear anatomy and behavioral responses. Laryngoscope. 1979;89:1-15.

195. Willott JF. Effects of aging, hearing loss, and anatomical location on thresholds of inferior colliculus neurons in C57BL/6 and CBA mice. $J$ Neurophysiol. 1986;56:391-408.

196. Katzeff HL, Bovbjerg D and Mark DA. Exercise regulation of triiodothyronine metabolism. Am J Physiol. 1988;255:E824-8.

197. Carter GT, Wineinger MA, Walsh SA, Horasek SJ, Abresch RT and Fowler WM, Jr. Effect of voluntary wheel-running exercise on muscles of the $\mathrm{mdx}$ mouse. Neuromuscul Disord. 1995;5:323-32.

198. Konhilas JP, Chen H, Luczak E, McKee LA, Regan J, Watson PA, Stauffer BL, Khalpey ZI, McKinsey TA, Horn T, LaFleur B and Leinwand LA. Diet and sex modify exercise and cardiac adaptation in the mouse. Am J Physiol Heart Circ Physiol. 2015;308:H135-45.

199. Werner C, Hanhoun M, Widmann T, Kazakov A, Semenov A, Poss J, Bauersachs J, Thum T, Pfreundschuh M, Muller P, Haendeler J, Bohm M and Laufs $U$. Effects of physical exercise on myocardial telomere-regulating proteins, survival pathways, and apoptosis. J Am Coll Cardiol. 2008;52:47082. 
200. Falls WA, Fox JH and MacAulay CM. Voluntary exercise improves both learning and consolidation of cued conditioned fear in C57 mice. Behav Brain Res. 2010;207:321-31.

201. Werner C, Furster T, Widmann T, Poss J, Roggia C, Hanhoun M, Scharhag J, Buchner N, Meyer T, Kindermann W, Haendeler J, Bohm M and Laufs U. Physical exercise prevents cellular senescence in circulating leukocytes and in the vessel wall. Circulation. 2009;120:2438-47.

202. Konhilas JP, Widegren U, Allen DL, Paul AC, Cleary A and Leinwand LA. Loaded wheel running and muscle adaptation in the mouse. Am J Physiol Heart Circ Physiol. 2005;289:H455-65.

203. Kemi OJ, Loennechen JP, Wisloff $U$ and Ellingsen O. Intensity-controlled treadmill running in mice: cardiac and skeletal muscle hypertrophy. J Appl Physiol (1985). 2002;93:1301-9.

204. Ferreira JC, Bacurau AV, Bueno CR, Jr., Cunha TC, Tanaka LY, Jardim MA, Ramires PR and Brum PC. Aerobic exercise training improves Ca2+ handling and redox status of skeletal muscle in mice. Exp Biol Med (Maywood). 2010;235:497-505.

205. Sturgeon K, Muthukumaran G, Ding D, Bajulaiye A, Ferrari V and Libonati JR. Moderate-intensity treadmill exercise training decreases murine cardiomyocyte cross-sectional area. Physiol Rep. 2015;3.

206. Ericsson M, Andersson KB, Amundsen BH, Torp SH, Sjaastad I, Christensen G, Sejersted OM and Ellingsen O. High-intensity exercise 
training in mice with cardiomyocyte-specific disruption of Serca2. J Appl Physiol (1985). 2010;108:1311-20.

207. Miyagi MY, Seelaender M, Castoldi A, de Almeida DC, Bacurau AV, Andrade-Oliveira V, Enjiu LM, Pisciottano M, Hayashida CY, Hiyane MI, Brum PC, Camara NO and Amano MT. Long-term aerobic exercise protects against cisplatin-induced nephrotoxicity by modulating the expression of IL6 and HO-1. PLoS One. 2014;9:e108543.

208. Narkar VA, Downes M, Yu RT, Embler E, Wang YX, Banayo E, Mihaylova MM, Nelson MC, Zou Y, Juguilon H, Kang H, Shaw RJ and Evans RM. AMPK and PPARdelta agonists are exercise mimetics. Cell. 2008;134:40515.

209. Petrosino JM, Heiss VJ, Maurya SK, Kalyanasundaram A, Periasamy M, LaFountain RA, Wilson JM, Simonetti OP and Ziouzenkova O. Graded Maximal Exercise Testing to Assess Mouse Cardio-Metabolic Phenotypes. PLoS One. 2016;11:e0148010.

210. Wisloff U, Helgerud J, Kemi OJ and Ellingsen O. Intensity-controlled treadmill running in rats: $\mathrm{VO}(2$ max $)$ and cardiac hypertrophy. Am J Physiol Heart Circ Physiol. 2001;280:H1301-10.

211. Vega RB, Konhilas JP, Kelly DP and Leinwand LA. Molecular Mechanisms Underlying Cardiac Adaptation to Exercise. Cell metabolism. 2017;25:10121026.

212. Lopez-Otin C, Galluzzi L, Freije JM, Madeo F and Kroemer G. Metabolic Control of Longevity. Cell. 2016;166:802-21. 
213. Bassett DR, Jr. and Howley ET. Limiting factors for maximum oxygen uptake and determinants of endurance performance. Med Sci Sports Exerc. 2000;32:70-84.

214. Wilson MG, Ellison GM and Cable NT. Basic science behind the cardiovascular benefits of exercise. Br J Sports Med. 2016;50:93-9.

215. Roh J, Rhee J, Chaudhari V and Rosenzweig A. The Role of Exercise in Cardiac Aging: From Physiology to Molecular Mechanisms. Circ Res. 2016;118:279-95.

216. Seals DR. Edward F. Adolph Distinguished Lecture: The remarkable antiaging effects of aerobic exercise on systemic arteries. J Appl Physiol (1985). 2014;117:425-39.

217. Mann $\mathrm{N}$ and Rosenzweig A. Can exercise teach us how to treat heart disease? Circulation. 2012;126:2625-35.

218. Lerchenmuller C and Rosenzweig A. Mechanisms of exercise-induced cardiac growth. Drug Discov Today. 2014;19:1003-9.

219. Ryall KA, Bezzerides VJ, Rosenzweig A and Saucerman JJ. Phenotypic screen quantifying differential regulation of cardiac myocyte hypertrophy identifies CITED4 regulation of myocyte elongation. J Mol Cell Cardiol. 2014;72:74-84.

220. Donthi RV, Ye G, Wu C, McClain DA, Lange AJ and Epstein PN. Cardiac expression of kinase-deficient 6-phosphofructo-2-kinase/fructose-2,6bisphosphatase inhibits glycolysis, promotes hypertrophy, impairs myocyte function, and reduces insulin sensitivity. J Biol Chem. 2004;279:48085-90. 
221. Wang Q, Donthi RV, Wang J, Lange AJ, Watson LJ, Jones SP and Epstein PN. Cardiac phosphatase-deficient 6-phosphofructo-2-kinase/fructose-2,6bisphosphatase increases glycolysis, hypertrophy, and myocyte resistance to hypoxia. Am J Physiol Heart Circ Physiol. 2008;294:H2889-97.

222. Sansbury BE, DeMartino AM, Xie Z, Brooks AC, Brainard RE, Watson LJ, DeFilippis AP, Cummins TD, Harbeson MA, Brittian KR, Prabhu SD, Bhatnagar A, Jones SP and Hill BG. Metabolomic analysis of pressureoverloaded and infarcted mouse hearts. Circulation Heart failure. 2014;7:634-42.

223. Watson LJ, Facundo HT, Ngoh GA, Ameen M, Brainard RE, Lemma KM, Long BW, Prabhu SD, Xuan YT and Jones SP. O-linked beta-Nacetylglucosamine transferase is indispensable in the failing heart. Proc Natl Acad Sci U S A. 2010;107:17797-802.

224. Ashcroft SJ, Weerasinghe LC, Bassett JM and Randle PJ. The pentose cycle and insulin release in mouse pancreatic islets. The Biochemical journal. 1972;126:525-32.

225. Sato S, Ogura Y, Mishra V, Shin J, Bhatnagar S, Hill BG and Kumar A. TWEAK promotes exercise intolerance by decreasing skeletal muscle oxidative phosphorylation capacity. Skeletal muscle. 2013;3:18.

226. Xia J, Sinelnikov IV, Han B and Wishart DS. MetaboAnalyst 3.0--making metabolomics more meaningful. Nucleic acids research. 2015;43:W251-7. 
227. Benjamini $Y$ and Hochberg $Y$. Controlling the false discovery rate: a practical and powerful approach to multiple testing. Journal of the Royal Statistical Society Series. 1995;B57.

228. Gautier L, Cope L, Bolstad BM and Irizarry RA. affy--analysis of Affymetrix GeneChip data at the probe level. Bioinformatics (Oxford, England). 2004;20:307-15.

229. Ritchie ME, Phipson B, Wu D, Hu Y, Law CW, Shi W and Smyth GK. limma powers differential expression analyses for RNA-sequencing and microarray studies. Nucleic acids research. 2015;43:e47.

230. Huang da W, Sherman BT and Lempicki RA. Systematic and integrative analysis of large gene lists using DAVID bioinformatics resources. Nat Protoc. 2009;4:44-57.

231. Bourajjaj M, Armand AS, da Costa Martins PA, Weijts B, van der Nagel R, Heeneman S, Wehrens $\mathrm{XH}$ and De Windt LJ. NFATc2 is a necessary mediator of calcineurin-dependent cardiac hypertrophy and heart failure. $J$ Biol Chem. 2008;283:22295-303.

232. Brown GC. Control of respiration and ATP synthesis in mammalian mitochondria and cells. The Biochemical journal. 1992;284 ( Pt 1):1-13.

233. Mor I, Cheung EC and Vousden $\mathrm{KH}$. Control of glycolysis through regulation of PFK1: old friends and recent additions. Cold Spring Harb Symp Quant Biol. 2011;76:211-6. 
234. Yalcin A, Telang S, Clem B and Chesney J. Regulation of glucose metabolism by 6-phosphofructo-2-kinase/fructose-2,6-bisphosphatases in cancer. Experimental and molecular pathology. 2009;86:174-9.

235. Cortassa S, Caceres V, Bell LN, O'Rourke B, Paolocci N and Aon MA. From metabolomics to fluxomics: a computational procedure to translate metabolite profiles into metabolic fluxes. Biophys J. 2015;108:163-72.

236. Deprez J, Vertommen D, Alessi DR, Hue L and Rider MH. Phosphorylation and activation of heart 6-phosphofructo-2-kinase by protein kinase $B$ and other protein kinases of the insulin signaling cascades. J Biol Chem. 1997;272:17269-75.

237. Pozuelo Rubio M, Peggie M, Wong BH, Morrice N and MacKintosh C. 143-3s regulate fructose-2,6-bisphosphate levels by binding to PKBphosphorylated cardiac fructose-2,6-bisphosphate kinase/phosphatase. EMBO J. 2003;22:3514-23.

238. Alessi DR, Andjelkovic M, Caudwell B, Cron P, Morrice N, Cohen P and Hemmings BA. Mechanism of activation of protein kinase B by insulin and IGF-1. EMBO J. 1996;15:6541-51.

239. Hue $L$ and Taegtmeyer $H$. The Randle cycle revisited: a new head for an old hat. American journal of physiology Endocrinology and metabolism. 2009;297:E578-91.

240. Depre C, Rider MH, Veitch K and Hue L. Role of fructose 2,6-bisphosphate in the control of heart glycolysis. J Biol Chem. 1993;268:13274-9. 
241. Fillmore N, Mori J and Lopaschuk GD. Mitochondrial fatty acid oxidation alterations in heart failure, ischaemic heart disease and diabetic cardiomyopathy. Br J Pharmacol. 2014;171:2080-90.

242. Wang J, Xu J, Wang Q, Brainard RE, Watson LJ, Jones SP and Epstein PN. Reduced cardiac fructose 2,6 bisphosphate increases hypertrophy and decreases glycolysis following aortic constriction. PLOS One. 2013;8:e53951.

243. Lai L, Leone TC, Keller MP, Martin OJ, Broman AT, Nigro J, Kapoor K, Koves TR, Stevens R, Ilkayeva OR, Vega RB, Attie AD, Muoio DM and Kelly DP. Energy Metabolic Re-Programming in the Hypertrophied and Early Stage Failing Heart: A Multi-systems Approach. Circulation Heart failure. 2014.

244. Tews B, Roerig P, Hartmann C, Hahn M, Felsberg J, Blaschke B, Sabel M, Kunitz A, Toedt G, Neben K, Benner A, von Deimling A, Reifenberger G and Lichter P. Hypermethylation and transcriptional downregulation of the CITED4 gene at 1 p34.2 in oligodendroglial tumours with allelic losses on $1 p$ and 19q. Oncogene. 2007;26:5010-6.

245. Noor E, Eden E, Milo R and Alon U. Central carbon metabolism as a minimal biochemical walk between precursors for biomass and energy. Molecular cell. 2010;39:809-20.

246. Laczy B, Hill BG, Wang K, Paterson AJ, White CR, Xing D, Chen YF, Darley-Usmar V, Oparil S and Chatham JC. Protein O-GIcNAcylation: a new 
signaling paradigm for the cardiovascular system. Am J Physiol Heart Circ Physiol. 2009;296:H13-28.

247. Lunde IG, Aronsen JM, Kvaloy H, Qvigstad E, Sjaastad I, Tonnessen T, Christensen G, Gronning-Wang LM and Carlson CR. Cardiac O-GIcNAc signaling is increased in hypertrophy and heart failure. Physiol Genomics. 2012;44:162-72.

248. Facundo HT, Brainard RE, Watson LJ, Ngoh GA, Hamid T, Prabhu SD and Jones SP. O-GlcNAc signaling is essential for NFAT-mediated transcriptional reprogramming during cardiomyocyte hypertrophy. Am J Physiol Heart Circ Physiol. 2012;302:H2122-30.

249. Dassanayaka S, Brainard RE, Watson LJ, Long BW, Brittian KR, DeMartino AM, Aird AL, Gumpert AM, Audam TN, Kilfoil PJ, Muthusamy S, Hamid T, Prabhu SD and Jones SP. Cardiomyocyte Ogt limits ventricular dysfunction in mice following pressure overload without affecting hypertrophy. Basic Res Cardiol. 2017;112:23.

250. Bouche C, Serdy S, Kahn CR and Goldfine AB. The cellular fate of glucose and its relevance in type 2 diabetes. Endocr Rev. 2004;25:807-30.

251. Brownlee M. Biochemistry and molecular cell biology of diabetic complications. Nature. 2001;414:813-20.

252. Sage AT, Walter LA, Shi Y, Khan MI, Kaneto H, Capretta A and Werstuck $\mathrm{GH}$. Hexosamine biosynthesis pathway flux promotes endoplasmic reticulum stress, lipid accumulation, and inflammatory gene expression in 
hepatic cells. American journal of physiology Endocrinology and metabolism. 2010;298:E499-511.

253. James LR, Tang D, Ingram A, Ly H, Thai K, Cai L and Scholey JW. Flux through the hexosamine pathway is a determinant of nuclear factor kappaBdependent promoter activation. Diabetes. 2002;51:1146-56.

254. Zimmer HG. The oxidative pentose phosphate pathway in the heart: regulation, physiological significance, and clinical implications. Basic Res Cardiol. 1992;87:303-16.

255. Palm DC, Rohwer JM and Hofmeyr JH. Regulation of glycogen synthase from mammalian skeletal muscle--a unifying view of allosteric and covalent regulation. FEBS J. 2013;280:2-27.

256. Coleman RA and Lee DP. Enzymes of triacylglycerol synthesis and their regulation. Prog Lipid Res. 2004;43:134-76.

257. Kalhan SC and Hanson RW. Resurgence of serine: an often neglected but indispensable amino Acid. J Biol Chem. 2012;287:19786-91.

258. Grant GA. Contrasting catalytic and allosteric mechanisms for phosphoglycerate dehydrogenases. Arch Biochem Biophys. 2012;519:17585.

259. Opie LH, Mansford KR and Owen P. Effects of increased heart work on glycolysis and adenine nucleotides in the perfused heart of normal and diabetic rats. The Biochemical journal. 1971;124:475-90. 
260. Neely JR, Denton RM, England PJ and Randle PJ. The effects of increased heart work on the tricarboxylate cycle and its interactions with glycolysis in the perfused rat heart. The Biochemical journal. 1972;128:147-59.

261. Clark MG and Patten GS. Epinephrine activation of phosphofructokinase in perfused rat heart independent of changes in effector concentrations. J Biol Chem. 1981;256:27-30.

262. Nascimben L, Ingwall JS, Lorell BH, Pinz I, Schultz V, Tornheim K and Tian R. Mechanisms for increased glycolysis in the hypertrophied rat heart. Hypertension. 2004;44:662-7.

263. Van Schaftingen E, Jett MF, Hue L and Hers HG. Control of liver 6phosphofructokinase by fructose 2,6-bisphosphate and other effectors. Proc Natl Acad Sci U S A. 1981;78:3483-6.

264. van Berlo JH, Maillet $M$ and Molkentin JD. Signaling effectors underlying pathologic growth and remodeling of the heart. J Clin Invest. 2013;123:3745.

265. Almeida A, Moncada S and Bolanos JP. Nitric oxide switches on glycolysis through the AMP protein kinase and 6-phosphofructo-2-kinase pathway. Nat Cell Biol. 2004;6:45-51.

266. Novellasdemunt L, Tato I, Navarro-Sabate A, Ruiz-Meana M, MendezLucas A, Perales JC, Garcia-Dorado D, Ventura F, Bartrons R and Rosa JL. Akt-dependent activation of the heart 6-phosphofructo-2kinase/fructose-2,6-bisphosphatase (PFKFB2) isoenzyme by amino acids. J Biol Chem. 2013;288:10640-51. 
267. Bockus LB and Humphries KM. cAMP-dependent Protein Kinase (PKA) Signaling Is Impaired in the Diabetic Heart. J Biol Chem. 2015;290:292508.

268. Jones SP, Zachara NE, Ngoh GA, Hill BG, Teshima Y, Bhatnagar A, Hart GW and Marban E. Cardioprotection by $\mathrm{N}$-acetylglucosamine linkage to cellular proteins. Circulation. 2008;117:1172-82.

269. Sansbury BE, Riggs DW, Brainard RE, Salabei JK, Jones SP and Hill BG. Responses of hypertrophied myocytes to reactive species: implications for glycolysis and electrophile metabolism. Biochemical Journal. 2011;435:519-528.

270. Darville MI, Crepin KM, Hue L and Rousseau GG. 5' flanking sequence and structure of a gene encoding rat 6-phosphofructo-2-kinase/fructose-2,6bisphosphatase. Proc Natl Acad Sci U S A. 1989;86:6543-7.

271. Kurland IJ, el-Maghrabi MR, Correia JJ and Pilkis SJ. Rat liver 6phosphofructo-2-kinase/fructose-2,6-bisphosphatase. Properties of phospho- and dephospho- forms and of two mutants in which Ser32 has been changed by site-directed mutagenesis. J Biol Chem. 1992;267:441623.

272. Vertommen D, Bertrand L, Sontag B, Di Pietro A, Louckx MP, Vidal H, Hue $\mathrm{L}$ and Rider MH. The ATP-binding site in the 2-kinase domain of liver 6phosphofructo-2-kinase/fructose-2,6-bisphosphatase. Study of the role of Lys-54 and Thr-55 by site-directed mutagenesis. J Biol Chem. 1996;271:17875-80. 
273. Radde BN, Ivanova MM, Mai HX, Salabei JK, Hill BG and Klinge CM. Bioenergetic differences between MCF-7 and T47D breast cancer cells and their regulation by oestradiol and tamoxifen. The Biochemical journal. 2015;465:49-61.

274. Salabei JK, Lorkiewicz PK, Holden CR, Li Q, Hong KU, Bolli R, Bhatnagar A and Hill BG. Glutamine Regulates Cardiac Progenitor Cell Metabolism and Proliferation. Stem cells. 2015;33:2613-27.

275. Lane AN, Fan TW, Xie Z, Moseley HN and Higashi RM. Isotopomer analysis of lipid biosynthesis by high resolution mass spectrometry and NMR. Analytica chimica acta. 2009;651:201-8.

276. Le A, Lane AN, Hamaker M, Bose S, Gouw A, Barbi J, Tsukamoto T, Rojas CJ, Slusher BS, Zhang H, Zimmerman LJ, Liebler DC, Slebos RJ, Lorkiewicz PK, Higashi RM, Fan TW and Dang CV. Glucose-independent glutamine metabolism via TCA cycling for proliferation and survival in B cells. Cell metabolism. 2012;15:110-21.

277. Lorkiewicz P, Higashi RM, Lane AN and Fan TW. High information throughput analysis of nucleotides and their isotopically enriched isotopologues by direct-infusion FTICR-MS. Metabolomics. 2012;8:930939.

278. Wei X, Lorkiewicz PK, Shi B, Salabei JK, Hill BG, Kim S, McClain CJ and Zhang X. Analysis of stable isotope assisted metabolomics data acquired by high resolution mass spectrometry. Analytical Methods. 2017;9:22752283. 
279. Hill BG, Dranka BP, Zou L, Chatham JC and Darley-Usmar VM. Importance of the bioenergetic reserve capacity in response to cardiomyocyte stress induced by 4-hydroxynonenal. The Biochemical journal. 2009;424:99-107.

280. Lane AN and Fan TWM. Regulation of mammalian nucleotide metabolism and biosynthesis. Nucleic acids research. 2015;43:2466-2485.

281. Sellers K, Fox MP, Bousamra M, 2nd, Slone SP, Higashi RM, Miller DM, Wang Y, Yan J, Yuneva MO, Deshpande R, Lane AN and Fan TW. Pyruvate carboxylase is critical for non-small-cell lung cancer proliferation. J Clin Invest. 2015;125:687-98.

282. Kim TT and Dyck JR. The role of CD36 in the regulation of myocardial lipid metabolism. Biochim Biophys Acta. 2016;1860:1450-60.

283. Tamboli A, Vander Maten M, O'Looney P and Vahouny GV. Metabolism of fatty acid, glycerol and a monoglyceride analogue by rat cardiac myocytes and perfused hearts. Lipids. 1983;18:808-13.

284. Tamboli A, O'Looney P, Vander Maten M and Vahouny GV. Comparative metabolism of free and esterified fatty acids by the perfused rat heart and rat cardiac myocytes. Biochim Biophys Acta. 1983;750:404-10.

285. Owen OE, Kalhan SC and Hanson RW. The key role of anaplerosis and cataplerosis for citric acid cycle function. J Biol Chem. 2002;277:30409-12.

286. Crabtree HG. Observations on the carbohydrate metabolism of tumours. The Biochemical journal. 1929;23:536-45. 
287. Diaz-Ruiz R, Rigoulet M and Devin A. The Warburg and Crabtree effects: On the origin of cancer cell energy metabolism and of yeast glucose repression. Biochim Biophys Acta. 2011;1807:568-76.

288. Sansbury BE, Jones SP, Riggs DW, Darley-Usmar VM and Hill BG. Bioenergetic function in cardiovascular cells: the importance of the reserve capacity and its biological regulation. Chemico-biological interactions. 2011;191:288-95.

289. Salabei JK, Lorkiewicz PK, Holden CR, Li Q, Hong KU, Bolli R, Bhatnagar A and Hill BG. Glutamine regulates cardiac progenitor cell metabolism and proliferation. Stem Cells. 2015;33:2613-2627.

290. Mookerjee SA, Goncalves RL, Gerencser AA, Nicholls DG and Brand MD. The contributions of respiration and glycolysis to extracellular acid production. Biochim Biophys Acta. 2015;1847:171-81.

291. Mookerjee SA, Gerencser AA, Nicholls DG and Brand MD. Quantifying intracellular rates of glycolytic and oxidative ATP production and consumption using extracellular flux measurements. J Biol Chem. 2017;292:7189-7207.

292. Taegtmeyer H, Lam $\mathrm{T}$ and Davogustto G. Cardiac Metabolism in Perspective. Compr Physiol. 2016;6:1675-1699.

293. Marshall S, Bacote V and Traxinger RR. Discovery of a metabolic pathway mediating glucose-induced desensitization of the glucose transport system. Role of hexosamine biosynthesis in the induction of insulin resistance. J Biol Chem. 1991;266:4706-12. 
294. Goodwin GW, Cohen DM and Taegtmeyer H. [5-3H]glucose overestimates glycolytic flux in isolated working rat heart: role of the pentose phosphate pathway. American journal of physiology Endocrinology and metabolism. 2001;280:E502-8.

295. Funato $\mathrm{Y}$, Hayashi T, Irino $\mathrm{Y}$, Takenawa $\mathrm{T}$ and Miki $\mathrm{H}$. Nucleoredoxin regulates glucose metabolism via phosphofructokinase 1. Biochem Biophys Res Commun. 2013;440:737-42.

296. Franklin DA, He Y, Leslie PL, Tikunov AP, Fenger N, Macdonald JM and Zhang Y. p53 coordinates DNA repair with nucleotide synthesis by suppressing PFKFB3 expression and promoting the pentose phosphate pathway. Sci Rep. 2016;6:38067.

297. Miyazawa H, Yamaguchi Y, Sugiura Y, Honda K, Kondo K, Matsuda F, Yamamoto T, Suematsu M and Miura M. Rewiring of embryonic glucose metabolism via suppression of PFK-1 and aldolase during mouse chorioallantoic branching. Development. 2017;144:63-73.

298. Rodriguez-Rodriguez P, Fernandez E, Almeida A and Bolanos JP. Excitotoxic stimulus stabilizes PFKFB3 causing pentose-phosphate pathway to glycolysis switch and neurodegeneration. Cell Death Differ. 2012;19:1582-9.

299. Lehninger AL, Nelson DL and Cox MM. Lehninger principles of biochemistry. 3rd ed. New York: Worth Publishers; 2000.

300. Moseley HN, Lane AN, Belshoff AC, Higashi RM and Fan TW. A novel deconvolution method for modeling UDP-N-acetyl-D-glucosamine 
biosynthetic pathways based on (13)C mass isotopologue profiles under non-steady-state conditions. BMC Biol. 2011;9:37.

301. Frenzel J, Schellenberger W, Eschrich K and Hofmann E. Control of the fructose 6-phosphate/fructose 2,6-bisphosphate cycle by sn-glycerol 3phosphate. Biomed Biochim Acta. 1988;47:461-70.

302. Huo Y, Guo X, Li H, Wang H, Zhang W, Wang Y, Zhou H, Gao Z, Telang S, Chesney J, Chen YE, Ye J, Chapkin RS and Wu C. Disruption of inducible 6-phosphofructo-2-kinase ameliorates diet-induced adiposity but exacerbates systemic insulin resistance and adipose tissue inflammatory response. J Biol Chem. 2010;285:3713-21.

303. Huo Y, Guo X, Li H, Xu H, Halim V, Zhang W, Wang H, Fan YY, Ong KT, Woo SL, Chapkin RS, Mashek DG, Chen Y, Dong H, Lu F, Wei L and Wu C. Targeted overexpression of inducible 6-phosphofructo-2-kinase in adipose tissue increases fat deposition but protects against diet-induced insulin resistance and inflammatory responses. I Biol Chem. 2012;287:21492-500.

304. Gibb AA, Epstein PN, Uchida S, Zheng Y, McNally LA, Obal D, Katragadda K, Trainor PJ, Conklin DJ, Brittian KR, Tseng MT, Wang J, Jones SP, Bhatnagar A and Hill BG. Exercise-Induced Changes in Glucose Metabolism Promote Physiologic Cardiac Growth. Circulation. 2017.

305. Gibb AA, Lorkiewicz PK, Zheng YT, Zhang X, Bhatnagar A, Jones SP and Hill BG. Integration of flux measurements to resolve changes in anabolic 
and catabolic metabolism in cardiac myocytes. The Biochemical journal. $2017 ; 474: 2785-2801$.

306. Koliadko NG. [Activity of enzymes of the oxidative part of the pentose phosphate pathway in skeletal muscles during muscular activity of different types and during the recovery period]. Ukr Biokhim Zh (1978). 1981;53:10610.

307. Summermatter S, Baum O, Santos G, Hoppeler H and Handschin C. Peroxisome proliferator-activated receptor \{gamma\} coactivator 1 alpha\} (PGC-1\{alpha\}) promotes skeletal muscle lipid refueling in vivo by activating de novo lipogenesis and the pentose phosphate pathway. $J$ Biol Chem. 2010;285:32793-800.

308. Liang MT, Glonek T, Meneses P, Kopp SJ, Paulson DJ, Gierke LW and Schwartz FN. NMR spectroscopy study of heart phospholipids. An exercise and anabolic steroids effect. Int J Sports Med. 1992;13:417-23.

309. Lim HY, Wang W, Wessells RJ, Ocorr K and Bodmer R. Phospholipid homeostasis regulates lipid metabolism and cardiac function through SREBP signaling in Drosophila. Genes Dev. 2011;25:189-200.

310. Tappia PS and Singal T. Phospholipid-mediated signaling and heart disease. Subcell Biochem. 2008;49:299-324.

311. Lin CS, Sun YL and Liu CY. Structural and biochemical evidence of mitochondrial depletion in pigs with hypertrophic cardiomyopathy. Res Vet Sci. 2003;74:219-26. 
312. Bugger H, Schwarzer M, Chen D, Schrepper A, Amorim PA, Schoepe M, Nguyen TD, Mohr FW, Khalimonchuk O, Weimer BC and Doenst T. Proteomic remodelling of mitochondrial oxidative pathways in pressure overload-induced heart failure. Cardiovasc Res. 2010;85:376-84.

313. Bugger $\mathrm{H}$ and Abel ED. Mitochondria in the diabetic heart. Cardiovasc Res. 2010;88:229-40.

314. Murray AJ, Cole MA, Lygate CA, Carr CA, Stuckey DJ, Little SE, Neubauer $\mathrm{S}$ and Clarke K. Increased mitochondrial uncoupling proteins, respiratory uncoupling and decreased efficiency in the chronically infarcted rat heart. $J$ Mol Cell Cardiol. 2008;44:694-700.

315. Hatch GM. Regulation of cardiolipin biosynthesis in the heart. Mol Cell Biochem. 1996;159:139-48.

316. Houtkooper RH and Vaz FM. Cardiolipin, the heart of mitochondrial metabolism. Cell Mol Life Sci. 2008;65:2493-506.

317. Paradies G, Petrosillo G, Pistolese M and Ruggiero FM. Reactive oxygen species generated by the mitochondrial respiratory chain affect the complex III activity via cardiolipin peroxidation in beef-heart submitochondrial particles. Mitochondrion. 2001;1:151-9.

318. Saini-Chohan HK, Holmes MG, Chicco AJ, Taylor WA, Moore RL, McCune SA, Hickson-Bick DL, Hatch GM and Sparagna GC. Cardiolipin biosynthesis and remodeling enzymes are altered during development of heart failure. J Lipid Res. 2009;50:1600-8. 
319. Sparagna GC, Chicco AJ, Murphy RC, Bristow MR, Johnson CA, Rees ML, Maxey ML, McCune SA and Moore RL. Loss of cardiac tetralinoleoyl cardiolipin in human and experimental heart failure. J Lipid Res. 2007;48:1559-70.

320. Mentch SJ and Locasale JW. One-carbon metabolism and epigenetics: understanding the specificity. Ann N Y Acad Sci. 2016;1363:91-8.

321. Shiraki N, Shiraki Y, Tsuyama T, Obata F, Miura M, Nagae G, Aburatani H, Kume K, Endo $\mathrm{F}$ and Kume $\mathrm{S}$. Methionine metabolism regulates maintenance and differentiation of human pluripotent stem cells. Cell metabolism. 2014;19:780-94.

322. Tang X, Keenan MM, Wu J, Lin CA, Dubois L, Thompson JW, Freedland SJ, Murphy SK and Chi JT. Comprehensive profiling of amino acid response uncovers unique methionine-deprived response dependent on intact creatine biosynthesis. PLoS Genet. 2015;11:e1005158.

323. Ulanovskaya OA, Zuhl AM and Cravatt BF. NNMT promotes epigenetic remodeling in cancer by creating a metabolic methylation sink. Nat Chem Biol. 2013;9:300-6.

324. Sidhu S, Gangasani A, Korotchkina LG, Suzuki G, Fallavollita JA, Canty JM, Jr. and Patel MS. Tissue-specific pyruvate dehydrogenase complex deficiency causes cardiac hypertrophy and sudden death of weaned male mice. Am J Physiol Heart Circ Physiol. 2008;295:H946-H952.

325. Arad M, Maron BJ, Gorham JM, Johnson WH, Jr., Saul JP, Perez-Atayde AR, Spirito P, Wright GB, Kanter RJ, Seidman CE and Seidman JG. 
Glycogen storage diseases presenting as hypertrophic cardiomyopathy. The New England journal of medicine. 2005;352:362-72.

326. Schannwell CM, Zimmermann T, Schneppenheim M, Plehn G, Marx R and Strauer BE. Left ventricular hypertrophy and diastolic dysfunction in healthy pregnant women. Cardiology. 2002;97:73-8.

327. Gehart H, Kumpf S, Ittner A and Ricci R. MAPK signalling in cellular metabolism: stress or wellness? EMBO Rep. 2010;11:834-40.

328. Grassian AR, Metallo CM, Coloff JL, Stephanopoulos G and Brugge JS. Erk regulation of pyruvate dehydrogenase flux through PDK4 modulates cell proliferation. Genes Dev. 2011;25:1716-33.

329. Whiteman EL, Cho $\mathrm{H}$ and Birnbaum MJ. Role of Akt/protein kinase $\mathrm{B}$ in metabolism. Trends Endocrinol Metab. 2002;13:444-51.

330. Liao H, Winkfein RJ, Mack G, Rattner JB and Yen TJ. CENP-F is a protein of the nuclear matrix that assembles onto kinetochores at late $\mathrm{G} 2$ and is rapidly degraded after mitosis. J Cell Biol. 1995;130:507-18. 


\section{APPENDIX}

The contents of Chapters 2, 3, and 4 are derived from published manuscripts. Copyright clearance from the publishers has been granted and the manuscripts have been properly cited by the inclusion of footnotes and proper citations within the reference section of this dissertation. 


\title{
CURRICULUM VITAE
}

Andrew A. Gibb, M.S. 2733 Field Ave Apt 1

Louisville, KY 40206

Phone: 216-409-1353 (cell); 502-852-1029 (work)

Email: Andrew.gibb@louisville.edu

\section{EDUCATION}

06/2009 B.S. in Exercise Physiology, Ohio University, Athens, OH

05/2011 M.S. in Exercise Physiology, University of Louisville, Louisville, KY

12/2015 M.S. in Physiology, University of Louisville, Louisville, KY

12/2017 Ph.D. in Physiology, University of Louisville, Louisville, KY

\section{ACADEMIC APPOINTMENTS}

\author{
08/2009 - 05/2011 Graduate Teaching Assistant \\ Department of Health and Sport Science \\ Exercise Physiology Program \\ University of Louisville \\ Louisville, KY \\ 08/2011 - 12/2012 Adjunct Instructor \\ Department of Health and Sport Science \\ Exercise Physiology Program \\ University of Louisville \\ Louisville, KY \\ 08/2012 - present Doctoral Candidate \\ Department of Physiology \\ University of Louisville \\ Louisville, KY
}

\section{OTHER POSITION AND EMPLOYMENT}

01/2010 - 09/2011 Clinical Exercise Physiologist - Kentucky One Healthcare, Cardiac Rehabilitation, Louisville, KY 
09/2011 - 08/2012 Fitness Coordinator - Corporate Fitness Works, Humana Corporate Headquarters, Louisville KY

02/2016 - present Research Associate - Department of Medicine, University of Louisville, Louisville, KY

\section{PROFESSIONAL DEVELOPMENT}

Nov 2014

Isotope Tracers in Metabolic Research: Principles and practices of kinetic analysis; Mouse Metabolic Phenotyping Center (MMPC) Consortium, Training

Conference/Workshop. Nov. 10-14, Cleveland, OH.

\section{PROFESSIONAL MEMBERSHIPS AND ACTIVITIES}

$\begin{array}{ll}2008-\text { present } & \begin{array}{l}\text { American College of Sports Medicine (ACSM), Student } \\ \text { Member }\end{array} \\ 2014-\text { present } & \text { American Heart Association (AHA), Student Member } \\ 2015-\text { present } & \text { American Physiological Society (APS), Student Member } \\ 2016-\text { present } & \text { American Association for the Advancement of Science }\end{array}$
(AAAS).

\section{HONORS AND AWARDS}

$2005-2009$
$2009-2011$
$2012-2014$
2016

2016
2016 - present
2016 - 2019

2017

\section{SERVICE}

$2015-2017$
Gateway Scholarship, Ohio University

Graduate Assistantship, Department of Exercise Physiology, University of Louisville Integrated Programs in Biomedical Sciences (IPIBS) Fellowship Award, University of Louisville NIKE, Inc. Loren G. Myhre Environmental and Exercise Physiology Predoctoral Research Award, EEP Section of the American Physiological Society (APS) Physiology Departmental Graduate Student Travel Award American Heart Association Predoctoral Fellowship Award Nominated for a 3-year sponsored membership to AAAS by the associate dean of graduate and postdoctoral studies, University of Louisville

Abstract Travel Award, APS Physiological Bioenergetics Conference

School of Medicine Graduate Council Student Representative 


\section{PUBLICATIONS}

\section{Peer-Reviewed Publications}

1. Salabei JK, Gibb AA, Hill BG. Comprehensive measurement of respiratory activity in permeabilized cells using extracellular flux analysis. Nat Protoc 9(2):421-38, 2014.

2. Cummins TD, Holden CR, Sansbury BE, Gibb AA, Shah J, Zafar N, Tang Y, Hellmann J, Rai SN, Spite M, Bhatnagar A, Hill BG. Metabolic remodeling of white adipose tissue in obesity. Am J Physiol Endocrinol Metab 307(3):E26277, 2014.

3. Salabei JK, Lorkiewicz PK, Mehra P, Gibb AA, Haberzettl P, Hong KU, Wei X, Zhang X, Li Q, Wysoczynski M, Bolli R, Bhatnagar A, Hill BG. Type 2 diabetes dysregulates glucose metabolism in cardiac progenitor cells. J Biol Chem 291:13634-48, 2016.

4. Gibb AA, Gilbert LA, Riggs DW, Conklin DJ, Bhatnagar A, Hill BG. FVB/NJ mice are a useful model for examining cardiometabolic adaptations to treadmill exercise. Front Physiol 7:636, 2016.

5. Gibb AA, Lorkiewicz PK, Zheng YT, Zhang X, Bhatnagar A, Jones SP, Hill BG. Integration of flux measurements to resolve changes in anabolic and catabolic metabolism in cardiac myocytes. Biochem J, 474: 2785-2801, 2017.

6. Gibb AA, Epstein PN, Uchida S, Zheng YT, McNally LA, Obal D, Katragadda $\mathrm{K}$, Trainor P, Conklin DJ, Brittian KR, Tseng MT, Wang J, Jones SP, Bhatnagar A, Hill BG. Exercise-induced changes in glucose metabolism promote physiologic cardiac growth. Circulation, 2017. (In Press)

\section{Manuscripts in Review}

1. Osuma E, Riggs D, Gibb AA, Hill BG. High throughput measurement of metabolism in planarians reveals activation of glycolysis during regeneration. Regeneration, 2017.

2. Liu M, Ding C, Luo F, Albeituni S, Hu X, Hamada M, Takahashi S, Gibb AA, Kloecker G, Zhang H, Buosamra II M, Fan TW, Lane A, Hill BG, Yan J. Transcription factor c-Maf controls anti-tumor immunity by programming immunosuppressive macrophages in lung cancer. Sci Transl Med, 2017. 


\section{Manuscripts in Preparation}

1. Gibb AA \& Hill BG. Metabolic regulation of myocardial remodeling. (Invited Review, Circ Res)

2. Pena Claderin E, McNally L, Gibb AA, Hill BG, Hellmann J. A diet high in fat inhibits exercise-induced biosynthesis of pro-resolving lipid mediators. Manuscript in preparation.

\section{INVITED TALKS}

1. Center for Metabolic Origins of Disease Sanford Burnham Prebys Medical Discovery Institute

Title: Metabolic regulation of cardiac adaptation to exercise.

Host: Doug Lewandowski, Ph.D. April, 2017.

2. Center for Translational Medicine

Lewis Katz School of Medicine, Temple University

Title: Metabolic regulation of cardiac adaptation to exercise.

Host: John Elrod, PhD. August, 2017.

3. Abstract Selection for Oral Presentation

Session Title: Symposium - Energy School II

Title: Phosphofructokinase coordinates mitochondrial and ancillary

biosynthetic pathway activities in the cardiomyocyte

APS Bioenergetics, August, 2017

\section{ABSTRACTS}

1. Salabei JK, Gibb AA, Jones SP, Bhatnagar A, Hill BG. Regulation of the hyperproliferative vascular smooth muscle cell phenotype by mitochondrial fission. Free Radic Biol Med 65: S2, 2013.

2. Holden CR, Wysoczynski M, Sansbury B, Hellmann J, Zafar N, Gibb A, Bhatnagar A, and Hill BG. Nutrient excess promotes accumulation of bone marrow-derived progenitor cells in adipose tissue. FASEB J 28(S1): 641.12, 2014.

3. Gibb AA, Sansbury BE, DeMartino AM, Harbeson MA, Jones SP, Bhatnagar A, Hill BG. Regulation of glucose metabolism in the failing heart. $2^{\text {nd }}$ Annual Cardiovascular Forum for Promoting Centers of Excellence and Young Investigators, 2014. 
4. Gibb AA, Sansbury BE, DeMartino AM, Harbeson MA, Jones SP, Bhatnagar A, Hill BG. Regulation of glucose metabolism in the failing heart. Research Louisville, 2014.

5. Gibb AA, Bhatnagar A, Hill BG. Role of cardiac glycolysis in adaptations to exercise. Circ Res 117: A387, 2015.

6. Gibb AA, Bhatnagar A, Hill BG. Myocardial glycolytic rate regulates exercise capacity and cardiac hypertrophy. Southeast Regional IDeA Conference, 2015.

7. Gibb AA, Gilbert LA, Brittian KR, Jones SP, Bhatnagar A, Hill BG. Myocardial glycolytic rate regulates exercise-induced physiologic cardiac growth. FASEB J 30(S1):1239.7, 2016.

8. Gibb AA, Gilbert LA, Lorkiewicz P, Trainor P, Tseng MT, Epstein PN, Bhatnagar A, Hill BG. Altered cardiomyocyte glycolysis causes cardiac hypertrophy and mitochondrial dysfunction. Circulation 134:A19448, 2016.

9. Gibb AA, Lorkiewicz PK, Zheng YT, Zhang X, Bhatnagar A, Jones SP, Hill BG. Phosphofructokinase coordinates mitochondrial and ancillary biosynthetic pathway activities in the cardiomyocyte. ABP Bioenergetics, 2017.

10. Gibb AA, Epstein PN, Uchida S, Zheng YT, McNally LA, Trainor P, Conklin DJ, Brittian KR, Tseng MT, Jones SP, Bhatnagar A, Hill BG. Metabolic regulation of exercise-induced cardiac growth. APS Bioenergetics, 2017.

11.Zheng YT, Gibb AA, McNally LA, Liu S, Merchant M, Hill BG. Multimeric metabolic enzyme complexes in the adult mammalian heart. APS Bioenergetics, 2017.

\section{GRANTS AND CONTRACTS}

16PRE31010022

Gibb (PI)

American Heart Association Predoctoral Fellowship, Great Rives Affiliate Title: Metabolic Regulation of Myocardial Adaptations to Exercise

The goal of this project is to understand the role of metabolism, specifically glucose metabolism, in the adaptive responses associated with exercise training. \$52,000, 2016-2018.

Priority Score: 1.42; Application Percentile Rank: 0.33\%.

Intramural Grant

College of Education Internal Funding Award (University of Louisville)

Equipment procurement for teaching aid technology (CleveMed Software)

Spring 2011

Direct Costs: $\$ 5,000$ - Role: Lead 


\section{INVITED JOURNAL REVIEWER}

1. Toxicological Sciences (1 Review)

\section{ASSISTED JOURNAL REVIEWER}

1. Circulation Research (2 Reviews)

2. The American Journal of Physiology - Heart and Circulatory Physiology (2 Reviews)

\section{ACADEMIC INSTRUCTION}

Fall 2009

Spring 2010

Summer 2010

Fall 2010

Spring 2011

Fall 2011

Fall 2012
Instructor, University of Louisville, Louisville KY

Weight Training HSS 109

Fitness Walking HSS 114

Instructor, University of Louisville, Louisville KY Indoor Soccer HSS 150

Instructor, University of Louisville, Louisville KY

Outdoor Soccer HSS 136

Weight Training HSS 109

Instructor, University of Louisville, Louisville KY

Physical Fitness and Conditioning HSS 110

Fitness Walking HSS 114

Volleyball HSS 137

Instructor, University of Louisville, Louisville KY

Weight Training HSS 109-06

Weight Training HSS 109-07

Physical Fitness and Conditioning HSS 110-01

Physical Fitness and Conditioning HSS 110-02

Adjunct Instructor, University of Louisville, Louisville KY

Principles in Electrocardiography EXP 611

Mean course evaluation - 4.63/5.0

Adjunct Instructor, University of Louisville, Louisville KY

Principles in Electrocardiography EXP 611

Mean course evaluation - 4.88/5.0

${ }^{* * *}$ Highest semester mean within the department 